Florida International University

FIU Digital Commons

FIU Electronic Theses and Dissertations

University Graduate School

$11-8-2018$

\title{
HIV Tat and Morphine-induced Neurodegeneration in a Beclin 1 Hemizygous Mouse Model
}

Jessica A. Lapierre

Florida International University, jlapi008@fiu.edu

Follow this and additional works at: https://digitalcommons.fiu.edu/etd

Part of the Immune System Diseases Commons, Molecular and Cellular Neuroscience Commons, and the Other Immunology and Infectious Disease Commons

\section{Recommended Citation}

Lapierre, Jessica A., "HIV Tat and Morphine-induced Neurodegeneration in a Beclin 1 Hemizygous Mouse Model" (2018). FIU Electronic Theses and Dissertations. 3888.

https://digitalcommons.fiu.edu/etd/3888

This work is brought to you for free and open access by the University Graduate School at FIU Digital Commons. It has been accepted for inclusion in FIU Electronic Theses and Dissertations by an authorized administrator of FIU Digital Commons. For more information, please contact dcc@fiu.edu. 


\title{
FLORIDA INTERNATIONAL UNIVERSITY \\ Miami, Florida
}

\section{HIV TAT AND MORPHINE-INDUCED NEURODEGENERATION IN A BECLIN 1 HEMIZYGOUS MOUSE MODEL}

\author{
A dissertation submitted in partial fulfillment of \\ the requirements for the degree of \\ DOCTOR OF PHILOSOPHY \\ in \\ BIOMEDICAL SCIENCES \\ by \\ Jessica Audrey Lapierre
}

2018 


\section{To: Dean John Rock \\ College of Medicine}

This dissertation, written by Jessica Audrey Lapierre, and entitled HIV Tat and Morphineinduced Neurodegeneration in a Beclin 1 Hemizygous Mouse Model, having been approved in respect to style and intellectual content, is referred to you for judgment.

We have read this dissertation and recommend that it be approved.

Alexander Agoulnik

Jeremy Chambers

Tomás Guilarte

Michal Toborek

Nazira El-Hage, Major Professor

Date of Defense: November 82018

The dissertation of Jessica Audrey Lapierre is approved.

Dean John Rock

College of Medicine

Andrés G. Gil

Vice President for Research and Economic Development and Dean of the University Graduate School

Florida International University, 2018 


\section{DEDICATION}

This work is firstly dedicated to my late grandmother Audira Armand, whose dreams far exceeded those of my own, and secondly, to future generations of minority women in STEM, may you always find your inner BGM 


\section{ACKNOWLEDGMENTS}

If you have great people around you, they will take you higher than your dreams will. Those closest to you determine your level of success. Where to even begin? This dissertation would not have been possible without several people to which I am eternally grateful. First and foremost, I don't think there are enough words to express my level of gratitude towards my supervisor and mentor Dr. Nazira El-Hage. The guidance and support you've provided have been indispensable and I can't thank you enough for the opportunities, independence, and growth you've allowed me. I would also like to thank the members of my dissertation committee, Dr. Alexander Agoulnik, Dr. Jeremy Chambers, Dr. Tomás Guilarte, and Dr. Michal Toborek for their generous participation and patience. I would especially like to thank Dr. Chambers for always having an open door and ear to provide additional guidance. I would like to express gratitude to the National Institute on Drug Abuse for my funding through the NIDA Diversity Supplement Program (R01-DA036154S1).

I was incredibly lucky to be able to work in an environment of scientific collaboration within the Immunology department and for that I extend great thanks. Special thank you to Dr. Andrea Raymond and Dr. Ajeet Kaushik for your support. Additionally, I must highlight my second family, the members of the El-Hage lab: Dr. Myosotys Rodriguez, Dr. Mohan Kumar Muthu Karuppan, Mr. Chet Raj Ojha, and Mr. Hary Estrada-Bueno. None of this work would have been possible without you and I am incredibly thankful to have had the opportunity to work with all of you. Thank you for making the day to day struggles of PhD life a little bit easier. Dr. Rodriguez, you have been a mentor, a friend, a travel buddy, a therapist, and so much more; this experience wouldn't have been the same without you. 
A great many thanks are due to the wonderful friends I made in this program, Dr. Monica Rodriguez Silva (de Jozic), Mr. Kyle Martins, and Ms. Sneham Tiwari who understood the struggle and kept me sane over the years, always quick to offer advice, friendship, and so much laughter. To my friend Mr. Kyle Ohree who was so supportive it was almost irritating, thank you. Thank you to my best friends of $12+$ years, my day 1's, Dr. Ramone Eldemire, Ms. Diana Etienne, and Ms. Myrlande Denis, all of whom I could never imagine my life without and never want to. Your friendship has been a constant source of support, happiness, comfort, and just the right amount of silly.

I would be nothing without the love and support of my family who have always lifted me up and believed in me, even when I may not have believed in myself. To my aunts, uncles, and cousins that are always there for me. To my cousin/best friend/sister Tatyana Rameau, your confidence in me is astounding. To my brothers Robert (regards) and Patrick Lapierre, thank you for inspiring me, taking care of me, accepting my eccentricities, and claiming me in public. I love you both always. Lastly, to my superwoman mom Carole Lapierre whose love, strength, and sacrifice made me the person I am today.

Nan tout kè $m$ ', di ou mèsi 


\title{
ABSTRACT OF THE DISSERTATION
}

\section{HIV TAT AND MORPHINE-INDUCED NEURODEGENERATION IN A BECLIN 1}

\author{
HEMIZYGOUS MOUSE MODEL
}

by

Jessica Audrey Lapierre

Florida International University, 2018

Miami, Florida

\section{Professor Nazira El-Hage, Major Professor}

Early in infection, HIV crosses the blood-brain barrier and induces neuropathology. Viral presence in the CNS coupled with secretion of neurotoxic proteins causes neuroinflammation, glial dysfunction, excitotoxicity, and neuronal death. Despite advances in combined antiretroviral therapy, HIV-infected patients present with a spectrum of cognitive and psychomotor deficits collectively referred to as HIV-associated neurological disorders (HAND). A subset of HAND patients abuses drugs such as opiates like heroin and morphine show an exacerbation and rapid progression of HIV neuropathology; however, the mechanisms of this synergy are not well understood. Autophagy is a lysosomal degradative process which eliminates and recycles cytosolic components and is implicated in facilitating HIV-1 replication in the CNS and periphery, and in Tat-induced neurodegeneration. When a key initiator of autophagy Beclin 1 was silenced using siRNAs, there was a marked reduction of HIV-1 replication in human microglia and astrocytes and the corresponding inflammatory response. As such, the goal of the current study is to determine if diminished Beclin 1 is neuroprotective against Tat-and morphineinduced neurodegeneration using heterozygous Beclin $1\left(\right.$ Becn $\left.^{+/-}\right)$mice. Examination of Tat-and morphine-induced inflammatory molecule secretion revealed that Becn1+/- mixed astrocyte and microglia (glia) exhibited attenuated secretion of cytokine IL-6 and 
chemokines RANTES and MCP-1 compared to control (C57BL/6J) glia, an effect mediated through the $\mu$-opioid receptor. Dysregulation of autophagy-related gene expression and excessive intracellular calcium accumulation were limited in Becn1 ${ }^{+/-}$glia. When determining the effects of Tat-and morphine co-exposure on neuronal survival in vitro, we found Becn $1^{+/-}$neurons were particularly sensitive to injury, excitotoxicity, and toxic exposures; however, when C57BL/6J neurons were exposed to conditioned media of C57BL/6J and Becn1 ${ }^{+/}$glia treated with Tat and morphine, neurons treated with Becn $1^{+/}$supernatant had better outcomes than those treated with $\mathrm{C} 57 \mathrm{BL} / 6 \mathrm{~J}$ conditioned media. Furthermore, despite minimal difference between strains in locomotor assessment, we observed significantly greater striatal neuron losses in adult C57BL/6J mice exposed to intrastriatal Tat-and systemic morphine compared to Becn $1^{+/-}$mice. Our studies demonstrate the potential of targeting Beclin 1 in glia for the prevention of Tat and opiateinduced CNS dysfunction. 


\section{TABLE OF CONTENTS}

CHAPTER 1: AN INTRODUCTION TO HIV-ASSOCIATED NEUROLOGICAL DISORDERS (HAND) AND AUTOPHAGY IN THE CONTEXT OF OPIATE ABUSE ...... 1

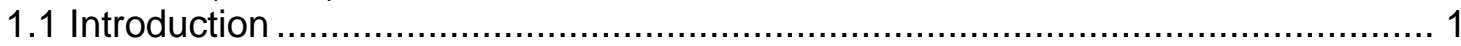

1.2 Brief overview of the HIV genome .......................................................... 4

1.3 HIV-Associated Neurological Disorders (HAND) ……................................... 8

1.3.1 Microglial activation contributions to HAND …………............................. 9

1.3.2 Astrocytic dysfunction in HAND .........................................................10

1.3.3 Cumulative effect of HIV infection on neurons ........................................10

1.4 Interlinked epidemics between HIV and opiate drug abuse .............................12

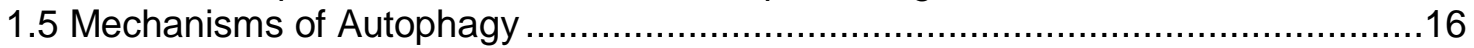

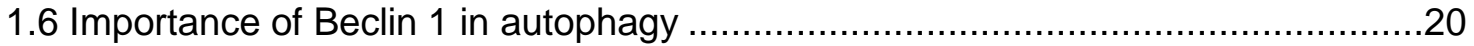

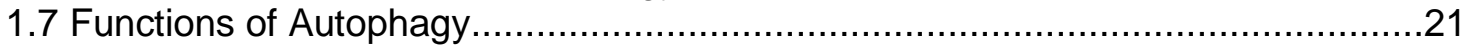

1.7.1 Autophagy and basal metabolism and homeostasis .................................21

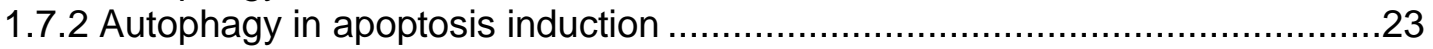

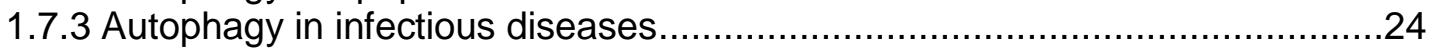

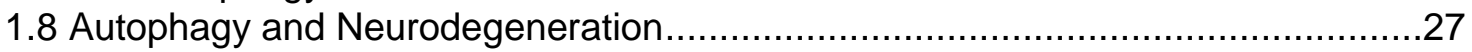

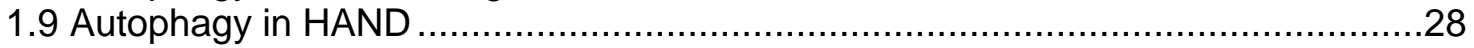

1.10 Interplay between HAND, opiates, and autophagy ........................................30

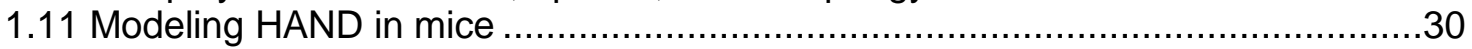

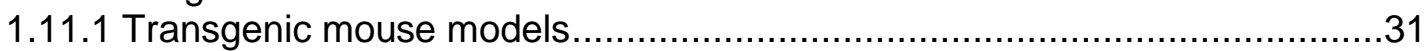

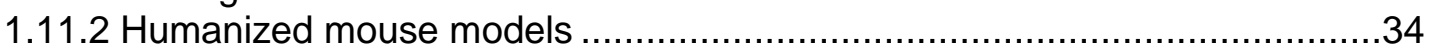

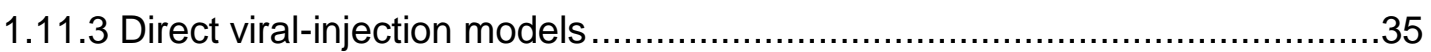

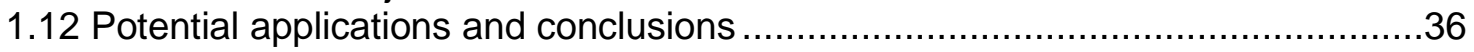

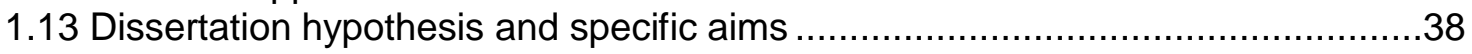

CHAPTER 2: CRITICAL ROLE OF BECLIN 1 IN HIV TAT AND MORPHINE-INDUCED GLIAL DYSFUNCTION …….......................................4

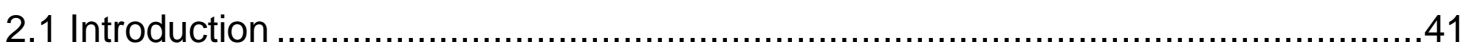

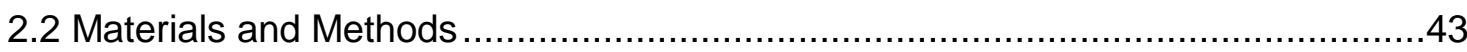

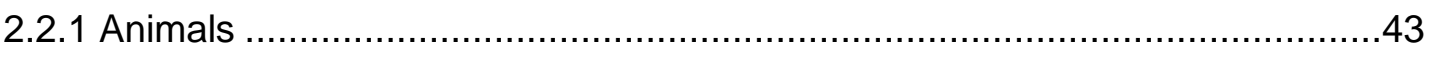

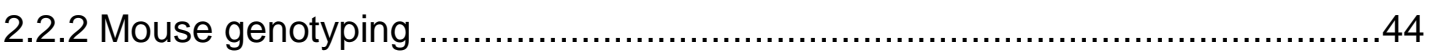

2.2.3 Mixed glial murine cell cultures ............................................................44

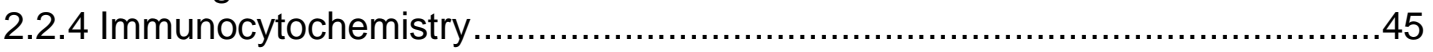

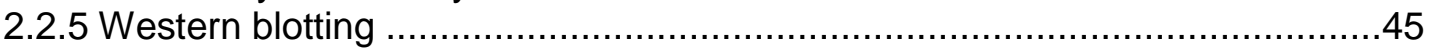

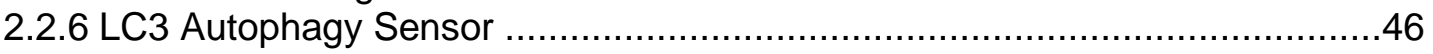

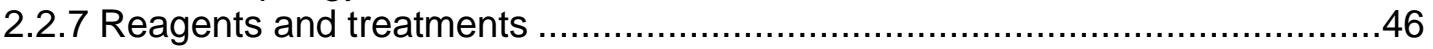

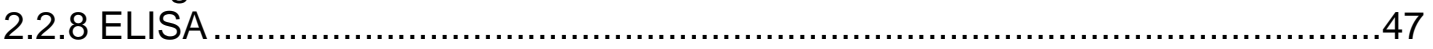

2.2.9 Quantitative Reverse Transcription-Polymerase Chain Reaction...................47

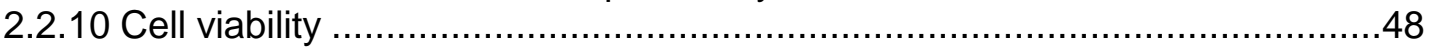

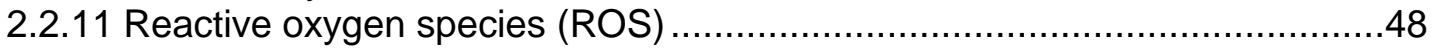

2.2.12 Nitric oxide (NO) production .............................................................49

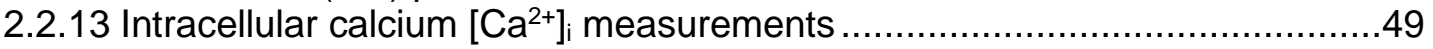

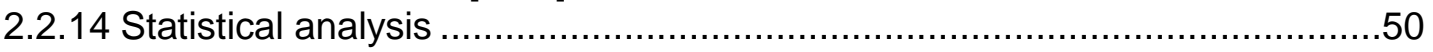

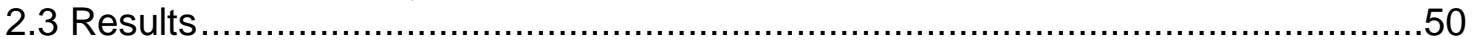

2.3.1 Molecular characterization of Beclin 1 using primary glial cell cultures 
derived from $\mathrm{C} 57 \mathrm{BL} / 6 \mathrm{~J}$ and autophagy deficient mouse.

2.3.2 Beclin $1^{+/-}$glia show reduced inflammatory molecule induction upon

exposure to the viral protein Tat....

2.3.3 Beclin 1 mediates glial inflammation induced by Tat alone and in

combination with morphine.

2.3.4 Tat and morphine use Beclin 1 to alter the autophagy pathway .....................64

2.3.5 Tat and morphine induced effects on intracellular calcium are mediated

by Beclin 1 .

2.3.6 Limited Tat and morphine-interactive effects on oxidative stress are not mediated by Beclin 1 .

2.4 Discussion.

CHAPTER 3: BECLIN 1 IS A REGULATOR OF NEURONAL OUTCOME AND KEY COMPONENT IN MEDIATING NEURO-GLIAL INTERACTIONS UPON TAT AND MORPHINE EXPOSURE

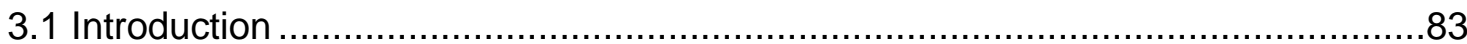

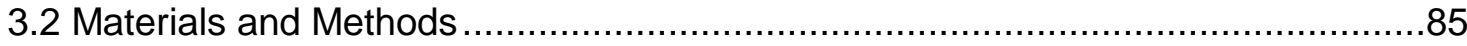

3.2.1 Timed-pregnancy and primary murine neuron cell cultures.........................85

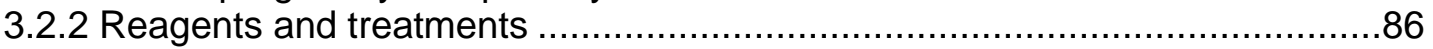

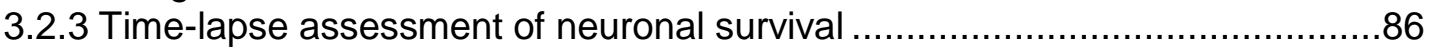

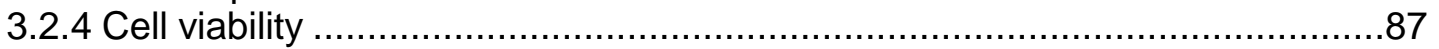

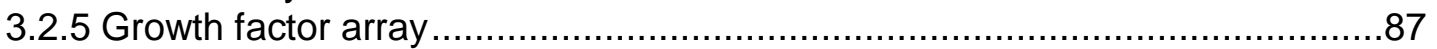

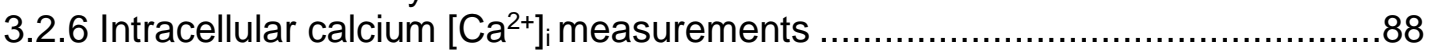

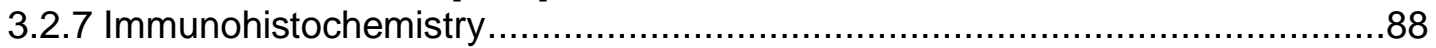

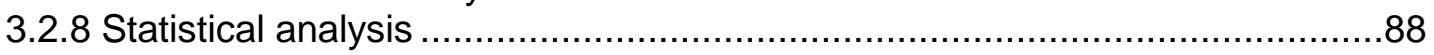

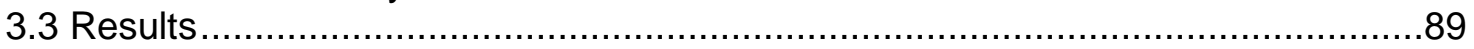

3.3.1 Neuronal Beclin 1 deficiency contributes to aberrant Tat-induced intracellular calcium accumulation and dendritic injury ..................................... 89

3.3.2 Becn $1^{+/-}$derived neurons show reduced viability with Tat and morphine exposure .........................................................................................94

3.3.3 Reduction of Beclin 1 expression in glia treated with Tat and morphine

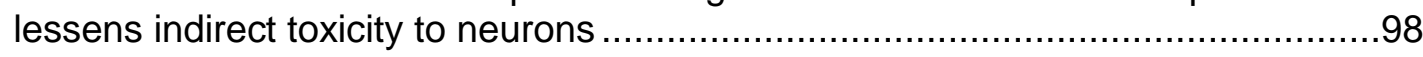

3.3.4 Beclin 1 associated alterations in growth factor secretion upon Tat and

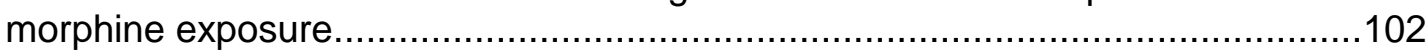

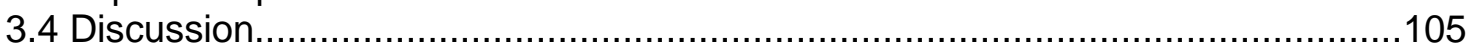

CHAPTER 4: CONTRIBUTIONS OF BECLIN 1 TO TAT AND OPIOID NEUROPATHOLOGY AND MOTOR IMPAIRMENT WITHIN THE MOUSE

STRIATUM.

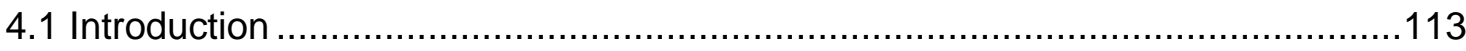

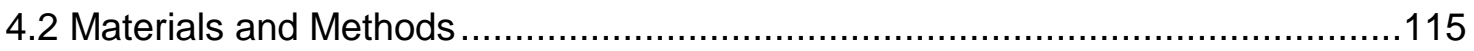

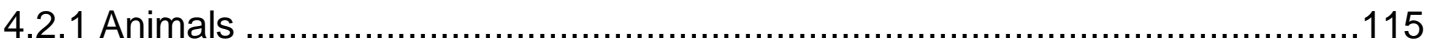

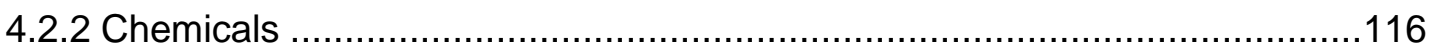

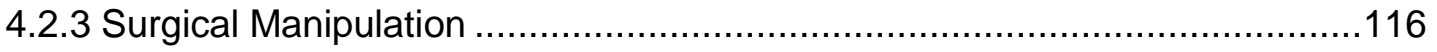

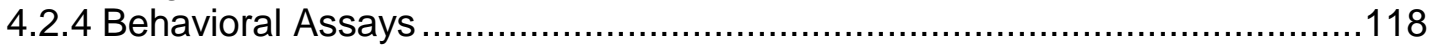

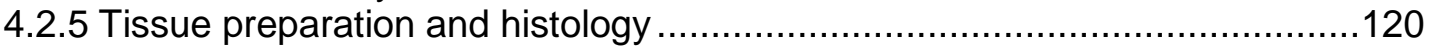

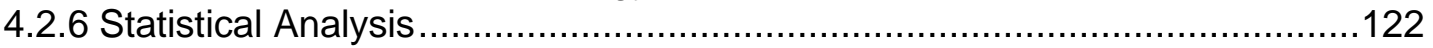

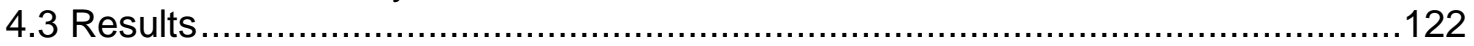

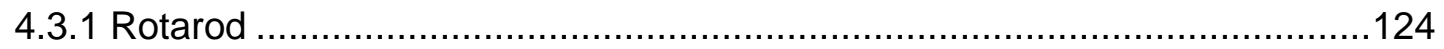




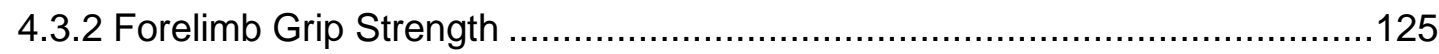

4.3.3 Horizontal Bars .................................................................................... 126

4.3.4 Striatal neuron losses caused by Tat and morphine co-exposure ................128

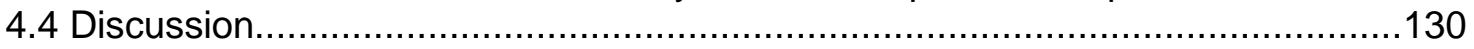

CHAPTER 5: FINAL CONCLUSIONS AND FUTURE DIRECTIONS .........................137

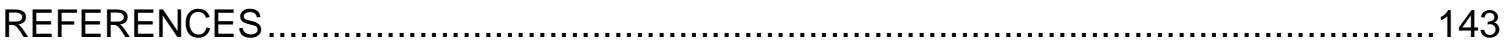

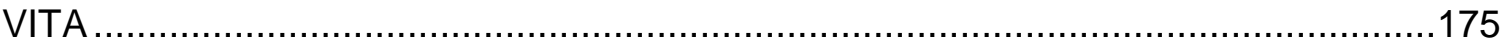




\section{LIST OF FIGURES}

FIGURE

PAGE

Figure 1.1 Summary of mammalian autophagy pathways...................................... 3

Figure 1.2 Diagram depicting components of HIV virion and the viral genome .............. 5

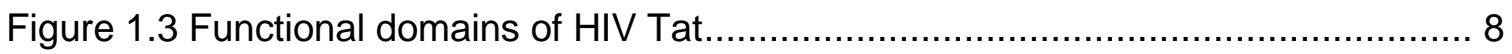

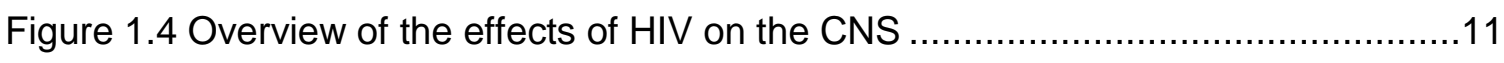

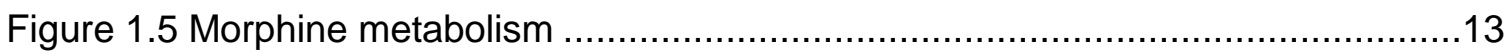

Figure 1.6 Mechanisms of opioid receptor signaling .........................................

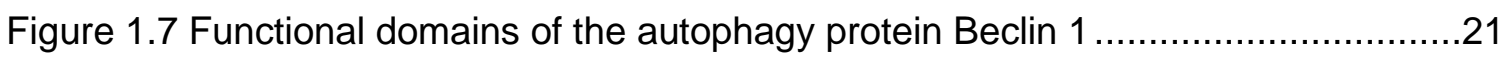

Figure 1.8 Various mouse models which can be used to study autophagy in

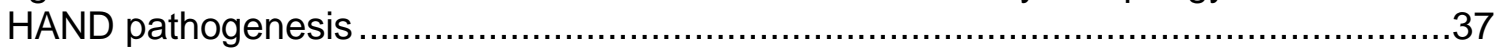

Figure 2.1 Characterization of Beclin 1-deficient mixed glia ..................................51

Figure 2.2 Autophagy induction in C57BL/6J and Becn1 ${ }^{+/}$glia ..................................52

Figure 2.3 Immunocytochemistry for glial cell presence identifying GFAP ${ }^{+}$

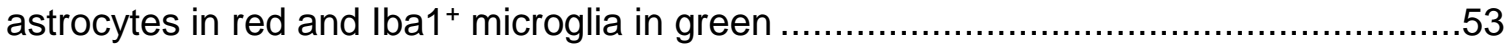

Figure 2.4 Beclin 1 facilitates Tat-induced inflammatory molecule secretion.................55

Figure 2.5 Inactive Tat is unable to interact with Beclin1 to induce cytokine secretion ...56

Figure 2.6 Effects of viral proteins on inflammatory molecule secretion through

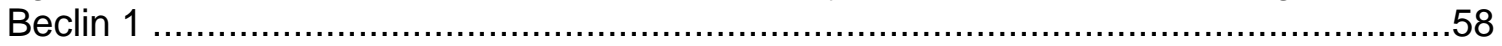

Figure 2.7 Tat induction of autophagy dysregulation...............................................59

Figure 2.8 Tat and morphine induced inflammatory effects show contribution of Beclin 1

Figure 2.9 Differential effects on opioid receptor mRNA upon Tat and morphine exposure

Figure 2.10 Glial toxicity of Tat and morphine. 64

Figure 2.11 Beclin 1-mediated autophagy dysregulation by Tat and morphine 66

Figure 2.12 Tat and morphine enhanced intracellular calcium release is prevented by reduction of Beclin 1 expression. 
Figure 2.13 Beclin 1 is independent of oxidative stress induced by Tat with limited contribution of morphine.

Figure 2.14 Schematic diagram summarizing the differential effects of Tat and morphine on the autophagy pathway in C57BL/6J and Beclin 1-deficient mouse glia....81

Figure 3.1 Primary culture and imaging of C57BL/6J and Becn1 ${ }^{+/-}$neurons. 89

Figure 3.2 Becn1+/- neurons exhibit increased intracellular calcium accumulation upon Tat exposure.

Figure 3.3 Tat exposure to Becn1 ${ }^{+/-}$neurons induces elevated dendritic beading .94

Figure 3.4 Neurotoxic effects of Tat and morphine are more severe in Becn $1^{+/}$ neurons

Figure 3.5 Decreased toxicity of neurons exposed to supernatant from Becn $1^{\text {+/- }}$ glia treated with Tat and morphine.

Figure 3.6 Tat and morphine treatment in Becn1+/- glia increases secretion of neurotrophic growth factors 105

Figure 4.1 Experimental design and surgical plan of Tat and morphine exposure .......116

Figure 4.2 Stereotaxic coordinates and surgical example.....

Figure 4.3 Representative images of motor skill behavioral assessments .118

Figure 4.4 Post-fixed brain tissue and schematic representation of image sampling for histological assessment

Figure 4.5 Mortality of mice following intracranial injection and subcutaneous pellet implantation

Figure 4.6 Tat exposure causes no detectable impairment of rotarod performance in C57BL/6J or Becn1 ${ }^{+/-}$mice.

Figure 4.7 Tat and morphine exposure show delayed effects to grip strength.

Figure 4.8 Becn1 $1^{+-}$mice outperform C57BL/6J exposed to Tat but not co-treated with morphine in horizontal bars

Figure 4.9 Tat and morphine co-exposure causes less neuronal loss in the Becn $1^{+/}$striatum 


\section{ABBREVIATIONS AND ACRONYMS}

Alzheimer's disease $\quad$ AD

$\begin{array}{lll}\text { Acquired immunodeficiency syndrome } & \text { AIDS }\end{array}$

Adenosine monophosphate-activated protein kinase AMPK

$\begin{array}{ll}\text { Autophagy-related genes } & \text { ATG }\end{array}$

BCL-2 associated athanogene $3 \quad$ BAG3

$\begin{array}{ll}\text { Blood-brain barrier } & \text { BBB }\end{array}$

Combined antiretroviral therapy $\quad$ cART

$\begin{array}{ll}\text { Central nervous system } & \text { CNS }\end{array}$

$\begin{array}{ll}\text { Delta }(\delta) \text {-opioid receptor } & \text { DOR }\end{array}$

Glial fibrillary acidic protein $\quad$ GFAP

HIV-associated neurological disorders HAND

Human immunodeficiency virus HIV

HIV-encephalitis $\quad$ HIVE

$\begin{array}{ll}\text { Injection drug users } & \text { IDU }\end{array}$

$\begin{array}{ll}\text { Interleukin-6 IL-6 } & \text { IL- }\end{array}$

Kappa (к)-opioid receptor $\quad$ KOR

$\begin{array}{ll}\text { Microtubule associated 1A/1B-light chain } 3 & \text { LC3 }\end{array}$

Mu ( $\mu$ )-opioid receptor $\quad$ MOR

Magneto-electric nanoparticle MENP

Mammalian target of rapamycin mTOR

Monocyte chemoattractant protein-1 MCP-1/CCL2

N-methyl-D-aspartate receptor $\quad$ NMDAR

Nuclear factor kappa-light-chain-enhancer of activated B cells NF-kB 
$\begin{array}{ll}\text { Parkinson's disease } & \text { PD }\end{array}$

$\begin{array}{ll}\text { Reactive nitrogen species } & \text { RNS }\end{array}$

$\begin{array}{ll}\text { Reactive oxygen species } & \text { ROS }\end{array}$

Regulated upon activation normal T-cell expressed and secreted RANTES

Sequestosome 1

SQSTM1/p62

Trans-activator of transcription

Tat

Tumor necrosis factor-alpha

TNF- $\alpha$ 


\section{CHAPTER 1: AN INTRODUCTION TO HIV-ASSOCIATED NEUROLOGICAL DISORDERS (HAND) AND AUTOPHAGY IN THE CONTEXT OF OPIATE ABUSE}

\subsection{Introduction}

Human immunodeficiency virus (HIV) is a lentivirus member of the Retroviridae family which causes systemic infection and can ultimately lead to acquired immunodeficiency syndrome (AIDS) [1]. Currently, over 36 million people worldwide are living with HIV/AIDS with approximately 1 million people dying of AIDS-related illnesses [2]. Characterized as an immune system disorder, the virus targets primarily $\mathrm{CD} 4^{+} \mathrm{T}$ cells as well as antigenpresenting cells such as macrophages and dendritic cells [3]. During infection, the HIV envelope glycoprotein gp120 interacts with the host CD4 receptor which induces a conformational change in the envelope protein allowing the gp41 protein to also bind host co-receptors C-C motif chemokine receptor 5 (CCR5) or C-X-C motif chemokine receptor 4 (CXCR4) found on the surfaces of antigen presenting cells and memory $\mathrm{CD} 4^{+} \mathrm{T}$ cells [4]. The viral selectivity or preference for one co-receptor over the other is termed as tropism, whereby R5-tropic viruses prefer the CCR5 co-receptor and X4-tropic prefer the CXCR4 co-receptor. Once infection is established, the virus is able to efficiently evade antiviral defense mechanisms while depleting $\mathrm{CD} 4^{+} \mathrm{T}$ cells and other immune cells, creating viral reservoirs, enhancing its own replication, and inducing the release of inflammatory cytokines in the process [5]. Despite all of this, it is not the systemic viremia that is responsible for the mortality due to HIV infection but rather the widespread exhaustion of the immune system leading to the increased susceptibility to opportunistic pathogens [6]. In addition, viral proteins which are produced and/or secreted by infected cells may trigger toxic effects to neighboring cells. These prospective apoptotic viral proteins including the envelope protein gp120, viral protein $R(V p r)$, viral infectivity protein 
(Vif), negative factor (Nef) and the transactivator of transcription (Tat), are important in mediating the infectivity and replication of the virus [7]. It was only with the advent and evolution of antiretroviral therapy that viral titers have become manageable leading to a drastic decrease in the mortality of infected persons.

Though HIV primarily affects the immune system, it can cross the blood brain barrier (BBB) during early infection and affect the nervous system [8,9]. The most well characterized mechanism is termed the "Trojan horse" theory where infected monocytes $\left(C D 14^{+} C D 16^{+}\right)$ cross the BBB due to increased response to chemokines such as CCL2 (also known as MCP-1) which are induced by viral presence in the CNS [10,11]. Within 4 to 8 days of peripheral infection, the virus enters the CNS and is spread to various other cell types including other monocytes/macrophages, microglia, endothelial cells, and to a lesser extent, astrocytes [12]. The productive and latent infection within these replicationcompetent cells, despite long term antiretroviral treatment, establishes and maintains the CNS as a viral reservoir capable of releasing neurotoxic viral proteins.

Despite the advances in combined antiretroviral therapy (cART), HIV infection in the brain remains persistent while causing a myriad of neurological problems including cognitive dysfunction, behavioral changes, and motor impairment. These neurological side effects of HIV infection in the nervous system are collectively known as HIV-Associated Neurological Disorders (HAND) and are seen in a percentage of the HIV infected population [13, 14]. In addition, certain trends in co-morbidities have emerged within subsets of HIV infected patients such as within the opiate using community. With approximately $30 \%$ of HIV infected persons being injection drug users (IDU), research has shown that the neural dysfunction and progression of HAND is accelerated in opiate drug 
abusers [15]. It is important to note that synthetic opioids are also used for analgesic treatment of neuropathic pain. Numerous mechanisms have been proposed to explain opiate exacerbation of HIV pathogenesis, most of which are based upon host pathways utilized by the virus. One such target of increasing interest is the autophagy pathway which is utilized by HIV to enhance its own viral biogenesis [16].

The term "autophagy" was created by Christian de Duve in 1963, winner of the 1974 Nobel Prize in Physiology for the discovery of lysosomes and peroxisomes [17]. Derived from the Greek auto- meaning "self" and phagein meaning "to eat", he described the observed cellular process wherein cytosolic organelles are delivered to the lysosome within doublemembraned vesicles as seen through electron microscope. Initially described as "focal cytoplasmic degradation," studies documented the multistage process in which sequestered components of cytoplasm were brought to lysosomes for the reutilization of cellular materials [18]. It was with de Duve's study of glucagon-induced cell degradation in the liver that lysosomes were recognized as the site of intracellular autophagy [19, 20].

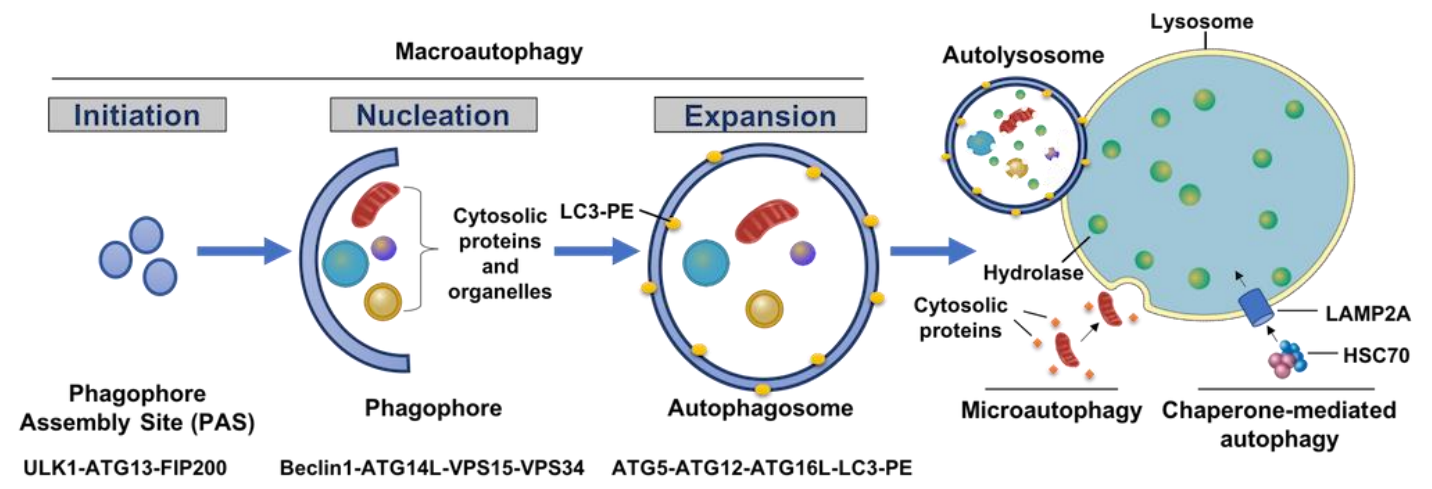

Figure 1.1 Summary of mammalian autophagy pathways.

Autophagy is classified as one of three types: Macroautophagy, microautophagy, and chaperonemediated autophagy. Macroautophagy (herein termed autophagy), can be broken down into 3 stages: initiation, nucleation, and expansion representing the activation of the pathway, early formation of the phagophore membrane, and elongation of the membrane to form the completed autophagosome. Recreated from [21]. 
The autophagy pathway can be characterized as one of three types (Figure 1.1; adapted from [21]), macroautophagy, microautophagy, or chaperone-mediated autophagy, each mediated by their respective autophagy-related genes, all of which terminate at the lysosome [22]. These three pathways predominantly differ in the locations where engulfment occurs, and the proteins involved in the process. The most studied of these is macroautophagy and will henceforth be referred to as autophagy. From its discovery in 1963 to today, the attention to autophagy in research has shifted from understanding of the mechanism to determining its roles in physiology and the pathogenesis of disease.

Numerous connections between autophagy and the pathogenesis of HIV are reported. The role of autophagy as an immune regulator provides a link between the pathway and the HIV immune response. As such, exploring that link between autophagy and HIV pathogenesis is of increasing interest. During HIV infection, autophagy was reported to be downregulated in both macrophages and dendritic cells, with macrophages and CD4 ${ }^{+} \mathrm{T}$ cells shown to accumulate autophagic vacuoles [16, 23]. The effects of HIV on autophagy in immune cells may provide the ideal state for viral persistence, immune evasion, and cell death in the CNS and periphery. Based on this research, autophagy is of particular interest for study because of its contributions to HIV neuropathology.

\subsection{Brief overview of the HIV genome}

As previously mentioned, HIV is a lentivirus belonging to the Retroviridae family and is transmitted as a single stranded, positive-sense, enveloped RNA virus [1]. Measuring about $100 \mathrm{~nm}$ in diameter, each virus contains a lipid bilayer viral envelope that encompasses the capsid, or protein shell, and the matrix which in turn hold the genetic information [24]. Within the capsid are two single strands of RNA which contains the virus's 
nine genes encoding 15 viral proteins, and the viral enzymes reverse transcriptase (RT) and integrase (IN) that are responsible for conversion of RNA to DNA and integration of viral DNA into the host genome respectively (Figure 1.2) [25-27]. The viral genome contains various structural and functional proteins and enzymes allowing for the replication and production of new viruses, but these have other roles in HIV persistence and pathogenesis.

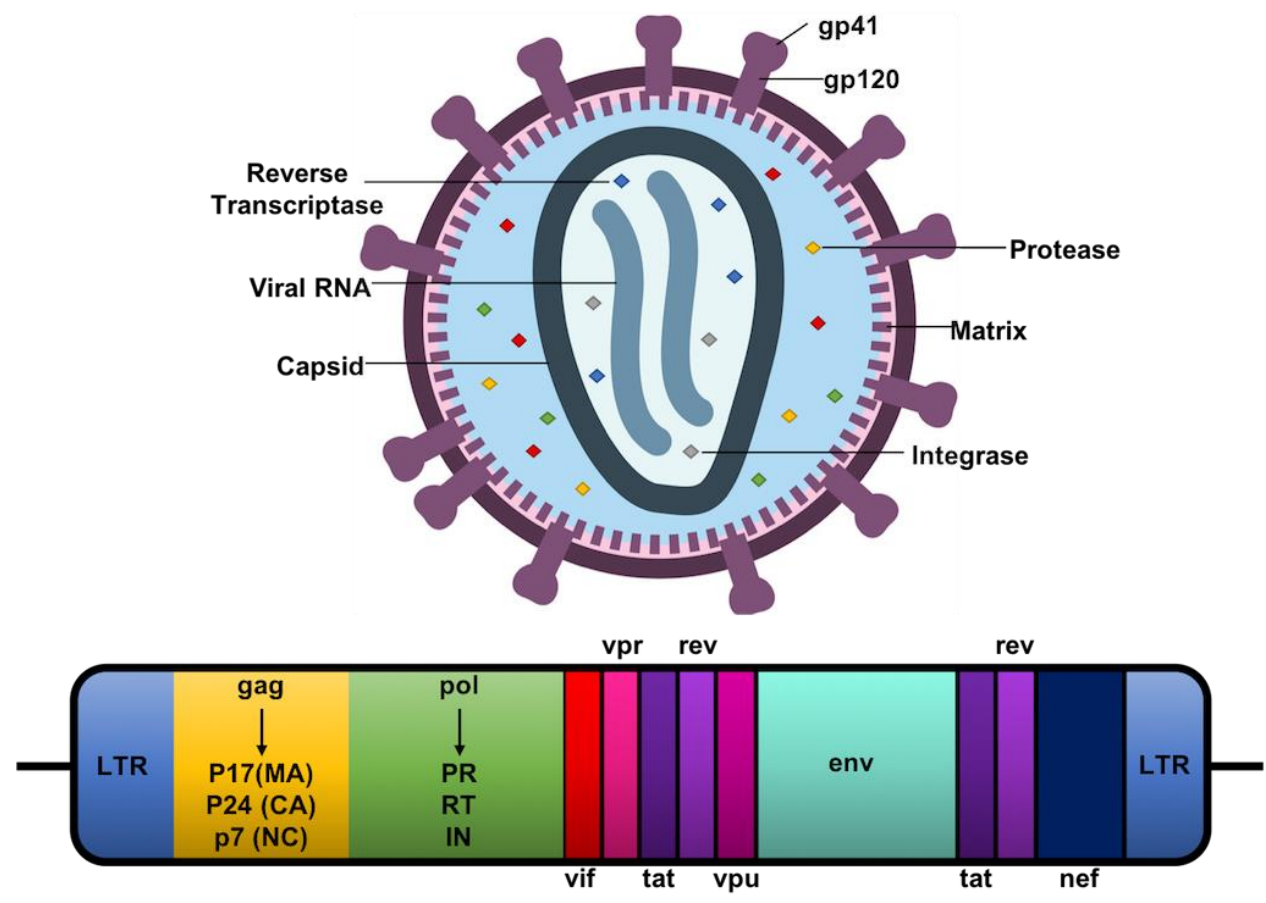

Figure 1.2 Diagram depicting components of HIV virion and the viral genome The HIV genome consists of 9 genes encoding 15 proteins utilized for viral replication and infection

The genes encoding the structural proteins include gag, pol, and env. Gag, or groupspecific antigen, codes for a precursor to the p55 gag polyprotein which is processed by the viral enzyme protease into the numerous structural peptides such as p17/MA, p24/CA, and $\mathrm{p} 7 / \mathrm{NC}$ which make up the matrix, capsid, and nucleocapsid proteins [26, 27]. The pol gene codes for numerous viral enzymes which are essential to viral infection, integration, 
and replication. These include the reverse transcriptase (RT), integrase (IN), RNase $H$, and protease (PR) enzymes, the last of which is used to process the primary protein products such as the gag proteins $[27,28]$. The env protein might be considered one of the most important structural proteins in terms of the study of HIV in the sense that it encodes the gp160 envelope protein which is the main determinant of HIV tropism and infectious capability. Cleaved by a host cell protease, gp160 is processed in the endoplasmic reticulum into two proteins, glycoprotein gp120 and transmembrane protein gp41, whose respective functions are for CD4 receptor binding and target cell attachment/fusion in HIV entry [27, 29]. The remaining genes encode both essential and accessory regulatory proteins which function to enhance viral reproduction (Table 1.1). These include the essential proteins, Tat and regulator of RNA splicing and transport (Rev), and the accessory proteins, Nef, Vpr, Vif, and virus protein $U$ (Vpu) [27]. Of these, it is the viral protein Tat which, due to its significant role in HIV replication and widespread effects in various tissues, will be the focus of the work presented.

Table 1.1 HIV gene products and functions

\begin{tabular}{|c|c|c|c|}
\hline & Gene name & Protein product(s) & Function \\
\hline \multirow{3}{*}{$\begin{array}{l}\text { Structural } \\
\text { proteins }\end{array}$} & gag & p55 gag polyprotein & Generates building blocks for the viral core structure \\
\hline & pol & $\begin{array}{l}\text { Reverse Transcriptase, } \\
\text { protease, integrase }\end{array}$ & $\begin{array}{l}\text { Conversion of viral RNA into DNA; processing of viral } \\
\text { proteins; integrates viral DNA copy into host DNA }\end{array}$ \\
\hline & env & $\begin{array}{l}\text { Envelope protein gp160 } \\
\quad(\mathrm{gp} 120 \text { \& gp41) }\end{array}$ & Viral attachment and embedding into host cells \\
\hline \multirow{2}{*}{$\begin{array}{c}\text { Essential } \\
\text { regulatory } \\
\text { proteins }\end{array}$} & tat & Tat & Regulates rate reverse transcription of viral genome RNA \\
\hline & rev & Rev & Regulates export of HIV mRNAs out of the nucleus \\
\hline \multirow{4}{*}{$\begin{array}{c}\text { Accessory } \\
\text { regulatory } \\
\text { proteins }\end{array}$} & nef & Nef & Negative regulator of HIV replication \\
\hline & $v p r$ & Vpr & Facilitates nuclear import of HIV pre-integration complex \\
\hline & vif & Vif & $\begin{array}{l}\begin{array}{l}\text { Enhances infectivity and synthesis of HIV particles in host } \\
\text { cells }\end{array}\end{array}$ \\
\hline & vpu & Vpu & $\begin{array}{l}\text { Aids in assembly and release of mature virions from } \\
\text { infected cells }\end{array}$ \\
\hline
\end{tabular}

Structurally, HIV Tat is a $14-15 \mathrm{kDa}$ protein which is generally expressed as either a $72-$ amino acid protein encoded by only one exon, or a 86-101 amino acid protein encoded by 
two exons; these differences are dependent on viral strain and play significant roles in HIV infectivity (Figure 1.3) [30]. In the early stages of infection, very low levels of RNA transcripts are made, allowing for small production of Tat protein. Despite the limited amount of Tat, it facilitates the rapid enhancement of viral replication. As such, it is one of the first proteins to be expressed after initial infection [31]. The approximately 10 amino acid basic domain of Tat, consisting of mostly arginine and lysine residues, binds to an RNA stem-loop structure near the 5' long terminal repeat (LTR) region which is known as the trans-activation response element or TAR region $[32,33]$. Binding to TAR activates the recruitment and interaction with a host protein complex named positive transcription elongation factor b (P-TEFb) which consists of cyclin-dependent kinase (CDK9) and cyclin T1 (CycT1). Tat also associates with RNA polymerase II complexes through CDK9 and it is through both of these interactions that allow Tat to increase the rate of whole-genome transcription and the production of full-length viral RNA [32, 34, 35]. Tat is often considered one of the most interesting viral proteins to study due to its multifaceted characteristics and functions. The basic domain of Tat is largely used for binding not only to TAR, but also various cell surface receptors which confer its pathogenicity $[31,36]$. The RGD domain found in exon 2 has also been shown to bind integrin receptors to facilitate cellular entry [37]. Additionally, Tat contains a protein transduction domain which allows for its passage across plasma membranes into cells, as well as a nuclear localization signal which facilitates further translocation into the nucleus [31, 38]. Given that Tat is actively secreted by HIV infected cells [39, 40], these characteristics make the protein a menace not only to infected cells, but also to bystander cells within the environment. 


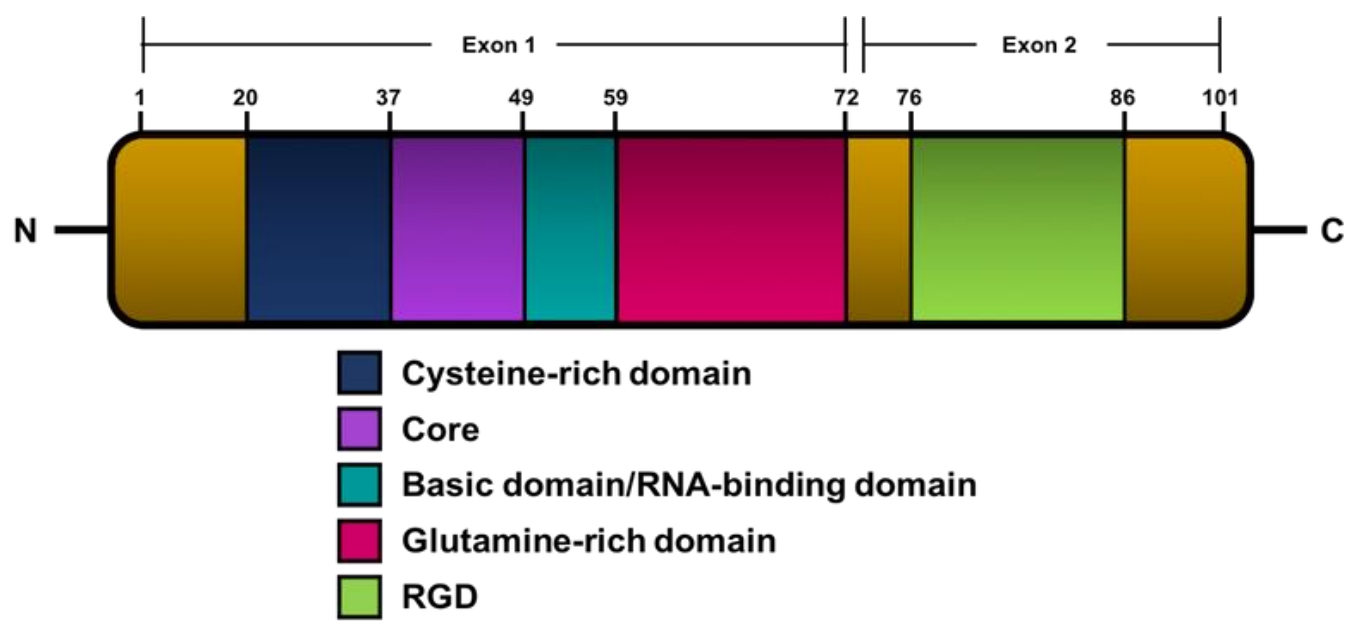

Figure 1.3 Functional domains of HIV Tat

HIV Tat protein comprised of an N-terminal proline-rich domain, a cysteine-rich domain, a core, a basic domain (basic), and a glutamine-rich domain within the 1-72 amino acid exon 1. Full length HIV Tat includes a second exon with an RGD (Arg-Gly-Asp) domain and contains 101 amino acids.

\subsection{HIV-Associated Neurological Disorders (HAND)}

The term "AIDS dementia complex" (ADC) was coined in 1987 by Navia and Price to describe complications affecting central and peripheral nervous systems in HIV-infected patients [41, 42]. Progressive encephalopathy from HIV infection in the brains of patients was detected and later called sub-acute encephalitis or HIV-encephalitis (HIVE), which is the main pathology recognized [43]. Following autopsy of HIV-infected patients' brain tissue, inspection revealed perivascular macrophage and lymphocyte infiltration, multinucleated giant cells, myelin loss, and white matter astrogliosis [44-47]. As imaging techniques improve, earlier diagnostic techniques for brain abnormalities through noninvasive imaging methods such as functional magnetic resonance imaging (fMRI), positron emission tomography (PET), and magnetic resonance spectroscopy (MRS) are gradually coming to the forefront [48]. AIDS dementia was associated with motor deficits and neurological impairments; however, the symptoms observed in patients were broad with varying levels of severity. As such, the term HIV-associated neurological disorders (HAND) was implemented to encompass the range of neurological deficits attributed to 
HIV infection in the CNS. HAND is thought to be a consequence of HIVE with its characteristic inflammation leading to cognitive, motor, and behavioral impairments. Clinically, HAND is differentiated into three categories based upon severity: asymptomatic neurological impairment (ANI), mild neurological disorder (MND), and HIV-associated dementia (HAD) being the most severe [49]. With the advent of combined anti-retroviral therapy (cART), the prevalence of HAD has drastically decreased; however, occurrence of ANI and MND have remained steady [50]. The risk of HAND increases with age, as do other risk factors such as cardiovascular disease, co-infection with hepatitis $\mathrm{C}$, and substance abuse [51]. There are varying effects of HIV infection in the CNS, depending on the cell type affected, which produce the characteristics of HAND (Figure 1.4).

\subsubsection{Microglial activation contributions to HAND}

Perivascular macrophages and microglia are the predominant actively infected cell types within the CNS and aid in viral persistence as a cellular reservoir for HIV [52-54]. As such, glial reactions to infection and uninfected bystanders putative contributors to the neurotoxicity and neuroinflammation in HAND [47]. Functionally, microglia survey the CNS environment and aid in the clearance of debris, accumulated proteins, or invading pathogens and are the primary source of inflammatory cytokines in the brain [55]. However, when infected by HIV, microglia facilitate the biogenesis, replication, and release of new virions while still producing proinflammatory cytokines such as IL-1 $1 \beta$, IL-6, TNF- $\alpha$, MCP-1 and IL-8 [56]. In addition to the production of virus, infected microglia secrete the viral protein Tat which is neurotoxic [57]. Tat mRNA, protein, and antibodies to Tat are detectable in the CNS of HIV-infected patients with higher levels observed in HAND [58-60]. Secretion of Tat into the cellular environment can propagate microglial activation and enhanced secretion of neurotoxic factors which may add to neuronal injury. 


\subsubsection{Astrocytic dysfunction in HAND}

Astrocytes are the most abundant cell type in the brain and serve to maintain an optimal microenvironment for neurons. The functions of astrocytes are varied including uptake of neurotransmitters, BBB maintenance, nutrient metabolism, growth factor secretion, and modulating synaptic transmission $[61,62]$. The extent of astrocyte infection is controversial because the level of virion production is markedly less than microglia [63]. Post-mortem studies looking at astrocyte activation following HIV infection have detected the presence of early HIV proteins such as Nef, Gag, and Rev which support the concept of astrocytic infection [64]. Regardless, the effects of HIV on astrocytes include impairing BBB function, HIV Tat inducing the production of cytokines, chemokines, and activation of nitric oxide synthase [65-68]. TNF- $\alpha$ released by activated microglia and astrocytes inhibits glutamate uptake in astrocytes through excitatory amino acid receptors. The buildup of extracellular glutamate can subsequently lead to neuronal excitotoxicity [69, 70].

\subsubsection{Cumulative effect of HIV infection on neurons}

In general, it is not believed that neurons can support HIV infection or sustain replication despite the expression of cell-surface receptors such as CCR5 and CXCR4 [71]. However, it has been well documented that the presence of these receptors still render neurons susceptible to injury by viral proteins such as Tat and gp120 and by glial-derived inflammatory cytokines, which may explain neuronal damage observed in HAND [72, 73]. In addition, neurons are subject to the fluctuations of the brain environment due to HIVinfection in other brain cells. As discussed above, microglia and astrocytes which are normally charged with the maintenance of homeostasis, can be dysfunctional upon HIV infection leading to the influx of inflammatory cytokines (IL-1 $\beta, I L-6)$ and accumulation of 
normally non-toxic substances (nitric oxide, glutamate) leading to excitotoxicity and neuronal death. For example, it has been shown that Tat and gp120 are able to interact with $\mathrm{N}$-methyl-D-aspartate receptors (NMDARs) which propagate excitatory signal transmission from glutamate binding through regulation of ion $\left(\mathrm{Ca}^{2+}, \mathrm{Na}^{+}, \mathrm{K}^{+}\right)$flux. The direct binding of Tat or gp120 to NMDARs on human neurons may cause lethal influx of calcium ions $[74,75]$. These viral proteins may have direct toxicity on neurons through

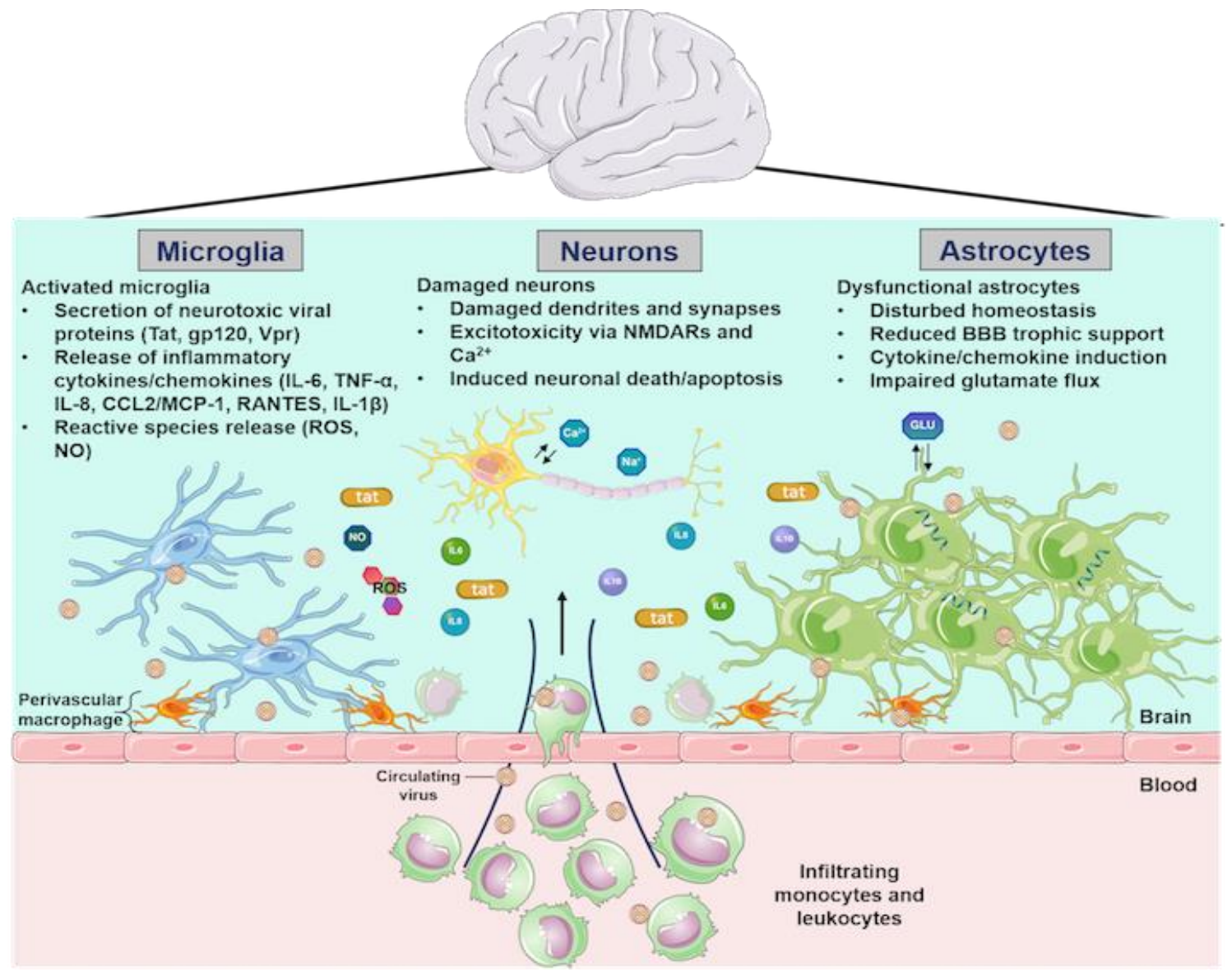

Figure 1.4 Overview of the effects of HIV on the CNS

HIV-infected monocytes and circulating virus cross the blood brain barrier to spread infection in the CNS. Infected macrophages and microglial cells release neurotoxic viral proteins that trigger astrocyte activation, which results in increased glutamate release and reduced glutamate uptake. Elevated extracellular glutamate levels cause neuronal ion imbalances that lead to aberrant synaptodendritic pruning and neuronal injury. Infection and viral protein presence lead to microglial activation and increased production of chemokines and cytokines and reactive oxygen species that contribute to neuronal injury. 
pathways such as mitogen-activated protein kinase (MAPK) p38 signaling cascades leading to caspase activation and apoptosis [76, 77].

\subsection{Interlinked epidemics between HIV and opiate drug abuse}

The use of injection drugs is a significant risk factor to the acquisition and spread of HIV due to the sharing of needles [78]. Those HIV infected individuals who use opiates for the treatment of neuropathic pain are also at risk for the exacerbation ofsevere HAND. Drugs of abuse (morphine, cocaine, and methamphetamine) have been reported to compromise the immune system which when in combination with HIV, leads to an exacerbated immune depletion. Studies have reported that drug users tend to have increased risk for HIV infection, poor adherence to and efficacy of cART, and increase viral replication [79]. With respect to HAND, drug abusers show a dramatic increase in neuroinflammation, oxidative stress, alteration in BBB integrity, and frequency of HIVE $[80,81]$. In addition, chronic exposure to opioids has been shown to worsen brain pathology, even in patients adhering to cART treatment, with symptoms of heightened CNS inflammation and increased cognitive deficits [82-84]. Clinically, patients chronically using opiates are found to have higher levels of microglial activation driving immune cell recruitment and neuroinflammation [85]. As such, the clinical characteristics of HIV infection in the brain can be accelerated and exacerbated by drug abuse [86].

Morphine is an opiate derived from the Papaver somniferum or opium poppy with analgesic characteristics that act directly on the CNS [87]. Within the brain, morphine is glucuronidated through the phase II metabolic actions of UDP-glucuronosyl transferase- 


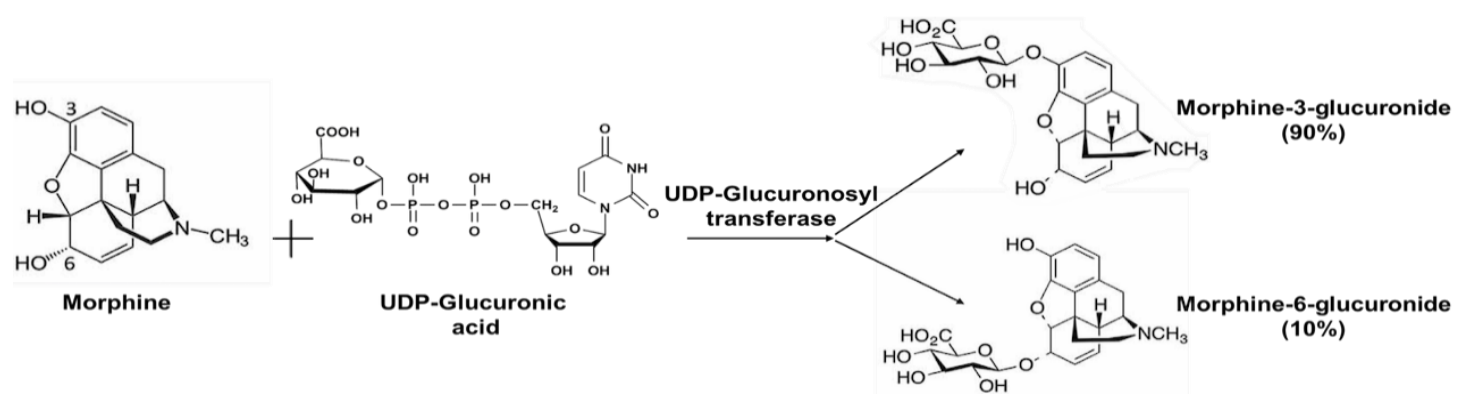

Figure 1.5 Morphine metabolism

Morphine is primarily metabolized in the liver by uridine-5'-diphosphate (UDP) glucuronosyltransferase and at nanomolar concentrations in the brain to morphine-3-glucuronide (M3G) and morphine-6-glucuronide (M6G).

2B7. Of the metabolites, morphine-3-glucuronide (M3G) and morphine-6-glucuronide (M6G) (Figure 1.5; adapted from [88]), only M6G is able to bind to $\mu$-opioid receptors (MORs) and have analgesic effects [89]. Structurally, morphine mimics endogenous opioids such as endorphins and binds as an agonist primarily to MORs located throughout the brain (with high densities in the caudate nucleus, putamen, hypothalamus and amygdala) and elsewhere [90, 91]. The three most well-known classifications of opioid receptor are the $\mu$ (mu), $\kappa$ (kappa), and $\delta$ (delta) receptors, with $\mu$-opioid receptor (MOR) having the strongest affinity for morphine, the $\delta$-opioid receptor (DOR) having a mild affinity, and the $\kappa$-opioid receptor (KOR) having little to no affinity [92, 93]. These opioid receptors are inhibitory G-coupled protein receptors (GPCRs) which respond to ligand binding by activating inhibitory $\left(\mathrm{G}_{\mathrm{i}}\right)$ protein second messengers which inhibit the activity of adenylate cyclase and prevent cyclic AMP (cAMP) production. Reduced cAMP leads to opening of $\mathrm{K}^{+}$channels while additional $\mathrm{G}$ proteins block influx of $\mathrm{Ca}^{2+}$ leading to hyperpolarization and reduced likelihood of an action potential. This quieting along pain sensory pathways ultimately leads to the sensation of pain relief or nociception depending 


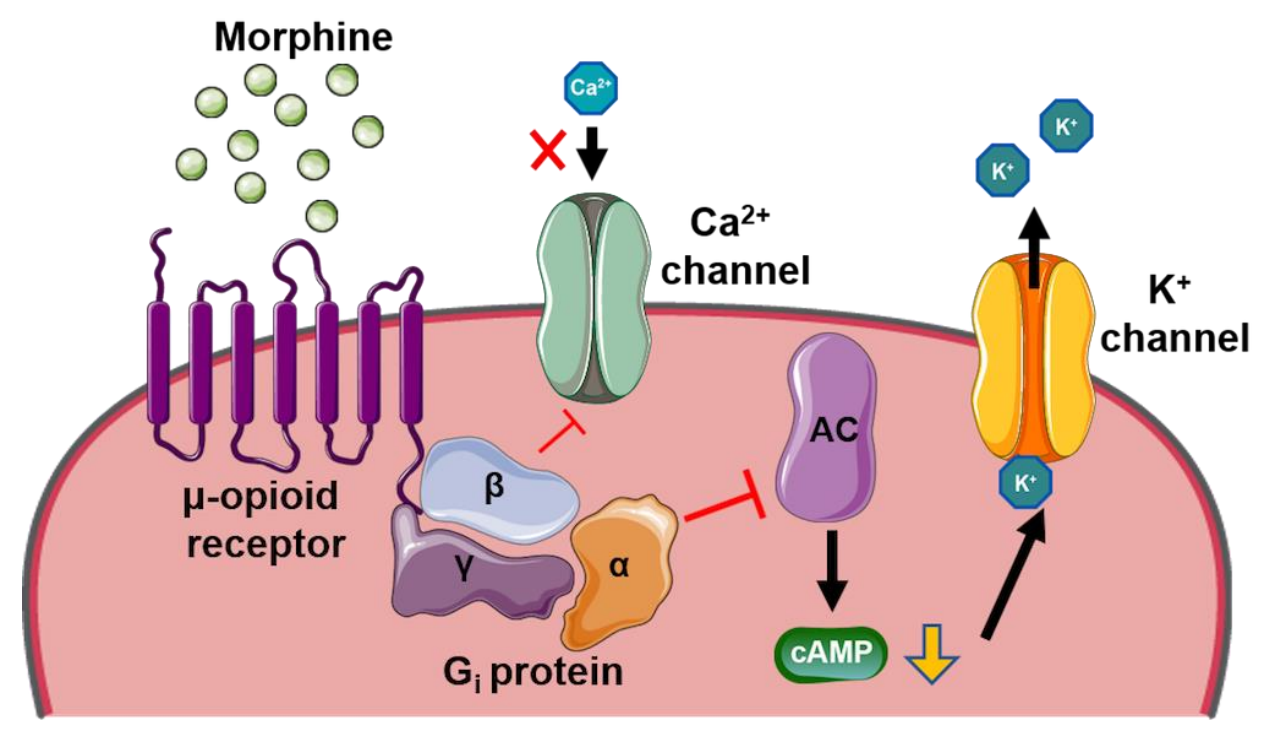

Figure 1.6 Mechanisms of opioid receptor signaling

Morphine binding to the opioid receptor causes dissociation of inhibitory G-proteins $G \alpha$ and $G \beta y$ subunits which subsequently act on effector proteins and channels. Agonist stimulation leads to Ga-mediated inhibition of cyclic adenosine monophosphate (cAMP) production and $\mathrm{K}^{+}$channel opening. $\mathrm{G} \beta Y$ subunit binding to $\mathrm{Ca}^{2+}$ channels cause channel closing leading to hyperpolarization of the cell. $A C=$ adenylate cyclase.

on the receptor activated (Figure 1.6) [94]. MOR activation is also associated with reward pathways of the dopaminergic system which constitute the risk of opiate abuse [95]. The DOR may supplement MOR activity given that it can also affect reward pathways; however, it can only modulate existing MOR activity and as such, has less risk for addiction. The KOR counteracts MOR/KOR activity and has an anti-reward effect [96, 97]. It is important to note that studies have found activation of opioid receptors in immune cells to modulate inflammatory processes which likely contributes to neuroinflammation [98]. Though a "typical" opiate abuser would be considered as an individual who abuses heroin, an alternatively derived form of morphine, there is rising abuse of prescription opiates [62]. Chronic opiate abuse was reported to advance neurodegenerative changes in the CNS similar to Alzheimer's disease (AD) from a clinical study in Edinburgh, UK [99]. As such, there is compelling evidence to investigate the interactions of HIV and opiate abuse in the context of HAND. 
In vitro studies with HIV/Tat and morphine have demonstrated how opioid exposure can play a key role in the enhanced pathogenesis of the virus within the CNS. As described earlier, HIV can show differing effects on the CNS depending on the cell type being targeted. Tight junction proteins such as ZO-1 and occludin are dysregulated when exposed to morphine alone or in combination with Tat indicative of BBB integrity compromise. This dysfunction was also associated with increases in intracellular $\mathrm{Ca}^{2+}$ within brain microvascular endothelial cells and increased transmigration across the BBB [81]. In striatal neurons, morphine has been presented to enhance HIV/Tat toxicity through caspase cascade mechanisms mediated specifically through opioid receptors $[100,101]$. Later studies showed that while HIV/Tat exposure in combination with morphine may have inherent toxicity to neurons, the most harmful of neurotoxic effects of are mediated through the activation of MORs on glial cells [102]. It is suggested that MOR agonists are able to potentiate the expression of the HIV co-receptors CCR5 and CXCR4 on peripheral and brain cells which facilitates viral entry [103]. Within infected glial cells such as microglia and astrocytes, HIV/Tat release triggers cytokine release, subsequent neuroinflammation, destabilizes intracellular ion homeostasis, and increases extracellular glutamate, ATP levels, and ROS/RNS which have all been shown to be modulated by morphine co-exposure [104-107].This disruption in glial utility culminates in reactive microglia and astrocytes which ultimately causes neuronal injury [54]. It is therefore clear that presence of HIV in the CNS has detrimental effects to normal function on numerous levels. In addition, the abuse of opioids potentiates this damage and hastens the acceleration to neurological impairment. Despite this knowledge, an underlying mechanism mediating this exacerbation remains unclear. Herein we provide evidence 
supporting the autophagy pathway as a potentially significant facilitator of HIV and opioid induced neurodegeneration.

\subsection{Mechanisms of Autophagy}

The process of autophagy is an evolutionarily conserved, highly regulated pathway in which cytosolic material including superfluous and damaged organelles, cytosolic proteins, and invasive microbes are degraded and recycled for components. This pathway is present at basal levels within all cells and acts as a response to increases in cellular stress as a means of avoiding cell death and an alternative to the ubiquitin-proteasome system. The defining feature of autophagy is the formation of the double-membrane bound phagophore and autophagosome (Figure 2) which when visualized using electron microscopy, acts as the gold standard for verifying activity [108]. The actions of autophagy are dependent on the genes and their respective proteins which have been categorized from studies in Saccharomyces cerevisiae. The autophagy machinery (Table 1.2; adapted from [108]) consists of over 34 autophagy-related genes (ATG) which have been identified from genetic studies in yeast, most having eukaryotic homologs with high protein sequence identity $[109,110]$.

The autophagy pathway can be divided into three morphological stages (Figure 2): induction, elongation and closure, and maturation. Induction is the first step in the pathway and is a consequence of a variety of signaling pathways acting on one of two targets, mammalian target of rapamycin (mTOR) and adenosine monophosphate activated protein kinase (AMPK). These protein kinases act as sensors for the cellular environment with AMPK being activated upon starvation conditions (and mTOR being inhibited) to directly modulate the phosphorylation of the unc-51-like kinase (ULK1/2) complex [111, 112]. 
Table 1.2 Mammalian autophagic machinery involved in autophagosome formation

\begin{tabular}{|c|c|c|}
\hline & $\begin{array}{l}\text { Mammalian } \\
\text { Protein }\end{array}$ & Characteristics \& Function \\
\hline \multirow{3}{*}{ ULK Complex } & ULK $1 / 2$ & Ser/Thr kinase; phosphorylated by mTOR; recruits ATG proteins \\
\hline & ATG13 & $\begin{array}{l}\text { Regulatory subunit; hyperphosphorylated by mTOR and/or PKA; interacts } \\
\text { with ULK } 1 / 2\end{array}$ \\
\hline & RB1CC1/FIP200 & Scaffold protein for ULK1/2 and ATG13; phosphorylated by ULK1 \\
\hline \multirow{5}{*}{ PI3K Complex } & Beclin 1 & Component of PIK3 complex \\
\hline & PI3KC3/Vps34 & PI3-kinase; phosphorylates PI3P \\
\hline & PI3KR4/Vps15 & Ser/Thr kinase \\
\hline & AMBRA1 & Component of PI3K complex; binds Beclin 1 \\
\hline & ATG14/UVRAG & Component of PI3K complex; binds Beclin 1 \\
\hline \multirow{3}{*}{$\begin{array}{l}\text { ATG9 Cycling } \\
\text { System }\end{array}$} & ATG2 & Interacts with ATG9 and WIPI 1/2 \\
\hline & ATG9 & $\begin{array}{l}\text { Transmembrane protein; directs membrane to phagophore; regulated by } \\
\text { ULK } 1 / 2 \text { activity }\end{array}$ \\
\hline & WIPI $1 / 2$ & PI3P-binding protein \\
\hline \multirow{4}{*}{$\begin{array}{c}\text { LC3 (ATG8) } \\
\text { Conjugation } \\
\text { System }\end{array}$} & LC3/GABARAP & Ubiquitin-like protein; conjugated to PE; recruited by PI3P \\
\hline & ATG7 & E1-like enzyme; binds ubiquitin-like proteins; conjugates LC3 to PE \\
\hline & ATG3 & E2-like enzyme \\
\hline & ATG4 & Deconjugating enzyme; cysteine protease; cleaves pro-LC3 \\
\hline \multirow{5}{*}{$\begin{array}{c}\text { ATG } 12 \\
\text { Conjugation } \\
\text { System }\end{array}$} & ATG12 & Ubiquitin-like protein; recruited by PI3P \\
\hline & ATG7 & E1-like enzyme; binds ubiquitin-like protein ATG12; activates ATG10 \\
\hline & ATG10 & E2-like enzyme; conjugates ATG12 to ATG5 \\
\hline & ATG16L1 & Interacts with ATG5 and ATG12 \\
\hline & ATG5 & Conjugated to ATG12 \\
\hline
\end{tabular}

Under nutrient-rich conditions, mTOR phosphorylates thus inhibiting the ULK1/2 and ATG13 complex activity which in turn disrupts the interaction between ULK1/2 and AMPK $[113,114]$. Alternatively, when autophagy is induced under starvation, mTOR is freed from the complex, leading to the activation of ULK1/2 and phosphorylation of ATG 13 and RB1CC1 [115]. Once the ULK 1/2 complex has been activated and translocates to the site of autophagosome formation, vesicle nucleation occurs which is characterized by the recruitment of the class III phosphatidylinositol 3-kinase (PI3K) nucleation complex consisting of Beclin 1, catalytic subunit type 3 (PIK3C3) also called vacuolar protein sorting 34 (Vps34), PIK3 regulatory subunit 4 (PIK3R4) also called vacuolar protein sorting 15 (Vps15), activating molecule in BECN1 regulated autophagy protein 1 (AMBRA1), ATG14 or ultraviolet radiation associated (UVRAG), and Bax-interacting factor 1 (BIF1; also known as SH3GLB1) [116]. Beclin 1 interacts with and is negatively regulated by the anti- 
apoptotic proteins $B C L-2$ and $B C L-X_{L}$ at the endoplasmic reticulum [117]. The displacement of $\mathrm{BCL}-2$ by $\mathrm{BCL}-2$ homology $3(\mathrm{BH} 3)$-only proteins or phosphorylation by c-Jun N-terminal kinase (JNK) causes the PI3K nucleation complex to be recruited to the induction site at the endoplasmic reticulum where it is phosphorylated by ULK1 [118]. Formation of the PI3K complex activates the kinase functions of PIK3C3 to phosphorylate phosphatidylinositol yielding the signaling lipid phosphatidylinositol 3-phosphate (PI3P) which is required for the assembly of the phagophore membrane [119].

The next step in the autophagy process is membrane elongation which uses two ubiquitinlike conjugation systems, the ATG5-ATG12/ATG16 complex and the LC3 complex [120]. In complex with WIPI 1/2 are ATG2 and the transmembrane protein ATG9 which conducts a membrane cycling system between peripheral sites and the phagophore assembly site or pre-autophagosomal structure (PAS) [121]. Under nutrient rich conditions, ATG9A localizes to the trans-Golgi network as well as to late endosomes. ATG9-positive membranes are shuttled to the phagophore assembly site for growth of the autophagosome based upon the activity of ULK $1 / 2[122,123]$. During this process of phagophore formation, the PI3P molecule recruits ubiquitin-like conjugation systems ATG5-ATG12/ATG16 complex and the LC3 complex [120]. ATG5-ATG12/ATG16 complex assembly is initiated by ATG7 acting as an E1-like enzyme on ATG12 followed by ATG10, an E2-like enzyme, which conjugates ATG5 to ATG12. ATG16 is also recruited and the complex localizes to the autophagosome elongation site. The LC3 complex contains microtubule associated 1A/1B-light chain 3 (LC3) and GABA receptor-associated protein (GABARAP) in addition to ATG7, ATG3 (E2-like enzyme), and ATG4. ATG4 is a cysteine protease and upon activation, cleaves pro-LC3 at its C-terminal glycine [124126]. The membrane lipid phosphatidylethanolamine (PE) is then conjugated to the newly 
cleaved soluble LC3-I by ATG7 and ATG3. This conjugation product of LC3-PE, now known as LC3-II, is subsequently incorporated into the inner and outer membranes of the autophagosome while it reaches closure [127]. The ATG5-ATG12/ATG16 complex also plays a role in the lipidation process of LC3 by acting as an E3-like ligase to enable LC3PE conjugation. Isolation membrane bound LC3-II acts as both a scaffolding protein and an anchor for the binding of autophagy adaptors which mediate selectivity [128].

Before autophagic clearance, ubiquitin binding proteins such as the adaptor protein sequestosome 1 (p62/SQSTM1) are tethered to the ubiquitin-like proteins LC3 or GABARAP and regulate protein cargo degradation [128-130]. Structures which have been targeted for degradation through autophagy are often ubiquitinated and bind autophagy receptors containing ubiquitin-binding domains and LC3-interacting regions (LIR) to encourage the cargos engulfment into autophagosomes [130]. P62/SQSTM1 is a widely multifunctional adaptor protein which is involved with biological processes such as removal of toxic protein aggregates and is indispensable for basal autophagy [131, 132]. With the autophagosome now whole and carrying its cytosolic cargo, it undergoes maturation during which the induction and elongation complexes dissociate from the membrane and can be delivered via microtubules upon close proximity to the lysosome [133]. The fusion of the autophagosome with acidic endosomes and lysosomes to form autolysosomes is mediated through soluble NSF attachment protein receptors (SNAREs) [134]. Once fusion has occurred, captured cargo is degraded by acidic hydrolytic enzymes and proteases within the lysosome yielding macromolecule components which can be recycled for alternative use [108]. 


\subsection{Importance of Beclin 1 in autophagy}

Given that autophagy is a largely complex process with a number of contributing proteins, it is important to determine which proteins may play the most significant roles. In particular, the studies of this dissertation focus on the initiation protein Beclin 1. The mammalian Beclin 1 is encoded by the $B E C N 1$ gene with the yeast equivalent being autophagy-related gene 6 (Atg6) [135]; it plays a central role in autophagy and is also described as a haploinsufficient tumor suppressor $[136,137]$. Notably, Beclin 1 participates in various other signaling pathways such as endocytosis, phagocytosis, vacuolar protein sorting, and cytokinesis, independent of its role in autophagy [138]. As previously described, Beclin 1 acts as a subunit within the PI3K complex during autophagy initiation to mediate the formation of phosphatidylinositol 3-phosphate [129]. Much of its functional significance lies in its many interacting partners which are generally associated with formation of autophagosomes or with endocytosis. The mammalian protein consists of three domains: a central coiled-coil domain (CCD), an evolutionarily conserved domain (ECD) and a BH3only domain (Figure 1.7 adapted from [138]). The CCD domain of Beclin 1 interacts with the CCD of UVRAG, a positive regulator of autophagy. This interaction allows Beclin 1 to act as a type scaffold protein to allow UVRAG contact with PI3K and other proteins, promoting starvation-independent autophagy [139]. The ECD of Beclin 1 is essential for binding another autophagy protein Vps34 as well as its tumor suppressing functions [140]. The BH3-only domain ties Beclin 1 to the BCL-2 family of proteins which are pro-survival in function and sense cell damage or dysfunction. This domain interacts with the $\mathrm{BH} 3$ domains on BCL-2 and BCL-X $X_{L}$ and inhibit autophagy by blocking Beclin 1 interaction with PI3K; however, this interaction is largely diminished upon starvation [141]. Nutrient starvation, which induces autophagy, can stimulate the dissociation of Beclin 1 from its inhibitors, either by activating $\mathrm{BH} 3$-only proteins or by phosphorylation of BCL-2 that may 
reduce its affinity for Beclin 1 and $\mathrm{BH} 3$-only proteins. As such, anti-apoptotic BCL-2 family members and pro-apoptotic $\mathrm{BH} 3-$ only proteins may participate in the inhibition and induction of autophagy, respectively Despite being a $\mathrm{BH} 3-$ only protein which are proapoptotic, Beclin 1 is considered to be pro-survival [142]. Studies with Beclin 1 mutants have displayed the importance of the protein to numerous processes, particularly embryonic development, tumorigenesis, and protein aggregation diseases. Where homozygous beclin $1^{-/}$mice die early in embryogenesis, heterozygous beclin $1^{+/-}$mice have not only decreased autophagy, but also increased incidence of spontaneous tumors [136, 137].
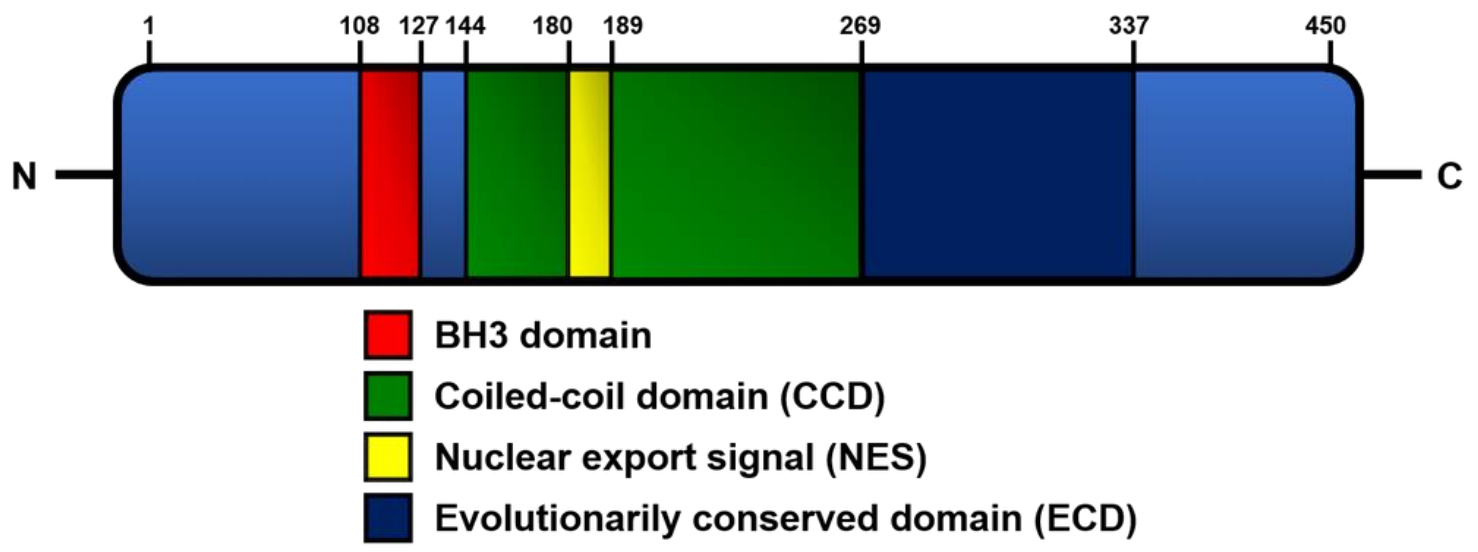

Figure 1.7 Functional domains of the autophagy protein Beclin 1

Beclin 1 contains distinct functional domains: $\mathrm{BH} 3$ interacting domain, coiled-coil domain, nuclear export signal, and evolutionarily conserved domain. Adapted from [138]

\section{$\underline{1.7 \text { Functions of Autophagy }}$}

\subsubsection{Autophagy and basal metabolism and homeostasis}

The autophagy process can be described as either non-selective or selective dependent on the cargo being taken up: non-selective being used for the turnover of bulk cytoplasm and selective autophagy targeting specific organelles such as mitochondria (mitophagy), peroxisomes (pexophagy), or clearance of microbes (xenophagy) [108]. The basal 
functions of autophagy serve to enhance cellular survival by adapting to fluctuations in nutrient conditions as well as to regulate cellular energy homeostasis. In the process, the pool of cellular precursors is replenished by degrading cytosolic proteins in response to starvation and nutrient depletion [143]. These catabolic products include amino acids, lipids, and sugars to be utilized for generation of new proteins, membranes, gluconeogenesis, ATP, nucleotide and carbohydrate synthesis, and glycolysis [144]. In the liver, amino acids generated through autophagy can be used for gluconeogenesis to maintain systemic glycemia under starvation conditions while autophagic degradation of liver lipid droplets also produced free fatty acids from triglycerides $[145,146]$. ATP can also be generated by way of newly produced lipids and amino acids entering the tricarboxylic acid cycle and oxidative phosphorylation or through sugars undergoing glycolysis which also maintains sugar levels [144]. However, these functions can be abused in disease conditions such as cancer. Due to their rapid replicative characteristics, tumor cells exhibit metabolic stress additionally caused by decreased nutrient availability and hypoxic conditions. To counteract these stressors, tumors and their surrounding fibroblasts couple and reprogram their metabolism to use autophagy as a protector by reducing oxidative stress and maintaining genomic stability [147]. Additionally, the breakdown of glutamine in cancer cells yields ammonia which has been shown to also stimulate autophagy in nearby cells [148]. Metabolic coupling has also been seen under normal conditions in the brain where astrocytes and neurons exchange metabolites to maintain homeostasis $[149,150]$. As research in autophagy has expanded, other functions have emerged beyond maintaining cellular homeostasis including the maintenance of mitochondria, cell differentiation and development, monitoring of unfolded proteins, protein secretion, and the trafficking of proteins to the plasma membrane [151-153]. 
Autophagy can be induced as a response to cellular stress from external stimuli such as oxidants, hypoxia, infectious agents, and stressors of the endoplasmic reticulum [154]. The degradative capabilities of autophagy are particularly important for the clearance of damaged and/or aggregated proteins and organelles, especially in situations where the proteasome clearance pathway has been impaired or overwhelmed [155, 156]. Genetic studies with mice have shown the importance of autophagic intracellular quality control of proteins. Deletion of autophagy genes such as $\operatorname{Atg} 5$ or $\operatorname{Atg} 7$ in the mouse liver induced the cytoplasmic accumulation of abnormal ubiquitinated proteins and organelles [157, 158]. In addition, liver-specific Atg $7^{-1-}$ mice have been shown to develop hepatomegaly and hepatic failure illustrating the crucial role of autophagy in locations of high protein turnover such as the liver. Ubiquitin accumulation has also been reported in other tissues such as some endocrine glands, the heart, skeletal muscle, and kidneys; however, to a lesser degree $[158,159]$.

\subsubsection{Autophagy in apoptosis induction}

Outside of the pro-survival functions of autophagy, there are correlations to the induction of apoptosis which are not completely understood. Studies have observed an accumulation of autophagosomes preceding cell death which was originally termed autophagic cell death [160]. However, studies have been varied with autophagy being able to act as both a protagonist and antagonist of apoptosis, largely dependent on the cellular context and environment. Many links between apoptotic signaling and autophagy lie in the crosstalk between pro-apoptotic signals which can induce autophagy as well as the binding of Beclin 1 to pro-survival protein BCL-2 [141, 161]. Studies on mice lacking the $\mathrm{BH} 3$ proteins $\mathrm{BAK}$ and $\mathrm{BAX}$ which regulate the release of mitochondrial proteins during apoptosis demonstrated that upon exposure to DNA damaging stimuli, cell were still able 
to die through autophagic cell death mediated by ATG5 and Beclin 1 [162]. In fact, a number of the ATG genes which regulate autophagy also regulate apoptosis regulators such as p53 and the death inducing signaling complex (DISC) [163]. Under normal conditions, autophagy plays several maintenance roles in a variety of tissues; however, as research in the topic expands, its roles in disease states are gradually coming to light. The formation and accumulation of protein aggregates has been associated with several human diseases known as conformational diseases or proteopathies. In these diseases, a particular protein may be misfolded as a result of mutation, improper clearance through either the proteasome or autophagy pathways, or defective chaperones [155]. These misfolded proteins tend to form aggregates leading to cellular dysfunction in organs such as the lungs, liver and brain [156].

\subsubsection{Autophagy in infectious diseases}

The roles of autophagy in the adaptive and innate immune systems have been extensively studied over recent years with functions including the clearance of pathogens, facilitation of antigen presentation, and regulation of the inflammatory response $[164,165]$. In particular, the clearance of potentially threatening intracellular pathogens through autophagy called xenophagy, acts as an additional defense mechanism [166]. While pathogens such as bacteria Mycobacterium tuberculosis and Pseudomonas aeruginosa and viruses like herpes simplex virus type 1 (HSV-1) are identifiable as substrates for autophagic clearance, a number of pathogens have evolved mechanisms of evasion to avoid degradation or manipulation to support growth such as HIV and hepatitis B and C viruses [164]. In addition to direct action on invading pathogens, autophagy can also regulate inflammation through signaling cascades of the inflammasome within myeloid cells of the innate immune system. This can result in modulation of cytokines such as IL1 $\beta$, 
IL-18, and IFNy, activation of natural killer cells, and nuclear factor kappa-light-chainenhancer of activated B cells (NF-kB) signaling [167]. Though stimulation of autophagy may not be required for degradation, these cascades may be initiated by their targeting to the autophagosomes formed by basal autophagy. The contribution of autophagy to the functions of immune cells is widely dependent on cell type due to varying levels of basal autophagy; however, it is of particular importance to antigen-presenting cells which have the highest levels of basal autophagy [168]. Regardless, in most immune cells, autophagy aids in cellular survival by avoiding apoptotic or inflammasome activated pyroptotic mechanisms through the mounting of a strong autophagic response [169, 170].

Despite the role of autophagy in immunity and pathogen clearance, the pathway's interactions with HIV are complex due to the variety of cells affected which may or may not be infected. Dendritic cells, for example, have drastic downregulation of autophagy when exposed to viral envelope proteins by activating mTOR which results in protection of the virus from autophagy-mediated degradation and subsequent increase in $\mathrm{CD} 4^{+} \mathrm{T}$ cell infection [171]. This study also showed that inhibition of autophagy can impair toll-like receptor (TLR) mediated innate responses, affect antigen processing, as well as MHC class II antigen presentation to $\mathrm{CD} 4^{+} \mathrm{T}$ cells. Studies with macrophages infected with HIV show an increased level of autophagic vacuoles and lack the viral envelope proteinmediated manipulation of the autophagy pathway [23]. Further mechanistic studies showed that despite the preservation of the initial stages of autophagy in HIV-infected macrophages, the autophagosome maturation process is inhibited by the HIV viral protein Nef [16]. This protein which assists in viral replication has been shown to interact with Beclin 1 which inhibits the maturation stages of autophagy and prevents lysosomal degradation of HIV while promoting biogenesis thereby enhancing viral yields. Conversely, 
uninfected macrophages do not show envelope protein induction of autophagy but are instead affected by Tat to activate Src-AKT and STAT3 or suppress STAT1 (through IFNY inhibition) pathways to culminate in autophagy repression $[172,173]$. Since macrophages are generally not susceptible to HIV mediated cell death, autophagy can also play a role in the promotion of macrophage viral reservoirs [3].

$\mathrm{CD}^{+} \mathrm{T}$ cells can have slightly different responses to HIV infection and thus different effects on autophagy which makes many studies inconclusive. Many $\mathrm{CD} 4^{+} \mathrm{T}$ cells appear to remain uninfected yet still die through what is termed bystander T cell death which is thought to be mediated by the host's responses to viral infection leading to chronic inflammation [6,174]. When uninfected bystander T cells were co-cultured with cells stably expressing either R5- or X4-tropic envelope proteins, they tended to favor undergoing apoptosis and accumulation of autophagic vacuoles; however, when productively infected with R5- or X4-tropic HIV, these T cells had diminished accumulation of autophagic vacuoles $[23,175]$. It is necessary to note that the study does not distinguish the cause of autophagic vacuole accumulation between autophagy induction or defects in autophagic maturation, so the precise cause of apoptosis is yet to be determined. Nevertheless, this supports the idea that the virus has evolved mechanisms to evade the antiviral functions of autophagy [176]. For example, in infected T-cells the viral protein Vif is able to block envelope protein induced autophagy typically seen in bystander $\mathrm{T}$ cells to prevent the autophagic degradation of Tat and allow productive infection $[177,178]$. At the clinical level, in peripheral blood mononuclear cells (PBMCs) of HIV-infected persons termed HIV controllers i.e. those who remain asymptomatic for many years or have viremia below detection limits, have a significant increase in autophagic vacuoles with elevated mRNA levels of Beclin 1, as compared to normal progressors [179]. Though the interactions 
between HIV pathogenesis and the autophagy pathway within the periphery may be complex, it provides a foundation for the study of the role of autophagy in HIV infection within the brain and the pathogenesis of HAND.

\subsection{Autophagy and Neurodegeneration}

Several neurodegenerative diseases have been shown to have dysregulations in the autophagy pathway [180]. Although autophagy is not induced in the central nervous system under starvation conditions, it plays a significant role in protein quality control maintenance and cellular homeostasis [181]. In general, autophagic activity within the brain is low but the conservation of basal autophagy is particularly essential to the CNS in the case of neurons owing to the fact that they are post-mitotic and rely heavily on clearance mechanisms such as autophagy for cell survival [182]. The ability of neurons to survey cellular contents for altered proteins or damaged organelles and process their degradation is essential to the prevention of intracellular buildup and eventual neurotoxicity [183]. Neural specific $\operatorname{Atg} 5$ and $\operatorname{Atg} 7$ knockout mice show accumulation of abnormal ubiquitinated proteins within neurons along with neurodegeneration and progressive motor deficits [158, 184]. Development of molecular probes such as LC3tandem sensors, and tracking methods such as lysotracker in combination with pharmacological inhibitors have allowed for the identification and better understanding of the individual stages being affected in diverse neurodegenerative disease. These include defects in autophagy induction, enhancement of autophagy repression, alterations in cargo recognition, inefficient autophagosome/lysosome fusion, or faulty degradation of the autophagic content within the lysosomes [180]. Comprehending when and where the autophagic halt is occurring in these neurodegenerative diseases is essential for the targeting of the autophagy pathway in potential therapeutics. Presently, the best studied 
examples of proteopathies include two of the major neurodegenerative diseases: Alzheimer's (AD) and Parkinson's (PD) disease both of which have correlates to malfunctions in autophagy. Genetic studies using mice with defective autophagy proteins have shown age-dependent neurodegeneration coinciding with the accumulation of protein aggregates [184]. In addition, the use of pharmacological agents which induce autophagy have been shown to inhibit symptoms associated with neurodegeneration in mouse models of AD and PD [155]. Given that autophagy is found to be altered in a number of the 'classical' neurodegenerative diseases, it's roles in other neurological disorders is of interest for study.

\subsection{Autophagy in HAND}

In terms of HAND, dysregulation of autophagy has been shown in the CNS in a number of studies. When the post-mortem brains of patients showing HIVE were analyzed for fluctuations in autophagy proteins, ATG5, ATG7, Beclin 1, and LC3-II were found to be increased as compared to non-HIVE tissue samples which showed no change in autophagy level. The same study also showed an increased number of autophagosomes and lysosomes indicative of autophagic blockade prior to degradation [185]. Later studies suggest that these characteristics are age dependent with aged HIVE patients showing more extensive neurodegeneration and reduced autophagy as compared to younger HIVE patients which showed increased autophagy markers [186]. Dever et al also demonstrated the changes to ATG gene expression in post-mortem brain tissues of HIV infected patients presenting with or without neurocognitive impairment $(\mathrm{NCl})$ or encephalitis and suggested that these genes may be differentially regulated at the mRNA or protein level within the brain based on infection stage [187]. 
In neuronal cultures, exposure to HIV lead to increase of autophagosomes and suppression of autophagy [188]. Other studies also showed a decrease in neuronal autophagy when exposed to the supernatant of infected microglia indicating the indirect neurotoxicity neurons are subjected to $[187,189,190]$. When exposed to Tat, there was a dose dependent decrease in the autophagosome markers LC3-II and p62/SQSTM1 in neurons suggesting Tat increases autophagic degradation which when unchecked, can lead to autophagic cell death [191]. The same study saw in Tat transgenic mice, Tat expression caused an increase in autophagosome accumulation within neurons along with neurodegeneration which was largely associated with lysosomal-associated membrane protein 2A (LAMP2A).

The autophagy pathway has been shown to be induced in microglia when infected by HIV with increased conversion of LC3-1 to LC3-II and increased expression of Beclin 1 and ATG5. This was also associated with accumulation of autophagosomes and increases in cytokines and chemokines [192]. Induction of autophagy has also been shown upon Tat treatment in glial cells by increasing the anti-apoptotic/pro-autophagic factor BCL2associated athanogene 3 (BAG3) levels [193]. While autophagy induction might seem beneficial, HIV has evolved ways to block the maturation of the pathway to prevent lysosomal degradation. For example, the viral protein Nef was shown to mimic the fusion inhibitor bafilomycin A1 and prevent autophagic degradation which can ultimately lead to increased viral presence [194]. This induction and later blockade has also been shown in astrocytes [188]. Given these results, it can be said that the role of autophagy in HAND is a complicated one with effects being cell type dependent and specific to particular stages of the autophagy pathway. 


\subsection{Interplay between HAND, opiates, and autophagy}

While the characteristics of HAND and the opiate induced exacerbation of its neuropathology have been widely studied and reviewed, the underlying mechanisms which lead to these effects are still unclear. Based on the interactions between HIV and autophagy previously discussed, it is possible that the degradative pathway converges on the HIV and opiate interaction. Despite limited literature on the interactions of autophagy and morphine, studies have seen that chronic morphine or morphine addiction activates the autophagy pathway in rodent hippocampus $[195,196]$. In these studies, chronic morphine treatment was associated with cell death and decreased mitochondrial DNA copy number which were mediated by autophagy. A separate study showed that morphine was able to magnify LPS-induced initiation of autophagy; however, similarly to what is seen in HIV, the later stages of autophagosomal maturation are halted thus inhibiting pathogen clearance [197]. This was also shown in neurons which were exposed to the supernatant of HIV infected microglia treated with morphine resulting in a significant elevation of p62/SQSTM1 expression suggesting the inhibition of autophagic clearance [187]. Taken together, if the morphine inducing effects on autophagy parallel the induction of autophagy though HIV infection, this may explain the phenomenon of opiate and HIV interaction while providing the autophagy pathway as an underlying mechanism and potential target for intervention.

\subsection{Modeling HAND in mice}

In the efforts to further study the mechanisms and pathology of HAND, one of the most useful tools has been the use of animal models. Non-human primate models of HAND have been utilized to understand the neuropathogenesis of HAND; however, there are limits to gaining mechanistic insight into HAND as well as high cost for maintaining these 
animals. Alternatively, rodent models have been shown to be highly useful in the study of HAND for their low cost, ease of use, and capability for genetic manipulation in the understanding pathological mechanisms at work.

\subsubsection{Transgenic mouse models}

The generation of transgenic mouse models have provided a wealth of information on the roles of HIV genes and their corresponding gene product functions in mediating HAND pathogenesis. Often these mice possess either constitutive or inducible expression of HIV proteins allowing researchers to study the effects of continuous exposure to viral proteins. This can be seen as representative of patients under cART regimen which have systemic viral replication under control but still have the production of viral proteins from viral DNA integrated cells or viral reservoir locations such as the brain [198]. Most cART drugs target the protease or reverse transcriptase actions of HIV and do not prevent the early production of viral proteins such as Tat, Rev, and Nef [7].

\section{(a) Tat transgenic mouse model}

Tat transgenic mouse models were established by Jones et al shortly after the effects of intraventricular injection of human HIV Tat into mice brains led to inflammation, gliosis and apoptosis [199]. In human infection, Tat is the first viral protein to be secreted and is largely able to cross cell membranes with studies of its effects being tissue specific. In the early studies, transgenic mice with constitutive expression of Tat in most peripheral tissues showed elevated levels of Tat in serum; however, these studies were unable to detect Tat in the brain and lacked neurological impairment [200-203]. Subsequently, two brainspecific Tat transgenic mouse models were generated from independent labs which placed Tat expression under the control of a glial fibrillary acidic protein (GFAP) promoter 
which could be induced in astrocytes by doxycycline administration and showed neurological abnormalities [204, 205]. The GFAP-Tat transgenic mouse generated by Kim et al showed damage induced by Tat expression to be largely localized to the cerebellum and cortex [205]. The damage induced by Tat expression in the Fitting et al. study was more understated with observations of reduced neuronal spine density and dendritic pruning [204]. Later studies also showed deficits in learning and memory accompanied with synaptic dysfunction and suppression of long-term potentiation [206, 207]. In addition, Tat transgenic mice have been used to study blood brain barrier integrity and show barrier destabilization along with immune cell recruitment [208]. In the context of opioids, HIV Tat transgenic mice administered morphine showed not only an alteration in analgesic efficacy, but also increases in microglial and astroglial activation [100, 102, 209]. In addition, Tat transgenic mice treated with morphine revealed significant decreases in the density of dendritic spines within the striatum [204].

(b) Gp120 transgenic mouse model

The envelope protein gp120 is shed as a soluble protein in the brain by macrophages and astrocytes which can also interact with and cause damage to neurons. The desire to understand its role in mediating neurotoxicity led to the generation of two distinct transgenic mouse models which expressed the gp120 encoded section of the env gene under control of a promoter [210, 211]. The Berrada strain expressed viral gp160 under the promoter control of human neurofilament light chain $(\mathrm{NfL})$ in neurons within the brain stem and anterior horns of the spinal cords in addition to exhibiting dendritic swellings on motor neurons. However, this strain showed no expression to be detected within the cerebral cortex [211]. The Toggas strain has been well studied and expresses soluble gp120 in the brain under control of the astrocyte specific GFAP promoter. Gp120- 
transgenic mice illustrated the protein's neurotoxic effects with pathological damage to the neocortex and hippocampus which was strikingly similar to that seen in human AIDS brains [210]. These mice displayed decreased synaptic and dendritic density, neuronal loss, as well as microglial activation. Additional studies have shown that these mice have dysfunctional differentiation and proliferation of neural progenitor cells, abnormal shortand long-term potentiation, and deficits in spatial retention and swimming velocity [212215]. Neuronal injury in these models have been suggested to be mediated by the enhancement of glutamate signaling through NMDARs and excessive activation of $\mathrm{Ca}^{2+}$ signaling pathways [216, 217].

(c) Transgenic rat model

As an alternative and more comprehensive means of studying the effects of viral proteins on the brain, the HIV transgenic model was generated which incorporates the HIV genome directly into the rodent model. These rodents express all but 2 of the 9 HIV viral genes (gag and pol) which in turn render them non-infectious and useful for mechanistic studies of viral protein effects [218]. The non-infectious pro-virus pEVd1443 was first used to generate an HIV transgenic mouse; however, these mice had inconsistent distribution of the transgene, inefficient tat transactivation, and limited clinical manifestation typical of HIV exposure $[35,219,220]$. Later, the pro-virus was introduced into the fertilized egg of F344 and Sprague Dawley bred rats with the transgene being incorporated hemizygously $[35,220]$. Despite no viral replication within these rats, the HIV viral proteins are continuously expressed throughout the life of the animals within a number of tissues including the lymph nodes, spleen, and brain [221]. Studies utilizing this model have reported alterations in sensorimotor gating, cognition, dopamine function, and neuroinflammation with marked decrease in specific brain areas such as the subcortex 
[221-223]. In terms of substance abuse, studies with morphine have suggested that HIV transgenic rats exhibit higher anti-nociceptive effects than normal controls in conjunction with elevated expression of mu-opioid and chemokine receptors which can in turn be modulated by cytokines $[224,225]$. Later studies also showed an elevation of MOR expression in HIV transgenic mice which was further elevated under LPS-induced immune challenge [226].

\subsubsection{Humanized mouse models}

Humanized mouse models for the study of HIV have been generated using immunodeficient mouse strains such as severe combined immunodeficiency (SCID) mice. These mice lack T and B lymphocytes and are incapable of mounting regular adaptive immune responses. As such, it is possible to directly inject HIV-infected human monocytes into the CNS of these immunocompromised mice [227]. Following stereotaxic injection into the basal ganglia [228], putamen or cortex [229], infected macrophages are able to express HIV p24 antigen as well as present the histopathological features of HIVE [229]. This includes infection of perivascular macrophages, astrocytosis, microglial activation, neuronal apoptosis, and dendritic damage [230]. These mice have also been used to study potential therapeutics for treating HIV-associated neuroinflammation. Additional humanized mouse models have also been proposed for further study of the physiological effects of HIV. The humanized immune system or HIS model uses immunodeficient mouse strains such as the NOD/Shi-scid IL2ry ${ }^{-1-}$ (NOG), NOD/LtSz-scid IL2ry ${ }^{-1-}$ (NSG), or $\mathrm{BALB} / \mathrm{cRag} 2^{-1} \mathrm{Y}_{\mathrm{c}}{ }^{-1-}$ (BRG), all of which show defects in innate and adaptive immunity, and intravenously injecting CD34+ hematopoietic stem cells [231-233]. These cells mature into human immune cells which can later be infected with HIV and sustain infection; however, these models do not contain HIV-infected microglia or astrocytes. Jaeger and Nath 
proposed a HIS-CNS model which suggests transplantation of both the human immune system and HIV-permissive cells of the human brain [227]. This would allow for the study of concurrent peripheral and CNS HIV-1 infection as well as the neuropathological characteristics of HAND. Studies which injected CD34+ hematopoietic stem cells into the brain were able to observe marked neuroinflammation, HIV replication in human mononuclear phagocytes, as well as encephalitis. Separately, NSG mouse transplantation with fetal brain cell cultures showed inclusion of human microglia in NSG mice [234].

\subsubsection{Direct viral-injection models}

(a) EcoHIV

An alternative approach to studying the effects of HIV was to modify the virus rather than the murine hosts. Previous studies which used human HIV co-receptors in mice failed to have efficient viral binding to either CCR5 or CXCR4 receptors and were poorly susceptible to HIV infection[235]. In the EcoHIV chimera virus, the gp120 coding region of HIV virus is replaced with the gp80 coding region of the murine leukemia virus [236]. This modified virus was selective to rodents and found to productively infect peripheral murine lymphocytes. These studies; however, showed limited detection in the brain following intraperitoneal injection of virus. Separately, Jones et al showed that as early as twoweeks post infection, EcoHIV was able to alter BBB permeability [237]. Despite this apparent discrepancy, direct administration of virus into the brain by intracranial injection is a viable means of using EcoHIV to study the neurological effects of HIV and show robust HIV expression [238]. 
(b) Viral protein injection

In favor of determining the roles of individual viral proteins, direct injections of viral proteins into mice have been widely utilized [199, 239-241]. Direct injection of viral proteins into the CNS allows for uniform exposure without potential confounders such as the degree of doxycycline induction and effects associated with individual viral proteins. Neurotoxic responses following injection is rapid while also available to test targets which may ameliorate or exacerbate effects. For example, numerous studies have used direct injection of HIV Tat in conjunction with drugs of abuse to survey concurrent effects [241244]. Alternatively, parallel or subsequent exposure to potential therapeutic agents and whether they can ameliorate neurodegenerative effects are an additional benefit of the direct injection models.

\subsection{Potential applications and conclusions}

As investigation occurs into the mechanisms of HIV and opioid-induced neurodegeneration, the models for study have also expanded. In terms of studying the autophagy pathway, there are numerous means of examining its role in mediating HAND using the plentiful rodent models available. For example, to study how the autophagy pathway functions to mediate the direct effects of HIV/viral proteins, there are a number of autophagy-deficient mouse models available which can be combined with the direct injection method of HAND induction (Figure 1.8a) [136, 158, 184, 245]. This method of study allows for acute study of effects in the context of an autophagy deficient environment. The various existing autophagy models have mutations in varying stages of the pathway and can thus be used to determine the relative temporal importance in the autophagy/HIV interaction. A potential alternative approach would be to utilize both an autophagy-deficient mouse model and a viral protein/HIV transgenic mouse to create a 
chimera which could have viral protein expression induced in an autophagy-deficient background (Figure 1.8b). Both models are capable of being used in conjunction with exposure to opioids by either subcutaneous morphine pellet or intraperitoneal injection for the study of HAND in the context of opioid abuse. The autophagy pathway poses an appealing target for ameliorating the effects of HAND in the opioid-abusing HIV-infected population. In addition, the humanized mouse models allow for the examination of potential therapeutics targeting the autophagy pathway with pharmacological inducers and inhibitors and the evaluation translational relevance which are the ultimate goal of these studies. The next steps in the field to determine how autophagy can be modulated to ameliorate the symptoms characteristic of HAND may lie in the pharmacological inhibition of specific stages in the pathway. For example, treatment of Tat-transgenic mice with the autophagy inducer rapamycin was able to reverse autophagosome accumulation and neurodegeneration [191]. Other avenues of treatment could include the use of siRNA

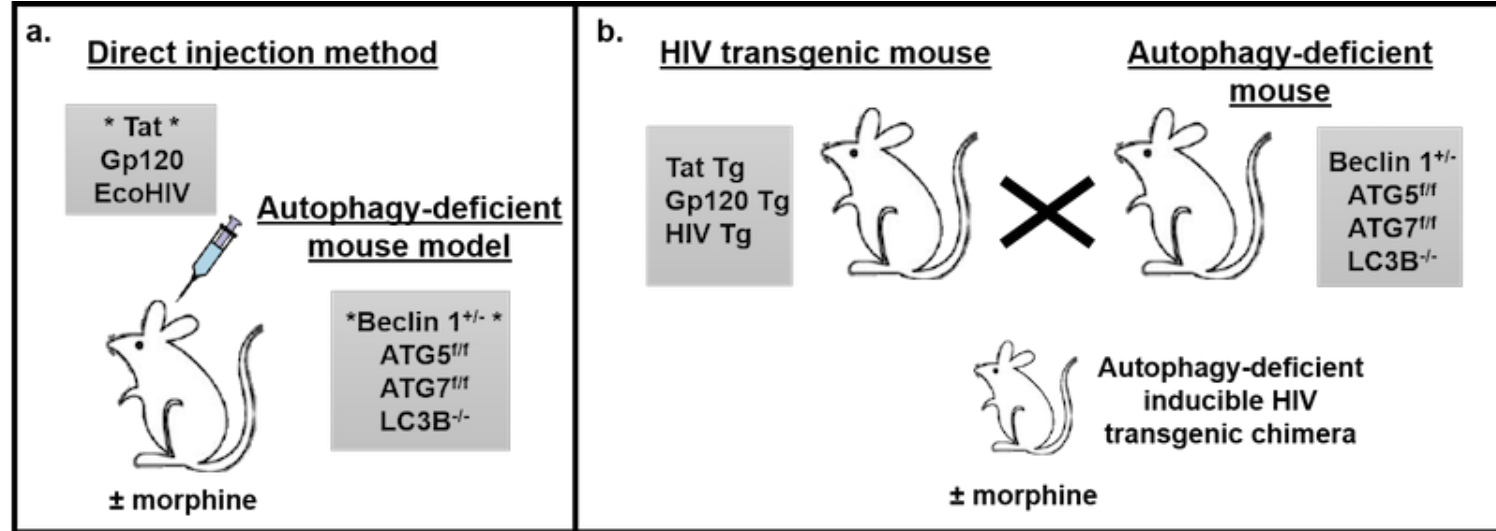

Figure 1.8 Various mouse models which can be used to study autophagy in HAND pathogenesis

(A.) Direct injection of neurotoxic viral proteins such as Tat or gp120 or the mouse-permissive chimera virus EcoHIV can be utilized to assess autophagy proteins which may contribute to neuropathogenesis. The stars $\left(^{*}\right)$ indicate the models used here with the viral protein Tat and the Beclin 1+/- mouse model. (B.) An alternate method to study autophagy protein contributions to HIV pathogenesis may generated by crossing inducible HIV-1 protein transgenic mice with autophagyprotein-deficient mice creating a chimera. Both models can be used in combination with morphine co-exposure. 
targeted towards autophagy proteins. Silencing of Beclin 1 in microglia and astrocytes was able to reduce inflammation as well as viral replication, making an attractive target for therapy $[192,246]$. Based on this, the use of siRNA targeting Beclin 1 has been coupled to 1.) the guided drug delivery system of magneto-electric nanoparticles (MENPs), where is was able to cross the blood brain barrier without toxic effects [247] and 2.) a biodegradable linear polymer cationic polyethylenimine (PEI) complex for intranasal delivery to the brain. The realm of HAND and the mechanisms determining its pathogenesis are still not clear; however, the autophagy pathway is an up and coming facet showing great promise for future innovation. Furthermore, the established background provides the groundwork for the aims of this dissertation and advancing the understanding of HIV and opiate-induced neuropathogenesis.

\subsection{Dissertation hypothesis and specific aims}

Based on the existing literature presented in the previous chapter, HIV and opiate-induced neuropathogenesis is a relevant and significant burden. Our lab and others have shown the interactive effects of the HIV protein Tat and morphine in brain cells such as microglia and astrocytes to facilitate neuroinflammation and oxidative stress $[79,106]$; however, the mechanisms at play are still widely unknown. Here we propose that the autophagy pathway, which is associated with HIV replication and persistence, may be a significant contributor to the virally-induced neuroinflammation and neuronal damage observed with HIV Tat and morphine co-exposure. In addition, previous in vitro data from our lab using human microglia treated with HIV-1 and morphine has shown induction of the autophagic pathway as well as accumulation of autophagosomes indicative of a halt in autophagic clearance [192]. When a key initiator of autophagy Beclin-1 was silenced using siRNA, there was a reduction of both HIV-1 replication in human microglia and astrocytes as well 
as in the HIV-1 induced inflammatory response. These data suggest that induction of the autophagy pathway may serve as a mechanism mediating the damaging interactive effects of HIV Tat and morphine within the brain and that targeting of this pathway can be utilized to attenuate these effects. Given these findings, we hypothesize that by reducing the expression of the autophagy protein Beclin 1, the neurodegenerative effects of Tat and morphine exposure can be ameliorated at both the cellular and animal level.

These hypotheses will be addressed in the experiments of the following Specific Aims:

\section{Specific Aim 1: To confirm if Tat and opiate-induced inflammation in astrocytes and} microglia (glia) are mediated by the autophagy pathway

These experiments assessed various aspects of glial dysfunction including neuroinflammation, oxidative stress, and calcium accumulation as a function of Tat and morphine treatment to mixed glial cell cultures (microglia and astrocytes) obtained from the striatum of wild type C57BL/6J and Beclin $1^{+/-}\left(\right.$Becn $\left.^{+/-}\right)$deficient mice. These mutant mice are heterozygous for the Becn 1 allele and show expression compared to C57BL/6J littermates.

\section{Specific Aim 2: To examine the roles of autophagy in neuronal outcome upon Tat} and opiate treatment

Provided that neurons are the cell type ultimately affected by the disruption of homeostasis in the brain caused by HIV-1 infection, the cytoprotective or cytotoxic role of autophagy on neuronal damage, survival, and toxicity were gauged with respect to Tat and morphine exposure utilizing embryonically-derived neurons of $\mathrm{C} 57 \mathrm{BL} / 6 \mathrm{~J}$ and Becn1+/- mice. 
Specific Aim 3: To determine whether the in vitro observations seen in Specific Aims 1 and 2 translate to behavioral modifications and brain pathology in vivo seen in HIV-associated neurological impairment.

C57BL/6J and Becn1+/- mice were exposed to intra-striatal Tat injections alone or in combination with subcutaneous morphine pellet implantation to evaluate effects on motor coordination and brain histology.

We anticipate that this study will reveal what function, if any, the Beclin 1-dependent autophagy pathway plays in directly mediating the Tat and morphine neurodegenerative interactions within the murine brain which can then be modulated pharmacologically. 
CHAPTER 2: CRITICAL ROLE OF BECLIN 1 IN HIV TAT AND MORPHINE-INDUCED GLIAL DYSFUNCTION

Parts of this chapter appear in the Journal of Neuroimmune Pharmacology. 2018 May 11. doi: 10.1007/s11481-018-9788-3.

\subsection{Introduction}

HIV neuropathogenesis can be attributed to not only the propagation of infection in the brain, but also to viral proteins being secreted by infected cells that can also cause neurotoxicity. HIV Tat protein, responsible for regulating the initiation of HIV transcription and elongation, is released from HIV-infected cells and has been found to be neurotoxic independent of virus $[57,248]$. The release of Tat contributes to the microglial-derived release of reactive oxygen species (ROS), nitric oxide (NO), calcium overload, and initiation inflammatory cascades, all of which impede neuronal survival [7, 249-252]. Other proteins within the HIV genome including Nef, gp120, Rev, Vpr and Vif also show varying effects on the brain and influence neurotoxicity through means of oxidative stress, secretion of pro-inflammatory molecules, and induction of apoptosis $[7,56]$. Drugs of abuse such as opiates are known to increase risk of exposure to HIV infection and influence the progression to AIDS. IDU become highly susceptible to HIV not only because of shared contaminated needles but also due to opiate induced immune suppression [253]. Of HIV-infected patients that are IDU, a population show an accelerated progression of HIV-associated neural pathogenesis characterized by HIVE [86]. HIV viral proteins and drugs of abuse show similar mechanisms of injury such as proinflammatory response, oxidative stress, and induction of apoptosis [79]. As such, co-exposure to HIV and opiate metabolites such as morphine have been shown to enhance viral replication and exacerbate the detrimental effects of HIV/Tat in the infected brain and promote 
progression to AIDS dementia [102, 103]. Despite studies to understand how the progression of HIV-associated neuronal pathogenesis is exacerbated by opiates, the mechanism is still not fully understood.

Autophagy is a cellular process in which cytoplasmic contents such as organelles and proteins are encapsulated in the double-layered membrane called an autophagosome and delivered to the lysosome for degradation and macromolecule recycling [254]. Autophagy can be initiated as a pro-survival response by a variety of stimuli such as environmental and cellular stress such as nutrient starvation, accumulation of aggregated proteins, or for pathogen internalization and degradation in innate immune cells [108]. This is particularly key in the brain cells since many are post-mitotic and require autophagy for maintaining homeostasis. Moreover, dysregulation in the autophagy pathway contributes to the development of a number of neurodegenerative diseases such as AD and PD [255]. Patterns of neurodegeneration similar to these diseases during HIV infection have been observed and linked to defects in the autophagy pathway. Though generally cytoprotective, autophagy plays an intricate role in viral replication with respect to infected cell type and can be induced or inhibited in order to bolster virus production at certain stages of the viral life cycle $[23,256]$.

We have previously shown that siRNA mediated silencing of the BECN1 gene can significantly reduce HIV p24 levels and HIV and morphine-induced inflammatory cytokine and chemokine secretion from infected primary human microglia and astrocytes [192, 246, 257, 258]. Beclin 1, a component of the phosphatidylinositol 3-kinase nucleation complex, regulates the initiation stages of autophagy preceding the formation of the isolation membrane of the autophagosome. As such, Beclin 1 poses an attractive target for future 
therapeutic intervention and for gaining a better understanding of the interplay between HIV, autophagy, and drugs of abuse. In the present study, we use a mouse model possessing a monoallelic deletion of the mouse Becn1 gene as a potential therapeutic model to provide a direct link between the autophagy protein Beclin 1 and HIV Tat and their effects on glial dysfunction. The data also support the role of autophagy in mediating Tat and morphine-induced neuropathology.

\subsection{Materials and Methods}

\subsubsection{Animals}

C57BL/6J mice (stock \# 000664) and the Becn1 deficient mouse model (B6.129X1-

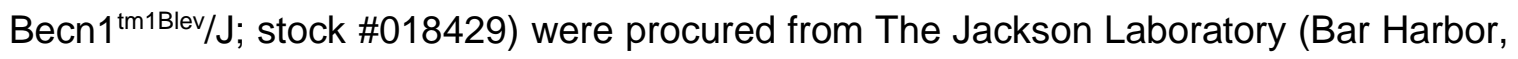
ME, USA) and bred in the animal facility at Florida International University. The Becn $1^{\text {tm1Blev }}$ mouse is heterozygous for the beclin 1 allele and was originally generated by Qu et al. [136]. Briefly, a targeting vector was designed to replace exons 1 and 2 with a neomycin resistance cassette. The construct was electroporated into 129X1/SvJ-derived embryonic stem cells with correctly targeted cells injected into blastocysts and the resulting chimeric mice were bred to C57BL/6J. Offspring were backcrossed to C57BL/6J for 50 generations. Homozygous deletion of the targeted allele results in embryonic lethality. C57BL/6J and Becn1 mutant mice were bred as necessary for experiments to minimize colony population with cross-bred mice exhibiting Mendelian inheritance genetics. Of note, mice on a C57BL/6J background have a brownish (agouti) coat color which is believed to be a result of the effect of the Becn1 mutation on melanogenesis (Figure 2.1a). The guidelines of the National Institutes of Health Guide for the Care and Use of Laboratory Animals were followed, and the Florida International University Institutional Animal Care and Use Committee approved all animal experimental protocols. 


\subsubsection{Mouse genotyping}

Genotyping of tail genomic DNA was performed to detect wild type and Becn1 knockout alleles by PCR amplification. The sense primer 5'-AGCCTCTGAAACTGGACACG-3'and the antisense primer 5'-TGGAAACAGGGTCTCATTCA-3' were used to detect the wild type beclin1 allele (yielding a PCR product of $380 \mathrm{bp}$ ), and the sense primer 5'CTCCAGACTGCCTTGGGAAAA-3' and the identical antisense primer were used to detect the knockout beclin1 allele (yielding a PCR product of approximately $400 \mathrm{bp}$ ). PCR was performed using AmpliTaq Gold® DNA Polymerase kit (Life Technologies) as per manufacturer's instructions and the PCR product identified by agarose gel electrophoresis.

\subsubsection{Mixed glial murine cell cultures}

For primary murine glial culture, post-natal day 4-6 Becn $1^{+/-}$mutant mice and C57BL/6J littermates were separated according to phenotypic coat color and sacrificed according to IACUC guidelines. Growth medium contained Dulbecco's Modified Eagle's Medium (DMEM; Invitrogen, Carlsbad, CA, USA) supplemented with glucose $(2 \mathrm{mg} / \mathrm{mL}$; SigmaAldrich), $\mathrm{Na}_{2} \mathrm{HCO}_{3}$ (6 mM; Invitrogen), 10\% v/v heat inactivated fetal bovine serum (FBS; Hyclone, Logan, UT, USA), and 1\% penicillin/streptomycin (100 U/mL / $100 \mu \mathrm{g} / \mathrm{mL}$; Invitrogen) [101]. Striata were isolated and dissociated mechanically and enzymatically with $0.25 \%$ trypsin containing DNase $(2.5 \mathrm{mg} / \mathrm{ml})$ centrifuged, triturated, and twice filtered through $40 \mu \mathrm{M}$ nylon mesh cell strainer. Cells were then plated and maintained in plates

coated with poly-L-lysine $(0.1 \mathrm{mg} / \mathrm{ml})$. Cultures were maintained for $5-10$ days at $37^{\circ} \mathrm{C}$ and $5 \% \mathrm{CO}_{2}$. 


\subsubsection{Immunocytochemistry}

4-well chambers containing adherent cells were washed with PBS, fixed in $4 \%$ paraformaldehyde, permeabilized with $0.1 \%$ Triton X-100, blocked in $0.1 \%$ Triton X-100 with $1 \%$ milk/1\% goat serum and immunolabeled. Primary antibodies anti-GFAP (catalog \# MAB360, Millipore) and anti-Iba1 (catalog \# 019-19741, Wako Chemicals) were each used at 1:400 and 1:50 dilutions for astrocyte and microglia visualization respectively. Immunoreactivity was visualized with secondary antibodies from Molecular Probes (Carlsbad, CA, USA). Cells were mounted with ProLong® Gold antifade reagent with DAPI (Thermo Fisher Scientific). Images were analyzed using a Zeiss (Germany) inverted fluorescence microscope with a 560 Axiovision camera.

\subsubsection{Western blotting}

Whole cell lysates were prepared in RIPA buffer supplemented with a mixture of protease and phosphatase inhibitors and separated by SDS-PAGE for western blotting. Primary antibodies: Beclin 1 - 1:500 (Novus Biologicals, NB500-249), LC3A/B - 1:1000 (Cell Signaling, 12741S), P62/SQSTM1 - 1:500 (Novus Biologicals, NBP1-48320), LAMP1 1:500 (Novus Biologicals, NB120-19294), $\beta$-actin - 1:200 (Santa Cruz, sc-47778). Following primary antibodies, blots were incubated with horseradish peroxidaseconjugated secondary antibodies (Cell Signaling Technology, 7076S; Sigma-Aldrich, A0545) used at a 1:1000 dilution. Immunoblots were exposed to SuperSignal West Femto Substrate (Thermo Fisher Scientific) and visualized using a ChemiDoc imaging system (Bio-Rad, Hercules, California). Protein expression was calculated using Image Lab software (Bio-Rad). 


\subsubsection{LC3 Autophagy Sensor}

Levels of autophagy in primary glia derived from C57BL/6J and Becn1 ${ }^{+/}$mice were assessed using the Premo ${ }^{\mathrm{TM}}$ Autophagy Sensor LC3B-GFP kit (Thermo Fisher Scientific, Grant Island, NY, USA) following manufacturer's instructions. Cells were treated with 10 $\mu \mathrm{L}$ of BacMam reagent containing the LC3B-GFP construct for 16 hours. Rapamycin (Sigma-Aldrich, St. Louis, MO, USA) was used at $2.5 \mu \mathrm{M}$ as an autophagy inducer of autophagy. After incubation, cells were washed with PBS and mounted with ProLong® Gold anti-fade reagent with DAPI (Thermo Fisher Scientific). Images were analyzed for green fluorescence (LC3B positive autophagosomes) using a Zeiss (Germany) inverted fluorescence microscope with a 560 Axiovision camera

\subsubsection{Reagents and treatments}

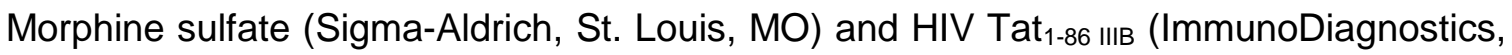
Woburn, MA) were used at concentrations ranging from $100 \mathrm{nM}-1 \mu \mathrm{M}$ and $10 \mathrm{nM}-100$ $\mathrm{nM}$ respectively. The HIV Tat ${ }_{1-86}$ protein is derived from the CXCR4 tropic HIV-1 IIIB strain which was expressed in the E.coli expression system as a recombinant protein. Furthermore, it has been previously shown that the neurotoxic domain of Tat is well conserved across various strains of HIV [259]. This concentration of Tat has been shown to not only induce inflammatory cytokine production and other homeostatic dysregulations in glial cells in vitro, but also causes toxicity to neurons. Given that treatments of 100 $\mathrm{ng} / \mathrm{mL}$ and $1000 \mathrm{ng} / \mathrm{mL}$ correspond to approximately $8.3 \mathrm{nM}$ and $83.3 \mathrm{nM}$ concentrations respectively, we deemed the concentration range of $10 \mathrm{nM}$ to $100 \mathrm{nM}$ acceptable. It may also worth noting that both the Kruman and Nath studies utilize the HIV BRU Tat ${ }_{1-72}$ which may account for differences in extent of effects. In addition, this lab has used these

concentrations in previous studies of Tat induced neuroinflammation [66, 102, 260, 261]. 
Morphine at a concentration of $500 \mathrm{nM}$ has been previously shown to fully activate MOR as well as enhance HIV Tat mediated neurotoxicity [101, 261]. $\mu$-opioid receptor antagonist naltrexone (Sigma-Aldrich, St. Louis, MO) was used at a concentration of 500 $\mathrm{nM}$ and was added 30 minutes prior to morphine exposure. Viral proteins Nef, Rev, and Gag were used at 1 and $5 \mathrm{nM}$ envelope protein gp120 was used at 200 and $500 \mathrm{pM}$ (NIH AIDS reagent program).

\subsubsection{ELISA}

Mouse glial cell culture supernatants were used to measure the levels of cytokines and chemokines, which have been shown to be released upon HIV Tat treatment [261]. These included interleukin (IL) -6, monocyte chemotactic protein-1 (MCP-1), regulated upon activation normal T-cell expressed and secreted (RANTES), and tumor necrosis factor alpha (TNF- $\alpha$ ) which were assayed by ELISA (R\&D Systems, Minneapolis, MN) according to the manufacturer's instructions. The optical density was read at $A_{450}$ with wavelength correction at $A_{570}$ on a Synergy HTX plate reader (Biotek, Winooski, VT)

\subsubsection{Quantitative Reverse Transcription-Polymerase Chain Reaction}

Total RNA was isolated from glia following 24-hour treatments using the miRNeasy Mini Kit (Qiagen, Valencia, CA). Purity was assessed by microspot RNA reader (Synergy HT Multi-Mode Microplate Reader from BioTek) and RNA preparations with an OD260 $\mathrm{nm} / \mathrm{OD} 280 \mathrm{~nm}$ absorbance ratio of at least 2.0 were used for cDNA synthesis. $1 \mu \mathrm{g}$ of RNA was used for cDNA synthesis using High-Capacity cDNA Reverse Transcription Kit (Applied Biosystems; Cat \#: 4368814) as per the manufacturer's protocol. Relative abundance of mRNA was assessed by SsoAdvanced Universal SYBR Green Supermix (BioRad, Cat \#: 172-5271) in $20 \mu \mathrm{L}$ real-time PCR reactions with gene specific primers 
using a BioRad CFX96 real time system. All data were normalized to $\beta$-actin mRNA and presented as $2^{\wedge-\Delta \Delta C t}$ for fold-change values. Primers used for amplification were as follows: mouse $\quad$ 5'-TGGAAACTTCCTGGTCATGT-3' and 5'AGATCACGATCTTGCAGAGG-3' (182bp); mouse DOR, 5'GAGGATAAGTGGGGGATGGT-3' and 5'-AGCCTCAGCCTCCACTATGA-3' (175bp); mouse KOR, 5'-CCAGCATATTCACCTTGACC-3' and 5'GAAGAGATCCCACCAGGAAT-3' and mouse $\beta$-actin, 5'AAGAGCTATGAGCTGCCTGA-3' and 5'-TACGGATGTCAACGTCACAC-3' (160bp).

\subsubsection{Cell viability}

Toxicity of HIV Tat and morphine in mixed glial cultures was assessed using 12 mM MTT (3-(4, 5-Dimethylthiazol-2-yl)-2, 5-Diphenyltetrazolium Bromide) for cell survival and proliferation (Millipore, Temecula, CA). Cells were plated in poly-L-lysine coated 96 well plates at a density of $3 \times 10^{4}$ cells/well from T75 flasks and cultured overnight followed by treatment with $100 \mathrm{nM} \mathrm{HIV} \mathrm{Tat} \mathrm{and} \mathrm{increasing} \mathrm{concentrations} \mathrm{of} \mathrm{morphine} \mathrm{ranging} \mathrm{from}$ $100 \mathrm{nM}$ to $1 \mu \mathrm{M}$. After 24 hours, cells were refed with $100 \mu \mathrm{L}$ fresh media and $10 \mu \mathrm{L}$ of 12 mM MTT was added to each well except for control and incubated for 2 hours to allow the formation of formazan crystals. Media was removed from wells and formazan crystals dissolved using DMSO. Absorbance was read at $570 \mathrm{~nm}$ with reference at $630 \mathrm{~nm}$ on a Synergy HTX plate reader (Biotek, Winooski, VT).

\subsubsection{Reactive oxygen species (ROS)}

Levels of intracellular ROS production were measured using dichlorodihydrofluorescein diacetate $\left(\mathrm{CM}-\mathrm{H}_{2}\right.$ DCFDA, Invitrogen, Carlsbad, $\left.\mathrm{CA}\right)$, which is de-acetylated to dichlorofluorescein (DCF). Mixed glial cells were incubated with $10 \mu \mathrm{M} \mathrm{CM}-\mathrm{H}_{2}$ DCFDA in 
warm PBS for 1 hour according to the manufacturer's protocol, washed then treated. Nacetylcysteine, a precursor to the antioxidant glutathione, was used as a control at $10 \mu \mathrm{M}$ and pre-treated for 1 hour. $\mathrm{H}_{2} \mathrm{O}_{2}(0.001 \% \mathrm{v} / \mathrm{v})$ was added at time of treatment as a positive indicator of oxyradical species. Dichlorofluorescein (DCF) fluorescence was measured at an excitation wavelength $\left(\lambda_{\mathrm{ex}}\right)$ of $485 \mathrm{~nm}$ and an emission wavelength $\left(\lambda_{\mathrm{em}}\right)$ of $520 \mathrm{~nm}$ over 24 hours using a Synergy HTX plate reader (Biotek, Winooski, VT). Relative DCF fluorescence estimated the levels of ROS present and were reported as DCF mean fluorescence intensity (MFI).

\subsubsection{Nitric oxide (NO) production}

Measurement of NO was assessed from the supernatant of control and HIV Tat \pm morphine treated cells after $0,1,6$, and 24 hours. Collected supernatant was measured for the concentration of nitrite (the oxidized metabolite of NO) and evaluated using a nitric oxide assay kit (BioVision, Milpitas, CA) according to manufacturer's instructions. Absorbance was measured at $540 \mathrm{~nm}$ using a Synergy HTX plate reader (Biotek, Winooski, VT). The concentration of NO in samples was calculated based on the standard curve using known concentrations of nitrite.

\subsubsection{Intracellular calcium $\left[\mathrm{Ca}^{2+}\right]_{\mathrm{i}}$ measurements}

Levels of $\left[\mathrm{Ca}^{2+}\right]_{i}$ in glial cultures were measured using the fluorescent marker Fura-2-AM (Invitrogen, Carlsbad, CA). Cells were loaded with $5 \mu \mathrm{M}$ Fura-2-AM for 30 minutes at $37^{\circ} \mathrm{C}$, $5 \% \mathrm{CO} 2$. After three washes, the cells were incubated in growth medium for an additional 30 minutes to complete de-esterification of the AM group of the fluorophores. Cells were pre-treated with $10 \mu \mathrm{M}$ of the cell permeant calcium chelator BAPTA-AM (Tocris Bioscience) as a negative control prior to addition of Fura-2-AM. Baseline measurements 
were recorded, followed by exposure to Tat and/or morphine. Fura-2 ratio at 340/380 nm excitation measurements were taken every 10 seconds for 30 minutes. Data is presented as percent of control values \pm SEM from a 3 separate experiments (15-25 cells).

\subsubsection{Statistical analysis}

Results are reported as mean \pm SEM of 3-6 independent experiments. Data were analyzed using analysis of variance (ANOVA) techniques, followed by Tukey's post hoc test for multiple comparisons (GraphPad Prism 7 software, La Jolla, CA). A value of $p<$ 0.05 was considered significant.

\section{$\underline{2.3 \text { Results }}$}

\subsubsection{Molecular characterization of Beclin 1 using primary glial cell cultures derived from C57BL/6J and autophagy deficient mouse}

Beclin 1-mediated autophagy is of key importance in maintaining brain homeostasis, with dual deletion of the Becn1 allele $\left(B e c n 1^{-/-}\right.$) being embryonically lethal and conditional knockout causing neurodegeneration in mice $[137,262]$. In order to study the role of host autophagy in the context of HIV viral protein effects, we instead make use of a monoallelic deletion mutant of Beclin 1 (Becn1 $\left.{ }^{+/}\right)$. B6.129X1-Becn1tm1Blev/J mice are heterozygous for the Becn1 allele $\left(B e c n 1^{+/}\right.$) and have a brown (agouti) coat color which is believed to be a result of the effect of the Becn1 mutation on melanogenesis and can thus be distinguished from wild type littermates phenotypically. This study makes use of primary glia derived from $\mathrm{C} 57 \mathrm{BL} / 6 \mathrm{~J}$ and Becn1 ${ }^{+/}$pups according to defined breeding scheme (Figure 2.1a). We first characterized Beclin 1 expression within the primary mixed glia derived from the mutant Becn1 ${ }^{+/-}$mice. Mice initially separated by coat color 4-6 days after birth were genotyped to confirm the BECN1 single allele mutation using DNA isolated from 
mouse tails and amplified by PCR. Amplification of the wild type sequence (14946) yielded a PCR product of 380bp in both black and agouti pups as expected, whereas the mutant primer (olMR8619) yields a product of $400 \mathrm{bp}$ only in agouti pups, confirming the mutation (Figure 2.1b,c). Knockdown of Beclin 1 protein in murine mixed glia was confirmed by western blot analysis and indicated a $60 \%$ reduction in expression levels (Figure $2.1 \mathrm{~d}$ ).

a.

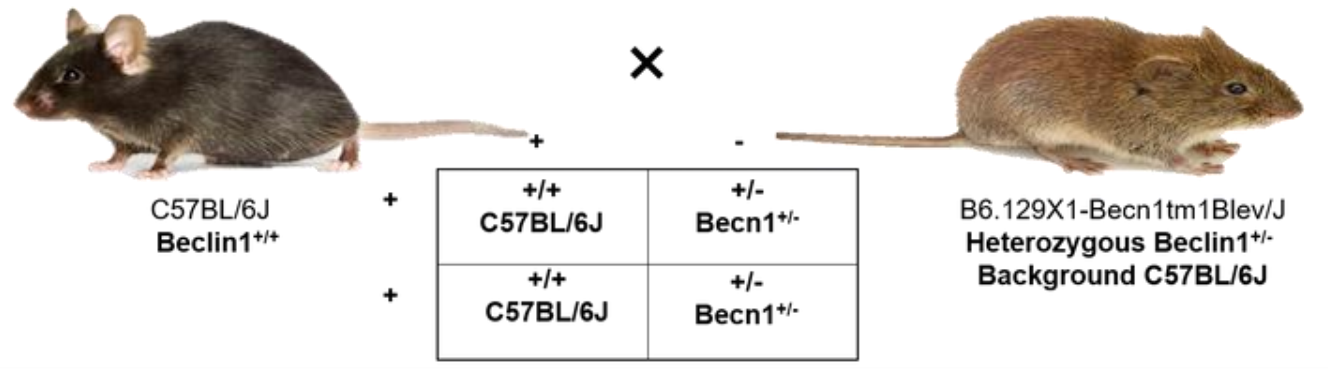

b.

\begin{tabular}{lll}
\hline 14946 & AGC CTC TGAAAC TGG ACA CG & Wild type Forward \\
14947 & TGG AAA CAG GGT CTC ATT CA & Common (Reverse) \\
olMR8619 & CTC CAG ACT GCC TTG GGAAAA & Mutant Forward \\
\hline
\end{tabular}

c.

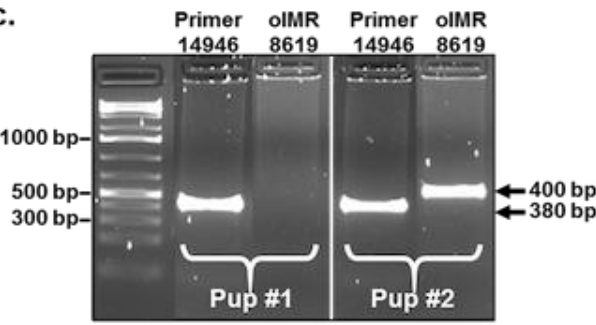

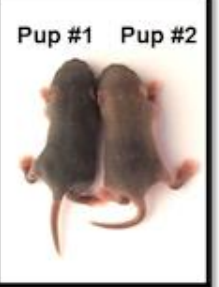

d.

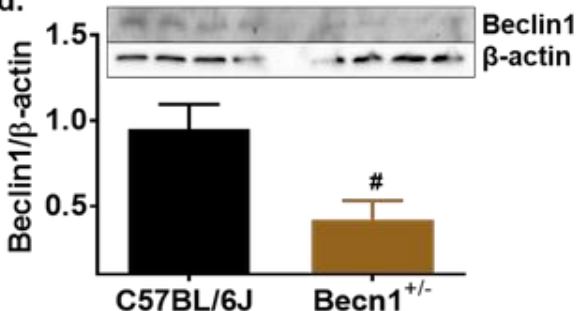

Figure 2.1 Characterization of Beclin 1-deficient mixed glia

C57BL/6J (Beclin $1^{++}$) and Becn1 ${ }^{+/}$mutant breeding scheme (a). Primers corresponding to the wild type and mutant beclin1 DNA sequence used for genotyping (b). Pups at P4-P6 were distinguished by coat color and mutation of the beclin 1 gene was confirmed at the DNA and protein levels by PCR genotyping (c) and western blot (d). Lanes are grouped according to sample number (pup \#1 vs pup \#2) and labeled according to the primer sequence used for amplification. Wild type sequence generates a PCR product of $380 \mathrm{bp}$ and mutant sequence generates a PCR product of $400 \mathrm{bp}$ (indicated by black arrows). Beclin 1 protein expression from isolated primary glia was normalized to $\beta$-actin. Data is presented as the mean \pm SEM of 4 mouse pups per strain. $P<0.05$ \# vs. C57BL/6J

Before continuing with the study, it was important to ascertain whether the Beclin 1 deficient glial cells could induce autophagy and to what extent the mutation reduced this 
capability. To determine whether a single allele mutation in the BECN1 gene is sufficient to considerably impair the autophagy pathway, we compared induction of autophagy in C57BL/6J and Becn1+/- derived glia using a fluorescent autophagy marker LC3-GFP assay. Upon induction of autophagy, LC3 localizes to pre-autophagosomal and

Control
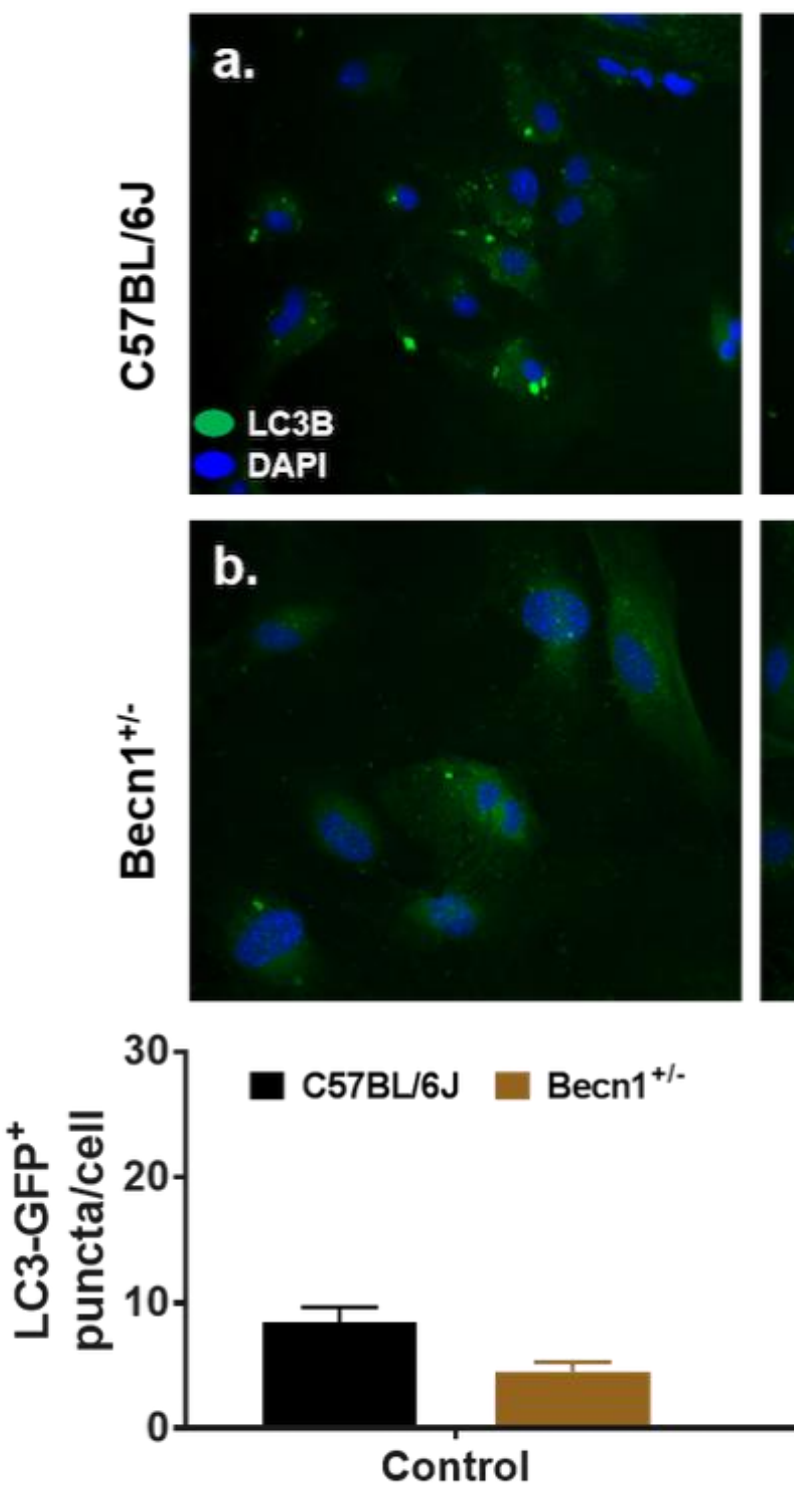

Rapamycin
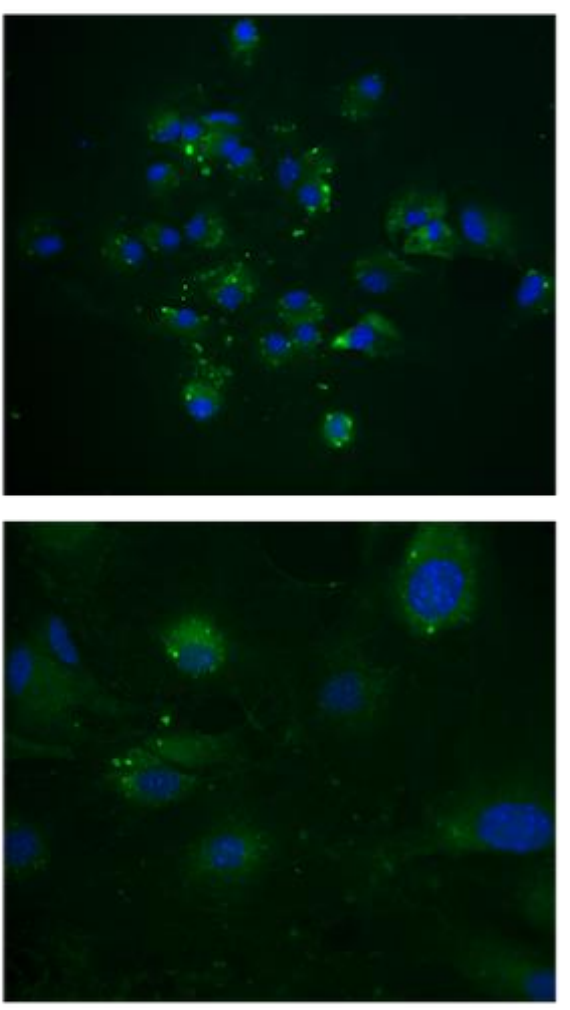

\#

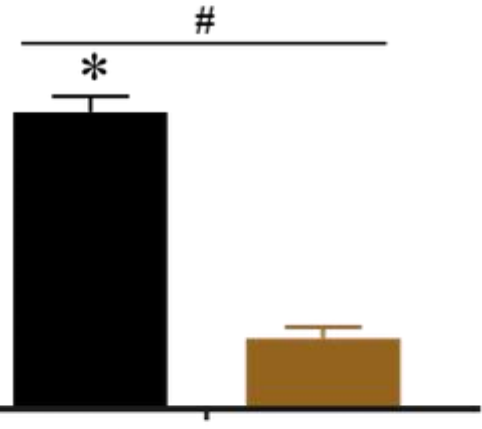

Rapamycin

Figure 2.2 Autophagy induction in C57BL/6J and Becn1 ${ }^{+/-}$glia

Representative images assessing basal autophagy induction by LC3-GFP puncta quantification upon treatment with the autophagy inducer rapamycin. Data was quantified from 2 independent experiments, averaging puncta per cell for 5 fields of view presented as mean \pm SEM. $P<0.05$ * vs. Control; \# vs. C57BL/6J. Two-way ANOVA followed by Tukey's test. 
autophagosomal membranes which can be indicated by the punctate appearance of GFP staining [263]. We observed that untreated Becn1 ${ }^{+/-}$glia show a slight but insignificant decrease in LC3-GFP puncta as compared to untreated C57BL/6J glia (Figure 2.2); however, when treated with the mTOR inhibitor and autophagy inducer rapamycin, C57BL/6J glia showed a 64\% increase in LC3-GFP puncta whereas there was no corresponding increase in Becn1 ${ }^{+/}$glia. These findings further demonstrate that a $60 \%$ reduction in protein levels due to the heterozygous deletion of the Becn1 allele correlates to a reduction of autophagic induction in glial cells upon autophagy induction. Morphology and glial cell components were also assessed by immunofluorescent labeling with the astrocyte marker, GFAP, and the microglia marker, Iba1 (Figure 2.3).
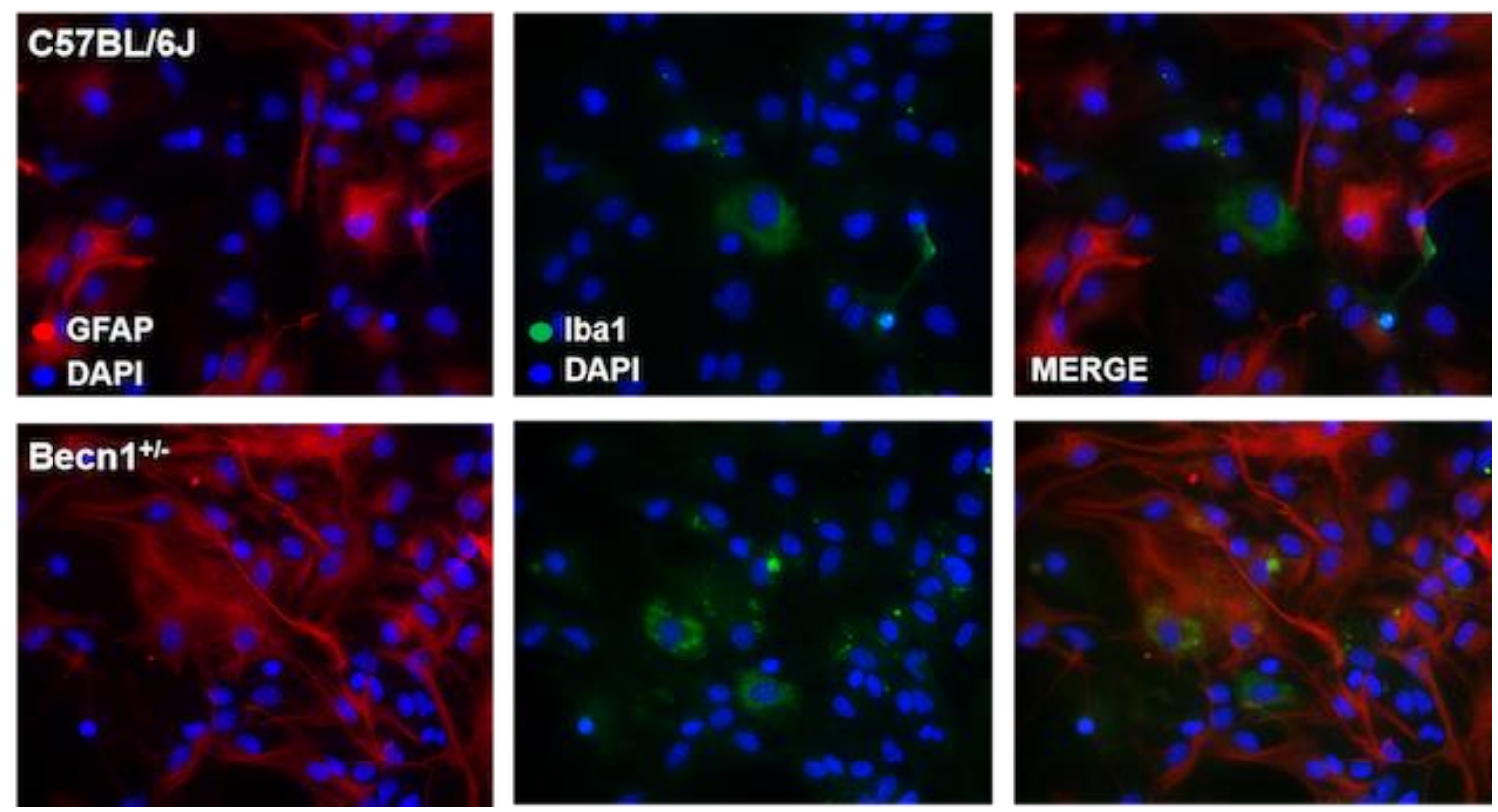

Figure 2.3 Immunocytochemistry for glial cell presence identifying $\mathrm{GFAP}^{+}$astrocytes in red and $\mathrm{Iba} 1^{+}$microglia in green

Primary glial cultures were immunolabeled to assess cell population and morphology. Red: GFAP+ astrocytes, Green: Iba1+ microglia, Blue: DAPI stained nuclei. 


\subsubsection{Beclin $1^{+/}$glia show reduced inflammatory molecule induction upon exposure to the viral protein Tat}

We have recently shown that HIV replication and viral-induced neuroinflammation are regulated via a Beclin 1-dependent pathway [192, 257, 258]. Given the established role of Tat as a mediator of neuroinflammation, we sought to determine whether the underlying mechanism is through Beclin 1. Mouse models, including the use of glial cells, have been widely used in the studies of HIV-induced neurotoxicity. While true that murine cells are restricted for HIV infection, exposure to viral proteins such as Tat, Nef, and gp120 elicit glial cell activation, inflammatory molecule secretion, macrophage infiltration into the brain, metabolic dysfunction, and signaling pathway alterations which are also seen in primary human glia, despite lacking infectious capability, and reflect levels seen pathophysiologically [65, 199, 240, 261, 264]. Glial cells derived from C57BL/6J and Beclin $1\left(B e c n 1^{+-}\right)$deficient mice were treated with increasing concentrations of the viral protein Tat for 8 and 24 hours for assessment of cytokine and chemokine secretion by ELISA (Figure 2.4). After 8 hours post-treatment, Tat caused a significant concentrationdependent increase in the release of RANTES, MCP-1, and IL-6 in C57BL/6J glia where Becn1+/- glia showed significantly less secretion by comparison (Figure 2.4; red, blue, and green arrows). These decreases were maintained at 24 hours for RANTES and IL-6 secretion. At $100 \mathrm{nM}$ Tat treatment, RANTES, MCP-1, and IL-6 secretion in Becn1+/ glia were reduced by 1.6, 2.7, and 1.3-fold at 8 hours, respectively; and 2.2, 1.3, and 1.2-fold 

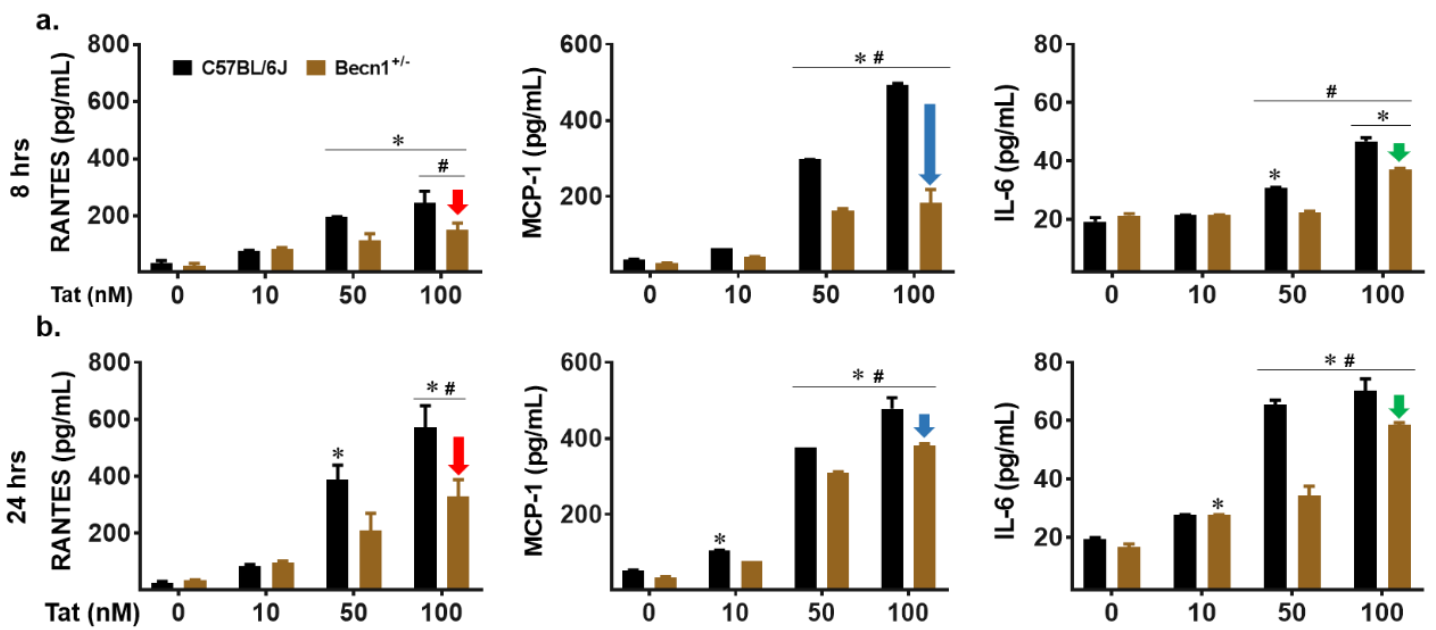

Figure 2.4 Beclin 1 facilitates Tat-induced inflammatory molecule secretion

Indicated cytokines and chemokines secreted from C57BL/6J and Becn $1^{+/-}$mixed glia supernatants were measured by ELISA at 8 (a) and 24 hours (b) following increasing concentrations of HIV Tat $(10 \mathrm{nM}, 50 \mathrm{nM}, 100 \mathrm{nM})$. Arrows indicate decreased Tat-induced secretion of RANTES (red), MCP1 (blue), and IL-6 (green) from Becn $1^{+/}$derived glia. Values were determined from standard curves and are presented as the mean \pm SEM of 5 independent experiments. $\mathrm{P}<0.05{ }^{*}$ vs. Control; \# vs. C57BL/6. Two-way ANOVA followed by Tukey's test.

at 24 hours respectively. Secretion of the pro-inflammatory cytokine TNF- $\alpha$ was also assessed; however, no differences were seen between Tat treatments or glial strains (data not shown). From this data, it can be inferred that Tat utilizes Beclin 1 to induce inflammatory molecule secretion and that $60 \%$ reduction in Beclin 1 expression level, can decrease this cytokine and chemokine release. To confirm the effects of Tat on cytokine/chemokine secretion were dependent on active protein, Tat was heat inactivated for 30 minutes at $95^{\circ} \mathrm{C}$ before being treatment to $\mathrm{C} 57 \mathrm{BL} / 6 \mathrm{~J}$ and Becn1 ${ }^{+/-}$glia and incubated for 24 hours (Figure 2.5). Secretion of RANTES was reduced by 7-fold (Figure 2.5a), and 3-fold in MCP-1 and IL-6 (Figure 2.5b,c) from glia treated with heat inactivated Tat illustrating the specific effects of active Tat on wild type and Beclin 1 mutant murine glia. Inflammatory effects of additional viral proteins were also surveyed though secretion was either less than that of Tat or showed no significant difference between the strains (Figure 2.6). Interestingly, the viral protein Nef also showed a significant degree of 

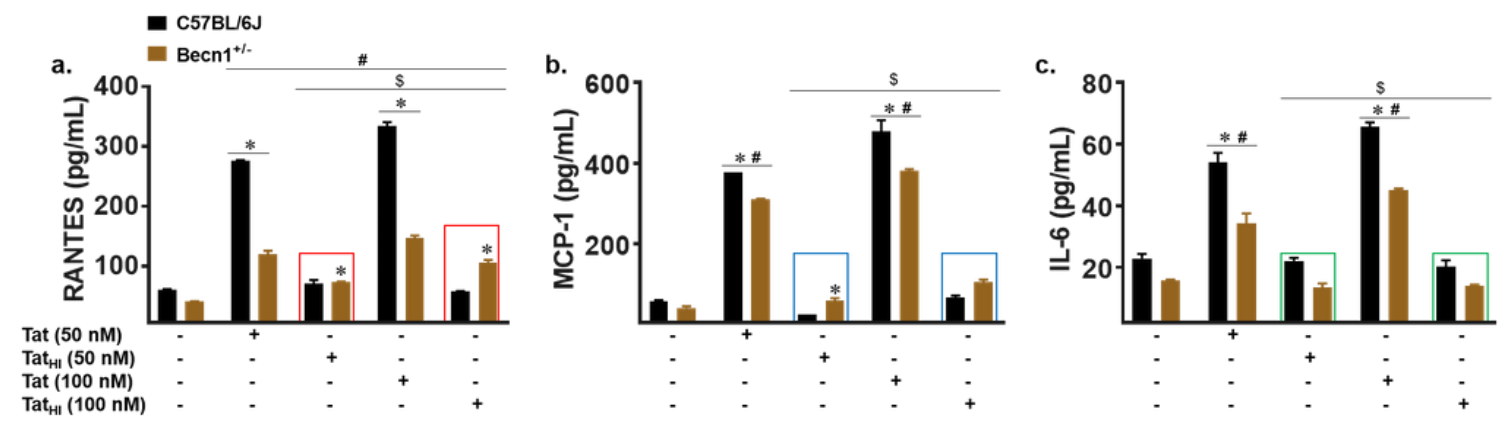

Figure 2.5 Inactive Tat is unable to interact with Beclin1 to induce cytokine secretion $\mathrm{C} 57 \mathrm{BL} / 6 \mathrm{~J}$ and Becn $1^{+-}$derived glia were treated with heat inactivated Tat $(50 \mathrm{nM}$ or $100 \mathrm{nM})$ for 24 hours and showed limited secretion of RANTES (a), MCP-1 (b), and IL-6 (c). Heat inactivated treatments are indicated in colored boxes. Values were determined from standard curves and are presented as the mean \pm SEM of 3 independent experiments. $\mathrm{P}<0.05{ }^{*}$ vs. Control; \# vs. C57BL/6J; \$ vs. Tat (50 nM). Two-way ANOVA followed by Tukey's test.

cytokine and chemokine induction from glia at low concentrations (1 $\mathrm{nM})$ and were nearly on par with that of Tat at a higher concentration $(5 \mathrm{nM})$ after 24 hours of treatment. In addition, whereas lack of Beclin 1 mediated autophagy was able to stem Tat-induced cytokine/chemokine secretion, treatment with Nef at high concentrations was able to override any ameliorating effects of the Becn $1^{+/-}$glia with the exception of MCP-1. This was further highlighted with the enhanced secretion of TNF- $\alpha$ at a high concentration of Nef treatment (Figure 2.6c,d; grey arrows). Overall, this supports the role of HIV Tat protein as a major facilitator of neuroinflammation in glia which can be regulated by the 
presence or absence of Beclin 1 while other viral proteins such as Nef play lesser but still significant factors potentially independent of Beclin 1 .

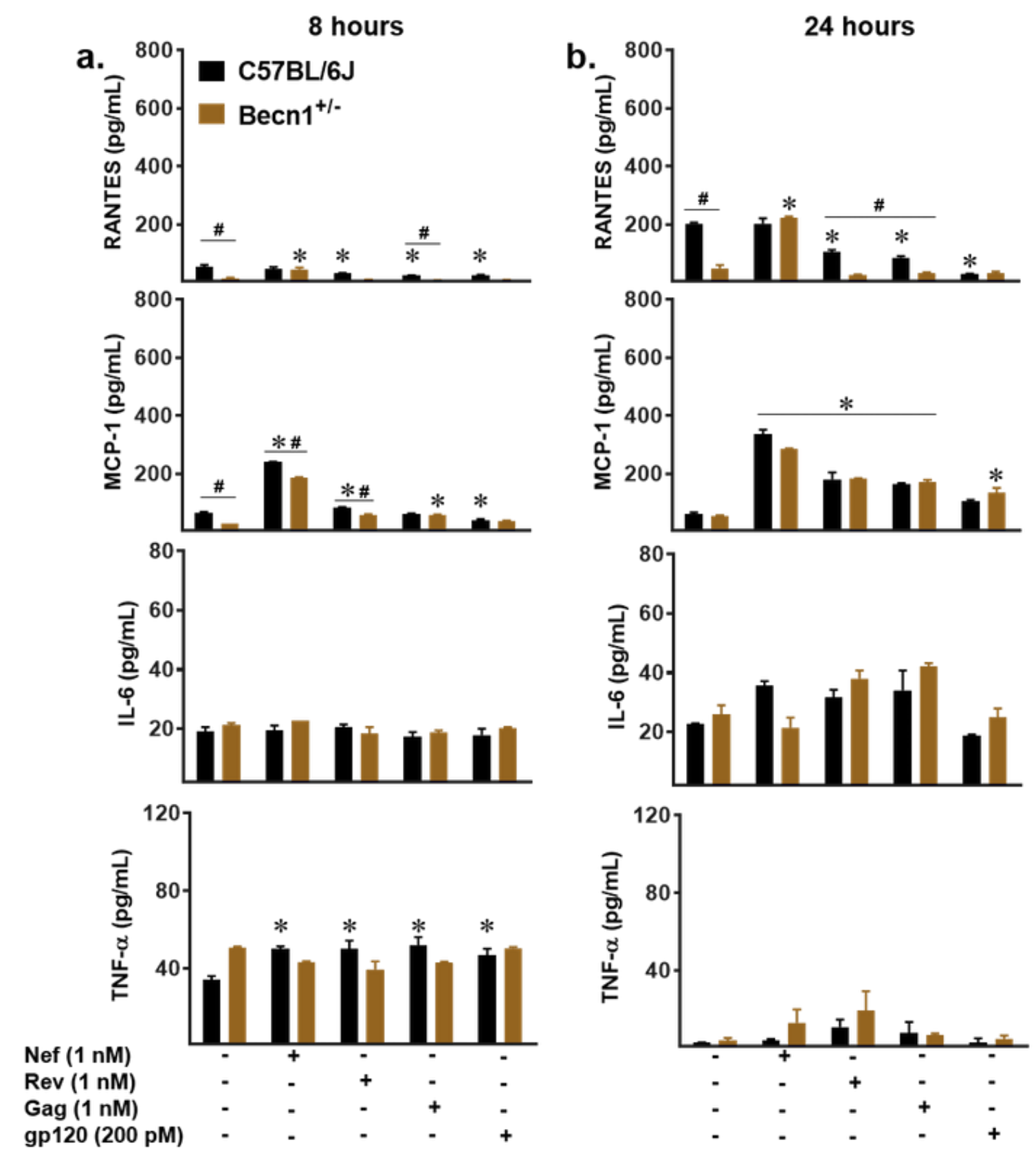



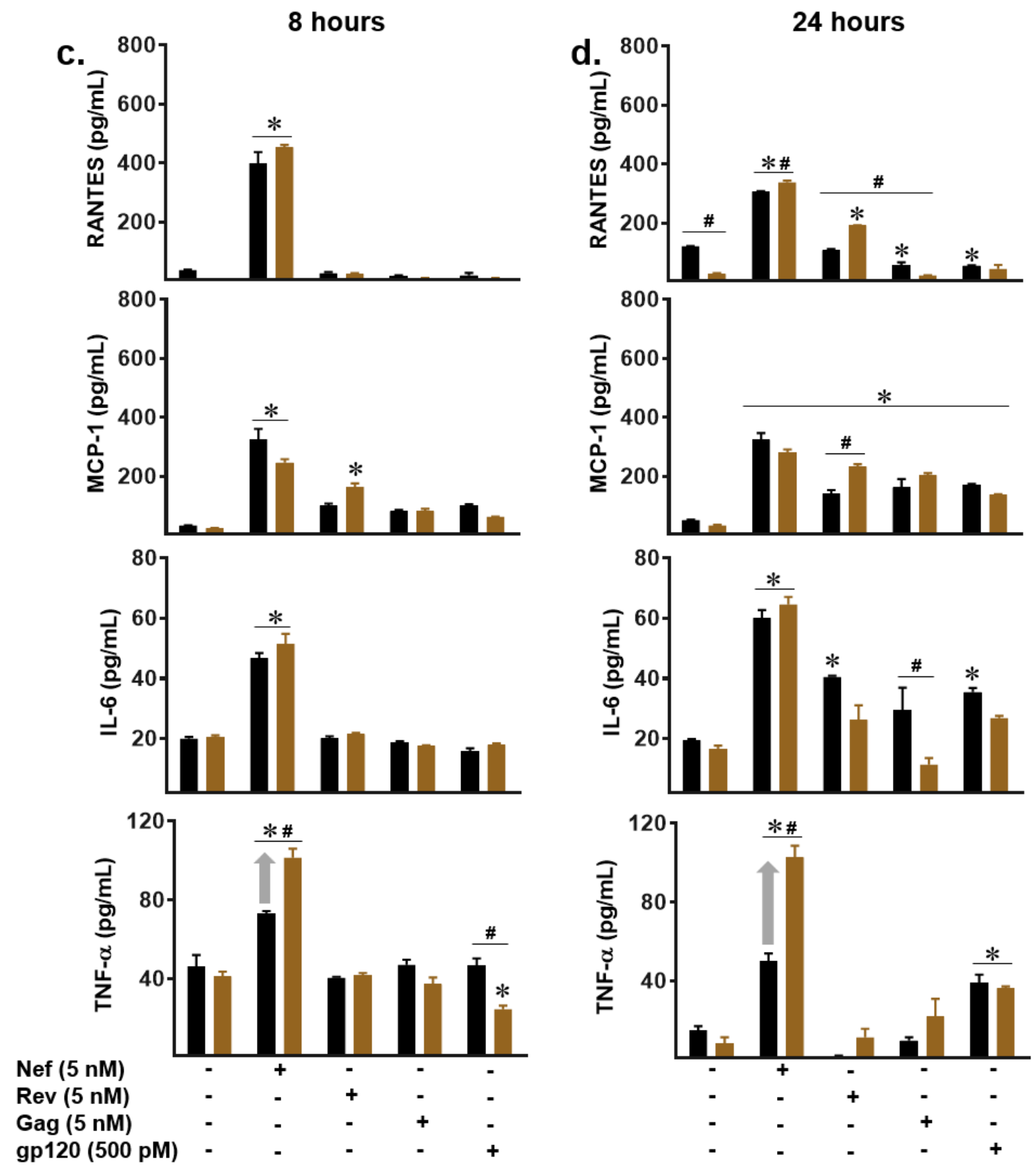

Figure 2.6 Effects of viral proteins on inflammatory molecule secretion through Beclin 1 Indicated cytokines and chemokines secreted from C57BL/6J and Becn $1^{+/-}$mixed glia supernatants were measured by ELISA at 8 ( $a$ \& c) and 24 hours (b \& d) following treatments of recombinant viral proteins. Nef (1 Nm/5 nM), Rev (1 nM/5 nM), Gag (1 nM/5 nM), gp120 (200 pM/500 pM). a: 8 hours, Nef (1 nM), Rev (1 nM), Gag (1 nM), gp120 (200 pM); b: 24 hours, Nef (1 nM), Rev (1 nM), Gag (1 nM), gp120 (200 pM); c: 8 hours, Nef (5 nM), Rev (5 nM), Gag (5 nM), gp120 (500 pM); d: 24 hours, Nef (5 nM), Rev (5 nM), Gag (5 nM), gp120 (500 pM). Values were determined from standard curves and are presented as the mean \pm SEM of 3 independent experiments. $P<0.05$ * vs. Control; \# vs. C57BL/6J. Two-way ANOVA followed by Tukey's test. 
Numerous studies have reported on the interplay between inflammation and autophagy, with induction of autophagy able to regulate inflammatory molecule secretion while conversely, cytokine secretion also being able to induce autophagy [265-267]. As such, we were interested in the effects of Tat on the expression of key autophagy proteins Beclin 1, LC3, and the ubiquitin-binding protein, p62/SQSTM1 since these proteins represent the initiation, autophagosome formation, and maturation stages of the autophagy pathway [79]. Correlating to the effects of Tat on cytokine secretion, there were concentration dependent changes in expression of autophagy related proteins Beclin 1 and p62/SQSTM1 in C57BL/6J-derived glia (Figure 2.7) suggesting induction of the autophagy pathway as well as accumulation of p62/SQSTM1 upon Tat treatment. Becn1+/- glia show reduced expression of Beclin 1 as expected; however, these levels were elevated with increasing Tat concentration. Interestingly, p62/SQSTM1 expression was unchanged with increasing concentration of Tat, though significantly less than C57BL/6J, which may suggest autophagic degradation is still ongoing, perhaps through Beclin 1-independent mechanisms. Taken together, this data indicates that the inflammatory molecule secretion induced by Tat is somewhat facilitated through an association with Beclin 1 and possibly the activation of the autophagy pathway.
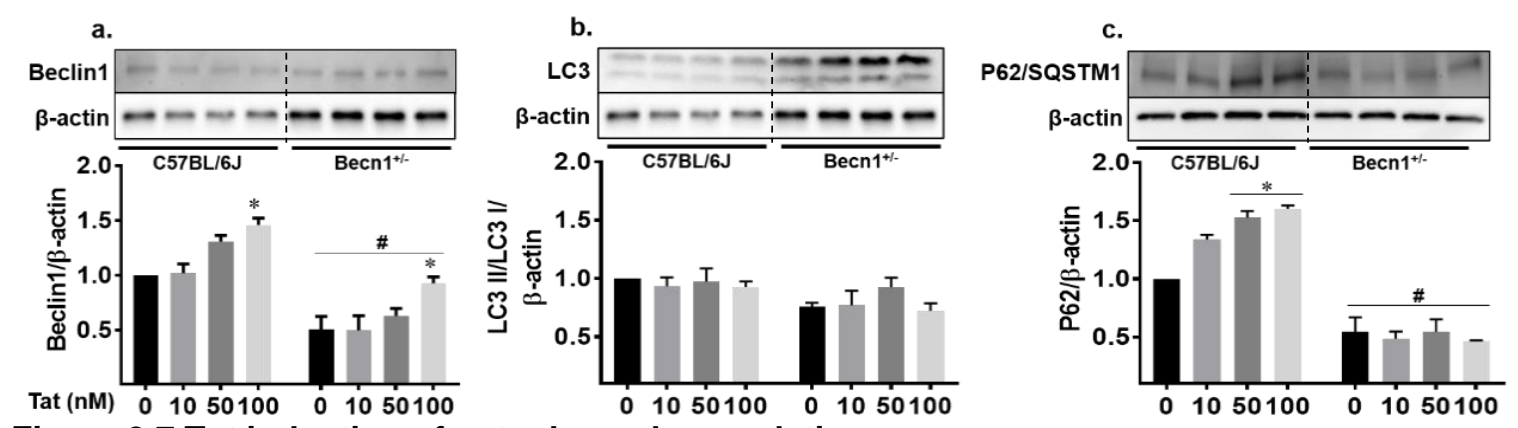

Figure 2.7 Tat induction of autophagy dysregulation

Whole cell lysates from C57BL/6J and Becn $1^{+/-}$glia after 24-hours of treatment were subjected to immunoblotting with antibodies to Beclin 1 (a), LC3 (b), and p62/SQSTM1 (c). Densitometry was performed for quantification, and the ratios of each protein to $\beta$-actin are presented graphically. Error bars show the SEM of 3 independent experiments. $P<0.05{ }^{*}$ vs. Control; \# vs. C57BL/6J. Two-way ANOVA followed by Tukey's test. 


\subsubsection{Beclin 1 mediates glial inflammation induced by Tat alone and in combination with morphine}

Given the co-morbidity of opiate abuse and HIV infection, we were next interested in the role of Beclin 1 on the effects of co-exposure to Tat and morphine on cytokine and chemokine secretion. First, we sought to determine the effect of morphine on C57BL/6J and Becn1 ${ }^{+/-}$glial cytokine secretion over a range of morphine concentrations (100 nM, $250 \mathrm{nM}, 500 \mathrm{nM}$, and $1000 \mathrm{nM}$ ) for 24 hours of exposure (Figure 2.8). While no significant difference to MCP-1 (Figure 2.8b) secretion was observed across the various concentrations of morphine, there were concentration dependent fluctuations in RANTES secretion from C57BL/6J glia and IL-6 secretion from both C57BL/6J and Becn1+/- glia (Figure 2.8a \& 2.8c). In addition, Becn1+/- glia showed reduced levels of chemokine secretion as compared to C57BL/6J glia, while strain differences in IL-6 secretion were not significant. Despite these changes, overall, morphine-induced cytokine secretion is quite minimal and not comparable to that of Tat induced secretion making morphine treatment alone experimentally irrelevant (Figure 2.8).

It has been shown that glial co-exposure to Tat and morphine provokes the enhanced secretion of cytokines and chemokines which contribute to the neuroinflammation characteristics of neuroAIDS [242, 261]. Moving forward with $500 \mathrm{nM}$ morphine which has been previously shown to saturate MORs [261], we exposed C57BL/6J and Becn1 ${ }^{+/-}$glia to Tat alone and in combination with morphine to confirm whether Beclin 1 is involved in the interactive effects of Tat and morphine-induced cytokine/chemokine secretion. MCP1, RANTES, and IL-6 secretion were induced by Tat treatment to C57BL/6J and significantly less in Becn1+/- glia (Figure 2.8). Co-exposure with morphine significantly 
enhanced Tat-induced cytokine secretion from both strains of glia. Interestingly, coexposure with Tat and morphine in Becn1+/- glia showed a significant reduction of about 1.8-fold in RANTES and a significant reduction of about 1.5 fold in MCP-1 secretion when compared to similarly treated C57BL/6J glia (Figure 2.8a \& 2.8b; red and blue arrows) suggesting that $60 \%$ reduction in Beclin 1 protein expression is sufficient to significantly reduce Tat and morphine-induced RANTES and MCP-1 secretion. Despite this reduction in cytokine secretion from Becn1+/- glia, these cells are still expressing approximately $40 \%$ of the Beclin 1 protein which may explain the enhancement in inflammatory molecules detected with combination Tat and morphine treatment. Co-treating the murine glia with heat inactivated Tat and morphine showed significant reduction of RANTES and MCP-1 secretion and minimal induction of IL- 6 secretion, further supporting the interactive
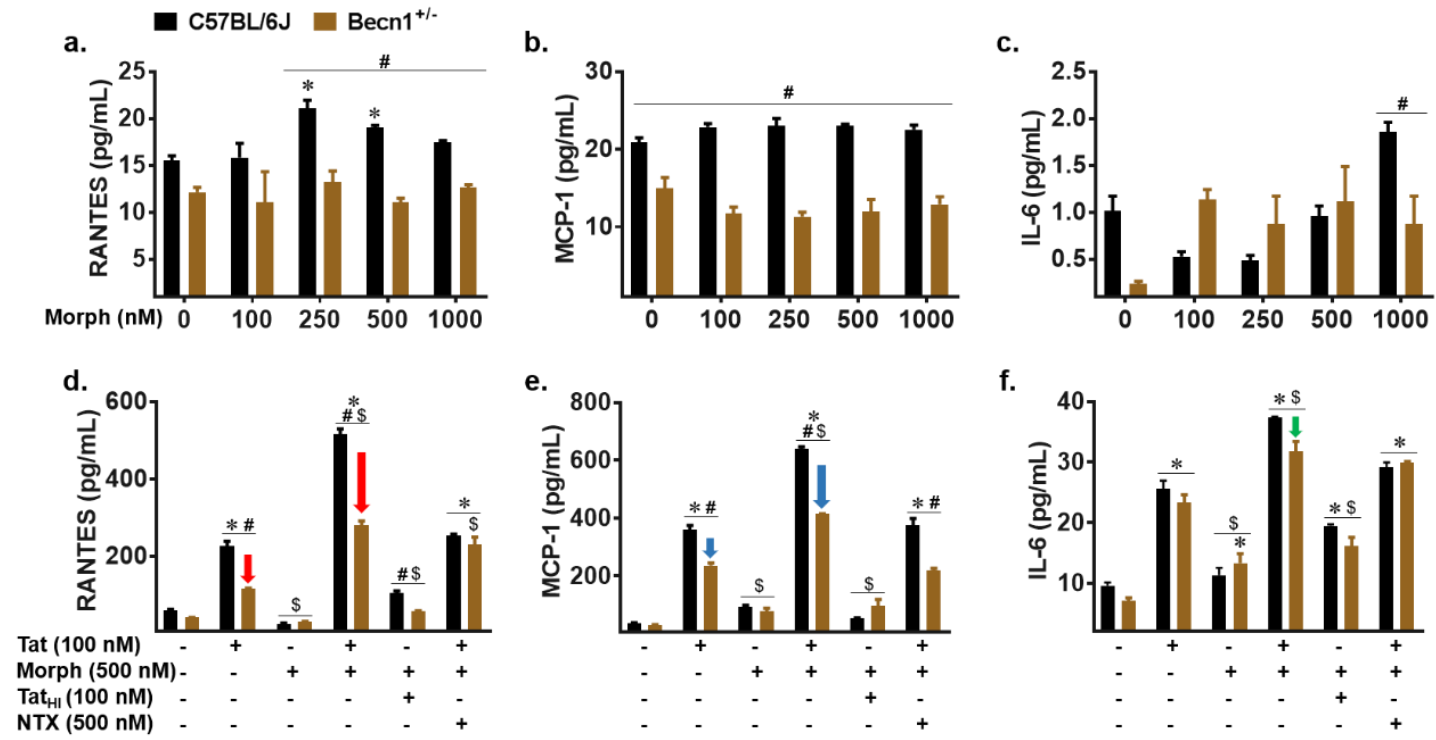

Figure 2.8 Tat and morphine induced inflammatory effects show contribution of Beclin 1 Cell culture supernatants from $\mathrm{C} 57 \mathrm{BL} / 6 \mathrm{~J}$ and Becn $1^{+/}$derived glia after indicated treatment for 24hours were assessed for cytokine and chemokine secretion by ELISA (a-f). Cytokine secretion was assessed following 24-hour treatment with increasing concentrations of morphine: $100 \mathrm{nM}, 250 \mathrm{nM}$, $500 \mathrm{nM}$, and $1000 \mathrm{nM}(\mathrm{a}-\mathrm{c})$. Tat and morphine co-exposure were assessed after 24-hours (d-f). Arrows indicate reduction in RANTES ( $d$, red), MCP-1 (e, blue), and IL-6 (f, green) secretion from Becn $1^{+/}$derived glia relative to C57BL/6J derived glia for given treatment. Values were determined from standard curves and are presented as the mean \pm SEM of 4 independent experiments. $P<$ 0.05 * vs. Control; \# vs. C57BL/6; \$ vs. Tat. Two-way ANOVA followed by Tukey's test. 
effects of morphine and active Tat. Using the MOR antagonist naltrexone prior to morphine administration was able to significantly decrease RANTES, MCP-1 and IL-6 secretion to the levels of Tat alone indicating the interaction is acting through MOR. Of note, Tat and morphine enhanced MCP-1 release in Becn $1^{+/-}$glia was significantly reduced to the level of Tat alone with pre-treatment of naltrexone, implying Beclin 1 and MOR dependent mechanisms (Figure 2.8e).

Looking further at the actions of Tat and morphine occurring at the MOR which is widely present throughout the brain, particularly the striatum, mRNA levels were measured by qRT-PCR (Figure 2.9a). Analysis showed that with Tat treatment, mRNA levels of MOR were increased by 3.5 -fold in C57BL/6J glia whereas in Becn $1^{+/}$glia, there was only a slight decrease in MOR as compared to the untreated control. The co-exposure of Tat and morphine to C57BL/6J glia also caused an increase in MOR mRNA, albeit, not as robust as Tat alone treatment. Addition of naltrexone to Tat and morphine treatment resulted in mRNA levels like that of combined Tat and morphine. Interestingly in Becn $1^{+/}$glia, Tat and morphine treatment led to higher MOR mRNA levels than that of Tat alone which was reduced to the level of Tat by naltrexone; however, this was not significantly different from untreated control. In addition, we also looked at how other opioid receptors might be contributing to these effects given that there is often co-stimulation of the different opioid receptors through off-target effects [96]. C57BL/6J glia treatment with Tat had significant effects on the mRNA levels of both the DOR (Figure 2.9b) and KOR (Figure 2.9c). When combined with morphine treatment, the trend observed was similar that that of the MOR with decreased levels compared to that of Tat alone though not significant. Intriguingly, these effects on DOR and KOR were less pronounced in the Becn $1^{+/}$treated glia with Tat treatment causing mild upregulation of DOR and showing no effects on KOR. 

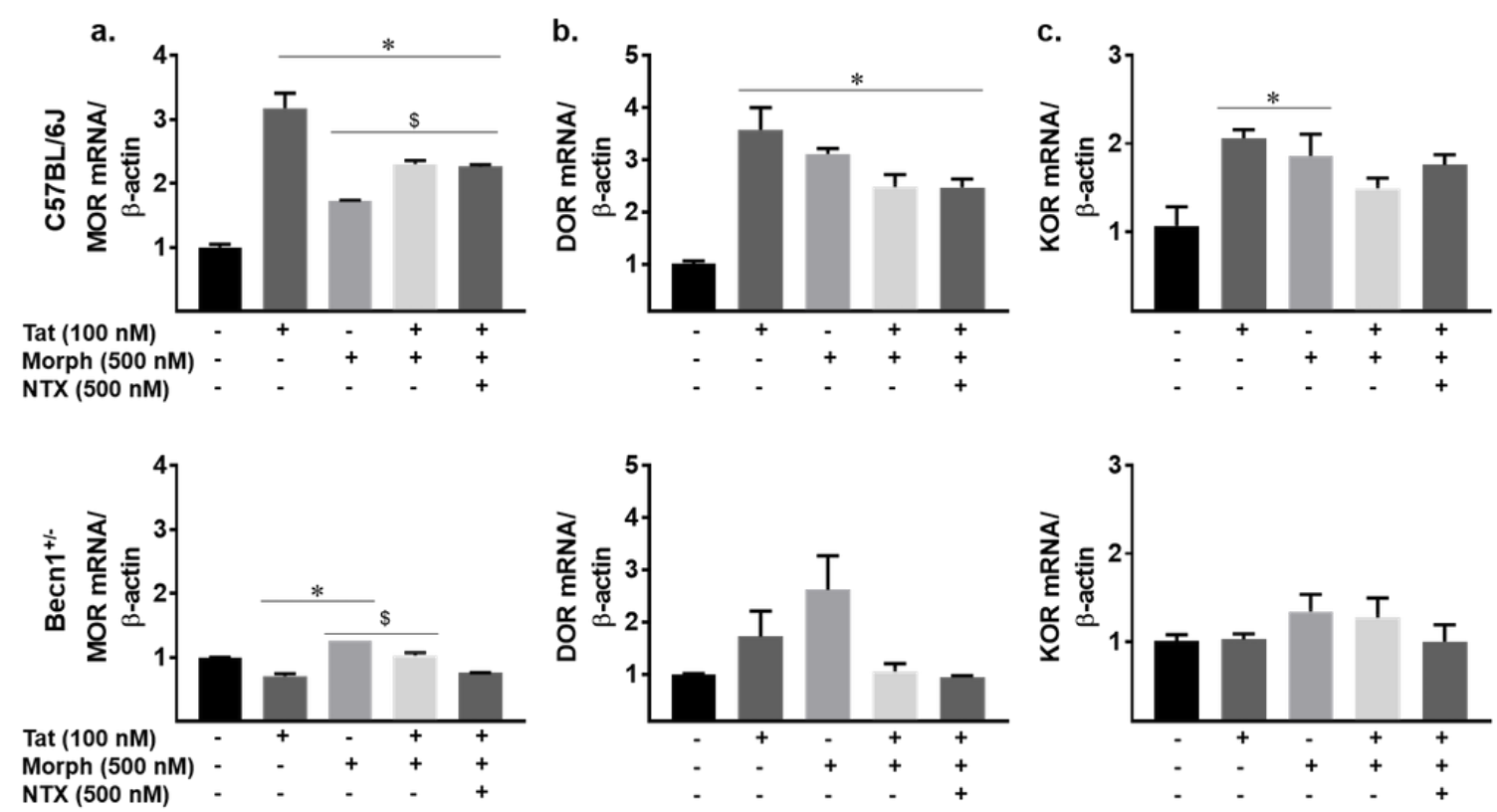

Figure 2.9 Differential effects on opioid receptor mRNA upon Tat and morphine exposure mRNA levels of murine $\mu$-opioid (MOR), $\delta$-opioid (DOR), and $\mathrm{k}$-opioid (KOR) were assessed by qRT-PCR following treatment with Tat and/or morphine in the presence and absence of naltrexone. Values were determined by $2^{\wedge}-\Delta \Delta C T$ method and normalized to $\beta$-actin. Error bars show SEM for 3 independent experiments. $\mathrm{P}<0.05$ * vs. Control; \# vs. C57BL/6; $\$$ vs. Tat. One-way ANOVA followed by Tukey's test.

To ensure the combined treatment was not drastically or differentially reducing the viability of the glia and therefore reducing cytokine/chemokine production or mRNA, an MTT assay was performed to determine cell viability across a range of morphine concentrations (Figure 2.10). Approximately $20 \%$ cell death was observed with each of the treatments, with no significant difference between concentrations of morphine. This reinforces the idea that the effects of Tat and morphine on microglia and astrocytes are on cellular function rather than inducing toxicity in regard to both the wild type and mutant cells. Overall, from the data it can be taken that the autophagy pathway intercedes in Tat and morphineinduced cytokine production. It is important to note that the observed $60 \%$ reduction of Beclin 1 protein expression (Figure 2.1) is sufficient to reduce Tat-induced inflammation but not sufficient to completely prevent the enhanced inflammation detected in Becn $1^{+/-}$ 
glia when co-exposed to morphine and Tat as compared to Tat-treated glia, suggesting that additional mechanism(s) may be at work in regulating the release of inflammatory molecules in conjunction with Beclin 1 and the autophagy pathway, or that complete or $>60 \%$ protein reduction is required for better inhibition of neuroinflammation.

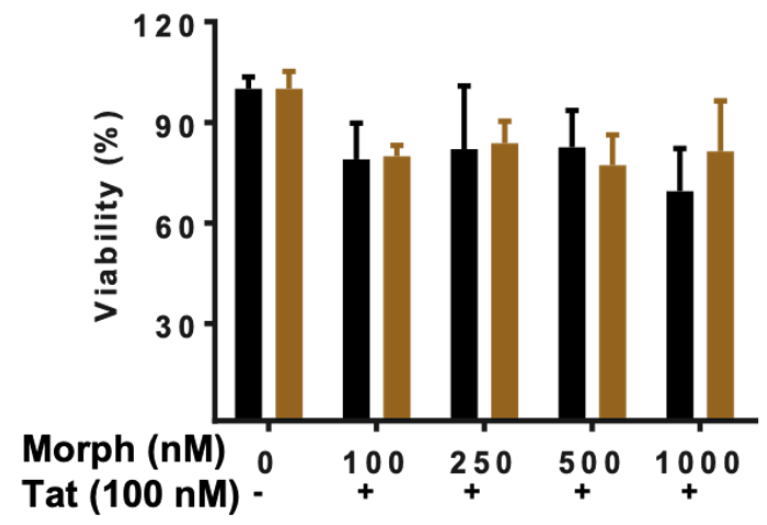

Figure 2.10 Glial toxicity of Tat and morphine

Toxicity of combined Tat and increasing morphine dose in C57BL/6J and Becn $1^{+/}$derived glia was assessed by MTT assay. Data are presented as percent viability compared to Tat treated cells (a). Error bars show SEM for 3 independent experiments. $\mathrm{P}<0.05^{*}$ vs. Control; \# vs. C57BL/6; \$ vs. Tat. Two-way ANOVA followed by Tukey's test.

\subsubsection{Tat and morphine use Beclin 1 to alter the autophagy pathway}

After determining the effects of Tat and morphine on cytokine release (Figure 2.8) and the concentration dependent effects of Tat on autophagy protein expression (Figure 2.9), we were interested to see how the autophagy pathway might be altered upon co-exposure with morphine. Beclin 1 expression was elevated with Tat treatment in both strains, 2-fold in C56BL/6J and 1.4-fold in Becn1+/- (Figure 2.11a,b). Co-exposure to morphine increased C57BL/6J Beclin 1 expression as compared to control; however, there was no enhancing interactive effect as compared to Tat. Morphine was seen to cause a slight elevation in Beclin 1 expression in both C57BL/6J and Becn1 ${ }^{+-}$derived glia. Interestingly, Tat and morphine co-exposure to Becn1+/- glia caused no change in Beclin 1 expression compared 
to control. The expression ratio of LC3 II/LC3 I showed small fluctuations following Tat and morphine treatments alone or in combination from which conclusions on autophagosome formation may not be drawn (Figure $2.11 \mathrm{c}, \mathrm{d}$ ). Surprisingly, morphine treatment to Becn $1^{+/ /}$glia reduced the LC3 II/ LC3 I ratio which may be suggestive of either reduced lipidation and reduced autophagosome formation or enhanced autophagic flux. Upon analysis of p62/SQSTM1, we observed Tat caused increases in expression within C57BL/6J glia that were not seen in Becn1+/- glia (Figure 2.11e,f) taken as accumulation of p62/SQSTM1 and halt of autophagic degradation. Similar to what was seen with Beclin 1 expression, no additive effects on p62/SQSTM1 expression were seen with Tat and morphine co-exposure for either C57BL/6J or Becn $1^{+/}$glia; however, where C57BL/6J was slightly elevated with co-exposure, the Becn $1^{+/}$was on level with control. Although not considered an autophagy protein, we also looked at expression of lysosomalassociated membrane protein1 or LAMP1, a transmembrane protein predominantly located across lysosomal membranes which acts as a glycoprotein carbohydrate ligand [268]. We found that C57BL/6J treatment with Tat alone or in combination with morphine increased the expression of LAMP1 which correlated with the observed changes in p62/SQSTM1 expression (Figure 2.12g,h). Coupling C57BL/6J LC3 and p62/SQSTM1 expression, the data suggests that where Tat alone can prevent p62/SQSTM1 and thus autophagosome degradation, when in combination with morphine, the increase in LC3 II/I and p62/SQSTM1 may indicate increased number of autophagosomes which are also not being degraded. This is also supported by the increased expression of LAMP1 with Tat and morphine treatment which may imply an increased number of lysosomes or autolysosomes. By comparison, the reduced LC3 II/I and unchanged p62/SQSTM1 expression in Becn $1^{+/}$glia treated with Tat alone or in combination with morphine, imply a degree of ongoing autophagic flux despite the reduction of Beclin 1. Analysis of 
autophagy-related gene expression at the RNA level by qRT-PCR yielded no significant differences from Tat and/or morphine exposure within each strain (data not shown). The data provide evidence that Tat alone or in combination with morphine can initiate the autophagy pathway but prevent autophagosome degradation by the lysosome, which in the context of HIV, would allow for enhanced viral replication. However, by reducing the levels of Beclin 1, autophagosome degradation may persist, albeit at lowered levels.

a.
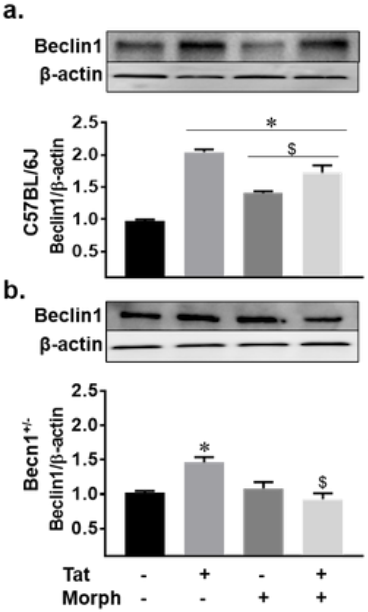

c.
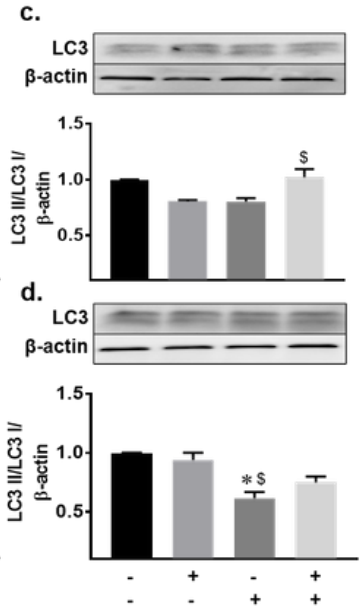

e.
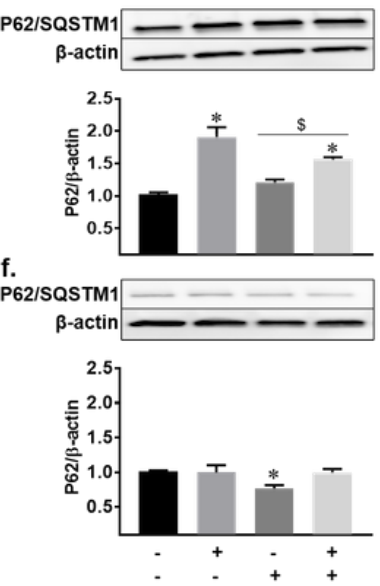

g.
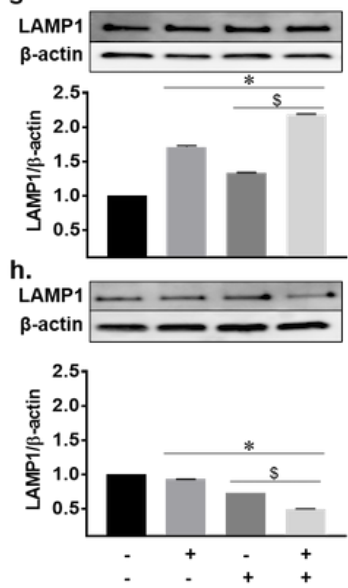

Figure 2.11 Beclin 1-mediated autophagy dysregulation by Tat and morphine

Whole cell lysates from C57BL/6J and Becn $1^{+/}$derived glia following 24 hours of the indicated treatments were subjected to immunoblotting with antibodies to Beclin 1 (a-b), LC3 (c-d), p62/SQSTM1 (e-f), and LAMP1 (g-h). Densitometry was performed for quantification on separate blots, and the ratios of each protein to $\beta$-actin are presented graphically. Error bars show the SEM of 3 independent experiments. $\mathrm{P}<0.05^{*}$ vs. Control; \# vs. C57BL/6J; \$ vs. Tat. One-way ANOVA followed by Tukey's test.

\subsubsection{Tat and morphine induced effects on intracellular calcium are mediated by}

\section{Beclin 1}

Although astrocytes are non-excitable compared to neurons, activation by ligands such as glutamate can trigger $\mathrm{Ca}^{2+}$ oscillations in glial cells leading to $\mathrm{Ca}^{2+}$ waves. These waves can be used as a means of communication amongst astrocytes and with surrounding neurons [269]. Astrocytic waves can not only modulate neuronal cytosolic $\mathrm{Ca}^{2+}$ but may also trigger the release of gliotransmitters like glutamate, ATP, D-serine, TNF-alpha, and 
arachidonic acid metabolites [270]. Calcium excitotoxicity is a feature of HIV-induced neuronal death pathology which can be stimulated by inflammatory and oxyradical stressors potentially causing altered glutamate and NMDAR signaling and excessive release of calcium stores from the endoplasmic reticulum [271-274]. Previous studies, as well as our own recently published data, have shown that increases in intracellular calcium caused by HIV in human astrocytes can lead to neuronal injury [246, 251]. In these studies, we use Tat, which we have previously shown to induce calcium release in brain cells [261, 264]. To assess the role of autophagy in glial calcium homeostasis we use the fluorescent indicator Fura-2. As expected, we observed a significant increase in $\left[\mathrm{Ca}^{2+}\right]$ in $\mathrm{C} 57 \mathrm{BL} / 6 \mathrm{~J}$ glia treated with Tat, which was significantly increased in the presence of morphine (Figure 2.12a). In contrast, Tat-induced $\left[\mathrm{Ca}^{2+}\right]_{i}$ was significantly reduced in Becn1 ${ }^{+-}$glia and further exacerbation with morphine was abrogated when compared to similarly treated C57BL/6J glia (Figure 2.12b). To ensure that the increased Fura-2 ratios corresponded to intracellular calcium release, we pre-treated murine glia with the intracellular calcium chelator, BAPTA/AM. Pre-treatment with BAPTA abrogated calcium levels in C57BL/6J and Becn $1^{+/}$- derived glia confirming that the increased levels in Fura-2 ratios were due to the release of intracellular calcium. This data suggests that the reduction of Beclin 1 expression allows primary murine glia to prevent Tat and morphine-induced excessive $\left[\mathrm{Ca}^{2+}\right]$ i release. 


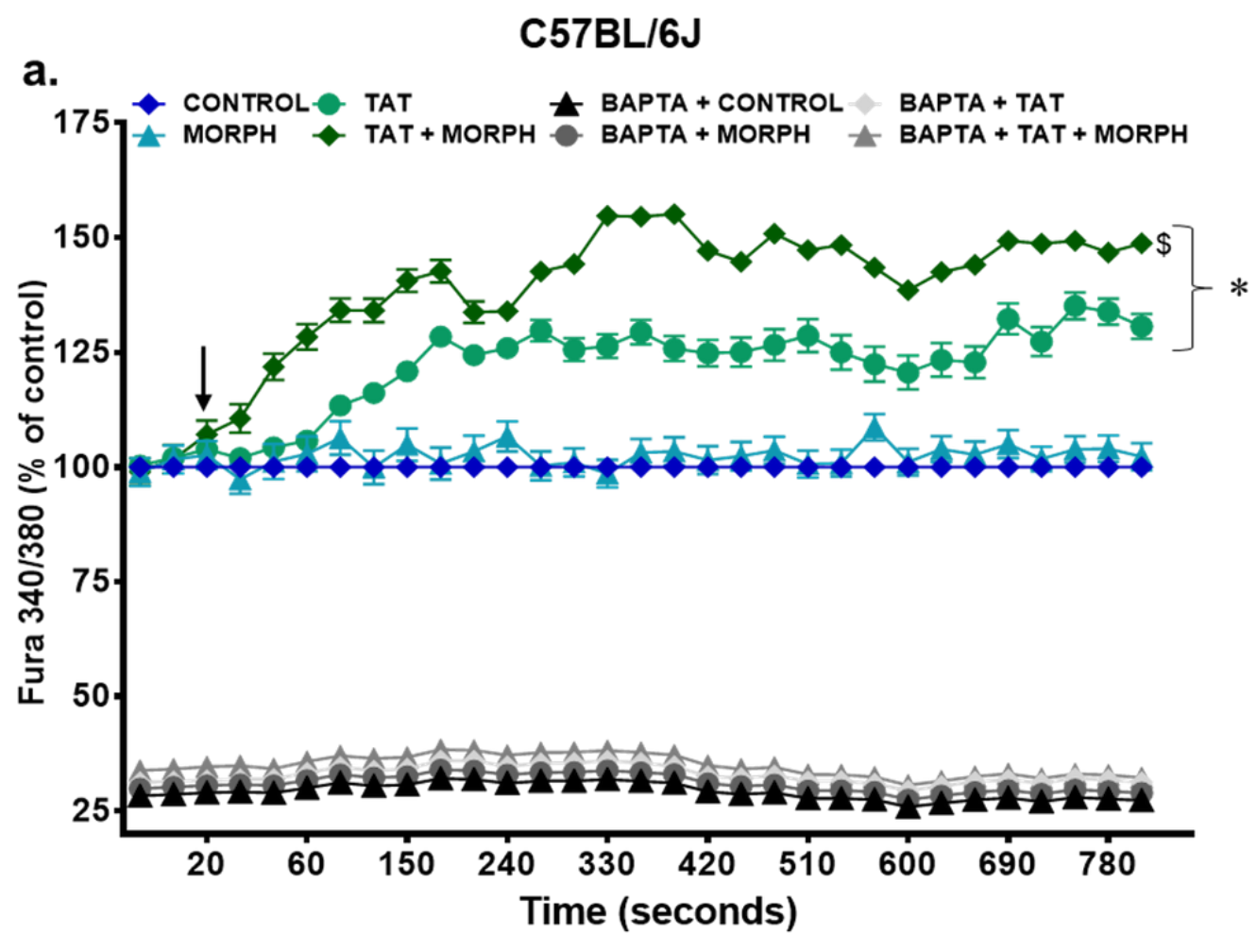

b.

\section{Becn1+/-}

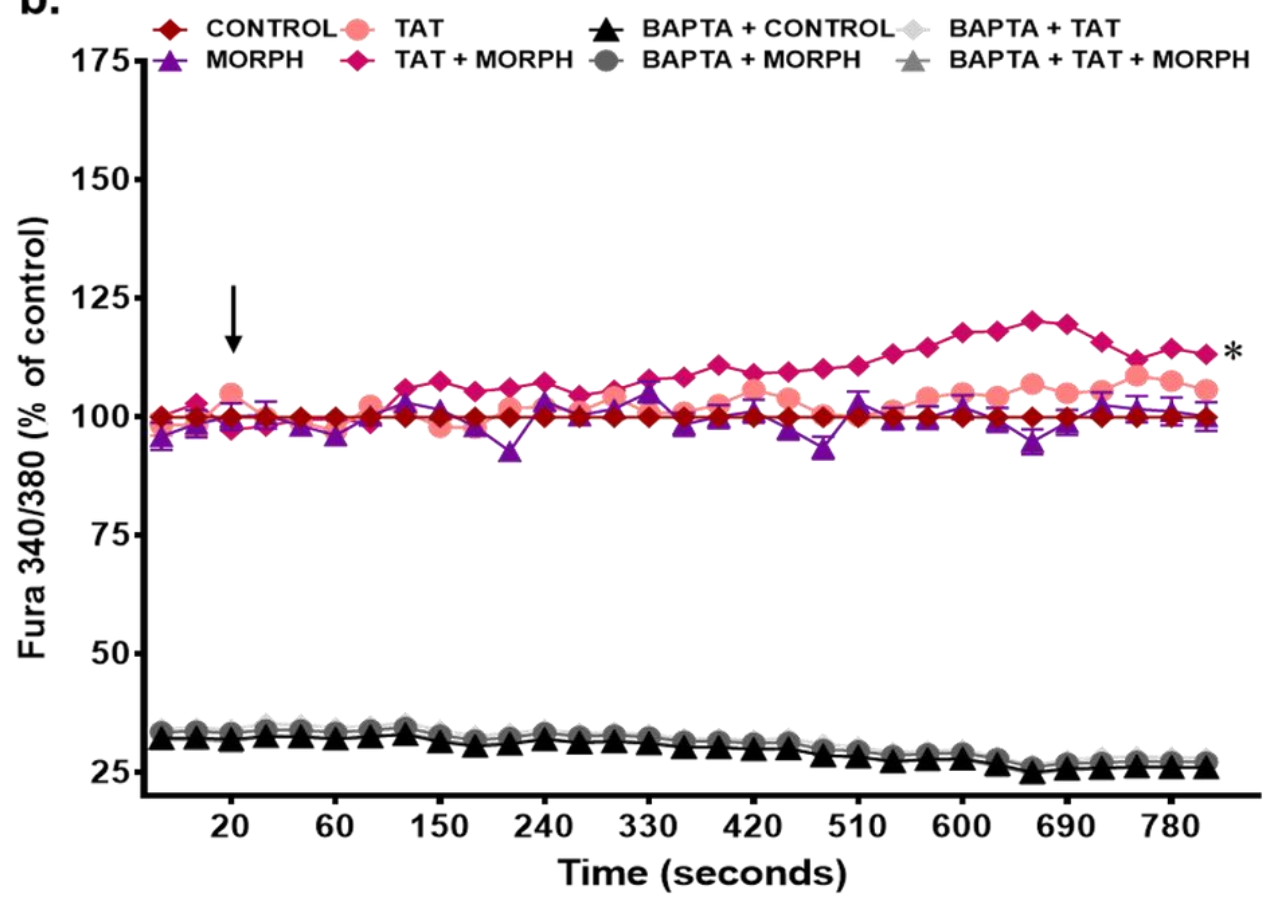


Figure 2.12 Tat and morphine enhanced intracellular calcium release is prevented by reduction of Beclin 1 expression

Intracellular calcium release from C57BL/6J (a) and Becn1+- (b) glia was assessed by Fura-2 over 800 seconds following treatment with Tat \pm morphine. The calcium channel blocker BAPTA was used as a control. Arrows indicate time of treatment. Results represent the percentage of control values and are the mean \pm SEM from 3 independent experiments. $\mathrm{P}<0.05{ }^{*}$ vs. Control; \# vs. C57BL/6J; \$ vs. Tat. One-way ANOVA followed by Tukey's test

\subsubsection{Limited Tat and morphine-interactive effects on oxidative stress are not mediated by Beclin 1}

In conjunction with neuroinflammation, glial oxidative stress is an additional pathological effect common to HAND and is often characteristic of Tat-induced glial dysfunction [275, 276]. The production of ROS and RNS, has not only been associated with the activation of intracellular inflammatory signaling, but also has been shown to cause lipid peroxidation, DNA damage, and facilitate excitotoxicity through the release of glutamate, ultimately leading to neuronal damage or death [250, 271, 277-280]. To evaluate the role of autophagy in mediating the effects of Tat and morphine on oxidative stress in mixed glia, intracellular ROS formation was assessed by DCF reactivity and reactive nitrogen species (RNS) determined nitrite accumulation. Peak levels of DCF fluorescence were recorded after 16 hours of treatment for both C57BL/6J and Becn $1^{+/}$glia (Figure 2.13). At 16 hours, Tat treatment was seen to illicit the highest level DCF fluorescence for both C57BL/6J and Becn1 ${ }^{+-}$glia with no apparent additive effect with morphine. Notably, though the increases in ROS caused by Tat were not substantial, within the strains, Tat evoked a $35 \%$ increase in ROS in C57BL/6J glia compared to untreated control (Figure 2.13a) whereas there was only a $20 \%$ increase in Becn $1^{+/}$glia (Figure $\left.2.13 b\right)$. In addition, although no enhancing effect with Tat and morphine was observed in either strain, when compared to untreated control, Tat and morphine raised ROS by $20 \%$ in C57BL/6 J derived glia compared to a $13 \%$ increase in Becn $1^{1 /-}$ derived glia. RNS assessed by NO production 
was measured by Griess assay, which is based upon the accumulation of nitrite as the product of NO metabolism. C57BL/6J glia showed no significant differences in NO accumulation upon treatment with Tat alone or in combination with morphine (Figure 2.13c) compared to untreated control. In Becn $1^{+/-}$glia, we observed a temporary spike in NO accumulation early after Tat treatment which stabilizes at later time points (Figure 2.13d). In addition, Tat and morphine causes a gradual accumulation of $\mathrm{NO}$ in Becn1+glia, which is only significantly different after 24 hours of treatment. These studies suggest an effect on ROS and NO production upon exposure to Tat but detect no interactive effect when combined with morphine with minimal intervention by Beclin 1. 

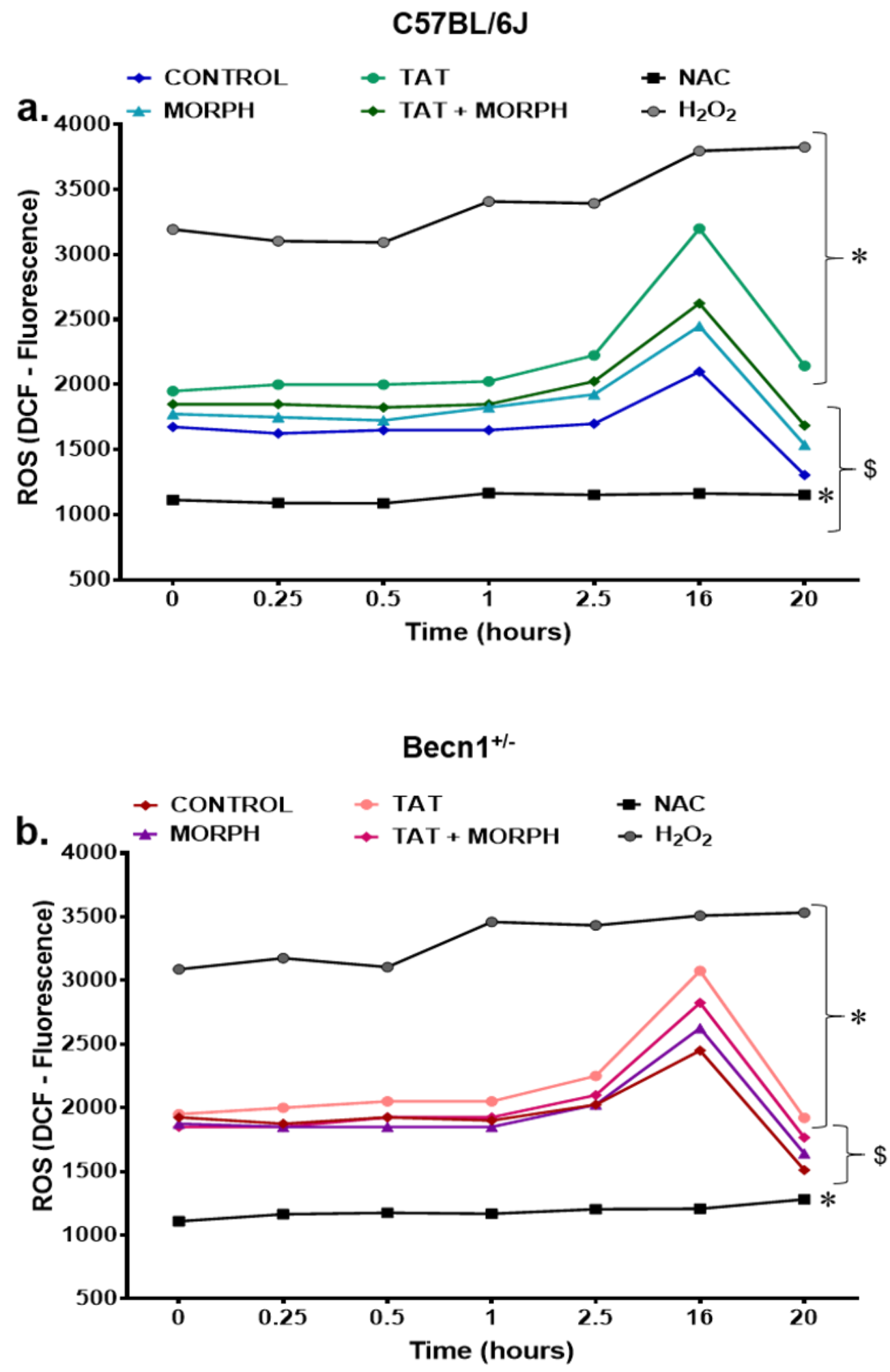

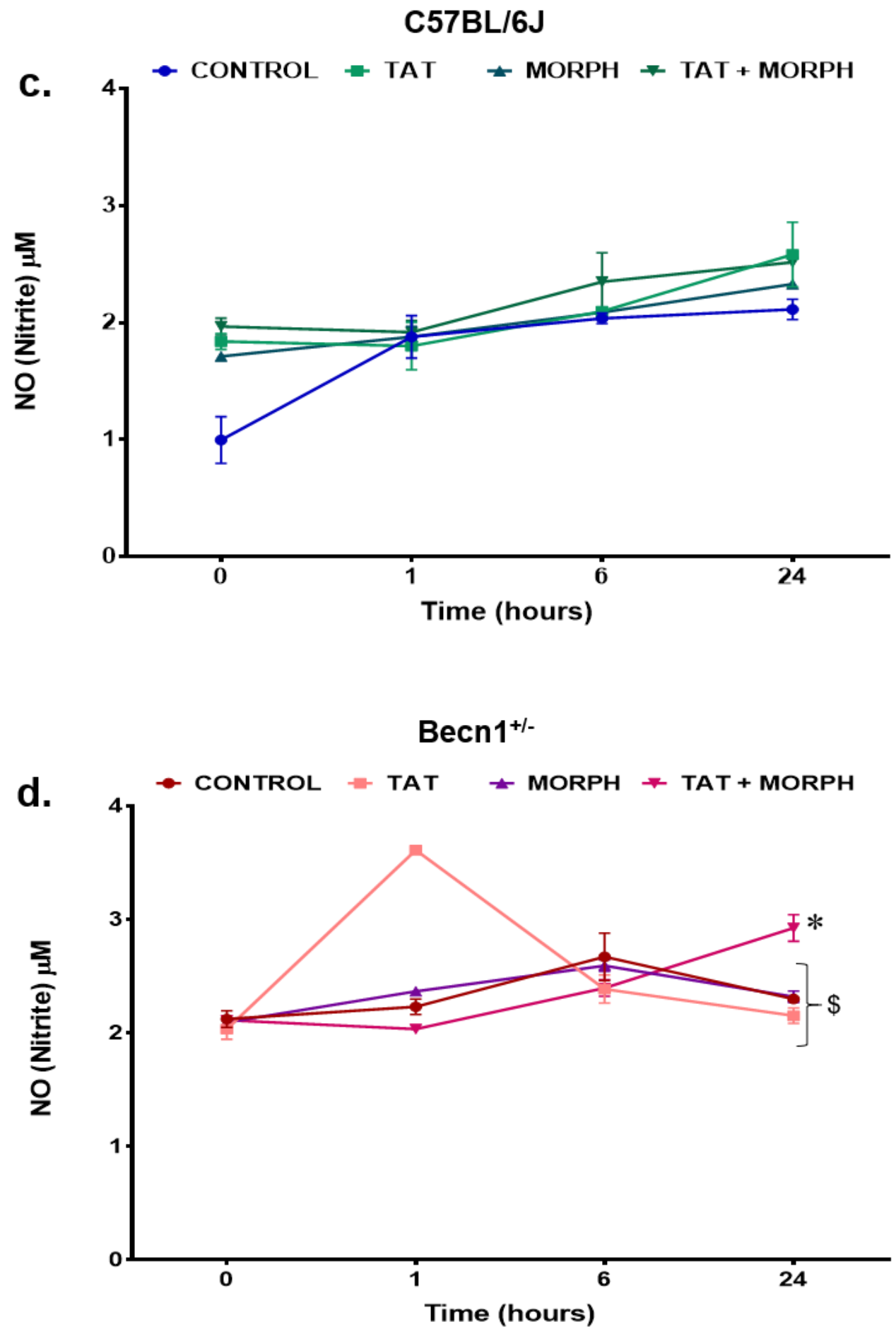
Figure 2.13 Beclin 1 is independent of oxidative stress induced by Tat with limited contribution of morphine

ROS production was assessed by dichlorofluorescein diacetate fluorescence (DCF) over a period of 24 hours following the indicated treatments to C57BL/6J (a) and Becn1+- (b) glia. Mean DCF relative fluorescence was used as an estimate of ROS and was compared for each treatment. Results are the mean \pm SEM from 6 independent experiments. NO production by C57BL/6J (c) and Becn1 ${ }^{+-}$(d) glia were examined at 0, 1, 6 and 24 hours following the indicated treatments. Nitrite levels were assessed using the Griess reaction with conversion of known concentrations of nitrate to nitrite as standards. Results represent the mean \pm SEM of 3 independent experiments. $P<0.05$

* vs. Control; \# vs. C57BL/6J; \$ vs. Tat. Two-way ANOVA followed by Tukey's test

\subsection{Discussion}

In this chapter, we use glial cells from mouse possessing a monoallelic deletion of the Becn1 gene to examine to consequences of autophagy deficiency in regulating HIV and morphine-induced inflammation, specifically through Tat. Given that homozygous deletion of the Becn 1 allele results in embryonic lethality, using a murine model with heterozygous deletion of the Becn1 allele provides a reduced autophagic environment while maintaining sufficient expression for growth and development. This model not only showed reduced expression of the autophagy protein Beclin 1 (Figure 2.1), but also showed less LC3 upon induction of autophagy by the mTOR inhibitor rapamycin (Figure 2.3). Studies in neuronal cells also indicated the necessity of initial stages of autophagosome formation for Tatinduced GFP-LC3 puncta accumulation demonstrated by Beclin 1 knockdown [191]. The Premo $^{\mathrm{TM}}$ LC3B-GFP Autophagy Sensor has been utilized in literature for the assessment of autophagosome formation in the presence and absence of autophagy inducers [281283]. Importantly, this assay only provides a static snapshot of LC3, specifically autophagosome formation, since GFP fluorescence is quenched within the lysosome. Given that LC3B-GFP puncta can result from both activation of the autophagy process or a defect in autophagosome maturation, further studies are necessary to distinguish the two events. Neuroinflammation is a well-documented characteristic of HIV associated dementia-like symptoms and plays a key role in HIV-induced neuropathogenesis [54]. In 
addition, studies have shown in vitro exacerbation of cytokine and chemokine secretion from astrocytes and microglia treated with HIV or Tat with co-exposure of morphine [242, $261,264,284]$. Kyei et al has previously shown that autophagy is used to augment viral biogenesis in macrophages by HIV [16] which provides a basis for studying the role of autophagy in mediating neurodegeneration both by HIV and in the context of drugs of abuse. Further, studies on viral proteins have illustrated not only their role in inducing neuroinflammation through the secretion of inflammatory cytokines, but also intervening in various stages of the autophagy pathway [16, 194, 285-290]. The viral protein Tat has long been demonstrated to induce damage to the brain in murine studies through cytokine driven inflammation and gliosis [199]. The penetrative capabilities of the recombinant protein across cell membranes is well documented. Extracellular Tat is able to be internalized through its basic domain within exon 1 and its RGD domain which is able to bind integrin receptors [37]. Given that Tat can enter the cell and possesses a nuclear localization signal allowing it to localize in the nucleus, there are a number of pathways such as NFKB which may be activated to induce these inflammatory effects [31, 291].

From the viral proteins we assessed, Tat was found to have the most robust inflammatory response from the C57BL/6J and Becn1 ${ }^{+/}$glia; however, other proteins did show effects (Figure 2.6). This study used the viral proteins Rev, Gag, gp120, and Nef, many of which possess neurotoxic capacity, to examine interactions with Beclin1 and whether they mediate neurotoxic effects [7]. The protein Rev is responsible for post-transcriptional gene expression within the HIV genome and has been shown to possess neurotoxic properties by disrupting membrane phospholipids [287]. The viral protein Gag, which is present in the HIV nucleocapsid, interacts with LC3, localizing virus to the autophagosome and facilitating viral proliferation within. However, the nonstructural protein Nef, a facilitator of 
viral replication, directly interacts with Beclin1, allowing the virus to escape the degradation phase of autophagy [16]. Nef causes toxicity to neurons and glial cells, in addition to inhibiting autophagy $[194,285,290]$. The envelope protein gp120 is responsible for viral entry into cells and is considered to determine viral tropism [289]. Neurotoxic in and of itself, gp120 has been shown to induce secretion of cytokines and chemokines such as IL-6, TNF- $\alpha$, RANTES, and IL-1 $\beta$ [288]. In addition, studies in neuroblastoma cells have reported gp120 to induce autophagy with increases in Beclin1 expression and LC3-GFP positive puncta [286]. While we saw no significant up regulation of the pro-inflammatory cytokine TNF- $\alpha$ with Tat, there was a marked increase when treated with the viral protein Nef which was heightened by reduction of Beclin 1. Reports have shown that Nef is able to induce cytokine and chemokine secretion in astrocytes and microglia through the mitogen pathway (p38 MAPK) and the transcription factor, NF-KB [292, 293]. TNF- $\alpha$, in particular, has been associated with elevated levels of Nef and increased infectivity in astrocytes [294, 295]. We also observed that the differences between strains which were seen with Tat treatment were absent with Nef treatment, apart from TNF- $\alpha$. This may be explained by Nef's ability to alter early endosomal compartments and vesicles, similar to that of the autophagosome in autophagy, in astrocytes [296, 297]. Comparing the effects of the individual viral proteins shown here to our laboratory's previous data demonstrating the effects silencing Beclin 1 in HIV-infected microglia and astrocytes on inflammatory molecule secretion, it is clear that the viral proteins weigh different contributions to HIV-induced neuroinflammation [192, 246].

In our present study, Tat was shown to have a concentration dependent effect on neuroinflammation through the secretion of cytokines and chemokines, with lack of Beclin 1 able to significantly attenuate these effects (Figure 2.4). This is consistent with previous 
studies by our lab showing that silencing of Beclin 1 in microglia and astrocytes reduces HIV replication as well as HIV and morphine-induced inflammation [192, 246, 257]. In addition, here we provide evidence that Tat, an established promoter of neuroinflammation, can also be modulated by autophagy within brain glia using Beclin 1 . We observe Tat concentration dependent changes to autophagy-associated proteins Beclin 1 and p62/SQSTM1 in C57BL/6J glia which are limited in the Becn ${ }^{+/-}$glia (Figure 2.7). Studies have shown that the effects of Tat on autophagy are largely dependent on conditions, concentration, and cell type, either activating or suppressing autophagy function in glial cells or neurons [191, 193, 298]. While activation of autophagy by Tat should direct the viral protein for degradation as shown in $C D 4^{+} \mathrm{T}$ lymphocytes [178], dysregulation in the degradation of the autophagolysosome may allow for persistence of Tat and autophagosome accumulation [248]. The study by Sagnier et al also found that it is possible for Tat to exhibit ubiquitin-independent, direct interaction with p62/SQSTM1. Though there is a possibility the changes to p62/SQSTM1 within our studies may be independent of ubiquitination and LC3, this would require further study. Notably, the Sagnier study was performed in $\mathrm{CD} 4^{+} \mathrm{T}$ cells and it is likely that these interactions of Tat may be cell-type specific.

The interactive effects of Tat and opiates on glial function have been previously reported by our lab and others [106, 264], yet the mechanistic role of Beclin 1-mediated autophagy has not been fully confirmed. Examining the interactive effects of Tat and morphine on cytokine and chemokine production, we observed that morphine was able to exacerbate the levels of RANTES, MCP-1, and IL-6 secretion induced by Tat, which were mediated by MOR (Figure 2.8). Although diminished Beclin 1 expression was able to significantly reduce Tat and morphine-induced cytokine secretion as compared to C57BL/6J derived 
glia, this reduction alone was not sufficient to abrogate RANTES and MCP-1 release. This agrees with our previous studies which showed significant but not complete abrogation of cytokine and chemokine release in HIV and morphine-exposed microglia and astrocytes transfected with siRNA against Beclin $1[192,246]$. Of note, previous studies by TurchanCholewo et al on the effects of Tat and morphine on opioid receptor surface expression and mRNA levels imply cell-specificity with differing effects between primary murine astrocytes and microglia and cell lines [299]. That group suggested the cell type specificity of Tat and morphine action at the MOR may determine the role of opioid receptors in brain inflammatory signaling responses, which is supported by our qRT-PCR results (Figure 2.9). Although morphine is a classical MOR ligand, we did detect alterations to DOR and KOR mRNA levels with both Tat and morphine treatments respectively. The same group found a similar trend in primary microglia with Tat-induced up regulation of DOR which was slightly decreased when combined with morphine, and little to no effect on KOR. Interestingly, in primary astrocytes Tat showed insignificant effects to DOR or KOR. Given that our system uses a mixed glial culture (astrocytes + microglia), our results may be an intermediate of those findings. Our studies also show increased opioid receptor mRNA with pre-treatment of naltrexone. This antagonist can reversibly bind MOR, and to a lesser extent DOR and KOR and has been reported to increase the density of opioid receptors, despite, by definition, being neutral or negative in efficacy [300]. It is important to note that the Turchan-Cholewo study used primary astrocytes and microglia from the forebrain unlike the striatum used in these studies. In addition, differential induction of chemokines and their receptors by Tat and morphine has been suggested to have cell type-specific bidirectional, cross-sensitization with opioid receptors which may also modulate their expression [301]. Therefore, it is possible that the Beclin 1-dependent responses to Tat and morphine are cell-type specific with the relative presence of microglia and astrocytes 
potentially masking the ameliorating effects of autophagy reduction in our mixed-glial model. Another factor to examine is cell surface receptor dynamics. In general, morphine is considered a lower efficacy agonist and may only induce receptor internalization under certain conditions in certain cell types [302]. Given that Beclin 1 has functions outside of autophagy such as endocytosis (through its interaction with UVRAG and Vsp34), it is also possible alterations in trafficking machinery causing impaired internalization of the opioid receptors may explain the Tat and morphine-induced differences in mRNA between the strains $[262,303,304]$.

The autophagy pathway can be modulated by HIV or Tat exposure, with other HIVproteins also able to modulate autophagy. Other studies have reported that upon HIV treatment, human astrocytes show increased protein expression of Beclin 1 indicative of autophagy initiation in conjunction with elevated LC3 [188]. In addition, it has been reported that Tat is able to stimulate autophagy by increasing the levels of BAG3 in human glial cells shown by increased LC3 II protein via western blot and immunofluorescence [193]. Our studies agree as we detected elevated expression of Beclin 1 in C57BL/6J derived glia treated with Tat; however, though also increased relative to untreated control, no interactive effect was detected when co-administered with morphine (Figure 2.11a). This is consistent with our previous studies in HIV-infected human astrocytes and microglia co-exposed to morphine $[192,246]$. It is of note that Tat exposure to Becn $1^{+/-}$ derived glia moderately increased Beclin 1 protein expression, albeit not as significantly, when compared with C57BL/6J derived glia and even less so with Tat and morphine treated Becn1 ${ }^{+/}$derived glia (Figure 2.11b). This seems to contradict the LC3-GFP studies in mutant glia which suggested that these cells inefficiently induce autophagy activation. A study in rat hepatocytes demonstrated that early inhibition of autophagy by the PI3K 
inhibitor 3-methyladenine prevented LC3 lipidation while maintaining bulk cargo autophagic flux [305]. It is therefore a possibility that with the $60 \%$ reduction in Beclin 1 protein expression in the mutant glial cells, may be able to carry out autophagy, if only to a lesser extent. In our studies here, we did not detect significant changes in LC3 II// ratio with Tat treatment alone or in combination with morphine (Figure 2.7b, 2.11c,d). Tat treatment has shown to increase LC3 II expression in both astrocytes and neurons [191, 193]. It is possible that these differences may be due to lack of antibody sensitivity for LC3 II which may cause the signal ratio of LC3 I and LC3 II to be skewed and improperly reflect the ratio of cytosolic and membrane-bound LC3 $[306,307]$. In addition, inhibitors of autophagic degradation such as bafilomycin $\mathrm{A} 1$ or chloroquine should also be utilized to establish LC3 II increase and thus confirm autophagosome accumulation. Despite being the most commonly utilized tool for assessing autophagy, it is important to note that LC3 expression levels alone are not sufficient to determine autophagic flux due to the fact that LC3 II is in equilibrium in terms of its formation and degradation [308]. As such, data must be analyzed in conjunction with other markers such as the adaptor protein p62/SQSTM1 or in the presence of autophagosomes [307]. Expression of LC3 and p62/SQSTM1 in Tat treated C57BL/6J derived glia was suggestive of reduced LC3 leading to the accumulation of p62/SQSTM1. When combined with morphine, p62/SQSTM1 protein levels were elevated compared to untreated control, which may indicate accumulation of autophagosomes due to impaired clearance (Figure 2.11c \& 2.11e). Notably, these effects were significantly minimized in glia derived from the Becn $1^{+/-}$mouse (Figure $2.11 \mathrm{~d} \&$ 2.11f). If autophagic activation is associated with increased numbers of autophagosomes, we would expect a corresponding increase in the number of lysosomes [268]. Here we see increased expression of the lysosome protein LAMP1 when C57BL/6J but not Becn $1^{+/-}$ glia are treated with Tat alone or combined with morphine (Figure 2.11g,h). Studies in 
human astrocytes demonstrated that late stage autophagy could be interrupted by the HIV protein Nef as characterized by LC3 and p62/SQSTM1 accumulation [194]. Similarly, although in neurons, Hui et al has reported that Tat can also disrupt endolysosome degradation [248]. Also, in HIVE patient post-mortem tissues, Zhou et al described increased expression autophagy proteins as well as the lysosomal marker LAMP1 [185]. As such, it is possible that Tat induces Beclin 1 expression to initiate autophagy in glia while concurrently preventing its own degradation through targeting of late stage autophagy (Figure 2.14). This type of hijacking of the autophagy pathway allows for the protection of viruses within the autophagosome and allows for the enhanced viral replication observed in HIV infected brain cells [309]. Our findings show that by reduction of Beclin 1-mediated autophagy, Tat and Tat and morphine-induced alterations to autophagy protein expression can be prevented and ultimately limit their effects; however, additional studies on autophagic flux are still needed to confirm impaired maturation. Subsequently, we can then determine whether autophagosome to lysosome fusion is occurring, and second determine any effects on autophagolysosome acidification. Moreover, the presence and accumulation of autophagosomes and lysosomes should be confirmed by electron microscopy. 


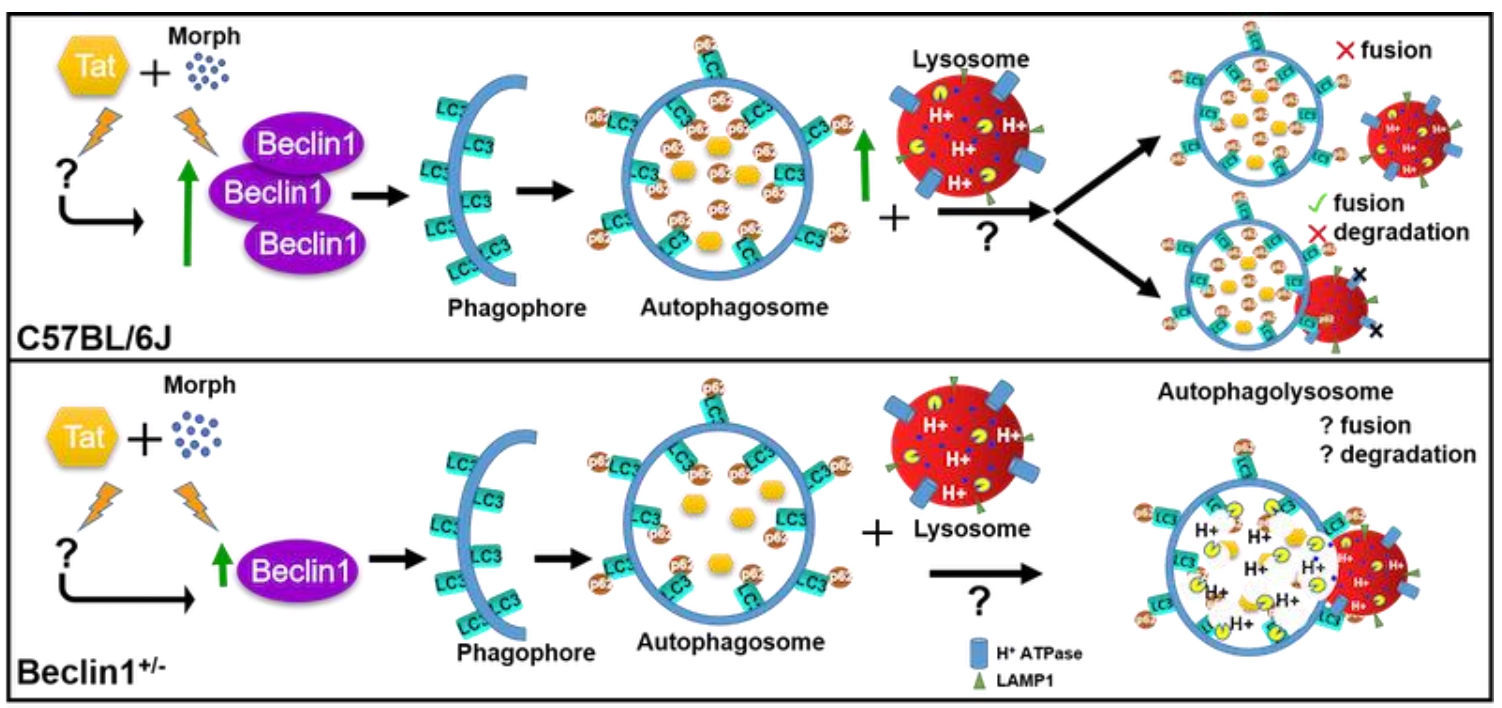

Figure 2.14 Schematic diagram summarizing the differential effects of Tat and morphine on the autophagy pathway in C57BL/6J and Beclin 1-deficient mouse glia

Green arrows indicate an induction/accumulation. Reducing the expression of Beclin 1 in mouse glia prevented excessive Tat and morphine associated induction of the autophagy pathway while also limiting the accumulation of the adaptor protein p62/SQSTM1 and the lysosomal protein LAMP1. Future studies on the fate of the autophagosome are currently pending to determine the mechanism by which the autophagy pathway is initiated by Tat and morphine, but the process halted before degradation may occur.

Tat-induced neurotoxicity is largely facilitated through the activation of the brain glia which leads to oxidative stress through excitotoxic mechanisms often involving calcium [250]. Becn $1^{+/}$glia provided further evidence supporting the role of Beclin 1 in mediating calcium release in astrocytes. Similar to what was shown in HIV-infected human astrocytes, Tat was able to induce calcium release from C57BL/6J derived glia, which was significantly enhanced by co-exposure to morphine (Figure 2.12). Moreover, Tat or Tat and morphineinduced calcium release was significantly decreased in Becn $1^{+/-}$derived glia which paralleled the siBeclin 1 treatment decreases shown in HIV-infected human astrocytes. Provided that intracellular $\mathrm{Ca}^{2+}$ is a key second messenger to numerous signaling pathways and is essential to the maintenance of glial homeostasis, it was of key importance to this study to validate the role of Beclin 1 as a potential alleviating factor for Tat and morphine-induced calcium accumulation. It should be noted that intracellular 
calcium assessments generally appear as oscillations over time with varying intensity which we did not observe in these studies. This may be due to over-loading of the Fura2-AM dye or over-exposure of the fluorescent readings, thus minimizing the oscillations for each reading point. Future studies will reduce the amount of Fura-2-AM from $5 \mu \mathrm{M}$ to $1 \mu \mathrm{M}$. We also recently showed using gene silencing against Beclin 1 and the autophagy inducer, rapamycin, a role of autophagy in buffering the release of calcium, ROS, and NO in HIV-infected human astrocytes exposed to morphine [246]. Analysis of ROS production in the present study did not detect an enhancing effect upon Tat and morphine treatment of either C57BL/6J or Becn $1^{+/-}$derived glia (Figure 2.13a,b) contradictory to our previous study with HIV-infected astrocytes or studies in microglia [107]. This may be due to saturation of the fluorescent reading which may explain the $\mathrm{H}_{2} \mathrm{O}_{2}$ kinetics. Future studies will require further optimization of the DCF dye to identify a suitable range for measurement as well as microscopy to confirm loading. Interestingly, we were able detect a slight, albeit significant, interactive effect of Tat and morphine on RNS production in Becn $1^{+/}$derived glia (Figure 2.13c-d) similarly to what was seen previously seen in HIVinfected human astrocytes [246]. It has been previously shown that interruption of the autophagy pathway may also aggravate toxicities due to the virus [310]. Using glia derived from a Beclin 1-deficient mouse we further confirmed that Tat and morphine-induced calcium, but not ROS and NO release, are mediated via a Beclin 1-mediated pathway. In conclusion, using glial cells derived from Beclin 1-deficient animal, we observe an association between the autophagy protein Beclin 1 and the HIV protein Tat, which may underlie a mechanism for glial neuropathology in the context of opiate abuse and provide insight into targeting of future therapeutics. 
CHAPTER 3: BECLIN 1 IS A REGULATOR OF NEURONAL OUTCOME AND KEY COMPONENT IN MEDIATING NEURO-GLIAL INTERACTIONS UPON TAT AND MORPHINE EXPOSURE

\section{$\underline{3.1 \text { Introduction }}$}

HIV exposure in the brain is associated with a host of neurological complications, despite advancements in cART. As research into the etiology of these deficits has expanded, the cause has been attributed to neuronal damage, dysfunction, and loss. Despite these findings, there is no evidence that supports HIV infection of neurons [311, 312]. Rather, numerous studies find that HIV exerts its harmful effects to neurons through either 1.) direct effects of viral protein products such as Tat or 2.) indirect effects mediated by the changes to the cellular environment regulated by local glial cells [66, 202, 240, 241, 290, 296, 313, 314]. In particular, the viral protein Tat is considered one of the most important arbitrators of HIV-induced neurotoxicity with studies showing that Tat exposure into the mouse striatum leads to neuronal apoptosis, synaptic destruction, dendritic pruning and gliosis [239]. Secreted into the extracellular space by infected cells and unaffected by cART, Tat is able to bind extracellular receptors or cross membranes to enter neuronal cells, ultimately wreaking havoc on neuronal signaling, membrane permeability, and initiating apoptotic cascades $[30,260]$. In addition, as discussed in the previous chapter, Tat exposure to glial populations results in glial activation, stimulation of neuroinflammation, oxidative stress, and homeostatic disruption, all of which culminate in severe stress for the neuronal bystander $[60,66,67,100,202,208,275]$. Tat triggers toxic glutamate release from glial cells which in turn activates AMPA receptors (AMPARs) and NMDARs which can trigger excitotoxicity through the opening of calcium channels and depletion of intracellular stores [315]. Moreover, in vitro studies have demonstrated that 
Tat is able to increase neuronal $\mathrm{Ca}^{2+}$ flux through interaction with NMDARs which can cause excessive neuronal signaling and damage to synapses [251, 260, 273]. Synergistic effects of Tat and morphine in neurons have also been reported to enhance neuronal death and intracellular calcium accumulation illustrating their neurodegenerative effects [101, 204, 316, 317]. Furthermore, studies using inducible Tat transgenic mice have shown a variety of pathologies depending on brain location such as astrocytosis, neuronal collapse, degeneration of dendrites, and progressive loss of the cortex [205, 207]. Considering that both the direct and indirect attacks of HIV on neurons are bolstered by opiate co-exposure, the HIV-infected/drug abusing population is at considerable risk for neuronal damage and the clinical manifestations of HAND [86, 100].

Currently, there is still much to be understood regarding the precise mechanisms of neuronal injury and death following Tat and opiate co-exposure. While effects such as excitotoxicity, mitochondrial dysfunction, dendritic damage, and neuronal apoptosis have been identified as being triggered by Tat and morphine, questions surrounding how these phenomena are being regulated remain unclear. Consequently, one such pathway which may be considered is the autophagy pathway. Studies have shown that it is possible for HIV or its proteins to induce neurodegeneration through the clearance pathway $[185,186$, 189-191]. In post-mortem brain tissues of HIV-infected persons with encephalitis, there was a marked increase in expression of autophagic proteins and autophagosome number as compared to uninfected brains or HIV brains without encephalitis [185]. Similar results were also reported when examining autophagy in the post-mortem brain tissues of HIVinfected persons with varying degrees of $\mathrm{NCl}$ prior to death as well as history of drug abuse [187]. As reviewed earlier, autophagy has associations to both HIV pathogenesis and numerous neurodegenerative diseases, with dysregulation of the pathway frequently 
leading to significant pathology. This is particularly important for post-mitotic cells such as neurons which rely on the pathway for excessive/damaged protein and organelle removal [183]. In addition, the balance of autophagy lies deeply intertwined with initiation of apoptosis and may provide the driving force in determining neuronal outcome. It is on this basis that we sought to determine how the autophagy protein Beclin 1 mediates the neuronal responses triggered by Tat and morphine co-exposure.

\section{$\underline{3.2 \text { Materials and Methods }}$}

\subsubsection{Timed-pregnancy and primary murine neuron cell cultures}

Approximate timed pregnancies were utilized to retrieve embryonic neurons at stages of high neurogenesis. Briefly, male and female mice aged 8 to 15 weeks were kept isolated for at least 24 hours prior to mating. After 24 hours, females were introduced into the breeding cage with the males in the early evening. Female mice were monitored periodically for 48 hours after initial introduction to detect for the presence of a vaginal plug indicating intercourse. The day on which the plug is observed is considered day zero and mouse weight is recorded every 3 days to ensure pregnancy.

Primary murine striatal neurons were obtained from the embryos of anaesthetized pregnant dams at a gestational age of approximately E15-E17. Tail clips of individual murine embryos were taken for genotyping confirmation of strain as described in chapter 2.2.2. Brains from each embryo was considered as an independent sample $(n=1)$. Growth medium contained Neurobasal medium ${ }^{\mathrm{TM}}$ (Gibco/Life Technologies, Grand Island, NY) supplemented with B-27 ( $2 \% \mathrm{v} / \mathrm{v}$; Gibco/Life Technologies), L-glutamine $(0.5 \mathrm{mM})$, and $1 \%$ penicillin/streptomycin. The striatal area, which contains mostly medium spiny neurons, was dissected from whole brain and cell isolation performed similarly to as 
described in chapter 2.2.3 for primary murine glia. Cells were plated and maintained in 24well plates or 4-well chambers coated with poly-L-Lysine for 5 to 7 days.

\subsubsection{Reagents and treatments}

Morphine sulfate (Sigma-Aldrich, St. Louis, MO) and Tat ${ }_{1-86}$ IIIB (ImmunoDiagnostics, Woburn, MA) were used to treat $\mathrm{C} 57 \mathrm{BL} / 6 \mathrm{~J}$ and $\mathrm{Becn} 1^{+/}$neurons at the concentrations described in chapter 2.2.7. Supernatant from C57BL/6J and Becn1+/- primary glia treated with Tat and morphine alone or in combination for 24 hours as described in chapter 2.2.7 was collected and added to neuronal cultures to assess neuronal survival. Conditioned media (CM) from treated primary glia was centrifuged and filtered through a $0.22 \mu \mathrm{m}$ filter to remove cellular debris leaving only soluble secretions and diluted 4-fold in neuronal growth media. Conditioned media (CM) was used to treat cultured C57BL/6J-derived neurons and untreated C57BL/6J neurons served as controls.

\subsubsection{Time-lapse assessment of neuronal survival}

Time-lapse digital images were assessed as described previously [257]. Briefly, images of neurons were recorded using an inverted microscope with an automated computercontrolled stage and environmental chamber $\left(37^{\circ} \mathrm{C}, 5 \% \mathrm{CO}_{2}\right)$ (Zeiss). For each well, 6-10 non-overlapping fields were selected. A total of $15-30$ individual medium spiny striatal neurons were identified in each field by their distinctive morphology in digital images. Time-lapse images of the same series of fields were recorded at 30-minute intervals for 36 hours after concurrent Tat and/or opiate treatment. At the end of each experiment, all preselected neurons were assessed for viability in digital images taken at each time point during the entire treatment period. Neuronal death was identified visually by the collapse and fragmentation of the cell body. Neuronal survival is reported as the percentage of 
viable cells at a given time point compared to the initial time point 0 . Phase contrast images were taken at 20x magnification.

\subsubsection{Cell viability}

Viability of neurons was confirmed using a live/dead cell fluorescence assay, which combines fluorescent reagents to yield two-color discrimination of the population of live cells indicated by green fluorescence, from the dead-cell population indicated by red fluorescence (ScienCell Research Laboratories). Cells were imaged using an inverted fluorescence microscope. Neuronal viability was also assessed by trypan blue exclusion assay and measured using an automated cell counter (Bio-Rad).

\subsubsection{Growth factor array}

Glial growth factor secretion was measured using the RayBio® C-Series Mouse Growth Factor Array 3 (catalog \#: AAM-GF-3-8; RayBiotech). C57BL/6J and Becn1+- glial cells were treated with Tat and morphine alone and in combination under reduced serum conditions (1\% FBS) for 24 hours. Collected supernatant was used to semi-quantitatively detect the levels of 30 mouse proteins present in the cell culture media according to manufacturer's instructions. Signal levels were detected by chemiluminescence and intensities normalized to positive control on each membrane and membranes normalized to the untreated media reference array. Results are presented as fold change relative to untreated media. 


\subsubsection{Intracellular calcium $\left[\mathrm{Ca}^{2+}\right]_{\mathrm{i}}$ measurements}

Levels of $\left[\mathrm{Ca}^{2+}\right]$ i release in neuronal cultures were measured as described in chapter 2.2.13. Data is presented as percent of control values \pm SEM from 4 separate experiments (15-25 cells).

\subsubsection{Immunohistochemistry}

Chambers containing treated neurons were immunolabeled as described in chapter 2.2.4. The primary antibody used was anti-MAP2 (Millipore, MAB378) at a 1:100 dilution for neuron visualization. Immunoreactivity was visualized with secondary antibodies from Molecular Probes (Carlsbad, CA, USA). Cells were mounted with ProLong® Gold antifade reagent with DAPI to label cell nuclei (Thermo Fisher Scientific). Images were analyzed using a Zeiss (Germany) inverted fluorescence microscope with a 560 Axiovision camera at 40x. Microtubule-associated protein 2 (MAP-2) fluorescently labeled neurons were used to assess dendritic beading following 24 hours of Tat \pm morphine treatment. Neurons were assessed blindly from three independent experiments. The total number of beads were counted and divided by the total number of neurons assessed per treatment for each experiment.

\subsubsection{Statistical analysis}

Results are reported as mean \pm SEM of 3-6 independent experiments. Data were analyzed using analysis of variance (ANOVA) techniques, followed by post hoc testing for multiple comparisons and/or repeated measures (GraphPad Prism 7 software, La Jolla, CA). A value of $p<0.05$ was considered significant. 


\section{$\underline{3.3 \text { Results }}$}

\subsubsection{Neuronal Beclin 1 deficiency contributes to aberrant Tat-induced intracellular}

calcium accumulation and dendritic injury

Neuronal signaling is heavily reliant on ion flux which regulates much of its functionality such as action potential firing and neurotransmitter release [318]. One of the most essential and highly regulated of these ions is intracellular calcium. The capability of Tat to have excitatory $\mathrm{Ca}^{2+}$ mobilization effects on neurons through interactions with both plasma membrane receptors and intracellular stores is well documented, along with highlighting the exacerbating effects of co-exposure with opiates ultimately leading to excitotoxicity $[75,316,319]$. Given the results on $\mathrm{Ca}^{2+}$ flux we observed in the mixed glial cultures (Figure 2.12), here we sought to determine what role, if any, Beclin 1-mediated autophagy plays in neuronal Tat and morphine-induced $\mathrm{Ca}^{2+}$ dysregulation. For these and future purposes, we isolated primary striatal neurons from the embryonic pups of C57BL/6J and Becn1//- bred mice (Figure 3.1). Tail clips were taken from the E15-E17 pups for genotyping as described in Chapter 2.2.2. As expected, Tat treatment to C57BL/6J neurons caused a significant increase in $\left[\mathrm{Ca}^{2+}\right]_{\mathrm{i}}(1.63 \mathrm{x}$ increase) which was elevated when co-exposed with morphine (2.05x increase) when compared to the
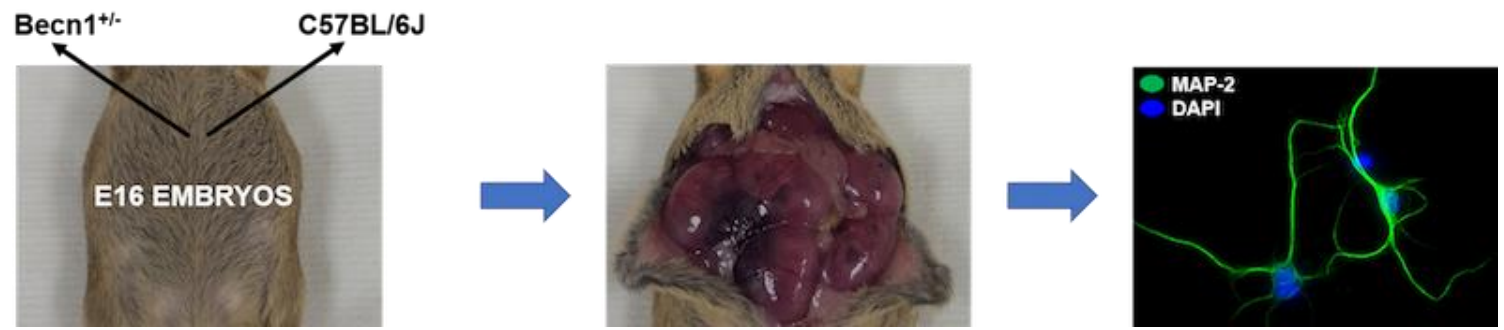

Figure 3.1 Primary culture and imaging of C57BL/6J and Becn $1^{+/-}$neurons

Primary neurons were isolated from the striatum of pups of cross-bred pregnant dams (C57BL/6J $x$ Becn $1^{+/}$) at approximately E16. Genotyping on embryonic tails was performed to confirm strain. Morphology differences between strains were assessed by immunostaining with the neuronal marker MAP-2 (green). Nuclei are stained with DAPI (blue). 
untreated C57BL/6J control (Figure 3.2a). To our surprise, Tat treatment to Becn1+/neurons also caused a significant increase in $\left[\mathrm{Ca}^{2+}\right]_{\mathrm{i}}(2.72 \mathrm{x}$ increase) compared to untreated Becn1 ${ }^{+/}$neurons (Figure 3.2b). This was significantly higher than the Tatinduced increase seen in C57BL/6J neurons. Interestingly, while the combined addition of Tat and morphine to Becn1+/- neurons also caused an increase in $\left[\mathrm{Ca}^{2+}\right]_{i}$ compared to the untreated control (2.44x increase), the effect was less than that of Tat treatment alone. Morphine alone showed no significant effects to either C57BL/6J or Becn1+/-neurons. The calcium chelator BAPTA served as a negative control. This data suggests that reduced autophagy by proxy of Beclin 1 deficiency may intercede in $\mathrm{Ca}^{2+}$ signaling leading to intracellular accumulation which may be a hindrance to neuronal survival through excitotoxicity. 


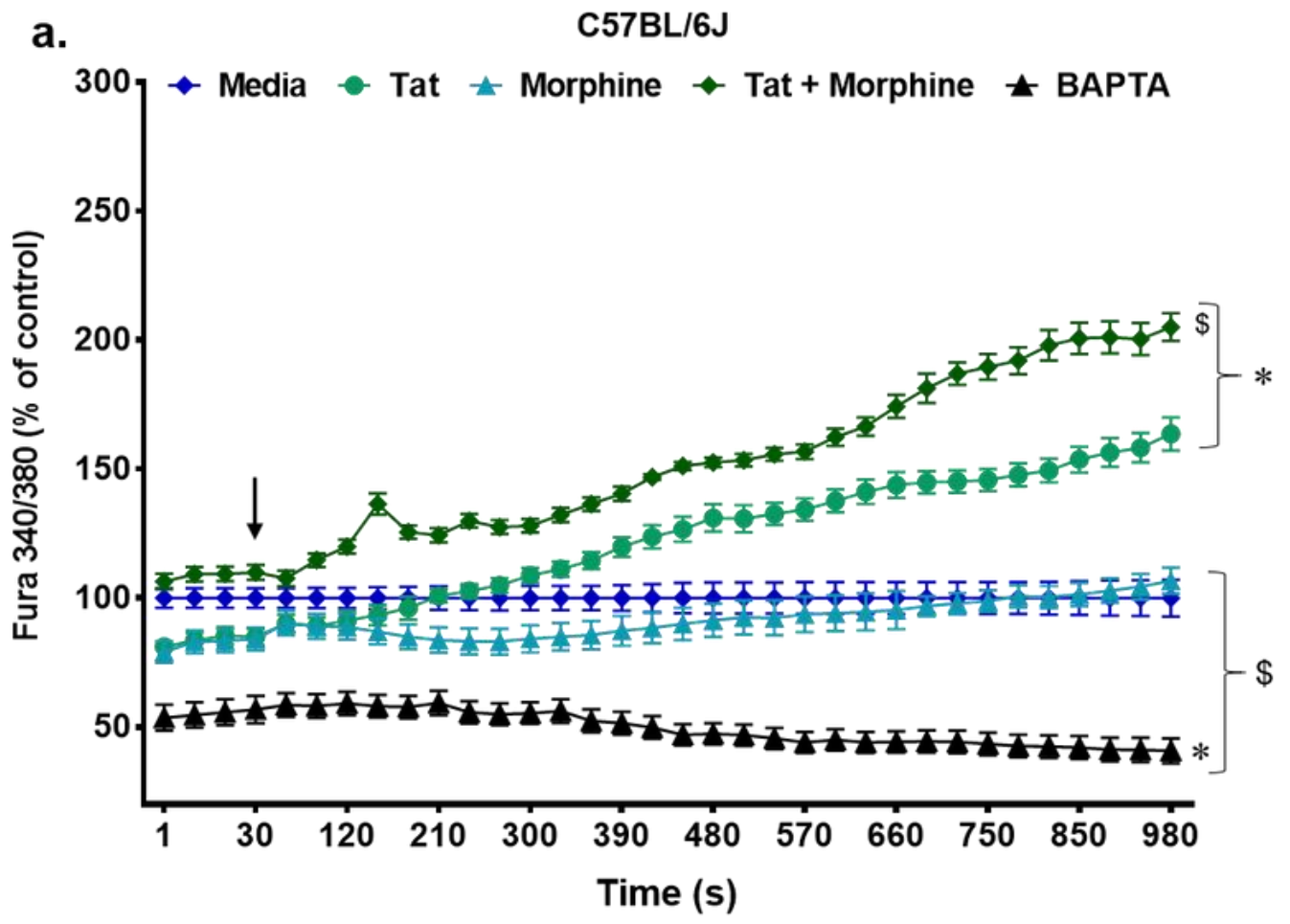

b.

Becn1 ${ }^{+/-}$

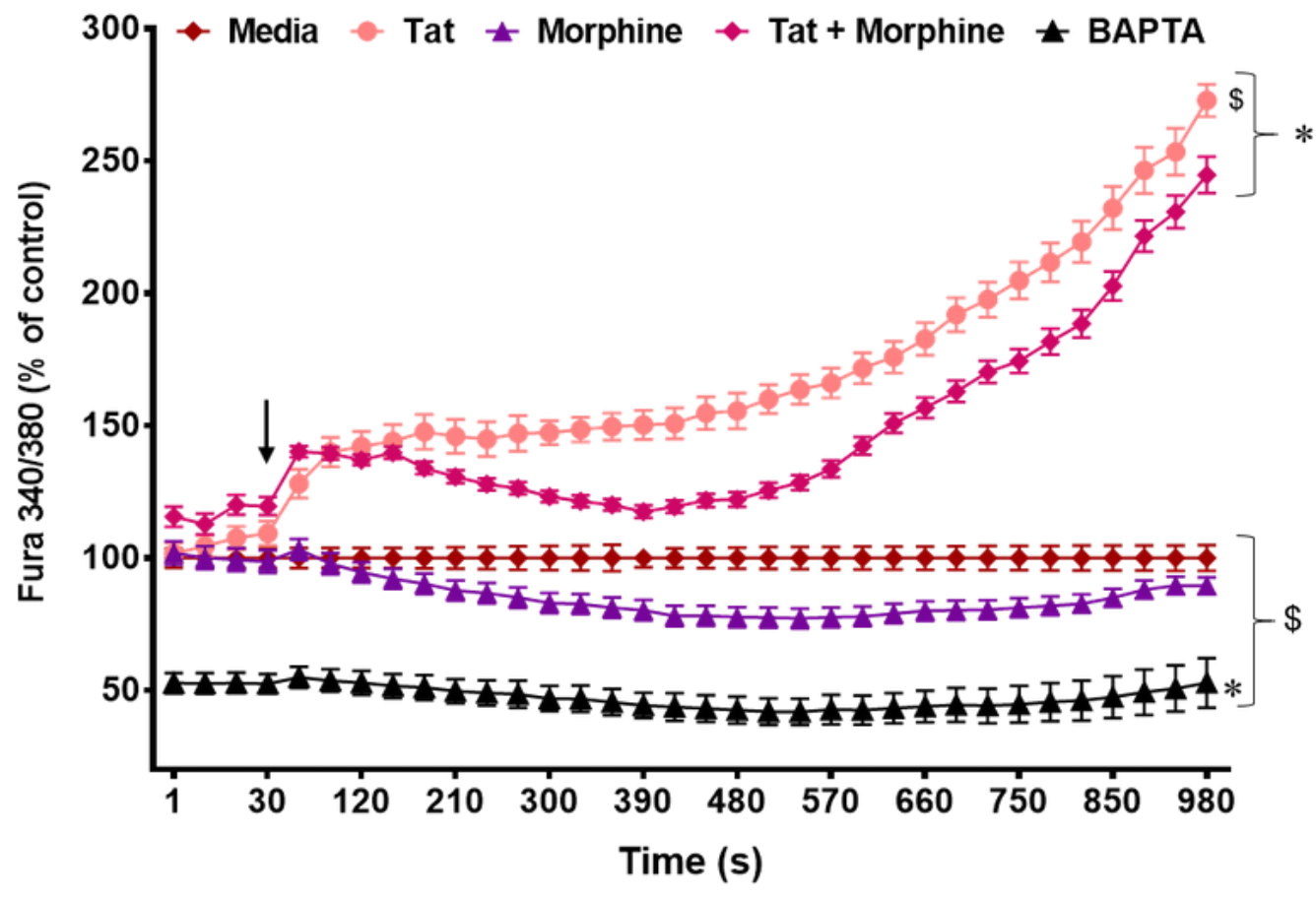


Figure 3.2 Becn1 ${ }^{+/-}$neurons exhibit increased intracellular calcium accumulation upon Tat exposure

Intracellular calcium release from C57BL/6J (a) and Becn1+/- (b) neurons was assessed by Fura-2 over 980 seconds following treatment with Tat \pm morphine. The calcium chelator BAPTA-AM was used as a control. Arrows indicate time of treatment. Results represent the percentage of control values and are the mean \pm SEM from 4 independent experiments. $\mathrm{P}<0.05{ }^{*}$ vs. Control; \# vs. C57BL/6J; \$ vs. Tat. One-way ANOVA followed by Tukey's test.

As neurons are subjected to stress within the environment, intracellular signaling cascades and responses are triggered which often manifest as structural changes. An early morphological hallmark for neurotoxicity is the presence of beading or varicosities along neurites. A response to axonal and/or dendritic injury, its presence tends to parallel neuronal death and is observed in many neurological diseases $[318,320]$ and has also been reported as a result of HIV injury [321]. As such, we continued to examine the effects of neuronal Beclin 1 deficiency upon Tat and morphine neurotoxic challenge using immunocytochemistry. MAP-2 fluorescently immunolabeled neurons were observed qualitatively for neurite beading after 24 hours of Tat and/or morphine treatment (Figure 3.3a; white arrows). Comparing the untreated C57BL/6J and Becn $1^{+/-}$groups, the mutant neurons had a significant degree of beading without any neurotoxic exposure (2.36-fold higher). Tat treatment significantly increased neurite beading in both C57BL/6J and Becn1 ${ }^{+/}$neurons (2.82 and 2.29-fold increase compared to respective strain controls). Combined Tat and morphine treatment to C57BL/6J neurons caused a similar degree of neurite beading as observed in Tat treated neurons (2.64-fold increase compared to untreated control). Tat and morphine co-exposure to Becn1+/- neurons also caused neurite beading; however, to a lesser degree than Tat treatment alone (1.32-fold increase compared to control) (Figure 3.3b). Beading caused by morphine treatment alone was slight and considered a negligible increase. Taken together, it appears as though lack of Beclin 1 drives neurons to an excitotoxic/stressed state with a heightened sensitivity to neurotoxins such as Tat. 


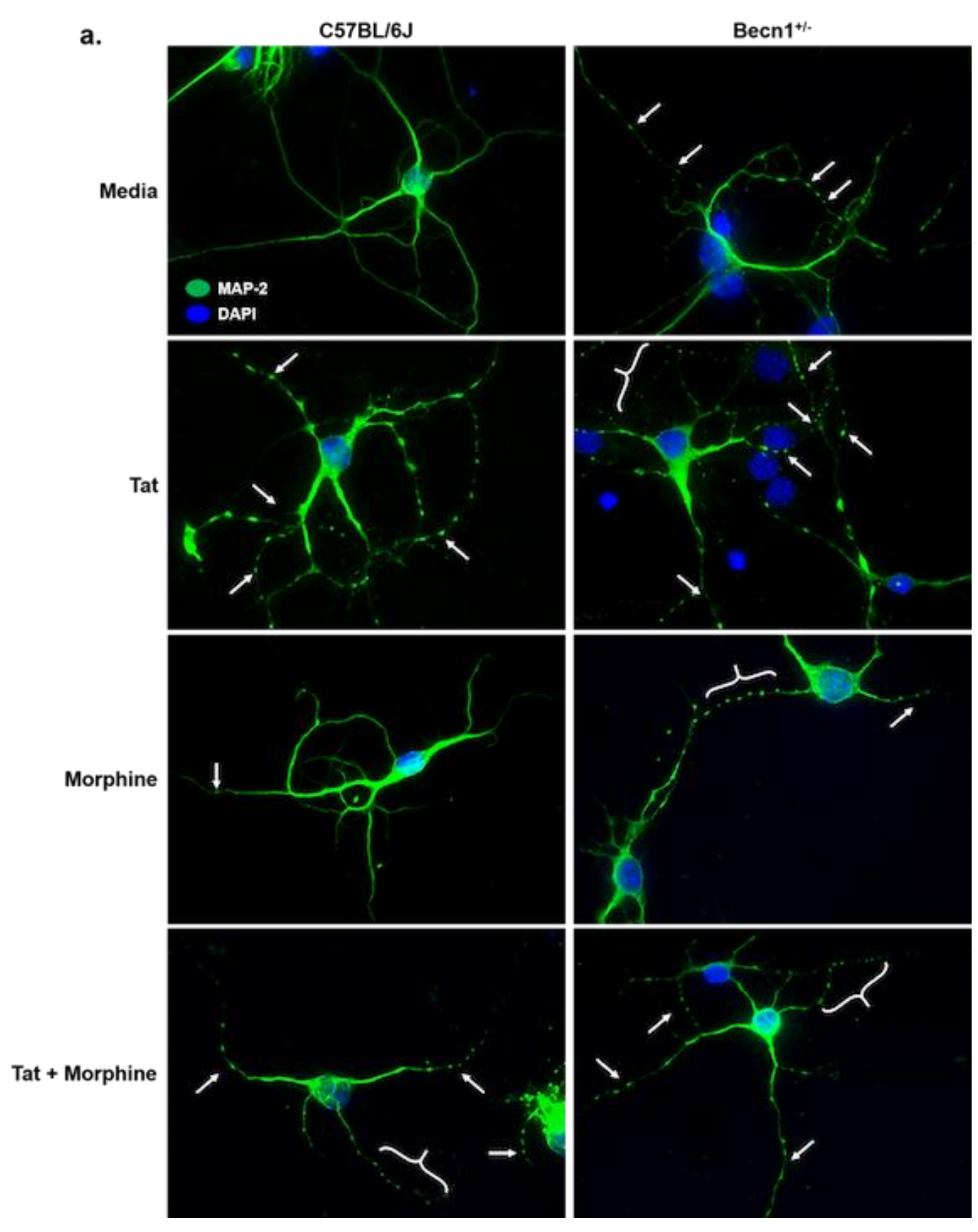




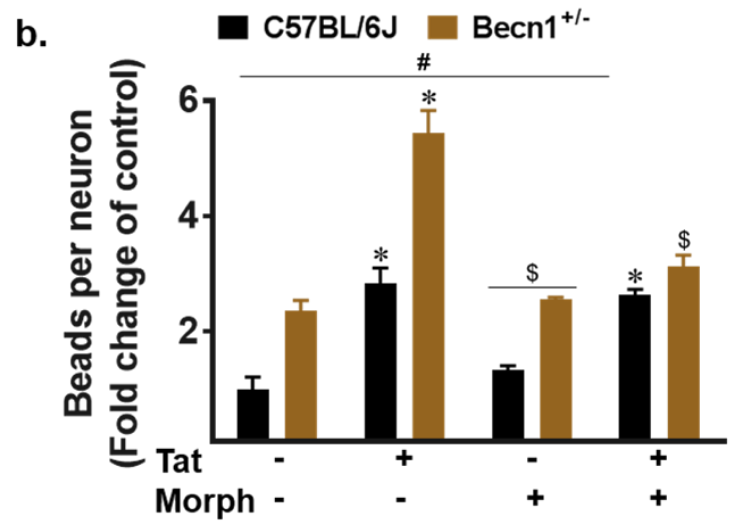

Figure 3.3 Tat exposure to Becn $1^{+/-}$neurons induces elevated dendritic beading $\mathrm{C} 57 \mathrm{BL} / 6 \mathrm{~J}$ and Becn1+/- neurons treated with Tat \pm morphine for $24 \mathrm{~h}$ were immunolabeled with neuronal marker MAP-2 to identify morphological changes and dendritic beading indicative of injury (a). Total number of beads per treatment was quantified and averaged over total number of neurons assessed per treatment per experiment. Error bars show mean \pm SEM for 3 independent experiments. $\mathrm{P}<0.05$ * vs. Control; \# vs. C57BL/6J; \$ vs. Tat. Two-way ANOVA followed by Tukey's test.

\subsubsection{Becn $1^{+/}$derived neurons show reduced viability with Tat and morphine exposure}

Neuronal autophagy is a key process in the maintenance of neurological health with aberrant or dysregulated activity being associated with the development of CNS disorders such as Alzheimer's, Parkinson's, and Huntington's diseases [322, 323]. While glial contributions to neuroinflammation have been documented to have adverse effects on neuronal health and outcome, studies have shown that Tat can also directly induce toxicity and neuronal cell death which is enhanced by morphine co-exposure $[87,100,102,104$, 204, 241, 324, 325]. In addition, previous studies on the effects of Tat and morphine on primary human neurons indicated inhibition of autophagy, reduced endolysosome $\mathrm{pH}$, accumulation of autophagosomes, and an observed obstruction of autophagosome to lysosome fusion [187, 248]. Given what we have observed in regard to the effects of reduced Beclin 1 on neuronal $\mathrm{Ca}^{2+}$ and dendritic injury, we sought to examine the role of 
Beclin 1-mediated autophagy in Tat and morphine-induced neuronal outcome. Individual neurons were tracked over 36 hours using environmentally controlled time-lapse imaging to monitor morphological changes and cell death over time (Figure 3.4a) $[86,102,326]$. Tat treatment to $\mathrm{C} 57 \mathrm{BL} / 6 \mathrm{~J}$ derived neurons showed only a slight, but statistically significant, decrease in viability (5\%) that was further decreased when combined with morphine (11\%) after 36 hours of exposure as determined by repeated measures two-way ANOVA (Figure 3.4b). No significant changes were seen with morphine treatment alone. Although mild toxic effects were induced in the C57BL/6J neurons by Tat and morphine, we observed marked increases in neuronal losses from Becn $1^{+/}$neurons from an early time point regardless of treatment when compared to C57BL/6J neurons (Figure 3.4c). Tat exposure caused a decrease in neuronal viability (10\%); however, when combined with morphine, there was no significant enhancement of neuronal death (12\%) comparable to that which was seen in C57BL/6J neurons. Neuronal viability was confirmed by fluorescent live/dead staining (Figure 3.4d) and trypan blue exclusion. Although the effects to C57BL/6J neuronal viability were not as drastic as have been reported in other studies, these results agree with existing literature in regard to the toxicity of Tat and morphine on neurons. In addition, these time-lapse results are analogous to what was observed in the neuronal calcium and dendritic beading studies with Tat and morphine co-treated Becn $1^{+/-}$neurons. This supports the concept that a $60 \%$ reduction in Beclin 1-mediated autophagy has detrimental effects to neuronal health and enhances 
injury when faced with stress, thus further confirming the essential role of Beclin 1 and autophagy in neuronal protection, which can significantly be hindered by Tat exposure.

a.
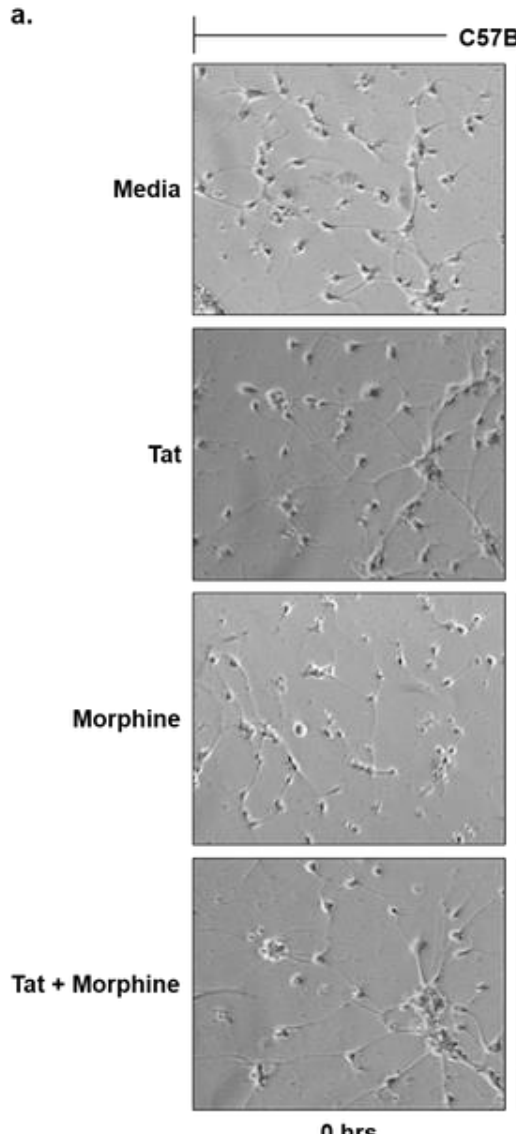

$0 \mathrm{hrs}$
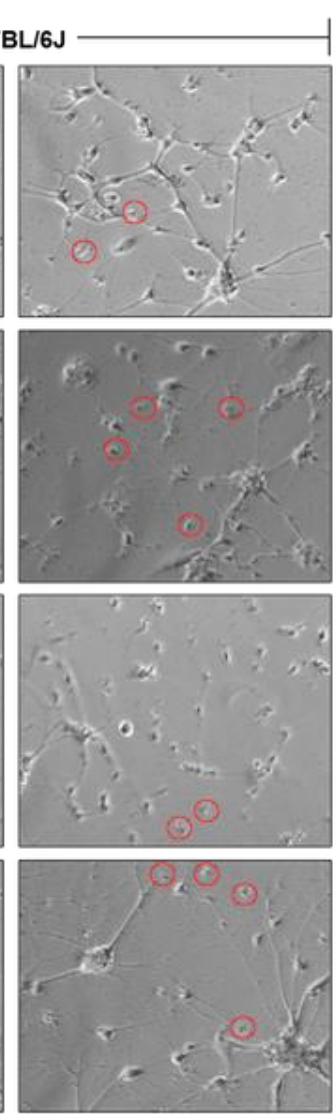

36 hrs
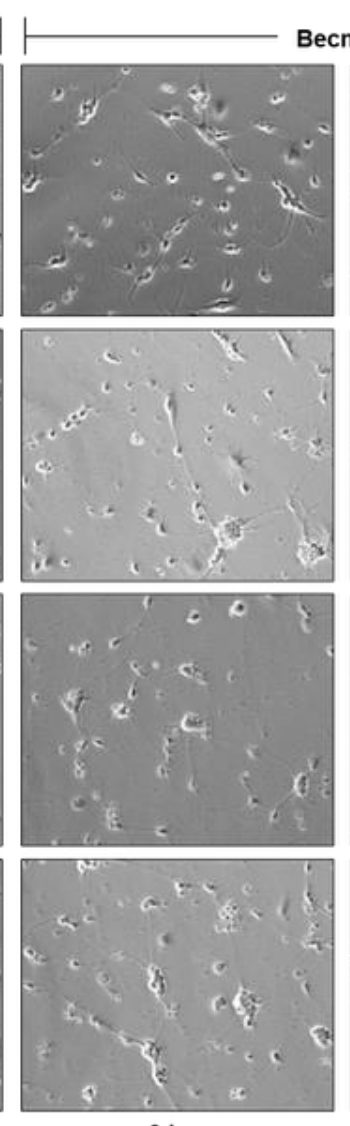

$0 \mathrm{hrs}$
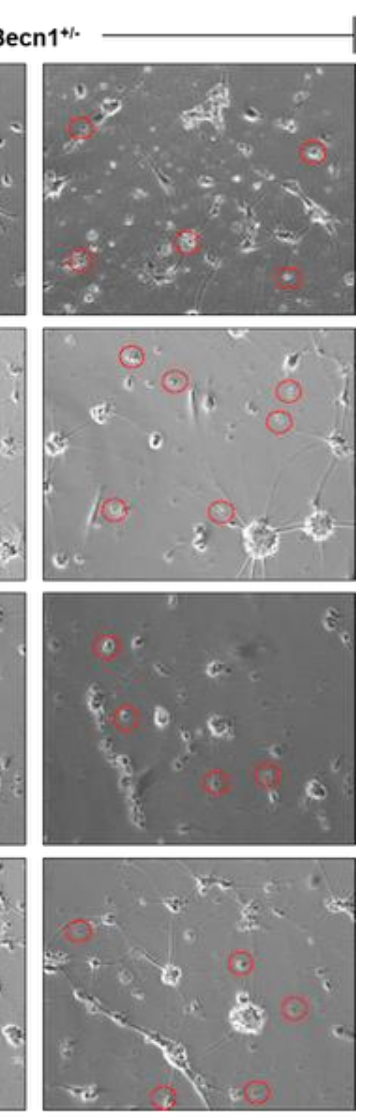

$36 \mathrm{hrs}$ 

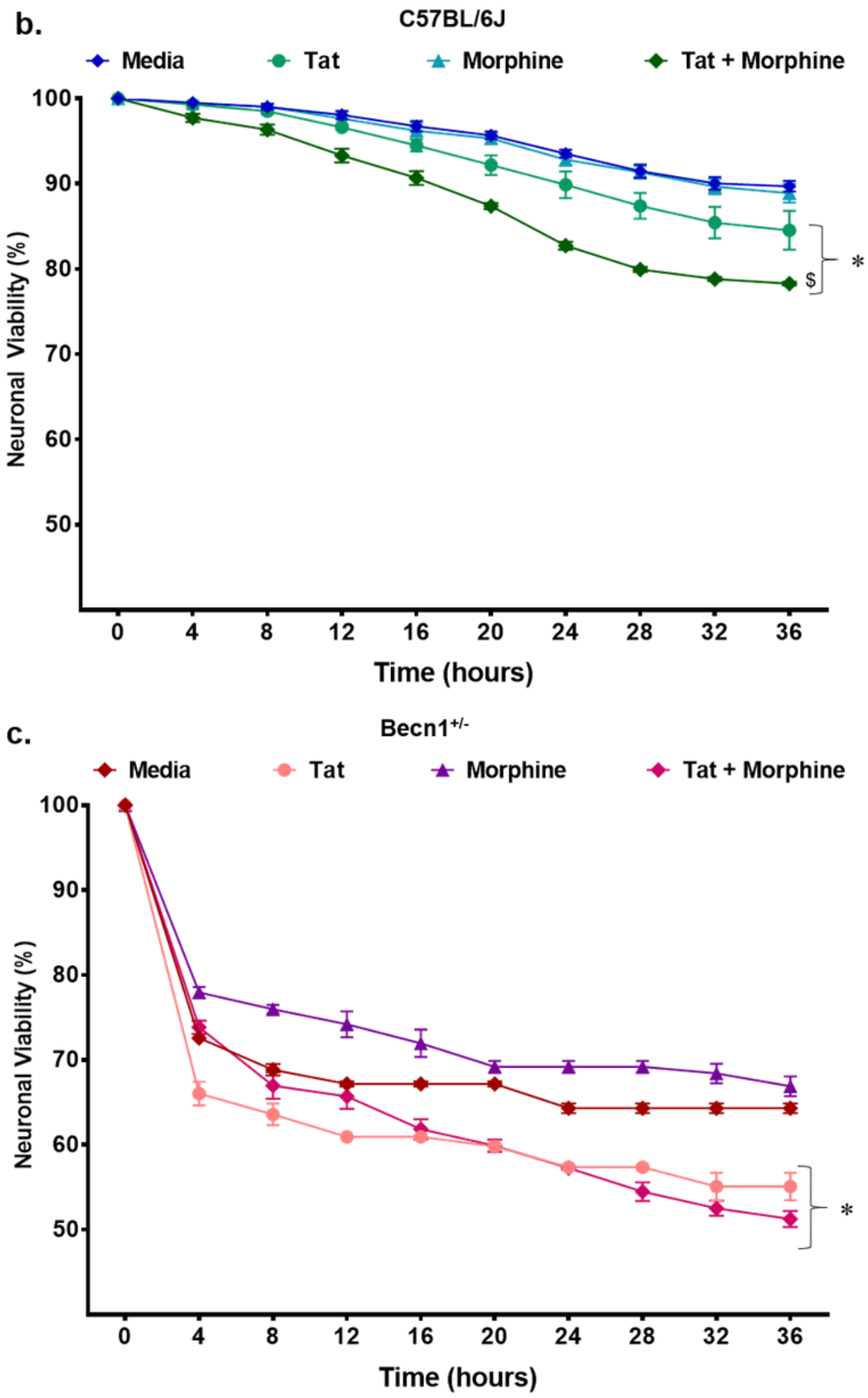
d.
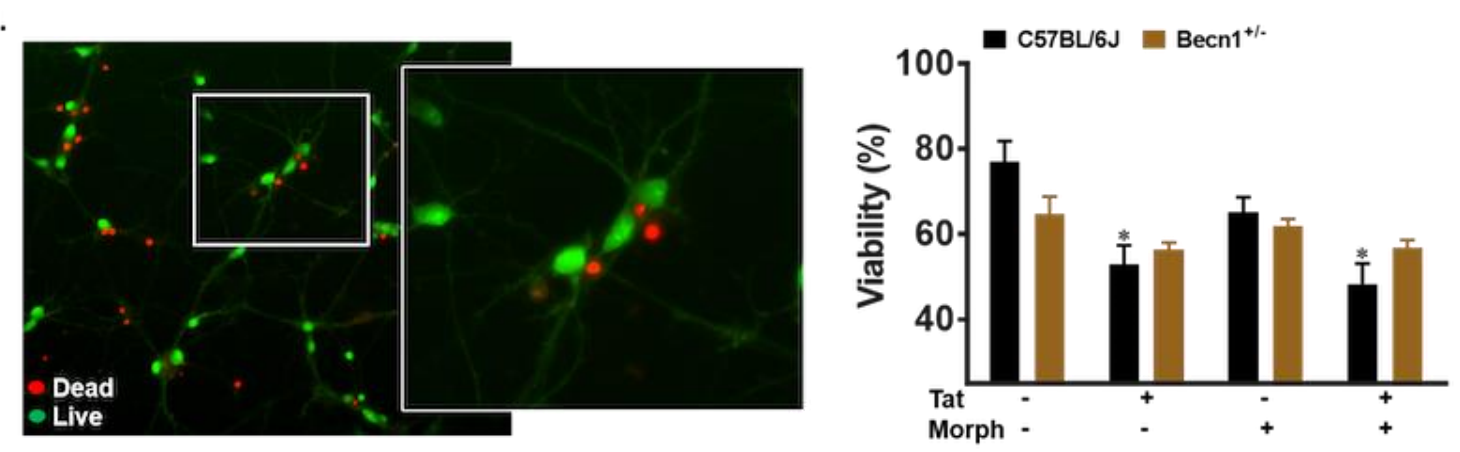

Figure 3.4 Neurotoxic effects of Tat and morphine are more severe in Becn $1^{+/-}$neurons Individual C57BL/6J (b) and Becn1+/- (c) neurons were assessed for survival following Tat and morphine treatment using time-lapse imaging over $36 \mathrm{~h}$. Representative phase contrast images of individual neurons are shown at 20x magnification (a). Red circles depict dead neurons. Becn $1^{+/-}$ neurons were found to be highly susceptible to death independent of treatment. Error bars show the SEM for 3 independent experiments with at least 40 cells per experiment. $\mathrm{P}<0.05$ * vs. Control; $\$$ vs. Tat. RM Two-way ANOVA followed by Tukey's test (treatment effects). Neuronal viability was confirmed using a live/dead fluorescence assay (d). A representative image of dead (red) and live (green) cells acquired at 20x magnification is shown. Viability was manually quantified following the indicated treatments at $36 \mathrm{~h}$. Error bars show SEM for replicates of a representative experiment. $\mathrm{P}<0.05$ * vs. Control; \# vs. C57BL/6J; \$ vs. Tat. Two-way ANOVA followed by Tukey's test.

\subsubsection{Reduction of Beclin 1 expression in glia treated with Tat and morphine lessens indirect toxicity to neurons}

Having surveyed the direct effects of Tat and morphine on neuronal survival, we next questioned how these effects might be modulated in a more complex experimental system which incorporated glial contributions as a closer representation of the brain environment. As previously prefaced, excessive glial activation and dysfunction play a significant role in establishing HIV and Tat-induced neurotoxicity through the production of reactive species, glutamate release, and cytokine/chemokine secretion culminating in neuroinflammation and subsequent neuronal death $[54,86,327,328]$. Using supernatant/conditioned media (CM) from C57BL/6J and Becn1+/- glia treated with Tat and/or morphine, we assessed whether the reduced expression of Beclin 1 in activated glia could ameliorate the neuroinflammation and subsequent indirect toxicity experienced by neurons in the CNS. Based on the poor survival outcomes of Becn1 ${ }^{+-}$neurons, these experiments proceeded with C57BL/6J neurons only. Time-lapse monitoring revealed a statistically significant 
decrease in neuronal survival when given Tat-treated C57BL/6J CM (13\% decrease) and was prominently enhanced when Tat was combined with morphine (30\% decrease) (Figure 3.5b). Morphine-treated C57BL/6J CM did slightly decrease neuronal survival but was not significantly different from what was seen in the untreated $\mathrm{CM}$ ( $7 \%$ decrease). These studies yielded vastly different results when neurons were exposed to CM from Becn $1^{+/}$glia (Figure 3.5c). While the survival of neurons given untreated Becn ${ }^{+/-} \mathrm{CM}$ was comparable to that of untreated C57BL/6J CM at 36 hours (C57BL/6J - 69\%; Becn1+/- 74\%), all other treatments were starkly different than the similarly treated wild type. Tat, morphine, and Tat + morphine treated Becn $1^{+/-} \mathrm{CM}$ all reduced neuronal survival compared to untreated Becn1+- $\mathrm{CM}$, but there were no detectable differences between those 3 treatments $(65 \%, 67 \%$, and $65 \%$ respectively). Viability was confirmed by live/dead assay and trypan blue exclusion which showed similar results (Figure $3.5 \mathrm{~d}$ ). This data supports our hypothesis that targeting the autophagy pathway, specifically Beclin 1, in glial cells can diminish the indirect toxicity and neurodegeneration instigated by Tat and morphine co-exposure in the neuronal microenvironment. 
a.

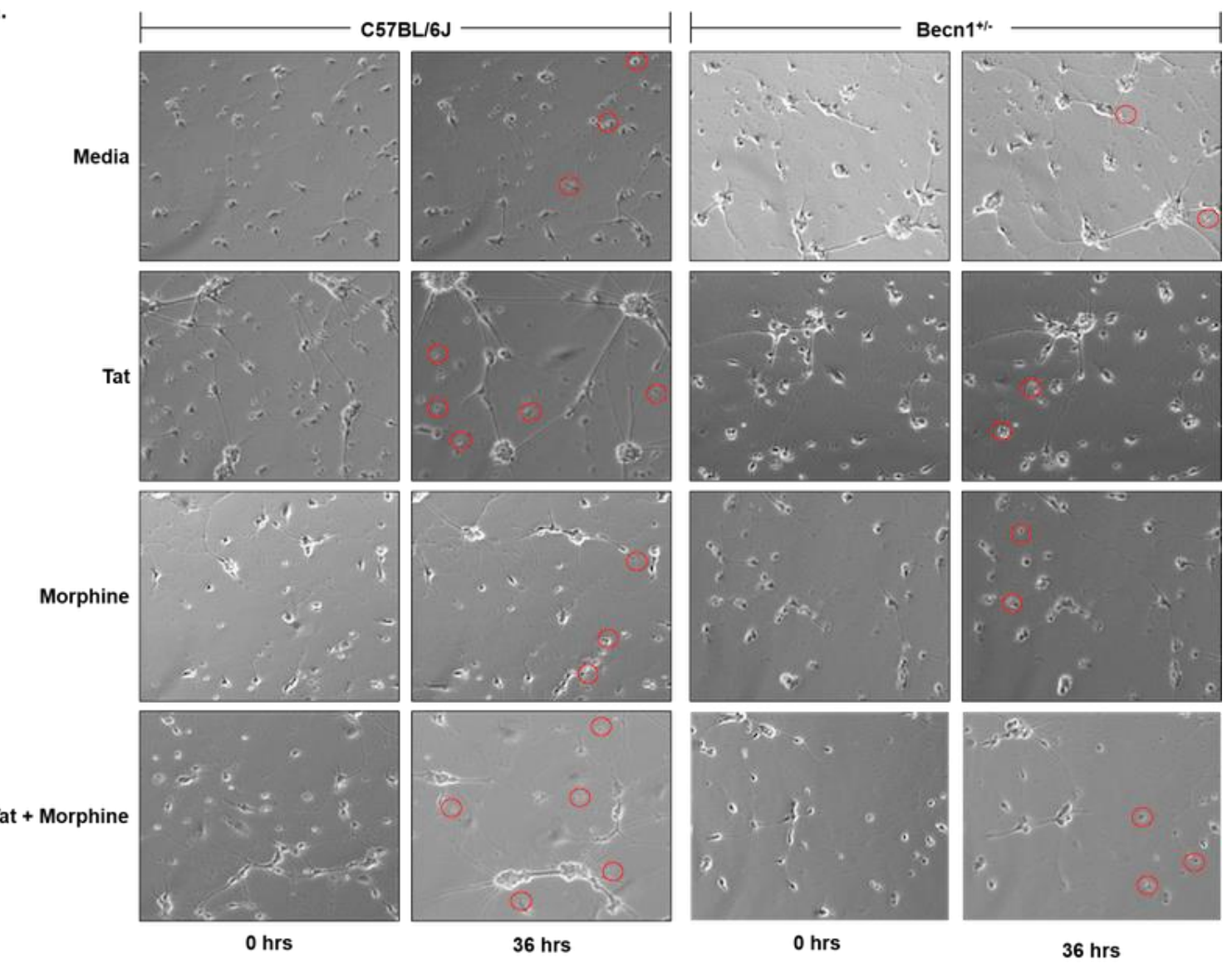



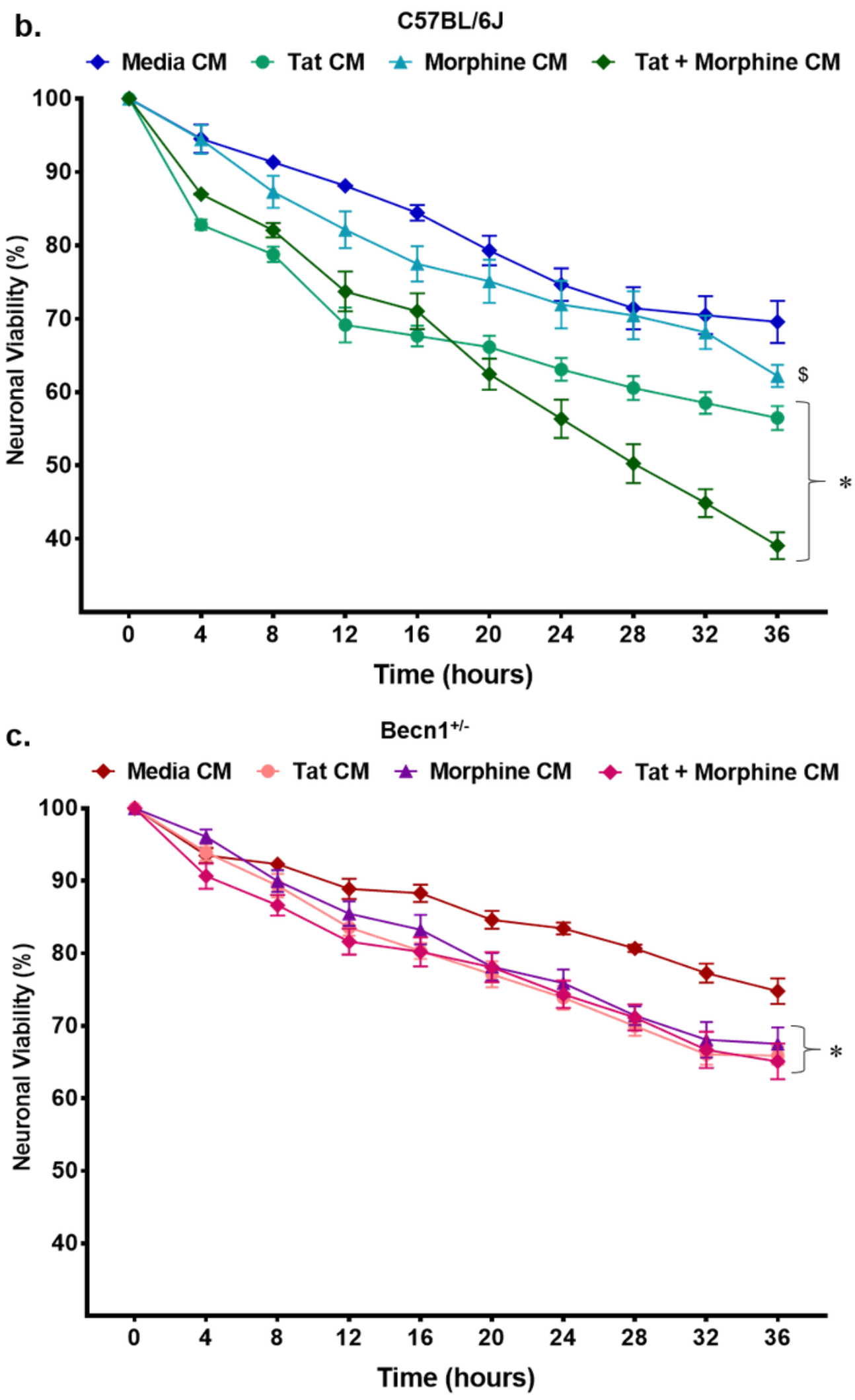
d.
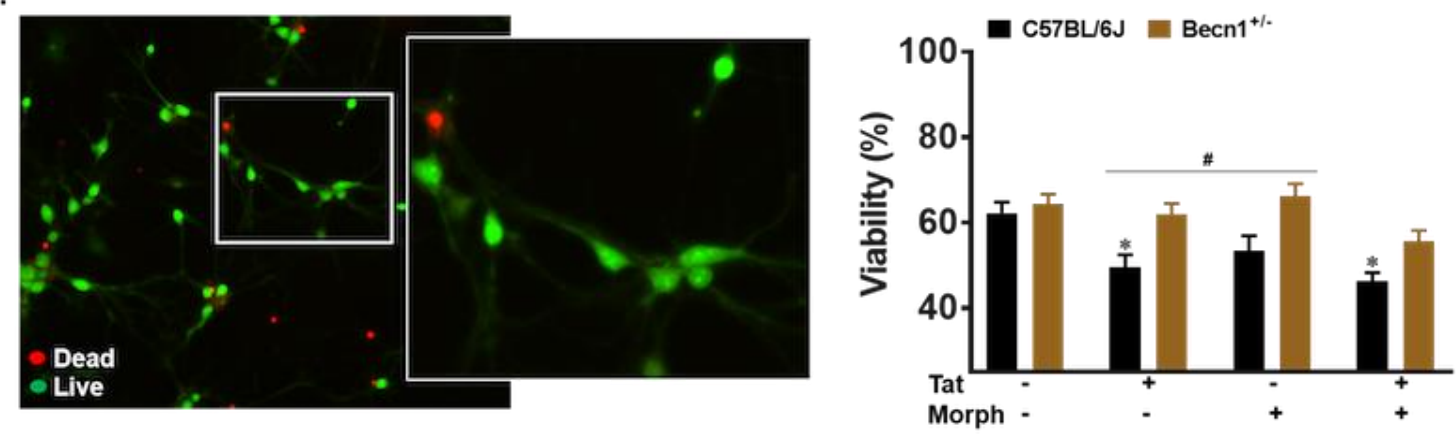

Figure 3.5 Decreased toxicity of neurons exposed to supernatant from Becn $1^{+/-}$glia treated with Tat and morphine

Individual C57BL/6J neurons were assessed for survival following exposure to supernatant from Tat and morphine treated C57BL/6J (b) and Becn $1^{+-}$(c) glia using time-lapse imaging over $36 \mathrm{~h}$. Representative phase contrast images of individual neurons are shown at 20x magnification (a). Red circles depict dead neurons. Error bars show the SEM for 3 independent experiments with at least 30 cells per experiment. $\mathrm{P}<0.05^{*}$ vs. Control; $\$$ vs. Tat. RM Two-way ANOVA followed by Tukey's test (treatment effects). Neuronal viability was confirmed using a live/dead fluorescence assay (d). A representative image of dead (red) and live (green) cells acquired at $20 x$ magnification is shown. Viability was manually quantified following the indicated treatments at $36 \mathrm{~h}$. Error bars show SEM for replicates of a representative experiment. P < 0.05 * vs. Control; \# vs. C57BL/6J; \$ vs. Tat. Two-way ANOVA followed by Tukey's test.

\subsubsection{Beclin 1 associated alterations in growth factor secretion upon Tat and}

\section{morphine exposure}

Based on existing literature and the findings here, glial dysfunction is a clear arbitrator of neurotoxicity and neurodegeneration. As reviewed in Chapter 1, microglia and astrocytes have distinct and crucial functions in moderating the neuronal environment and neuronal homeostasis which are disrupted by HIV and its viral proteins. By that token however, when investigating the effects of HIV on glial function, it is essential to consider the effects duly in terms of gain of function (glial over-activation/production) and loss of function (glial shutdown/under-production). One key task of glial function in support of neuronal homeostasis is the production/secretion of growth/neurotrophic factors. Neurotrophic growth factors are endogenous soluble proteins which regulate a plethora of neuronal actions and functions. These include regulating neuronal survival, growth, morphological plasticity/synthesis, and protein synthesis for differentiated neurons [329, 330]. To evaluate the changes to soluble factor support potentially experienced by neurons due 
glial Tat and morphine exposure, we measured growth factor secretion from C57BL/6J and Becn $1^{+/}$supernatant using the RayBio ${ }^{\circ}$ C-Series Mouse Growth Factor Array. This array probed for the secretion of 30 growth factors, where from that 30 , those with significant fold changes are included in Table 3.1. From that list, several growth factors have known associations to HIV-induced dysfunction and/or regulating neuronal health (Figure 3.6). Basic fibroblast growth factor (bFGF), insulin-like growth factor 1 (IGF-1), macrophage colony-stimulating factor (M-CSF), and platelet-derived growth factor (PDGF) are all mitogenic growth factors which are secreted by cells to stimulate growth and differentiation, maintain cell survival, recruit circulating cells, and regulate the cellular microenvironment. Just as in the periphery, these functions are executed within the brain by the resident microglia, astrocyte, and neuronal cells in response to an initial stimulus. bFGF, for example, is secreted by astrocytes where, in addition to its promotion of cell survival and proliferation, it has been reported to stimulate the neurite expansion and branching of neurons [331, 332]. IGF-1 is primarily associated with astrocytes and neuron; when bound to its receptor IGF1R, triggers pro-survival signaling cascades, enhances nerve growth, and promotes synaptic plasticity (the process by which changes in synaptic activity strengthen or weaken synapse strength) [333-335]. M-CSF regulates myeloid cell activation and growth in the periphery but in the CNS, where it is produced by glial cells, promotes the survival and proliferation of microglia as well as regulates their activity [336, 337]. In addition, some studies report neuroprotective actions of M-CSF in neurodegenerative disease models such as the AD mouse model [338]. PDGF is made by platelets and regulates cell growth and division but can also be made by astrocytes and is required for normal early development. Here we were able to detect significant differences in bFGF, IGF-1, and M-CSF between the wild type and mutant strains when exposed to Tat and morphine alone or in combination (Figure 3.6). Becn $1^{+/}$glia treated 
with Tat and morphine both showed the highest levels of these neurotrophic growth factors with the difference between it and similarly treated C57BL/6J glia being quite substantial. This may represent Becn1+/- glia more efficiently responding to Tat and morphine exposure and subsequent neuronal damage through the secretion of mitogenic prosurvival neurotrophic growth factors. As such, targeting Beclin 1 expression in glia may be considered beneficial to neuronal survival. Separately, the differences in PDGF-AA were more subtle and not statistically significant (Figure 3.6d). Others have described the neuroprotective potential of a PDGF isoform, PDGF-BB, which was shown to attenuate Tat and morphine-induced damage to human neurons; however, in our studies we found no detectable levels of PDGF-BB in either C57BL/6J or Becn1+/- supernatant [339]. In summary, this data establishes a role of Beclin 1 in regulating glial growth factor production and highlights a different, potentially overlooked, aspect of glial function and response which may contribute to enhancement of neuronal survival.

Table 3.1 Soluble growth factor release in C57BL/6J and Becn1 ${ }^{+/-}$glia treated with Tat and morphine

\begin{tabular}{|c|c|c|c|c|c|c|}
\cline { 2 - 7 } & \multicolumn{2}{|c|}{ Tat } & \multicolumn{2}{c|}{ Morphine } & \multicolumn{2}{c|}{ Tat + Morphine } \\
\hline $\begin{array}{c}\text { Growth } \\
\text { Factor }\end{array}$ & C57BL/6J & Becn1 ${ }^{+/ \cdot}$ & C57BL/6J & Becn1 $1^{+/ \cdot}$ & C57BL/6J & Becn1 $1^{+/ \cdot}$ \\
\hline bFGF & $75.05 \pm 20.5$ & $114.14 \pm 13.6$ & $42.24 \pm 1.1$ & $148.30 \pm 16.5$ & $174.01 \pm 37.6$ & $379.65 \pm 33.7$ \\
\hline IGF-1 & $97.56 \pm 14.3$ & $77.91 \pm 26.4$ & $45.61 \pm 2.0$ & $87.44 \pm 2.5$ & $48.40 \pm 7.2$ & $354.75 \pm 53.6$ \\
\hline IGF-2 & $110.98 \pm 23.6$ & $92.25 \pm 28.8$ & $66.14 \pm 11.8$ & $21.55 \pm 8.1$ & $94.09 \pm 7.3$ & $175.90 \pm 35.8$ \\
\hline IGFBP-3 & $80.78 \pm 15.9$ & $62.13 \pm 9.0$ & $72.09 \pm 10.4$ & $110.67 \pm 5.28$ & $72.96 \pm 7.5$ & $264.36 \pm 11.9$ \\
\hline IGFBP-5 & $85.53 \pm 15.8$ & $65.00 \pm 35.5$ & $52.52 \pm 19.4$ & $47.38 \pm 27.1$ & $69.87 \pm 1.9$ & $333.83 \pm 33.3$ \\
\hline IGFBP-6 & $64.05 \pm 5.1$ & $101.61 \pm 24.7$ & $43.63 \pm 11.7$ & $39.15 \pm 26.8$ & $93.96 \pm 21.4$ & $201.96 \pm 6.6$ \\
\hline M-CSF & $82.52 \pm 12.3$ & $36.00 \pm 5.6$ & $85.46 \pm 7.9$ & $49.51 \pm 8.6$ & $54.47 \pm 6.1$ & $174.45 \pm 9.6$ \\
\hline PDGF-AA & $119.08 \pm 19.4$ & $95.42 \pm 5.5$ & $102.82 \pm 20.6$ & $82.86 \pm 10.9$ & $140.34 \pm 4.2$ & $121.60 \pm 1.2$ \\
\hline PLGF & $68.54 \pm 13.9$ & $74.49 \pm 3.4$ & $61.23 \pm 19.8$ & $48.33 \pm 16.7$ & $101.67 \pm 21.1$ & $87.94 \pm 10.1$ \\
\hline VEGF-A & $70.39 \pm 9.7$ & $62.02 \pm 10.9$ & $100.21 \pm 31.2$ & $31.77 \pm 3.5$ & $110.60 \pm 3.2$ & $162.02 \pm 13.2$ \\
\hline
\end{tabular}



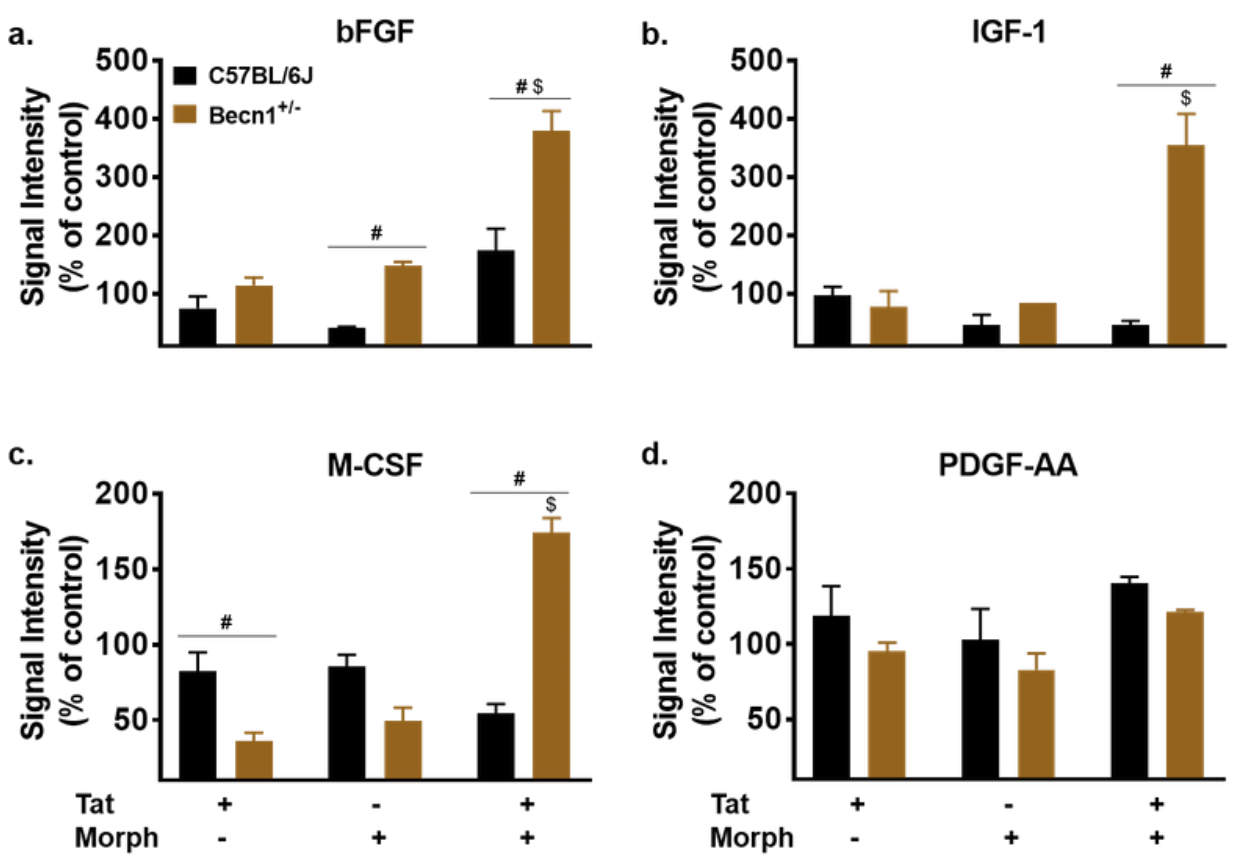

Figure 3.6 Tat and morphine treatment in Becn $1^{+/-}$glia increases secretion of neurotrophic growth factors

Selected growth factors showed significant modulations in secretion from Tat \pm morphine exposure. $\mathrm{P}<0.05$ * vs. Control; \# vs. C57BL/6J; \$ vs. Tat. Two-way ANOVA followed by Tukey's test.

\section{$\underline{3.4 \text { Discussion }}$}

In this chapter, we examined the role of the autophagy protein Beclin 1 in mediating direct and indirect neuronal toxicity upon Tat and morphine co-exposure. As in the previous chapter, to do this we make use of mice possessing a monoallelic deletion of the Becn1 gene and embryonically-derived primary neurons isolated from them. Neuronal autophagy is a highly sensitive process of which aberrant activation or inhibition frequently cause dysfunction leading to disease. These long-lived cells are post-mitotic and do not have the luxury of cell division to serve as a contingency under pathological conditions where damaged and dysfunctional organelles often accumulate. As such, neurons are heavily reliant on the proteasome and lysosome systems for the maintenance of neuronal function $[158,322,340]$. Tat exposure in neurons has been shown to greatly affect cellular 
autophagy, although to different extents. Hui et al found Tat ${ }_{1-72}$ to cause the accumulation of abnormally large endolysosomes with elevated $\mathrm{pH}$ paired with decreased expression of autophagy proteins LC3, ATG5, and increased p62/SQSTM1, implying an inhibition of autophagy in hippocampal neurons [248]. Another study by Fields et al reported that Tat ${ }_{1}$. ${ }_{86}$ also caused a reduction of autophagy in cortical neuronal neurons also with abnormal accumulation of autophagosomes; however, though somewhat contradicting, found that Tat could reduce the autophagic blockade effects of the V-ATPase inhibitor bafilomycin A1 which suggests that Tat promotes autophagosome and lysosome fusion in neurons [191]. In studies of HIV exposure, one study reported inhibition of autophagy in cortical neurons exposed to supernatant from SIV infected microglia with decreased LC3 and increased p62/SQSTM1 which could be rescued by autophagy induction using rapamycin [189], whereas two separate studies found increased expression of autophagy proteins Beclin 1, ATG5, ATG7, and LC3 in the post-mortem tissues of HIVE patients $[185,187]$. Taken together, it is apparent that the effects of HIV and the viral protein Tat on neuronal autophagy are complex.

In these studies, we first investigated the direct effects of Tat and morphine co-exposure on neuronal function, structure, and survival to ascertain the part played by Beclin 1 in mediating these effects. Neuronal function is largely dependent on the various signaling pathways regulated by the levels of intracellular ions such as calcium. Excessive increases in intracellular calcium have been documented to be triggered by Tat interacting with/entering cells through receptors and surface proteins such as CXCR4, NMDAR, heparan sulfate proteoglycans, and low-density lipoprotein (LDL) receptor-related proteins, and often lead to neuronal cell death [319, 341-344]. The glutaminergic/ionotropic NMDARs may be activated by Tat leading to ionic imbalances 
and excitotoxicity [273, 345]. Here we were able to detect Tat induced increases in intracellular calcium in C57BL/6J neurons as has been previously reported [251, 260]. This was further increased by the addition of morphine which was previously demonstrated by Fitting et al to converge on MORs and exacerbate calcium accumulation by mobilizing intracellular stores [316]. While these effects on C57BL/6J neurons were to be expected, we had not expected the heightened effects of Tat and morphine on Becn $1^{+/-}$ neurons compared to the wild type counterparts. Although we did not observe any additive or synergistic effects with Tat and morphine co-exposure in the Becn $1^{+-}$neurons, both treatments of Tat alone and in combination with morphine surpassed that which was seen in the C57BL/6J (Figure 3.2). From this it can be suggested that Beclin 1 and the autophagy pathway may have direct ties to mediating neuronal excitotoxicity. One study on traumatic brain injury found that glutamate could induce excitotoxity in cortical neurons through $\mathrm{N}$-methyl D-aspartate receptor subtype 2B (NR2B) while in parallel activating the autophagy pathway and increasing expression autophagy proteins like Beclin 1 and LC3. When NRB2 was pharmacologically antagonized, this prevented both glutamate toxicity and autophagy induction [346]. A separate study found that NMDA-induced autophagy in cortical neurons as well as dendrimer-mediated siRNA delivery against Beclin1 was able to potentiate NMDA toxicity [347]. This may be correlated with studies of Tat which also showed that exposure can cause activation of neuronal autophagy [191]. These studies and the calcium data presented in this chapter suggest that Beclin 1 may be part of a defense mechanism against excitotoxicity and further establishes the neuroprotective role of autophagy in neurons within the context of Tat and morphine induced excitotoxity. Despite this, the question of how exactly autophagy proteins and structures interact with NMDARs to prevent calcium overload still stands. This may be through the triggering of pro-survival proteins such as BCL-2 or p53, mitochondrial membrane destabilization and 
outer membrane puncture, targeting of $\mathrm{Ca}^{2+}$ or V-ATPases, or through phosphorylation signaling cascades involving development/cell division proteins such as CDK5 and Wnt/ $\beta$ Catenin all of which have been associated with induction of neuronal autophagy and glutamate excitotoxicity in models of ischemia or HAND [348-353]. Ultimately, more research into the exact mechanisms are still required.

The neuroprotective functions of Beclin1 and autophagy was also illustrated by our findings in neurite beading (Figure 3.3). Here we saw that while Tat, alone and in combination with morphine, did increase neuronal beading as has been shown in previous studies $[187,316]$, this was significantly heightened in Becn $1^{+/-}$neurons. Synaptodendritic injury is comprised of structural changes such as dendritic pruning and focal swelling displayed as varicosities or beads which disrupt communication at the synapse and axonal transport [321]. The development of these beads along the dendrites is considered a marker for neuronal injury and excitotoxic stress which links beading with intracellular ion flux. Functionally, when NMDARs are activated, the channel opens and allows for the influx of not only $\mathrm{Ca}^{2+}$ ions but also $\mathrm{Na}^{+}$at a higher rate [354]. Elevated intracellular $\mathrm{Na}^{+}$ not only accompanies $\mathrm{Ca}^{2+}$ but is also able to modulate NMDAR gating and expression which exacerbates ionic imbalances [355-357]. It has been suggested this excess of $\left[\mathrm{Na}^{+}\right]_{\mathrm{i}}$ and dysregulated $\left[\mathrm{Ca}^{2+}\right]_{i}$ causes the structural manifestation of neurite beading through not only excitotoxic influx on ions, but also the accompanied alterations in cellular energetics and ATP generation which are dependent on $\mathrm{Na}^{+} / \mathrm{K}^{+}$ATPase activity and is also associated with autophagic cell death or autosis [356, 358, 359]. Further, a recent study in HIV-infected macrophages found that a Tat-Beclin 1 peptide was able to induce this form of autophagic cell death mediated by $\mathrm{Na}^{+} / \mathrm{K}^{+}$ATPase activation [360]. Taken together, this may explain our beading results shown in Figure 3.3 from treated Becn $1^{+/-}$ 
neurons and correlates it with our findings from Tat and morphine induced $\mathrm{Ca}^{2+}$ accumulation in Becn1+- neurons shown in Figure 3.2. It should be noted that dendritic beading is a reversible occurrence so other assessments of neuron morphological damage can also be utilized in future experiments such as evaluating spine density, dendritic length and branching which may correlate to synaptic damage [361].

The importance of Beclin 1 and autophagy in regulating neuronal health was further confirmed when C57BL/6J and Becn1+-- neuronal survival was monitored over 36-hours by time-lapse imaging following Tat and/or morphine treatment. Previous studies have examined the effects of Tat and morphine alone and in combination on neuronal viability and have found the exacerbating effects to striatal neurons through apoptotic pathways [101]. We similarly found that $100 \mathrm{nM}$ Tat treatment caused mild neuronal toxicity in C57BL/6J neurons which was augmented by morphine co-exposure (Figure 3.4b); however, this toxicity was magnified in similarly treated Becn1 ${ }^{+-}$neurons (Figure 3.4c). This reiterates the importance of Beclin 1 in neurons and corroborates the neurotoxicity suggested by the $\left[\mathrm{Ca}^{2+}\right]_{i}$ and neurite beading assessments (Figure 3.2 and 3.3). It should be noted that time-lapse assessment of viability monitors death over a duration of time but does not incorporate assessment of apoptosis. As discussed in chapter 1.7.2, Beclin 1 and the autophagy process is tightly coordinated with apoptosis. Based on this, in future studies it is essential to distinguish the mechanisms of neuronal death, whether through apoptosis, autophagic cell death, or other contributing mechanisms which can be done using methods such as TUNEL and annexin V staining in combination with inducers and inhibitors of autophagy and apoptosis [362, 363]. Studies have demonstrated the neuroprotective functions of Beclin 1 in neurodegenerative diseases such as AD and PD [364, 365]. One study by McKnight et al highlighted the importance of Beclin 1 when they 
showed how in cerebellar Purkinje, cortical, and hippocampal neurons where the protein is conditionally deleted, neurons experience rapid degeneration and increased mouse mortality as early 6 weeks with all mice dying by the age of 4 months [262]. This is understandable since complete Beclin 1 deletion $\left(B e c n 1^{-1}\right.$ ) is shown to be embryonically lethal whereas the Becn1+/- mice used here have been shown to live up to 18 months, although they do exhibit late onset lung carcinomas, hepatocellular carcinomas and lymphomas $[136,137]$. However, in the study by Pickford et al which uses the same heterozygous Becn $1^{+/}$-mutant mice used in our study, neuronal Beclin 1 deficiency also resulted in synaptodendritic degeneration, abnormal lysosomes with electron dense granules, and reduced MAP-2 and synaptophysin (neuronal/synaptic markers) immunoreactivity in vivo, all of which were enhanced when Becn $1^{+/}$mice were crossed with the $\mathrm{APP}^{+}$transgenic mouse, an $\mathrm{AD}$ mouse model [364]. Taken together, this establishes the compromised state of Becn $1^{+/}$striatal neurons as determined by $\left[\mathrm{Ca}^{2+}\right]_{\mathrm{i}}$ accumulation and increased dendritic damage which is further exacerbated by Tat and morphine exposure, ultimately leading to rapid neuronal loss.

As referenced earlier, the neuronal damage mediated by HIV and its viral proteins does occur independently without the contributions of the glial cells in the CNS environment, especially in the context of drug abuse. Glia-mediated neuroinflammation through the secretion of cytokines and chemokines is noted as a key facet of HAND, in addition to metabolic dysfunction, impaired neurotrophic activity, reactive gliosis, and oxidative stress all of which inevitably prompt neuronal injury and loss $[60,106,312,366]$. In this study we examined the effects of glial conditioned media on neuronal survival as assessed by timelapse monitoring. The differences in C57BL/6J neuronal survival between the experiments of direct exposure to Tat and morphine and exposure to the glial secretions as a result of 
Tat and morphine exposure were stark (Figures $3.4 \mathrm{~b}$ and $3.5 \mathrm{~b}$ ). Neuronal viability was dramatically impaired when exposed to the $\mathrm{CM}$ from Tat or Tat + morphine treated C57BL/6J glia compared to the when the neurons were exposed directly. This coincides with existing literature which underscore the importance of glia in eliciting neuronal death upon Tat exposure [54, 56, 66, 87, 240]. This is particularly important in the context of Tat and morphine co-exposure [86, 87, 100, 106, 243, 261, 264, 284]. Zou et al for example demonstrated the importance of not only glia in mediating the neurodegenerative effects of Tat and morphine combined exposure, but also the necessity of glial MOR expression to potentiate these effects [102]. The study found that glial conditioned media enhanced the synergistic toxicity to neurons upon Tat and morphine exposure of which synergy was dependent on the presence of glial, but not neuronal, MOR expression as determined by using MOR 1 - knockout mice. These potentiating effects were even greater when neurons were placed into direct contact with glia, emphasizing the importance of soluble factor secretion in augmenting toxicity. Other studies which exposed neurons to the conditioned media of either SIV/HIV-infected microglia or Tat-treated glia also showed neurodegeneration in the forms of dendritic pruning and cell death which may be attributed to dysfunctional neuronal autophagy $[187,189]$. When we examined the effects of Becn $1^{+/-}$ $\mathrm{CM}$ on neuronal survival, we observed an attenuation of Tat and morphine-induced toxicity compared to that of $\mathrm{C} 57 \mathrm{BL} / 6 \mathrm{~J} \mathrm{CM}$. Based on the data presented in the previous chapter on inflammatory molecule secretion, reduction of Beclin 1 expression in glia is able to partially abrogate inflammation induced by Tat alone or in combination with morphine (Figure 2.8) which we have also seen with HIV-infected microglia and astrocytes [192, 246]. In addition, although glutamate buffering was not shown here, our previous studies in human astrocytes demonstrated that silencing Beclin 1 by siRNA can also rescue impaired glutamate uptake caused by HIV infection which contributes to excitotoxicity in 
neurons [246]. This supports the notion that targeting glial, but not neuronal, autophagy through Beclin 1 can help to attenuate Tat and morphine induced neurotoxicity. Our analysis of glial growth factor secretion following Tat and/or morphine exposure may further substantiate this concept. Tat and morphine were found to cause significant elevation of growth factors such as bFGF, IGF1, and M-CSF in Becn1/- glia (Figure 3.6). While this may seem counterintuitive, studies have shown the upregulation of growth factors in neurodegenerative diseases such as AD, PD, and HAND [367, 368]. This may possibly be explained by glial efforts to counteract the neuronal damage such as dendritic beading being caused by toxic exposure in the brain [366]. bFGF for example has been documented to be significantly raised in reactive astrocyte dense areas of the brains of patients with AIDS dementia complex [367]. It was hypothesized that glia secrete neurotrophic factors to stimulate pro-survival cues in the ailing neurons but ultimately lose that battle. By that note, it is possible, through some yet to be determined mechanism, for Tat and morphine exposure within a Beclin 1-deficient host cell to trigger sufficient production of neurotrophic factors (while also limiting inflammatory molecule secretion) and activate neuroprotective mechanisms to prevent further, and possibly reverse, neuronal damage. The neuroprotective qualities of neurotrophic growth factors in response to oxidants or excitotoxicity have been documented and are considered in potential treatments for neurodegenerative disease [330, 339, 369-371]. As such, the capability of Beclin 1 to modulate not only Tat and morphine induced inflammation, but also neuroprotective growth factor secretion poses an attractive target in the design of future therapeutic interventions for HAND in the context of drug abuse and prevent further neuronal damage. Currently, further studies are ongoing to delve further into the mechanisms of autophagic cell death which are mediated by Tat and morphine exposure. 
CHAPTER 4: CONTRIBUTIONS OF BECLIN 1 TO TAT AND OPIOID NEUROPATHOLOGY AND MOTOR IMPAIRMENT WITHIN THE MOUSE STRIATUM

\section{$\underline{4.1 \text { Introduction }}$}

The umbrella term HAND encompasses a wide breadth of cognitive, motor, and behavioral abnormalities associated with CNS presence of HIV [52]. Currently, diagnosis of HAND is somewhat challenging since clinical features are no longer as pronounced as in the precART era. Initially, the AIDS Task Force of the American Academy of Neurology (AAN) founded the criteria by which the neurological stages of HIV are defined; however, these were revised to account to the lesser pronounced and more mild forms of neurological impairment [372]. The updated criteria of HAND assess neuropsychometric performance (NP) which includes attention information processing, language, memory (learning and recall), sensory perceptual skills, simple motor skills, and complex perceptual motor skills where deficits may appear in ANI or later progress from it. However, initial early identification is reliant on self-reporting which may incorporate bias [373]. Neuroimaging is also increasingly being utilized to monitor metabolic, structural, and functional changes within HAND patients [374].

Advancements in antiretroviral treatments have greatly changed the landscape of HIV and neurological decline. Adherence to cART significantly reduced both the incidence and rate of mortality due to HAD, the most severe manifestation of HAND. In spite of these therapeutic successes, the rates of the lesser forms of HAND, MND and ANI, have gradually been on the rise [375]. These two classifications also bring their own, more subtle, clinical manifestations which are still prevalent in the post-cART era and hinder the day to day quality of life in HIV infected persons. There are a number of contributing 
factors which may explain the continued prevalence of HAND clinical characteristics including variable antiretroviral penetrance to the brain, antiretroviral toxicity, and continuous exposure to secreted viral proteins, all of which are compounded by the increased life span of HIV infected individuals [376, 377].

Although HIV has the capability of affecting multiple brain regions, pathology associated with infection has been most prominent in the basal ganglia, with the frontal cortex and hippocampus also displaying significant levels of viral burden [378-380]. Coinciding with these locations of high viral load, patients with HIV often presented with the inability to complete tasks, delayed speech output, impaired fine motor skills, and unsteady gait [13]. In addition, patient brain histopathology indicated cortical atrophy, formation of multinucleated giant cells, and immune cell infiltration leading to encephalitis [381, 382]. This was also found to be more rapid and severe in patients who were IDU, likely because virus laden areas like the basal ganglia are also highly rich in opioid receptor expression $[380,383]$. Focusing on the basal ganglia, the motor disorders associated with HIV include Parkinsonism in more severe occurrences. In more mild cases of impairment, reports include isolated eye movement impairment, bradykinesia, impaired facial expression, and tremors [384-390]. In general, these clinical demonstrations in HAND are attributed to abnormalities within the basal ganglia, particularly the striatum upon virus or viral protein exposure. In vitro/vivo studies have reported neuronal loss, metabolic dysfunction (dopaminergic pathways), astrogliosis, and inflammation; however, there is still much to be understood in the regulation and eventual prospective treatments for this pathology. In this chapter, we first sought to recapitulate the motor deficits seen in HAND patients using an animal model treated with the viral protein Tat, which has been shown to induce brain pathology, and examine any potential additional dysfunction with morphine co-exposure. 
In parallel, we also used the Becn $1^{+-}$mouse as a potential "therapeutic" model to determine whether any observable behavioral deficits and accompanying pathology of Tat and morphine co-exposure were modulated by the autophagy pathway, and if Beclin 1 intervention can rescue this phenomenon.

\subsection{Materials and Methods}

The guidelines of the National Institutes of Health Guide for the Care and Use of Laboratory Animals were followed, and the Florida International University Institutional Animal Care and Use Committee approved all animal experimental protocols for these studies.

\subsubsection{Animals}

C57BL/6J and Becn1+-- mice were obtained as described in Chapter 2.2.1 and bred. Mixed litters were separated after weaning for later age-matched experiments. Only adult male C57BL/6J and Becn1+/- mice age 3-5 months and weighing approximately 25-30g were used for these experiments. All mice were housed 3-5 per cage and were maintained in a temperature- and humidity-controlled room on a 12:12 h light/dark cycle with lights off at 6:00 pm with ad libitum access to food and water. Mice from each strain were randomly divided into 4 treatment groups: (a) intrastriatal vehicle + placebo implant, (b) intrastriatal vehicle + morphine implant, $(\mathrm{c})$ intrastriatal Tat + placebo implant, $(\mathrm{d})$ intrastriatal Tat + morphine implant (Figure 4.1). 


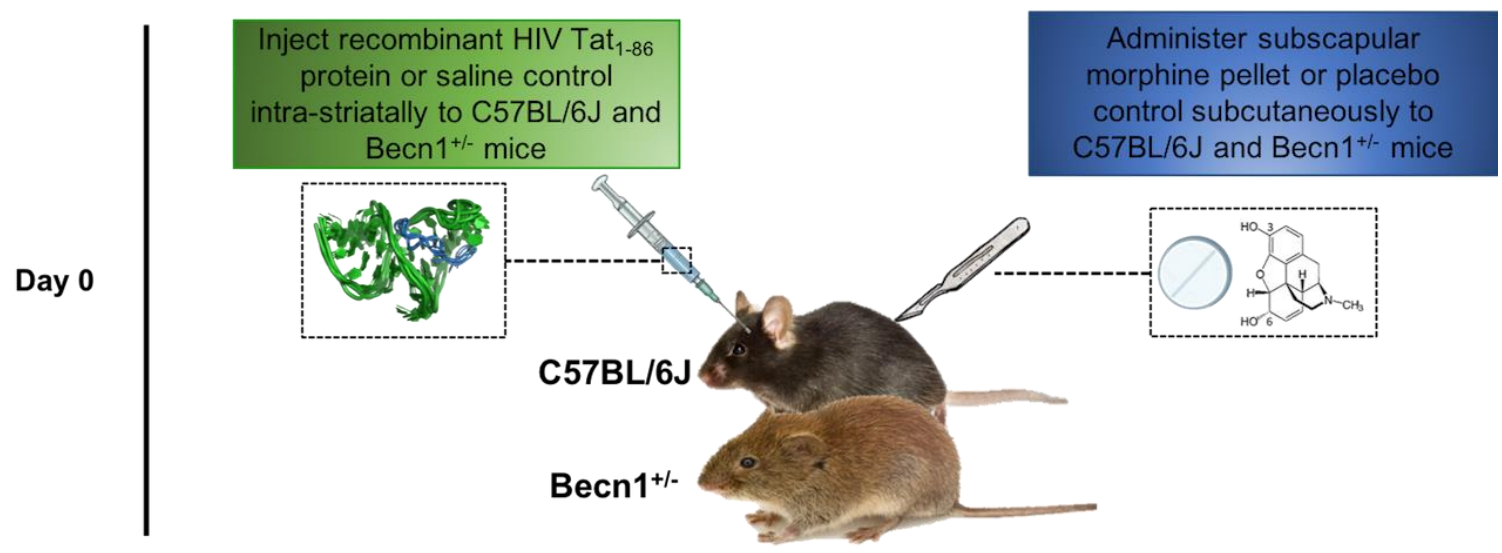

Figure 4.1 Experimental design and surgical plan of Tat and morphine exposure

Under anesthesia, 10ug (in $10 \mu \mathrm{l}$ ) of recombinant HIV Tat $1-86$ protein was injected into the striatum of C57BL/6J and Becn $1^{+/-}$mice. Subsequently, a $25 \mathrm{mg}$ morphine pellet was implanted subcutaneously beneath the scapula. Saline was used as a control for Tat. A placebo pellet was used as a control for morphine.

\subsubsection{Chemicals}

Morphine sulfate $(25 \mathrm{mg})$ and placebo pelleted implants were obtained from the National Institute on Drug Abuse (NIDA, Drug Supply System). Morphine pellets were continuous time-release pellets and were used to continuously deliver drugs for 5 days (approximately $5 \mathrm{mg} /$ day). Recombinant HIV Tat $1-86$ IIIB was purchased from ImmunoDiagnostics.

\subsubsection{Surgical Manipulation}

Mouse surgery was performed as using previously established protocols [244]. Mice were anesthetized by $2.5 \%$ isoflurane and subsequently placed in a mouse stereotaxic apparatus (Stoelting Co.) Under aseptic conditions, mice were administered sterile saline (vehicle control) or $10 \mu \mathrm{g}$ of Tat in a volume of $10 \mu \mathrm{L}$ that was injected over 5 minutes at a rate of $2 \mu \mathrm{L} / \mathrm{min}$ into the striatum using a 33-gauge syringe (Hamilton Co.) attached to an automated infusion pump (Hamilton Co). Striatal injections were made at the coordinates $\mathrm{AP}=+0.7 \mathrm{~mm}, \mathrm{ML}=2.0 \mathrm{~mm}$, and $\mathrm{DV}=-4.0 \mathrm{~mm}$ from the bregma (Figure 
4.2) [242]. After 1 minute of incubation to allow tissue to return to original conformation and prevent backflow, the needle was slowly withdrawn. While still under anesthesia and maintaining aseptic conditions, subscapular skin was lifted, and a $3 \mathrm{~mm}$ incision made creating a subcutaneous pocket and the placebo or morphine pellet was inserted. Mice not given morphine were administered $0.25 \%$ bupivacaine $(6 \mathrm{mg} / \mathrm{kg})$ as local anesthesia before final skin closure using Vetbond surgical glue. Mice recovered on a heating pad $\left(28^{\circ} \mathrm{C}\right)$ until consciousness was regained and normal activity continued before being returned to cages. All animals were carefully monitored daily for signs of distress, weight loss, or changes in behavior.
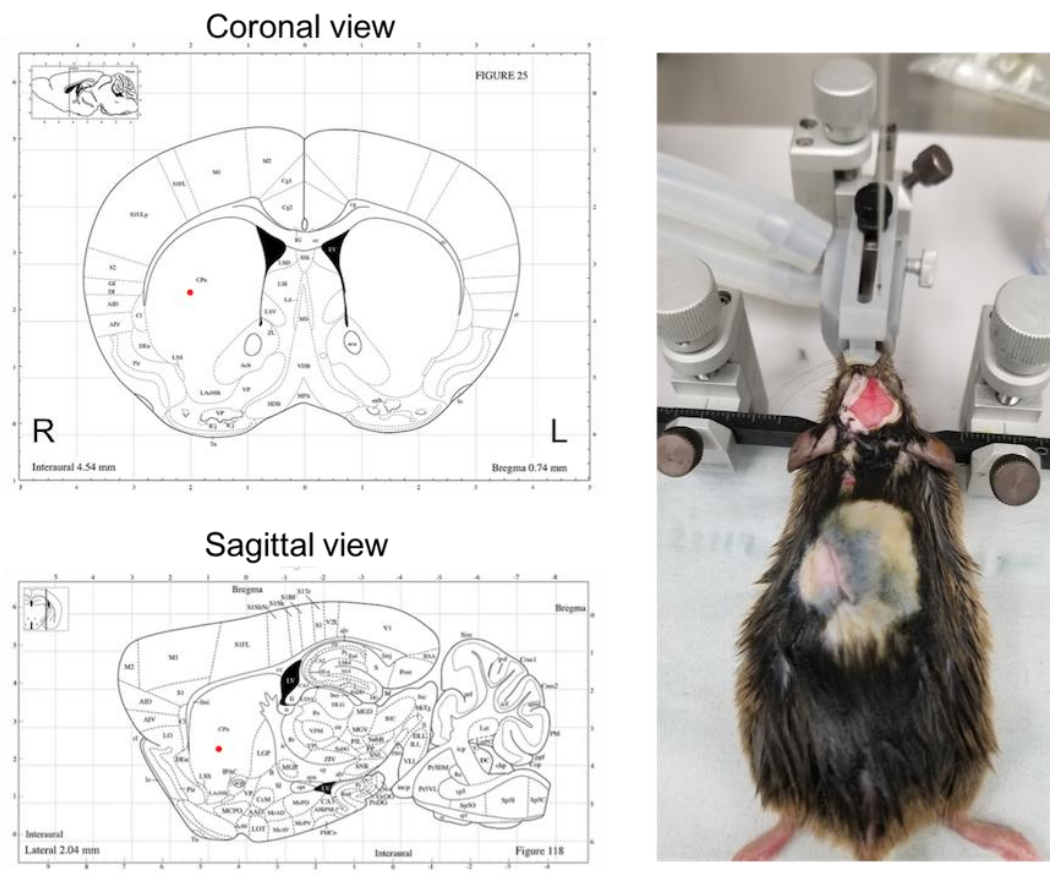

Figure 4.2 Stereotaxic coordinates and surgical example

Striatum was located by stereotaxic reference $(A P=+0.7 \mathrm{~mm}, \mathrm{ML}=2.0 \mathrm{~mm}$, and $\mathrm{DV}=-4.0 \mathrm{~mm})$ relative to the bregma. Injections were done in the caudate/putamen area. 


\subsubsection{Behavioral Assays}

After 2- and 5-days post-surgery, behavioral tests assessing motor skill were performed (Figure 4.3). On the day of behavior testing, mice were habituated in the testing room for one hour prior to assessment. Mice were allowed 3 attempts for each behavior test (unless otherwise stated) with a 5-minute time interval between trials and the best score recorded. Each animal was given a 10-minute rest period between behavior tests. Any mice perceived as being in distress or incapable of performing behavioral tasks were excluded from study results. All mice performed all of the behavior tests.

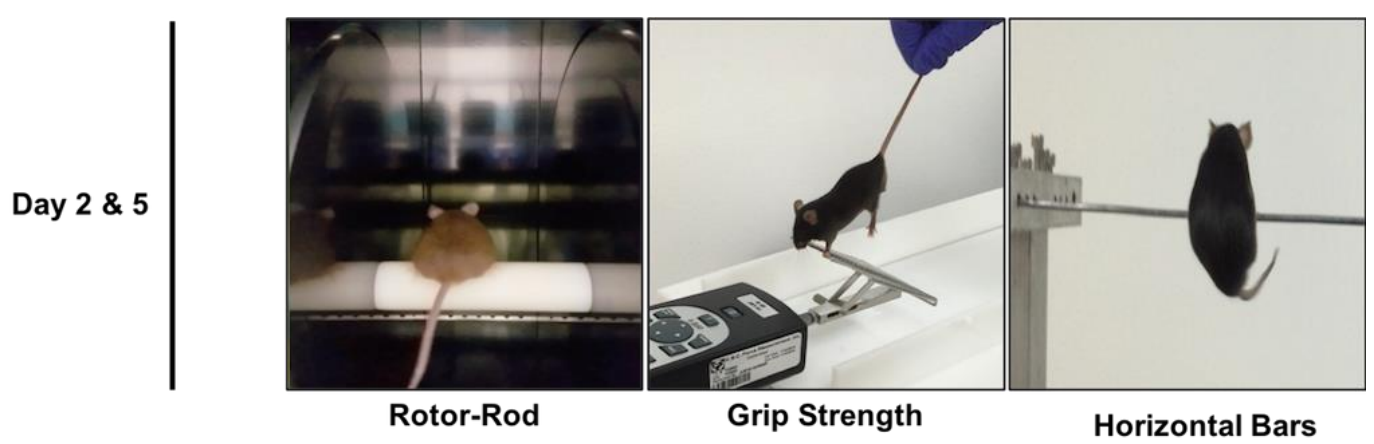

Figure 4.3 Representative images of motor skill behavioral assessments

On days 2 and 5 post-surgery, mice were assessed in 3 tests to assess motor performance and skill. $\mathrm{N}=4-8$ mice per treatment.

\section{Accelerating Rotarod}

The rotarod is an elevated rotating rod which exploits the natural fear of falling to evaluate balance, motor coordination and endurance. The Rotarod treadmill (San Diego Instruments) consists of a 1.25 in diameter cylindrical treadmill raised 18 in above the cushioned based connected to a computer-controlled motor driven drum which is programmed to operate in an accelerated mode. Each compartment possesses 7 photobeams placed strategically below the running rod. When the animal falls off the rotating rod, the amount of time elapsed from starting until the photobeam was passed 
was recorded by the computer automatically. All mice were naïve to the rotarod test until testing on day 2 post-surgery. Mice were placed on the elevated rod and the rod accelerated from 1 to $50 \mathrm{rpm}$ in $5.0 \mathrm{rpm}$ stages per 5 seconds with latency to fall recorded in seconds.

\section{Grip Strength}

The grip strength test (Chatillon force measurement; San Diego Instruments) assesses muscle weakness based on the instinctual tendency of mice to grasp objects with its forelimbs [391]. The apparatus is comprised of a push/pull digital force gauge meter connected to a computer for recording. Each mouse was weighed before forelimb grip strength test. The mice were held by the tail and lowered towards the computerized grid until it gripped the bar with both forepaws. The mouse was then gently and steadily pulled by the tail perpendicularly to the grid until the grid was released. Peak force was recorded in grams of force. Tests were repeated 5 times in 30 second intervals with the highest tension value being used as the animal's measure of strength. Peak grip was normalized to mouse body weight for each animal.

\section{Horizontal Bar}

Horizontal bars are an additional measure of forelimb strength and coordination using elevated 2- and 4-mm diameter bars (44 cm length) suspended by metal poles $(50 \mathrm{~cm}$ height). Holding the mouse by the tail, the mouse was placed on the benchtop and quickly slid backwards to align the mouse perpendicular to the bar and placed on the bar in the center of the two poles so that the mouse grasps the bar with its forelimbs only. Once grasping the bar, the mouse tail was released, and the timer started. Mice were tested on ability to hold on to each bar and/or traverse the bar to the end poles (supporting the bar). 
End point criterion was either falling from the bar, reaching the end pole, or reaching the maximum assessment time of 30 seconds. Duration of stay before falling or reaching the end pole was scored from $1-5$, with 5 being the highest score. Scoring criterion are listed in Table 4.1. Mice were tested on the $2 \mathrm{~mm}$ bar followed by the $4 \mathrm{~mm}$ bar (to increase difficulty) for 3 trials on each bar. The best scores for each bar were then added.

Table 4.1 Scoring used in horizontal bars assessment

$\begin{array}{cc}\text { Horizontal } & \text { Bars Scoring } \\ \text { Score } & \text { Time } \\ \mathbf{1} & 1-5 \mathrm{sec} \\ \mathbf{2} & 6-10 \mathrm{sec} \\ \mathbf{3} & 11-20 \mathrm{sec} \\ \mathbf{4} & 21-30 \\ \mathbf{5} & >30 \\ \mathbf{5} & \text { Pole }\end{array}$

\subsubsection{Tissue preparation and histology}

Following behavior experiments on day 5 , mice were sacrificed, and brains collected intact. Brains were fixed for 24 hours in $4 \%$ paraformaldehyde, then serially submerged in $15 \%$ and $30 \%$ sucrose for 24 hours, and finally embedded in in OCT compound and frozen for histology. Embedded frozen brain tissues were cut into coronal sections at $10 \mu \mathrm{m}$ thickness beginning at the forebrain and including the striatum (approximately ranging Section 18-25 in a mouse stereotaxic atlas [392]). Tissue sections were stained with cresyl violet acetate solution (Nissl) to stain the neuropil and identify the neuronal bodies within the brain as an indicator of decreased neuronal number. Briefly, sections were dehydrated in an ethanol series $(70 \%, 95 \%, 100 \%)$, incubated in cresyl violet for 20 minutes, rehydrated, and cleared with xylene. Slides were then mounted with Permount and cover 
slipped. Images of the striatum taken arbitrarily within a $600 \mu \mathrm{m} x / y$ distance from the injection epicenter were analyzed using a Zeiss (Germany) inverted fluorescence microscope with a 560 Axiovision camera in non-overlapping fields (Figure 4.4). In total, 2 animals per group, 2 tissues per animal, and 4 images per tissue were used for histological assessment with representative images shown at 100x. Cells that contained Nissl substance in the cytoplasm, loose chromatin and prominent nucleoli were considered to be neurons and were manually counted semi-blind, whereas in contrast, glial cells lack a conspicuous nucleolus and contain less endoplasmic reticulum and were excluded [393-395]. Niss ${ }^{+}$glia identified by these morphological criteria were not considered in the estimation of total neuron numbers. Quantification was performed at 40x magnification. The data were represented as the number of cells per $\mathrm{mm}^{2}$.
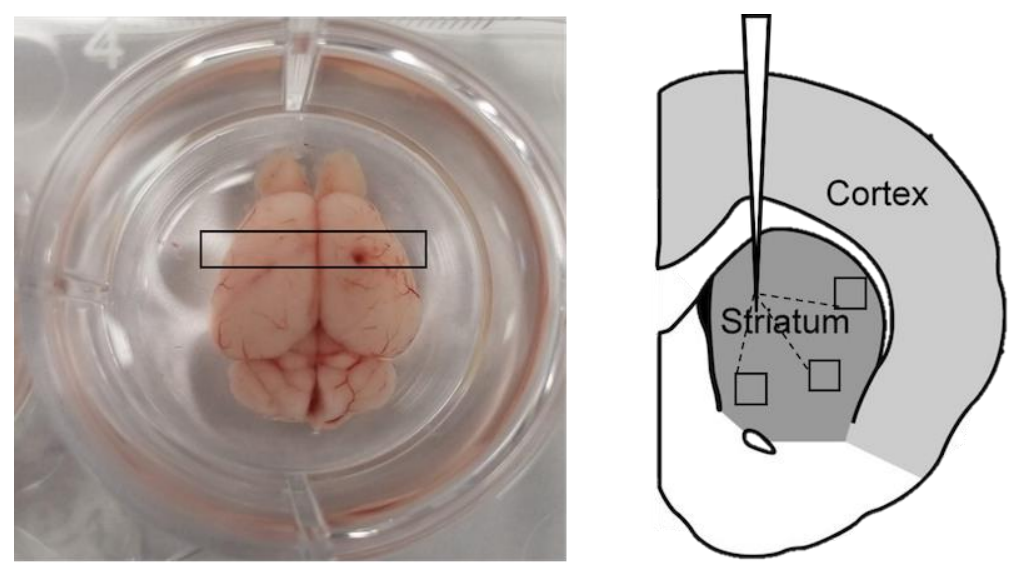

Figure 4.4 Post-fixed brain tissue and schematic representation of image sampling for histological assessment

On day 5 post-surgery, mice were sacrificed, brain tissues collected, fixed, and frozen. Tissues were sectioned by cryostat in the coronal plane at $10 \mu \mathrm{m}$ and every $6^{\text {th }}$ tissue used to Nissl staining. Images were taken approximately $600 \mu \mathrm{m}$ from the injection point. $\mathrm{N}=3$ mice per treatment, per strain. 


\subsubsection{Statistical Analysis}

All data were analyzed with Prism7 software. Results are reported as the mean of each treatment group \pm SEM based upon total number of mice assessed. Data were analyzed by either one-way or two-way analysis of variance (ANOVA), followed by post hoc analysis using Tukey's (treatment), Sidak's (strain) tests for multiple comparisons. P values of $<0.05$ were considered statistically significant.

\section{$\underline{4.3 \text { Results }}$}

With the introduction of cART, the manifestation of clinical symptoms in HIV patients has drastically decreased in severity with these deficiencies falling under the MND and ANI classifications of HAND [373]. In the study of the effects of Tat (and opiates) on brain function, many studies have focused on damage to the hippocampus and subsequent deterioration of cognitive function, memory, and behavior such as fear conditioning and anxiety [206, 207, 396]. Despite the occurrence of motor deficits being more prevalent in HAD within the pre-cART era, patients with HIV-associated neurological impairment may still show difficulties in motor coordination and fine motor skill, especially within the drug abusing population [397, 398]. Given the glial-induced inflammation and neuronal losses within striatal-derived cells observed in the previous chapters, we sought to determine if these effects could be recapitulated in vivo and translate to impaired motor skill, as well as determine what role Beclin 1 plays in regulating these behaviors. Following intrastriatal injection (Saline vs Tat) and subcutaneous pellet implantation (Placebo vs Morphine), mice were assessed for motor impairment. We first examined effects of Tat and morphine on mouse mortality (Figure 4.5). We found that while Tat/placebo treatment at $10 \mu \mathrm{g}$ to either C57BL/6J or Becn1 $1^{+/-}$mice showed no significant effects on mortality $(94 \%, 100 \%$ survival), Tat/morphine administration appeared to strongly affect survival with no 
difference in mortality between the strains $(50 \%, 50 \%$ survival). This may be a consequence of consistent morphine exposure since mice treated with saline/morphine also showed a decrease in survival and the C57BL/6J mice seemingly more affected by morphine than the Becn1+/- mice. This is particularly interesting since other studies have shown mice to tolerate $25 \mathrm{mg}$ and $75 \mathrm{mg}$ dosages of the extended release morphine pellets in studies [244, 399, 400]. Morphine is a well-studied analgesic with side effects that include constipation, respiratory depression, and hypothermia when entering the withdrawal state at approximately 24 hours after implantation which is when plasma drug concentrations are reported to peak [401-403]. As such, the study numbers shown represent the increased pool of test subjects to accommodate for these losses.

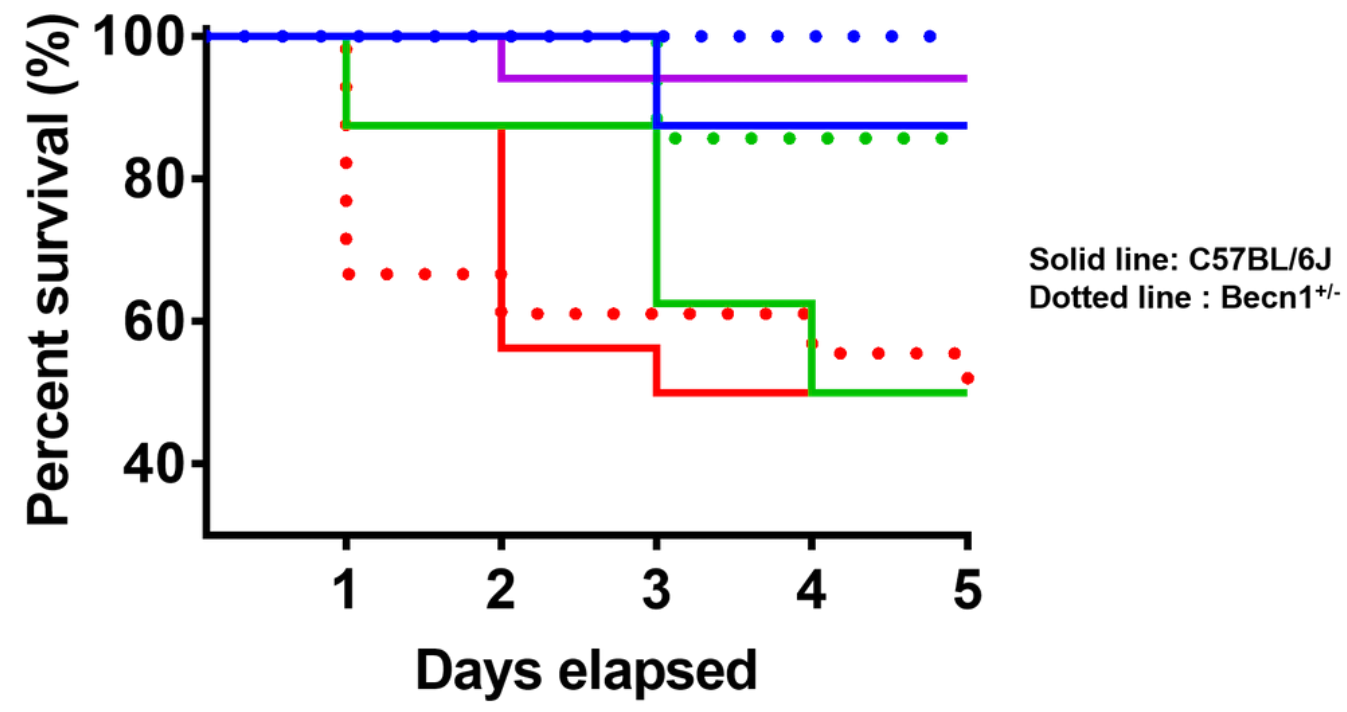

Saline + Placebo $\square$ Tat + Placebo $\square$ Saline + Morphine $\square$ Tat + Morphine

Figure 4.5 Mortality of mice following intracranial injection and subcutaneous pellet implantation

Morphine exposure led to significant mortality to both C57BL/6J and Becn1+/- mice over the 5-day experimental period with losses of up to $50 \%$ of mice. 


\subsubsection{Rotarod}

The accelerating rotarod test is a forced function test surveying utilizing fear of falling to assess motor activity as a function of gait, balance, coordination, and endurance [404, 405]. To examine the effects of Tat and morphine co-administration on motor skill and whether Beclin 1 deficiency is a beneficial or detrimental factor in mediating these outcomes, the accelerating rotarod assay was performed. To our surprise, we detected no significant differences in latency to fall on day 2 between saline/placebo and Tat/placebo treated mice for either strain of mice (Figure 4.6a). Instead, we were able to observe a morphine effect in both strains of mice, with a significant decrease in latency for both saline/morphine and Tat/morphine treated mice. This further indicates the lack of a Tat effect given the absence of any additive or synergistic effects with Tat and morphine co-exposure. Similar results were also seen at day 5 post-surgery with no apparent Tatinduced detriments in latency and a reduced morphine effect compared to day 2 (Figure

a.

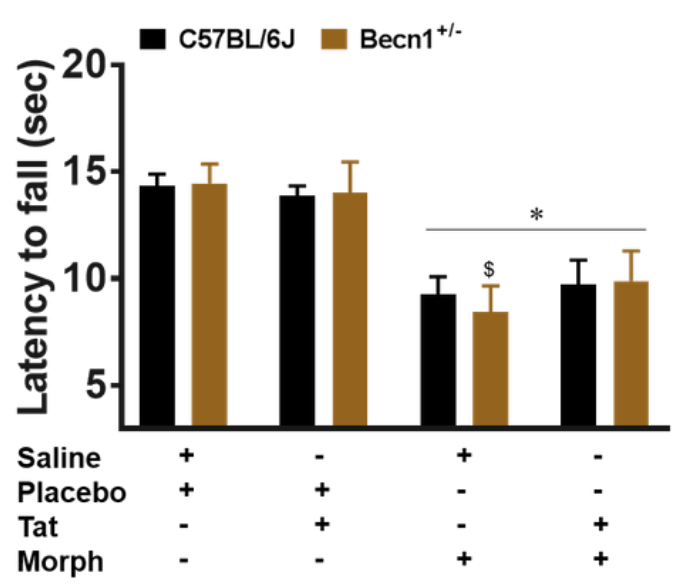

b.

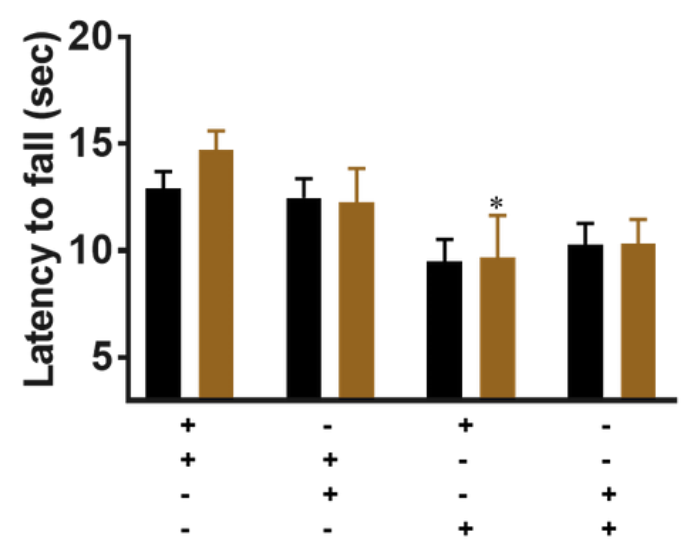

Figure 4.6 Tat exposure causes no detectable impairment of rotarod performance in C57BL/6J or Becn $1^{+/-}$mice

Four different groups (Saline/Placebo, Tat/Placebo, Saline/Morphine, Tat/Morphine) per strain were injected with $10 \mu \mathrm{g}$ of Tat protein into the striatum and implanted with a $25 \mathrm{mg}$ extended release pellet subcutaneously on day 0 . Motor activity was assessed on day 2 (a) and day 5 (b) post-surgery. There was no difference in Tat effect on day 2 or day 5. However, there was a morphine-induced decrease on both days. There was no difference between C57BL/6J or Becn1+/mice on either day. $\mathrm{P}<0.05$ * vs. Control; \# vs. C57BL/6J; \$ vs. Tat. Two-way ANOVA followed by Tukey's and Sidak's test. 
4.6b). Here we see no observable impairment of motor skills with Tat alone or in combination with morphine, rather, a morphine-induced diminishment which was indistinguishable between C57BL/6J and Becn1+/- strains.

\subsubsection{Forelimb Grip Strength}

The measurement of grip strength represents not only fine motor function but also neuromuscular capabilities which may be affected under experimental conditions [406]. As such, we sought to determine whether striatal-injected Tat, in the presence and absence of systemic morphine, would present as CNS motor deficits in C57BL/6J or Becn $1^{+-}$mice affecting the strength of the forelimbs. Similar to what was seen in the rotarod experiments, we detected no significant differences in peak grip with Tat/placebo exposure on day 2 post-surgery within or between the strains (Figure 4.7a). Interestingly, at 5 days post-surgery, there was a slight decrease in C57BL/6J peak grip strength when treated with Tat/placebo; however, this difference was not statistically significant from saline/placebo treated C57BL/6J mice or from Tat/placebo treated Becn $1^{+-}$mice. In addition, there was a significant Tat/morphine combined effect at 5 days post-surgery for both C57BL/6J and Becn1 $1^{+/}$mice which was significant from saline/placebo treatments (Figure 4.7b). These data suggest that there might be a time-dependent effect of Tat on neuromuscular strength in C57BL/6J mice but not Becn $1^{+/}$but that the addition of morphine causes significant impairment regardless of strain. 
a.

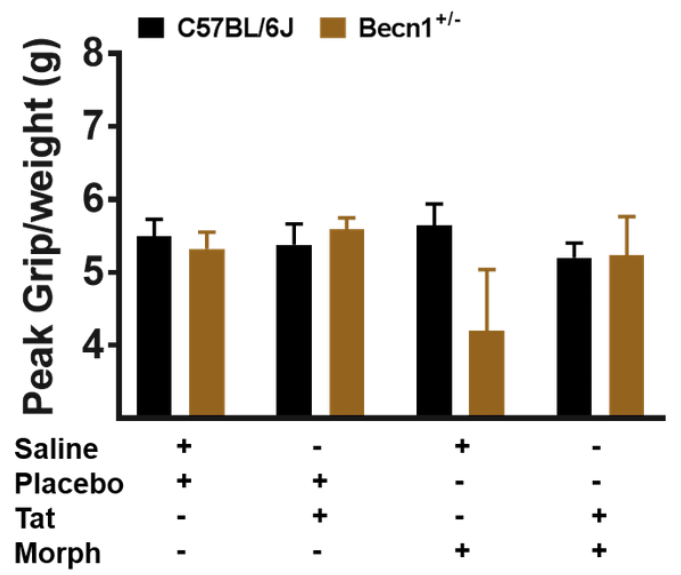

b.

Day 5

Figure 4.7 Tat and morphine exposure show delayed effects to grip strength

To further compare the effects of Tat and morphine exposure in vivo forelimb grip strength was tested within the 4 treatment groups of the C57BL/6J and Becn1 $1^{+/}$strains on day 2 (a) and day 5 (b). There was no detectable difference in grip strength with Tat exposure on day 2 and a moderate decrease on day 5 in C57BL/6J mice. Both C57BL/6J and Becn1+/- mice showed a significant difference in grip strength with co-administered Tat and morphine on day $5 . \mathrm{P}<0.05$ * vs. Control; \# vs. C57BL/6J; \$ vs. Tat. Two-way ANOVA followed by Tukey's and Sidak's test.

\subsubsection{Horizontal Bars}

We next examined how treated $\mathrm{C} 57 \mathrm{BL} / 6 \mathrm{~J}$ and Becn1+/- mice would fare at horizontal bars, an additional test which also requires forelimb strength and coordination for sufficient performance. At 2 days post-surgery, we were able to detect a Tat effect for both C57BL/6J and Becn1+- mice treated with Tat/placebo compared to the saline/placebo controls (Figure 4.8a). However, when examining the co-exposure effects with morphine, it appears as though the Tat effect is lost or potentially masked by the significant morphine effect. Comparing the saline/morphine and Tat/morphine treatments in C57BL/6J mice, there was a decrease, though statistically insignificant, in horizontal bar score and no difference with Becn $1^{+/}$. Only Tat/morphine treatment to Becn $1^{+/-}$mice showed a statistically significant difference from Tat/placebo treatment. At day 5 post-surgery the effect of Tat on Becn $1^{+-}$mice seemed to be reverted with no significant difference from saline/placebo treated mice (Figure 4.8b). Whereas in C57BL/6J mice, similar to what was seen at 2 days-post surgery, there was a small decrease in horizontal bar score, though 
this difference also not statistically significant. Interestingly, Tat/placebo treated Becn $1^{+/-}$ mice did out-perform similarly treated $\mathrm{C} 57 \mathrm{BL} / 6 \mathrm{~J}$ mice at both 2- and 5-days post-surgery which may suggest that lack of Beclin 1, at least in this particular motor assessment, reduces the effects of Tat on motor impairment. Once again, there was a significant morphine effect to both strains which may also have concealed the more understated Tat effects which were previously detected; however, this morphine effect was particularly apparent with the Becn $1^{+/-}$mice, with Tat/morphine being significantly different from either the saline/placebo or the Tat/placebo. From this, we can infer that a test which assess coordinated motor skill and muscle strength like the horizontal bars test can be subtly affected by Tat intrastriatal exposure in a time-dependent fashion but is drowned out with morphine co-administration, particularly in Becn1 ${ }^{+/-}$mice.

a.

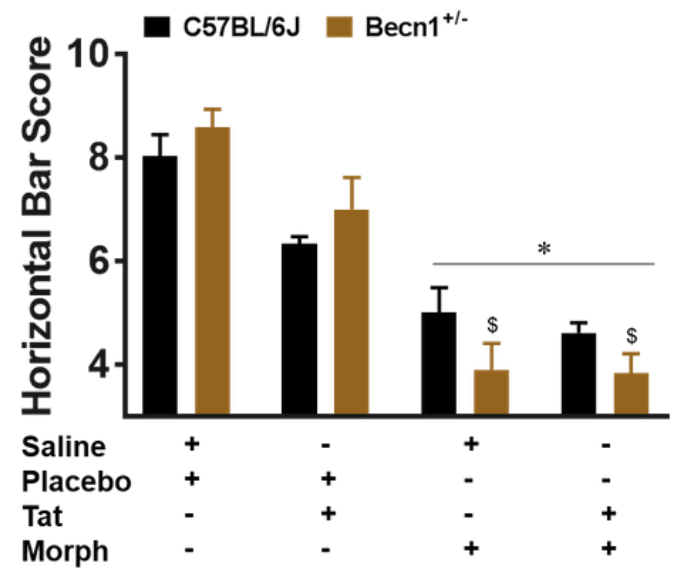

b.

Day 5

Figure 4.8 Becn1+/- mice outperform C57BL/6J exposed to Tat but not co-treated with morphine in horizontal bars

Horizontal bars assessment of motor coordination was done with the 4 treatment groups of the C57BL/6J and Becn 1+/- strains on day 2 (a) and day 5 (b). There was a decrease in horizontal bars score with Tat treatment on day 2 in both strains though there was no significant difference between strains. Morphine also has a marked effect on day 2. Only C57BL/6J mice were still affected by Tat on day 5 and morphine effects were lessened but still present with Tat and morphine co-exposure causing minor decreases. $\mathrm{P}<0.05$ * vs. Control; \# vs. C57BL/6J; \$ vs. Tat. Two-way ANOVA followed by Tukey's and Sidak's test. 


\subsubsection{Striatal neuron losses caused by Tat and morphine co-exposure}

The detrimental effects of viral proteins on neuronal populations in vivo have been reported for brain areas including the striatum, hippocampus, and substantia nigra [241, 407-409]. Following motor skill assessment, histological sections were stained by Nissl method to identify neuronal morphology and approximate any changes in neuronal number within the striatal area of interest (Figure 4.9a). Cells were identified as neurons based upon guidelines described by Garcia-Cabezas et al [393]. Nissl staining of the striatum revealed a significant reduction in the number of neurons of both $\mathrm{C} 57 \mathrm{BL} / 6 \mathrm{~J}$ and Becn1+/- mice (1.4-fold, 1.5-fold respectively) which had been exposed to Tat (Figure $4.9 \mathrm{~b}, \mathrm{c})$. Additionally, we also observed an enhanced toxicity in C57BL/6J neurons within the Tat/morphine treatment group (1.8-fold decrease from control, 1.3-fold decrease from Tat/placebo) which was unseen in the similarly treated Becn $1^{+/-}$group (1.1-fold decrease from control.) To our surprise, Tat/morphine treated mice displayed less neuronal death than the Tat/placebo treatment group (1.4 -fold increase from Tat/placebo). Saline/morphine treatment exhibited no neuronal losses to either C57BL/6J or Becn1+/mice and may indicate mild neuroprotection $[410,411]$. Our results show that Becn $1^{+/-}$ mice present less damage to neurons upon intrastriatal Tat and systemic morphine exposure compared to $\mathrm{C} 57 \mathrm{BL} / 6 \mathrm{~J}$ mice which provides an additional glimpse into the role of Beclin 1 in mediating neuropathology. 
a.

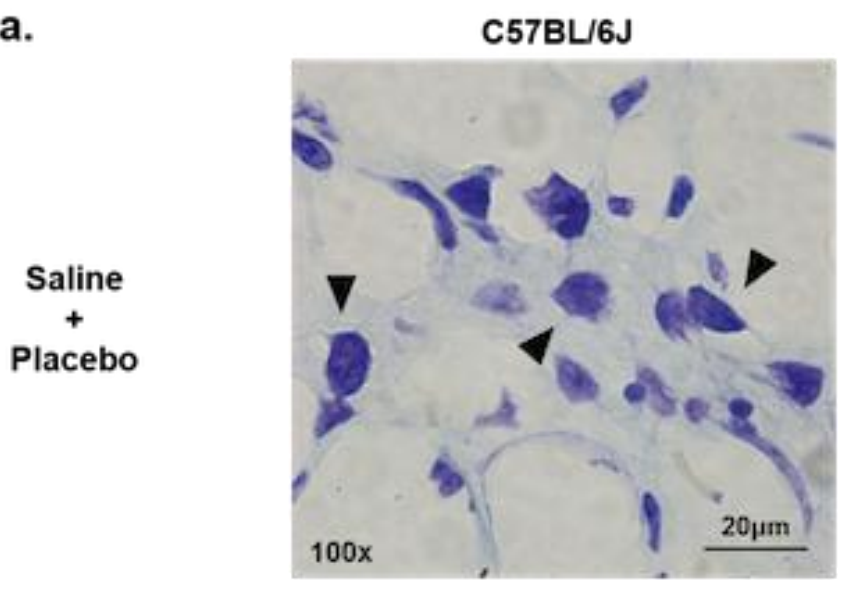

Tat

Placebo

Saline

$+$

Morphine

Tat

Morphine
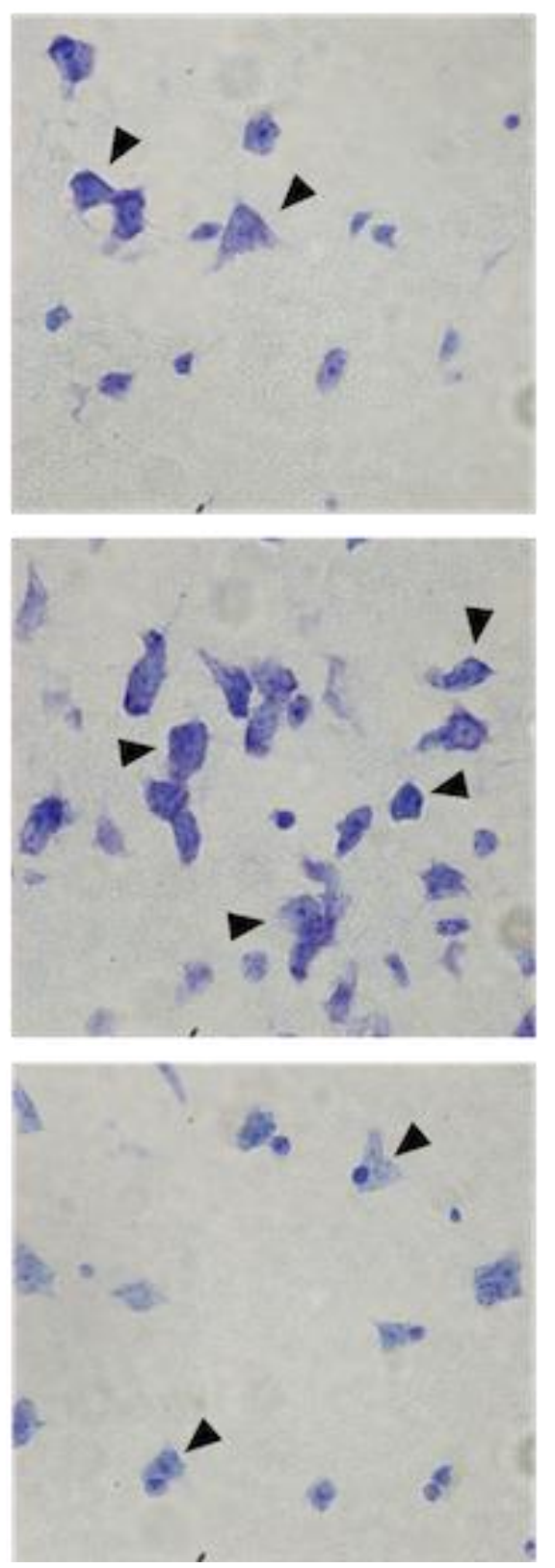
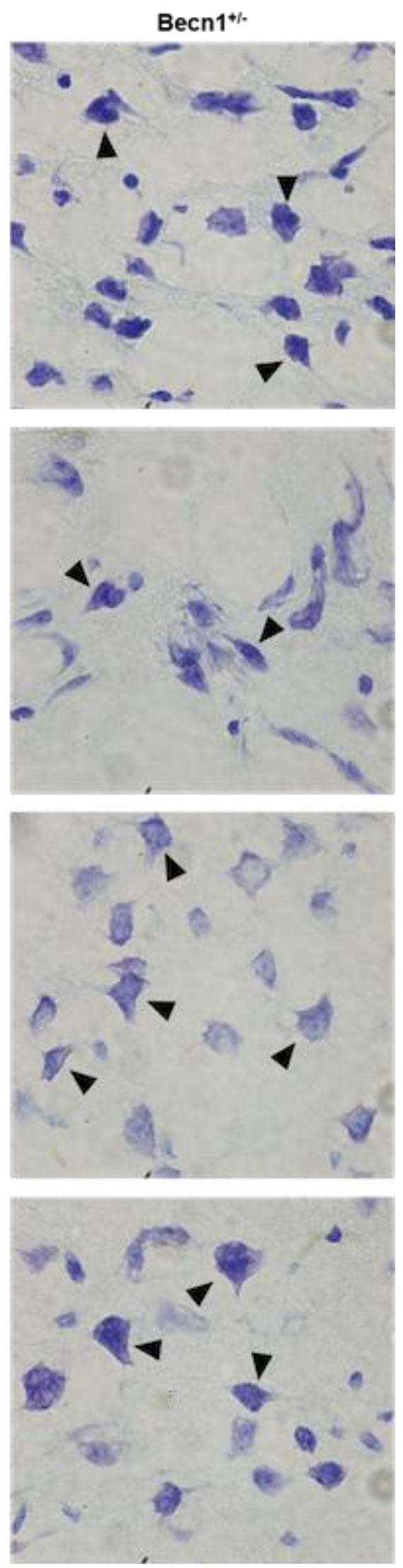
C57BL/6J

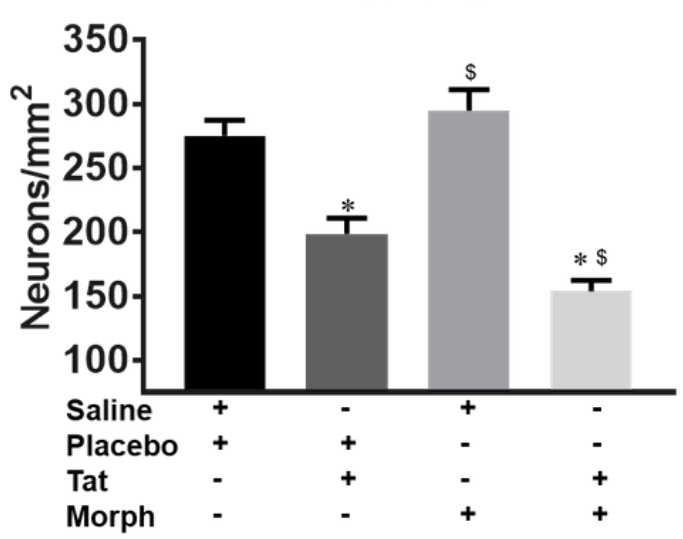

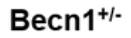

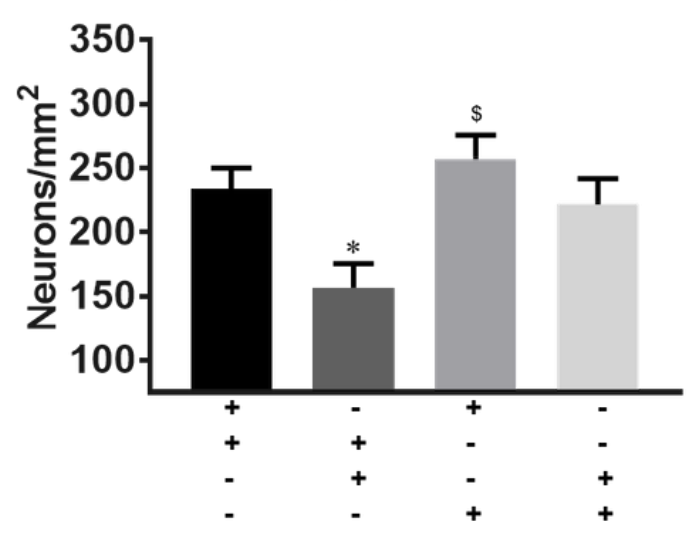

Figure 4.9 Tat and morphine co-exposure causes less neuronal loss in the Becn $1^{\text {+/- }}$ striatum Staining of striatal tissue for Nissl substance in saline/placebo, Tat/placebo, saline/morphine, Tat/morphine treated C57BL/6J and Becn1 ${ }^{+/-}$mice (a). Panels show sections at $100 x$ magnification of the striatum in coronal sections (a). Scale bars: $20 \mu \mathrm{m}$. Black arrows indicate neurons. Neurons were quantified for each treatment and averaged based upon the striatal areas captured $(\sim 0.3$ $\mathrm{mm}^{2}$ ). Cell counting showed a reduced number of Nissl-stained striatal neurons when treated with Tat in both C57BL/6J and Becn1+- mice. Morphine co-administration enhanced neuronal loss in C57BL/6J mice but not Becn1+- mice. $\mathrm{N}=2$ mice per group, 2 tissues per mouse, 4 images per tissue. $\mathrm{P}<0.05$ * vs. Control; \$ vs. Tat. One-way ANOVA followed by Tukey's test.

\section{$\underline{4.4 \text { Discussion }}$}

In this chapter, we sought to examine the role of Beclin 1 in mediating Tat and morphineinduced motor impairment in vivo. As in the previous chapters, to do this we make use mice possessing a monoallelic deletion of the Becn1 gene but here we use 3-5-month-old mice which underwent intra-striatal Tat exposure and subcutaneous morphine pellet implantation. In the previous chapter, we observed the death of striatal neurons in vitro following exposure to Tat and morphine. In addition, this death was significantly enhanced by the inclusion of conditioned media from C57BL/6J glia treated with Tat and morphine but less so when treated with conditioned media from Becn1+/- glia. From this, we questioned whether the observed striatal neuron death could be translated to in vivo motor impairment. The basal ganglia are a group of sub-cortical structures within the brain comprised of the dorsal striatum (caudate and putamen), ventral striatum (nucleus accumbens and olfactory tubercle), globus pallidus, substantia nigra, and subthalamic 
nucleus. These areas are primarily responsible for motor control in addition to motor learning, executive functions, and behavior [412]. Early studies of AIDS dementia reported clinical features similar to those seen in PD, a neurodegenerative disease of attributed to dopaminergic neuron death in the substantia nigra. These included bradykinesia, impaired manual dexterity, postural instability, rigidity, and gait abnormality [41, 42]. Neuropathological studies both in humans and animals have shown that the striatum is an area with heavy HIV burden and so the presentation of numerous motor deficits in early patients was quite prevalent, even without accompanying cognitive dysfunction $[378,385]$. Fast forward several decades, and cART has radically changed the landscape of living with HIV with a severely decreased incidence of HAD; however, neurocognitive impairments still remained high $[373,413]$. Whether due to lack of cART penetrance, inherent CART toxicity, or continued presence of HIV proteins in the CNS, there is still significant need to understand the driving forces leading to HAND and potential therapies to negate them, especially in the drug abusing population where neurodegeneration is not only enhanced but occurs at a faster rate.

In these studies, we injected C57BL/6J and Becn $1^{+/-}$mice with either saline or $10 \mu \mathrm{g}$ of Tat and subcutaneously implanted either a placebo pellet or $25 \mathrm{mg}$ extended-release morphine pellet (Figure 4.1) and assessed for deficits in motor skill 2 and 5 days after surgery. We saw no observable effect of Tat on rotarod performance for either strain of mice, but a significant effect of morphine (Figure 4.6). Studies which have looked at the effect of HIV or its viral proteins on rotarod performance have been quite varied in their results with some studies finding significant impairment in accelerated rotarod performance [409, 414-417], and some studies finding no differences [214, 396, 399, 418420], as we have here. There were, however, two significant differences between those 
studies and the ones performed here. The first difference lies is in how the mice were exposed to Tat. Many of the studies found in the literature make use of transgenic mouse models such as the doxycycline-inducible Tat transgenic mouse or the HIV transgenic rat models in their rotarod assessments of motor impairment as briefly summarized in Chapter 1.11.1. Those established transgenic mouse models used to study HIV neuropathogenesis are designed to produce virus/viral proteins under the control of a GFAP promoter so that the protein(s) of interest are produced within the brain upon the introduction of doxycycline into the diet [227]. As such, it is difficult to know exactly how much of a protein such as Tat is being produced in a specific area such as the striatum. Conversely, our study injects a pre-determined concentration of Tat directly into the striatum. Furthermore, our study presents a one-time exposure to Tat whereas the Tat transgenic models display a continuous production of the protein. Given that Tat is readily oxidized or taken up in to cells and has a short half-life [7, 421], injection of Tat may provide only brief snapshot of direct effects whereas the Tat transgenic mice are exposed to Tat more chronically which may explain why we did not see the pronounced difference in rotarod performance. In addition, other studies have used up to $25 \mu \mathrm{g}$ of the viral protein Tat when investigating its effects on neurodegeneration so it is possible that a higher concentration may be necessary to portray in vivo behavior impairments. While Tat concentrations in the serum of HIV patients have been reported to be between 2 and 40 $\mathrm{ng} / \mathrm{mL}$, it is often through that concentrations in the brain are higher, and as such, future experiments at a higher concentration would still be clinically relevant [342, 422, 423]. The second difference lies in the assessment of rotarod performance in that the present study did not train the mice prior to surgery for assessment. It was also curious to see the distinct effect on rotarod performance caused by morphine in both strains. Similar to the literature on rotarod performance in HIV models, results on mouse/rat motor coordination following 
morphine treatment are also divided with some reporting no differences with morphine exposure [424, 425], and others stating that morphine can compromise motor function at antinociceptive doses [426-429]. One study by Fitting et al which used the inducible Tat transgenic mouse model reported that poor rotarod performance was tied to rapid increases in Tat within the striatum and that acute morphine administration could increase the latencies of mice on the rotarod [209]. Taken together, more studies at higher concentrations of Tat may be needed to gain a better understanding of whether Beclin 1 plays a translational role in motor coordination.

When we surveyed the effects of Tat and morphine on forelimb grip strength, we also saw mild effects, many of which were not statistically significant and were apparent only on day 5 after surgery (Figure 4.7b). Neurodegenerative diseases where the basal ganglia is affected will often show reduced strength of forelimbs, although we did not detect any in these studies [430]. It should be noted that although we normalize for body weight, other factors such as motivation to continue gripping the bar have been shown to affect the assessment of rodent grip strength [431]. One study examining the gender influences on Tat-induced neurological impairment using the inducible Tat transgenic mice however reported that forelimb strength was significantly reduced in male $\mathrm{Tat}^{+}$mice compared to female Tat $^{+}$or Tat mice of either gender [417]. Similar to what was seen in the rotarod, it is possible that at a higher exposure of Tat, deficits may become more apparent. Interestingly, there seemed to be an interactive effect, though independent of strain, in Tat and morphine treated mice on day 5 . This has also been shown in studies on the effects of morphine on behavioral task performance in SIV-infected rhesus macaques [432]. Animals showed reduced performance in the behavioral tasks assessed like forelimb force, which was significantly further reduced after infection. Although this study uses large 
animals in a chronic morphine paradigm, it demonstrated an effect of morphine to produce behavioral deficits and act synergistically with SIV to exacerbate motor deficits.

Once termed the "string test" or "'coat hanger test", the horizontal bars assessment measures both forelimb strength and coordination [433]. A combination of both the motor programming of the basal ganglia and the posture programming of the cerebellum, the horizontal bars test has been used to study motor defects caused by targeted null mutations and surgical/neurochemical lesions within those areas of motor ability [434]. Given that the mouse's ability to grip the bar is inversely proportional the diameter of the bar, studies use increasing sizes $(2 \mathrm{~mm}, 4 \mathrm{~mm}, 6 \mathrm{~mm})$ to increase difficulty and better differentiate mouse ability [433]. In these studies, we observed a Tat effect in both C57BL/6J and Becn1 ${ }^{+/-}$mice on day 2, but only the C57BL/6J mice retained the deficits on day 5. Here we also detected a significant morphine effect to both strains of mice on both days of testing which was particularly prominent in Becn $1^{+/}$mice (Figure 4.8). From these results we may speculate that the lack of Beclin 1 might trigger a delayed compensatory or recovery mechanism which rescues the Tat effects on behavior by day 5; however, in terms of Tat and morphine co-exposure, the addition of morphine may mask any benefits of Beclin 1 deficiency or simply does not have synergistic effects with Tat. While the manifestations of motor impairment in Tat and morphine co-exposed C57BL/6J and Becn $1^{+/}$mice might have been mild at the given concentrations of viral protein, deficits appear to be time dependent.

Many of the deficits in neurodegenerative diseases stem from neuronal losses within the brain locations responsible for their function. The neuropathology and clinical presentation including anxiety, memory, learning, and motor deficits correlated with HAND are 
associated with degeneration of sub-cortical structures such as the amygdala, hippocampus, and basal ganglia $[13,435]$. Despite observing only modest impairment of motor function in certain assessments with Tat exposure alone or in combination with morphine within the strains, we were able to find significant differences in estimated neuronal number both with respect to treatment and strain (Figure 4.9). It is necessary to note this crude quantification did not span the entire length of the striatum and it is possible the areas assessed were not areas of significant impairment. These neuropathological analyses are consistent with our studies here and others presented elsewhere of neuronal Tat toxicity [199, 241, 408, 409]. Similar to the existing literature on Tat effects in locomotor skill, studies on the histological effects of Tat exposure are at odds with some reports finding no significant effect on the number of striatal neurons [436], and others finding great neuronal tissue losses [241, 408]. Opposite with our findings here, one such study stated that Tat production in the rat striatum caused impairment of behavioral performance, but found no extensive neuronal loss, attributing deficits to neuronal and synaptic dysfunction [409]. Likely, it is a combination of both striatal neuron death and dopaminergic malfunction leading poor motor performance. For example, Tat has been demonstrated to diminish expression of tyrosine hydroxylase $(\mathrm{TH})$, the rate determinant of dopamine synthesis, in the substantia nigra following striatal injection, in addition to the loss of $\mathrm{TH}^{+}$neurons, and development of subclinical PD-like disease when treated with the stimulant amphetamine [437]. Although the studies here lack the quantitative backing of stereology, it is an initial step into understanding how targeting of Beclin 1 in the context of Tat and opiate toxicity may be neuroprotective. As the study of Beclin 1 and autophagy in the context of Tat and opiate co-exposure is expanded, future studies will also look at other afflictions and diseases caused by HIV exposure. 
In conclusion, the aforementioned data provide further evidence that exposure to Tat plays a meaningful role in the deterioration of motor function experienced by HAND patients and worsened in the drug abusing population. The autophagy protein Beclin 1 may yet still play a role in the manifestation of Tat and morphine-induced deficits. The absence of discernable motor impairments between either strains or with Tat administration in certain assessments, coupled with the presentation of neuronal loss within the striatum make for a complicated landscape requiring additional study. One possibility could be that the induced damage might not yet be sufficient to destabilize the functionality of striatal motor circuits. In addition, motor function is a coordinated effort with other brain areas such as the cerebellum which will also play a role in motor performance. Given the data presented in previous chapters, future studies can advance the work further by incorporating more advanced models of HIV CNS infection to establish Beclin 1 as a glial target for reducing HIV and opiate induced neurodegeneration capable of rescuing motor and potentially cognitive deficits. 


\section{CHAPTER 5: FINAL CONCLUSIONS AND FUTURE DIRECTIONS}

The goal of this dissertation was to investigate the mechanisms underlying HIV and opiateinduced exacerbation of neurodegeneration. Here we provide literature and experimental rationale supporting the role of the autophagy pathway, specifically the initiation protein Beclin 1, in mediating viral inflammation, neurotoxicity, and motor impairment. To do this, we utilize a heterozygous Beclin 1 deletion mutant, Becn $1^{+/}$, which displays an approximate $60 \%$ reduction in Beclin 1 expression. Given that mice are not permissive to HIV infection without genetic modification, we instead use the viral protein Tat which is secreted by HIV infected cells and demonstrates significant neurotoxicity [241, 438]. This is also currently relevant in terms of cART adherent patients which may exhibit reduced or undetectable levels of HIV in circulation but may still be producing Tat in locations of viral reservoirs such as the CNS.

We first sought to characterize what effects a reduction in Beclin 1 expression would have on glial cell responses to Tat and opiate exposure. Tat is an established inducer of cytokine and chemokine secretion from microglia and astrocytes, inflicting widespread neuroinflammation as well as dysregulating typical functions performed by these cells within the microenvironment [66, 100, 106, 439]. Reduced expression of Beclin 1 in mixed glial cultures significantly reduced Tat and morphine-induced secretion of inflammatory molecules like RANTES, MCP-1, and to a smaller extent IL-6 as well as intracellular $\mathrm{Ca}^{2+}$. From this it can be taken that Beclin 1 can partake in the regulation of neuroinflammation. However, Becn $1^{+/}$glia did not appear to rescue oxidative stress in terms of ROS and NO production. This may be a pitfall of the study design and execution based upon the readings obtained for the positive control $\mathrm{H}_{2} \mathrm{O}_{2}$. In general, $\mathrm{H}_{2} \mathrm{O}_{2}$ release should increase 
over time; however, here we observed an elevated but fairly steady state level of DCF fluorescence. From this it can be hypothesized that the signal reached maximally detectable levels and corresponding readings may be lacking the necessary sensitivity. Further optimization of the $\mathrm{CM}-\mathrm{H}_{2}$ DCFDA dye concentrations are necessary in conjunction with validation by fluorescent microscopy. Additionally, we examined was the effects of Tat and morphine on the glial autophagy pathway which is a key pathway abused by HIV. We found that where Tat and morphine increased expression of autophagy proteins like Beclin 1, p62/SQSTM1 and LAMP1 in wild type C57BL/6J glia, this effect is negated in Becn1+/- without significant glial toxicity. These effects on the autophagy pathway suggest that Tat and morphine trigger the autophagy pathway (indicated by elevated Beclin 1 expression) but that maturation and degradation are inhibited (indicated by elevated p62/SQSTM1 and LAMP1), potentially causing autophagosome accumulation. By reducing Beclin 1 expression in Becn $1^{+/}$, it is possible to diminish, but not completely abrogate, autophagy activation caused by Tat and morphine exposure. Despite these findings, the prevention of autophagosome accumulation through reducing Beclin 1 can only be confirmed by electron microscopy. In addition, confirmation of impaired autophagic flux should be confirmed with the use of RFP-GFP-LC3B tandem sensors which use fluorescent tags to indicate autophagosome and lysosome colocalization [308].

From the basis of this chapter, future work can look at the exact mechanisms causing Tat and morphine to trigger autophagy. One can speculate it involves ion dysregulation and stress sensing mechanisms such as JNK or MAP kinase pathways. We and others have shown that Tat and morphine is able to increase intracellular calcium which is prevented in Becn $1^{+-}$glia; this ionic imbalance may subsequently lead to aberrant signaling and 
activation of stress responses. MAPK signaling serves many roles in cell survival with JNK and p38 MAPK being shown to be activated by pro-inflammatory cytokines and environmental stress [440]. In addition, HIV or viral protein exposure can activate JNK and/or p38 MAPKs with inhibition of these pathways reducing toxic effects [60, 441]. Provided that JNK has the capability of phosphorylating BCL-2, separating it from Beclin1, it is possible that such pathways may explain the Tat-mediated activation of the autophagy pathway [442]. Currently, ongoing studies are interested in the protein-protein interactions going on within glial cells treated with Tat. Since Tat easily crosses cell membranes and its basic domain can interact with many structures, it is curious what, if any, interacting partners it has in the autophagy pathway. The viral protein Nef is known to interact with Beclin 1 [16], and we have also shown a significant Nef effect in respect to inflammatory molecule secretion (Figure 2.6). Since we have shown that reduction of Beclin 1 has inflammation and autophagic dysregulation alleviating capabilities within glia, determining direct interacting partners would further the understanding of these mechanisms.

Next, given that the HIV and opiate effects on glia converge on neurons, we ventured to determine how Beclin 1 reduction would affect neuron response to these stressors. We found that Becn1 ${ }^{+/-}$neurons are highly sensitive to injury and stressors such as Tat and morphine. While co-exposure to Tat and morphine caused elevations in intracellular calcium accumulation and dendritic beading in C57BL/6J neurons as has been previously published [204, 316], these were enhanced in Becn1 ${ }^{+/}$neurons, although Tat alone had a higher effect than Tat and morphine combined (Figures 3.2 and 3.3). This effect was further showcased upon assessment of neuronal viability by time-lapse recording showing significant neuronal death with or without treatment (Figure 3.4). Given that HIV neuropathogenesis is a glially-driven and with the promising reductions in inflammation 
seen in Becn $1^{+/}$glia, we desired to see how neuronal outcome might vary with exposure to the indirect toxicity of supernatant from Tat and morphine treated C57BL/6J and Becn1/glia. We found that C57BL/6J neurons treated with Becn $1^{+/}$supernatant had more favorable outcomes than those treated with C57BL/6J supernatant (Figure 3.5). When postulating what soluble components within the supernatant could be mediating these effects, we found that in addition to the stemming of inflammatory molecule secretion, this may also be due to increase secretion of neuroprotective growth factors (Figure 3.6).

Our studies on the neuro-glial interactions are only in the preliminary stages. Now that we have found that reduced Beclin 1 in glia can reduce the Tat and morphine toxicity to neurons, future studies will examine neuronal functionality which can be just as important as neuronal viability. These would include studies on neurotransmitter release which is reported to be dysregulated with Tat exposure [416]. For example, the striatum is a location with predominantly GABAergic medium spiny neurons but receives both dopaminergic and glutamatergic inputs from various sources [412]. As such, glutamate uptake and dopamine release from these neurons are key aspects of function. One study on the effect of Tat and methamphetamine in rat striatal neurons showed that the treatment lead to striatal dopamine deficit which contributed to the manifestation of motor impairment [416]. The evaluation of neuronal viability performed here also requires further exploration into the mechanisms of neuronal death whether apoptosis dependent or independent pathways. Future studies can also take the co-culture paradigm further by direct contact of glia and neurons. Assessing how neuronal survival and responses (including neuronal autophagy) to Tat and morphine shift in the presence of Becn $1^{+/}$glia can provide confirmation that targeting of glial Beclin 1 is a viable therapeutic strategy for HIV and opiate neuropathogenesis. 
Finally, we wanted to see if the beneficial results seen in Becn1 $1^{+-}$glia and with Becn $1^{+/-}$ supernatant on neurons could be translated into positive outcomes in motor impairment in vivo following Tat and morphine exposure. To do this we performed stereotaxic surgery on $\mathrm{C} 57 \mathrm{BL} / 6 \mathrm{~J}$ and Becn $1^{+/-}$mice and injected $10 \mu \mathrm{g}$ of Tat into the mouse striatum while also implanting a $25 \mathrm{mg}$ extended-release morphine pellet (Figure 4.1). These surgical methods have been done before for analysis of glial dysfunction and tissue damage, but never in the context of autophagy [242, 244]. At 2- and 5-days post-surgery, motor activity was assessed by three motor coordination tests: rotarod, forelimb grip strength, and horizontal bars. To our surprise, we were unable to detect any Tat effects in rotarod on either day for either strain but noted a significant morphine effect to both strains on day 2 (Figure 4.6). Forelimb grip strength performance showed a similar lack of effect on day 2; however, on day 5, we observed a Tat effect in C57BL/6J mice but not Becn1 ${ }^{+/}$mice, although not statistically significant (Figure 4.7). Both C57BL/6J and Becn1+/- mice showed an apparent Tat and morphine co-exposure effect. There were somewhat different results in the horizontal bars assessment of motor coordination (Figure 4.8). On day 2 we perceived a Tat effect in both C57BL/6J and Becn $1^{+/-}$mice; however, the observed morphine effect was so robust, we theorize that it may be obscuring any potential synergistic effects of Tat and morphine. Interestingly, on day $5 \mathrm{C} 57 \mathrm{BL} / 6 \mathrm{~J}$ mice retained the observed Tat effect whereas Becn1+/- mice returned to control levels; the morphine effect however was still potent, though not to the extent of day 2 performance. Due to the varied results which can be quite common in animal studies, we found that the behavior data may not correlate with the in vitro results observed in Chapters 2 and 3 . We also inspected histological changes within the striatum of treated mice by Nissl staining and found significantly reduced neuronal numbers in C57BL/6J mice treated with Tat that was 
exacerbated by morphine co-exposure, whereas Becn $1^{+/-}$mice showed losses only with Tat treatment. It is difficult to reconcile the seemingly contradicting results within the motor assessments and tissue evaluation. One can postulate that the neuronal losses seen within the tissue may not yet be extensive enough to translate into motor impairment. These studies were done for up to 5 days whereas in HIV patients, Tat exposure is more continuous allowing for more extended attack on the CNS. Tat protein is also rapidly oxidized so it may be necessary to have more continuous exposure to the protein for detectable outcomes. However, it must be noted that assessment of Nissl staining was done manually instead of the more appropriate and accurate stereological quantification. This would also require screening of the entire striatum rather than the anterior most sections due to variable distribution and projections of neurons throughout the striatum. Another consideration would be the systems at play in motor coordination. Notably, each of the motor assessments yielded somewhat different results, likely because each test appraises slightly different aspects of motor coordination: rotarod measures motor coordination, grip strength measures muscular ability, and horizontal bars measure a mix of the previous two [434].

There are many options for future studies to advance the work presented here. Continuing with this model, it might be necessary to increase the amount of Tat used for injections or to concentrate the protein to reduce the injection volume. While we detected no backflow of Tat from the injection site, such a volume $(10 \mu \mathrm{L})$ may leak into the ventricles or drain into the CSF or lymphatic system. One study performed $1 \mu \mathrm{L}$ canula injections of Tat at the same concentration used in our studies $(10 \mu \mathrm{g})$ in the rat striatum and saw significant motor impairment and neuronal death [416]. Separately, additional assessments and brain locations could be incorporated into the study design. For example, other brain locations 
like the hippocampus are also affected by heavy HIV burden [207, 378, 379, 408]; it would be pertinent to see how other brain structures are affected by Tat and morphine coexposure within the Becn $1^{+-}$mice. Nociception is another clinically relevant avenue for study with these mice. Given that neuropathic pain is an issue afflicting HIV patients, how HIV and opioids interact to modulate pain perception is of particular importance, especially in the current opioid epidemic climate [209, 443]. Alternatively, the work could proceed with another, more advanced, injection model using the mouse permissive EcoHIV virus. As reviewed earlier (Chapter 1.11.3), this modified virus is infectious in mice and though it does not cross into the brain efficiently, can be directly injected into the brain and replicated vigorously [238]. Infecting Becn $1^{+/}$mice with this chimera virus (in combination with morphine pellet implantation) would provide a greater understanding and further proof of concept of the therapeutic potential of targeting Beclin 1 in HIV. As studies move more into treatment and translational paradigms to be used as adjuvants in conjunction with CART, humanized mouse models of HIV will be useful to see the therapeutic relevance of reducing Beclin 1 in the aim of ameliorating the neurodegenerative effects of HIV both in the aging AIDS population and the drug abusing population. Based on our results here, it would be essential to target glia specifically, as to not hinder neuronal survival by reducing autophagy in those cells as seen in Figure 3.4. Currently our lab has explored various drug delivery systems for delivering siRNA against Beclin 1 to the brain [257, 258], and with the studies shown here, we can continue to fine tune therapeutic strategies for what are hopefully widespread benefits to the HIV population and beyond.

\section{REFERENCES}

1. Weiss, R.A., How does HIV cause AIDS? Science, 1993. 260(5112): p. 1273-9.

2. UNIAIDS. Global AIDS Update 2017. 2017 [cited 2018 September 18]; Available from: http://www.unaids.org/en/resources/fact-sheet. 
3. Alexaki, A., Y. Liu, and B. Wigdahl, Cellular reservoirs of HIV-1 and their role in viral persistence. Curr HIV Res, 2008. 6(5): p. 388-400.

4. Shaw, G.M. and E. Hunter, HIV transmission. Cold Spring Harb Perspect Med, 2012. 2(11).

5. Dinkins, C., J. Arko-Mensah, and V. Deretic, Autophagy and HIV. Semin Cell Dev Biol, 2010. 21(7): p. 712-8.

6. Gallo, R.C. and L. Montagnier, The discovery of HIV as the cause of AIDS. N Engl J Med, 2003. 349(24): p. 2283-5.

7. Nath, A., Human immunodeficiency virus (HIV) proteins in neuropathogenesis of HIV dementia. J Infect Dis, 2002. 186 Suppl 2: p. S193-8.

8. Ivey, N.S., A.G. MacLean, and A.A. Lackner, Acquired immunodeficiency syndrome and the blood-brain barrier. J Neurovirol, 2009. 15(2): p. 111-22.

9. Davis, L.E., et al., Early viral brain invasion in iatrogenic human immunodeficiency virus infection. Neurology, 1992. 42(9): p. 1736-9.

10. Williams, D.W., et al., Mechanisms of HIV entry into the CNS: increased sensitivity of HIV infected CD14+CD16+ monocytes to CCL2 and key roles of CCR2, JAM$A$, and ALCAM in diapedesis. PLoS One, 2013. 8(7): p. e69270.

11. Eugenin, E.A., et al., CCL2/monocyte chemoattractant protein-1 mediates enhanced transmigration of human immunodeficiency virus (HIV)-infected leukocytes across the blood-brain barrier: a potential mechanism of HIV-CNS invasion and NeuroAIDS. J Neurosci, 2006. 26(4): p. 1098-106.

12. Thompson, K.A., et al., Brain cell reservoirs of latent virus in presymptomatic HIVinfected individuals. Am J Pathol, 2011. 179(4): p. 1623-9.

13. Ances, B.M. and R.J. Ellis, Dementia and neurocognitive disorders due to HIV-1 infection. Semin Neurol, 2007. 27(1): p. 86-92.

14. Gendelman, H.E., et al., The neuropathogenesis of the AIDS dementia complex. AIDS, 1997. 11 Suppl A: p. S35-45.

15. Strathdee, S.A. and J.K. Stockman, Epidemiology of HIV among injecting and noninjecting drug users: current trends and implications for interventions. Curr HIV/AIDS Rep, 2010. 7(2): p. 99-106.

16. Kyei, G.B., et al., Autophagy pathway intersects with HIV-1 biosynthesis and regulates viral yields in macrophages. J Cell Biol, 2009. 186(2): p. 255-68.

17. Klionsky, D.J., Autophagy revisited: a conversation with Christian de Duve. Autophagy, 2008. 4(6): p. 740-3. 
18. Hruban, Z., et al., Focal cytoplasmic degradation. Am J Pathol, 1963. 42: p. 65783.

19. Deter, R.L., P. Baudhuin, and C. De Duve, Participation of lysosomes in cellular autophagy induced in rat liver by glucagon. J Cell Biol, 1967. 35(2): p. C11-6.

20. Deter, R.L. and C. De Duve, Influence of glucagon, an inducer of cellular autophagy, on some physical properties of rat liver lysosomes. J Cell Biol, 1967. 33(2): p. 437-49.

21. Kaur, J. and J. Debnath, Autophagy at the crossroads of catabolism and anabolism. Nat Rev Mol Cell Biol, 2015. 16(8): p. 461-72.

22. Cesen, M.H., et al., Lysosomal pathways to cell death and their therapeutic applications. Exp Cell Res, 2012. 318(11): p. 1245-51.

23. Espert, L., et al., Differential role of autophagy in CD4 T cells and macrophages during X4 and R5 HIV-1 infection. PLoS One, 2009. 4(6): p. e5787.

24. Gentile, M., et al., Determination of the size of HIV using adenovirus type 2 as an internal length marker. J Virol Methods, 1994. 48(1): p. 43-52.

25. Li, G. and E. De Clercq, HIV Genome-Wide Protein Associations: a Review of 30 Years of Research. Microbiol Mol Biol Rev, 2016. 80(3): p. 679-731.

26. Lu, K., X. Heng, and M.F. Summers, Structural determinants and mechanism of HIV-1 genome packaging. J Mol Biol, 2011. 410(4): p. 609-33.

27. Frankel, A.D. and J.A. Young, HIV-1: fifteen proteins and an RNA. Annu Rev Biochem, 1998. 67: p. 1-25.

28. Karacostas, V., et al., Overexpression of the HIV-1 gag-pol polyprotein results in intracellular activation of HIV-1 protease and inhibition of assembly and budding of virus-like particles. Virology, 1993. 193(2): p. 661-71.

29. King, S.R., HIV: virology and mechanisms of disease. Ann Emerg Med, 1994. 24(3): p. 443-9.

30. Bagashev, A. and B.E. Sawaya, Roles and functions of HIV-1 Tat protein in the CNS: an overview. Virol J, 2013. 10: p. 358.

31. Debaisieux, S., et al., The ins and outs of HIV-1 Tat. Traffic, 2012. 13(3): p. 35563.

32. Feng, S. and E.C. Holland, HIV-1 tat trans-activation requires the loop sequence within tar. Nature, 1988. 334(6178): p. 165-7.

33. Mujeeb, A., et al., NMR structure of a biologically active peptide containing the RNA-binding domain of human immunodeficiency virus type 1 Tat. Proc Natl Acad Sci U S A, 1994. 91(17): p. 8248-52. 
34. Jones, K.A. and B.M. Peterlin, Control of RNA initiation and elongation at the HIV1 promoter. Annu Rev Biochem, 1994. 63: p. 717-43.

35. Wei, P., et al., A novel CDK9-associated C-type cyclin interacts directly with HIV1 Tat and mediates its high-affinity, loop-specific binding to TAR RNA. Cell, 1998. 92(4): p. 451-62.

36. Bayer, P., et al., Structural studies of HIV-1 Tat protein. J Mol Biol, 1995. 247(4): p. 529-35.

37. Vogel, B.E., et al., A novel integrin specificity exemplified by binding of the alpha $v$ beta 5 integrin to the basic domain of the HIV Tat protein and vitronectin. $\mathrm{J}$ Cell Biol, 1993. 121(2): p. 461-8.

38. Green, M. and P.M. Loewenstein, Autonomous functional domains of chemically synthesized human immunodeficiency virus tat trans-activator protein. Cell, 1988. 55(6): p. 1179-88.

39. Chang, H.C., et al., HIV-1 Tat protein exits from cells via a leaderless secretory pathway and binds to extracellular matrix-associated heparan sulfate proteoglycans through its basic region. AIDS, 1997. 11(12): p. 1421-31.

40. Tardieu, M., et al., Human immunodeficiency virus type 1-infected monocytic cells can destroy human neural cells after cell-to-cell adhesion. Ann Neurol, 1992. 32(1): p. 11-7.

41. Navia, B.A., B.D. Jordan, and R.W. Price, The AIDS dementia complex: I. Clinical features. Ann Neurol, 1986. 19(6): p. 517-24.

42. Navia, B.A. and R.W. Price, The acquired immunodeficiency syndrome dementia complex as the presenting or sole manifestation of human immunodeficiency virus infection. Arch Neurol, 1987. 44(1): p. 65-9.

43. Rosca, E.C., et al., HIV-associated neurocognitive disorders: a historical review. Neurologist, 2012. 18(2): p. 64-7.

44. Ragin, A.B., et al., Structural brain alterations can be detected early in HIV infection. Neurology, 2012. 79(24): p. 2328-34.

45. Ragin, A.B., et al., Brain alterations within the first 100 days of HIV infection. Ann Clin Transl Neurol, 2015. 2(1): p. 12-21.

46. Hawkins, C.P., et al., Pathological findings correlated with MRI in HIV infection. Neuroradiology, 1993. 35(4): p. 264-8.

47. Gonzalez-Scarano, F. and J. Martin-Garcia, The neuropathogenesis of AIDS. Nat Rev Immunol, 2005. 5(1): p. 69-81. 
48. Ances, B.M. and D.A. Hammoud, Neuroimaging of HIV-associated neurocognitive disorders (HAND). Curr Opin HIV AIDS, 2014. 9(6): p. 545-51.

49. McArthur, J.C. and B.J. Brew, HIV-associated neurocognitive disorders: is there a hidden epidemic? AIDS, 2010. 24(9): p. 1367-70.

50. Maschke, M., et al., Incidence and prevalence of neurological disorders associated with HIV since the introduction of highly active antiretroviral therapy (HAART). J Neurol Neurosurg Psychiatry, 2000. 69(3): p. 376-80.

51. Saylor, D., et al., HIV-associated neurocognitive disorder - pathogenesis and prospects for treatment. Nat Rev Neurol, 2016. 12(5): p. 309.

52. Nath, A., Pathobiology of human immunodeficiency virus dementia. Semin Neurol, 1999. 19(2): p. 113-27.

53. Resnick, L., et al., Early penetration of the blood-brain-barrier by HIV. Neurology, 1988. 38(1): p. 9-14.

54. Kaul, M., G.A. Garden, and S.A. Lipton, Pathways to neuronal injury and apoptosis in HIV-associated dementia. Nature, 2001. 410(6831): p. 988-94.

55. Rock, R.B., et al., Role of microglia in central nervous system infections. Clin Microbiol Rev, 2004. 17(4): p. 942-64, table of contents.

56. Kramer-Hammerle, S., et al., Cells of the central nervous system as targets and reservoirs of the human immunodeficiency virus. Virus Res, 2005. 111(2): p. 194213.

57. Ensoli, B., et al., Release, uptake, and effects of extracellular human immunodeficiency virus type 1 Tat protein on cell growth and viral transactivation. J Virol, 1993. 67(1): p. 277-87.

58. Hudson, L., et al., Detection of the human immunodeficiency virus regulatory protein tat in CNS tissues. J Neurovirol, 2000. 6(2): p. 145-55.

59. Bachani, M., et al., Detection of anti-tat antibodies in CSF of individuals with HIVassociated neurocognitive disorders. J Neurovirol, 2013. 19(1): p. 82-8.

60. D'Aversa, T.G., K.O. Yu, and J.W. Berman, Expression of chemokines by human fetal microglia after treatment with the human immunodeficiency virus type 1 protein Tat. J Neurovirol, 2004. 10(2): p. 86-97.

61. Perez-Alvarez, A. and A. Araque, Astrocyte-neuron interaction at tripartite synapses. Curr Drug Targets, 2013. 14(11): p. 1220-4.

62. Rao, V.R., A.P. Ruiz, and V.R. Prasad, Viral and cellular factors underlying neuropathogenesis in HIV associated neurocognitive disorders (HAND). AIDS research and therapy, 2014. 11: p. 13. 
63. Gorry, P.R., et al., Astrocyte infection by HIV-1: mechanisms of restricted virus replication, and role in the pathogenesis of HIV-1-associated dementia. Curr HIV Res, 2003. 1(4): p. 463-73.

64. Ranki, A., et al., Abundant expression of HIV Nef and Rev proteins in brain astrocytes in vivo is associated with dementia. AIDS, 1995. 9(9): p. 1001-8.

65. Conant, K., et al., Induction of monocyte chemoattractant protein-1 in HIV-1 Tatstimulated astrocytes and elevation in AIDS dementia. Proc Natl Acad Sci U S A, 1998. 95(6): p. 3117-21.

66. Nath, A., et al., Transient exposure to HIV-1 Tat protein results in cytokine production in macrophages and astrocytes. $A$ hit and run phenomenon. J Biol Chem, 1999. 274(24): p. 17098-102.

67. Liu, X., et al., Human immunodeficiency virus type 1 (HIV-1) tat induces nitric-oxide synthase in human astroglia. J Biol Chem, 2002. 277(42): p. 39312-9.

68. Eugenin, E.A., et al., Human immunodeficiency virus infection of human astrocytes disrupts blood-brain barrier integrity by a gap junction-dependent mechanism. J Neurosci, 2011. 31(26): p. 9456-65.

69. Fine, S.M., et al., Tumor necrosis factor alpha inhibits glutamate uptake by primary human astrocytes. Implications for pathogenesis of HIV-1 dementia. J Biol Chem, 1996. 271(26): p. 15303-6.

70. Holden, C.P., et al., Role of $\mathrm{Na}+/ \mathrm{H}+$ exchangers, excitatory amino acid receptors and voltage-operated Ca2+ channels in human immunodeficiency virus type 1 gp120-mediated increases in intracellular Ca2+ in human neurons and astrocytes. Neuroscience, 1999. 91(4): p. 1369-78.

71. Meucci, O., et al., Expression of CX3CR1 chemokine receptors on neurons and their role in neuronal survival. Proc Natl Acad Sci U S A, 2000. 97(14): p. 8075-80.

72. Everall, I., P. Luthert, and P. Lantos, A review of neuronal damage in human immunodeficiency virus infection: its assessment, possible mechanism and relationship to dementia. J Neuropathol Exp Neurol, 1993. 52(6): p. 561-6.

73. Gendelman, H.E., et al., The neuropathogenesis of HIV-1 infection. J Leukoc Biol, 1994. 56(3): p. 389-98.

74. Lannuzel, A., et al., HIV-1 envelope proteins gp120 and gp160 potentiate NMDAinduced $[\mathrm{Ca} 2+] i$ increase, alter $[\mathrm{Ca} 2+] i$ homeostasis and induce neurotoxicity in human embryonic neurons. Eur J Neurosci, 1995. 7(11): p. 2285-93.

75. Nath, A., et al., Identification of a human immunodeficiency virus type 1 Tat epitope that is neuroexcitatory and neurotoxic. J Virol, 1996. 70(3): p. 1475-80.

76. Kaul, M. and S.A. Lipton, Chemokines and activated macrophages in HIV gp120induced neuronal apoptosis. Proc Natl Acad Sci U S A, 1999. 96(14): p. 8212-6. 
77. Adle-Biassette, H., et al., Neuronal apoptosis in HIV infection in adults. Neuropathol Appl Neurobiol, 1995. 21(3): p. 218-27.

78. Pandhare, J. and C. Dash, A prospective on drug abuse-associated epigenetics and HIV-1 replication. Life Sci, 2011. 88(21-22): p. 995-9.

79. Cao, L., et al., Role of Autophagy in HIV Pathogenesis and Drug Abuse. Mol Neurobiol, 2016.

80. Samikkannu, T., et al., HIV-1 gp120 and morphine induced oxidative stress: role in cell cycle regulation. Front Microbiol, 2015. 6: p. 614.

81. Mahajan, S.D., et al., Tight junction regulation by morphine and HIV-1 tat modulates blood-brain barrier permeability. J Clin Immunol, 2008. 28(5): p. 52841.

82. Smith, D.B., P. Simmonds, and J.E. Bell, Brain viral burden, neuroinflammation and neurodegeneration in HAART-treated HIV positive injecting drug users. J Neurovirol, 2014. 20(1): p. 28-38.

83. Anthony, I.C., et al., The effects of illicit drugs on the HIV infected brain. Front Biosci, 2008. 13: p. 1294-307.

84. Byrd, D., et al., Impact of opiate addiction on neuroinflammation in HIV. J Neurovirol, 2012. 18(5): p. 364-73.

85. Arango, J.C., et al., Does drug abuse influence the microglial response in AIDS and HIV encephalitis? AIDS, 2004. 18 Suppl 1: p. S69-74.

86. Hauser, K.F., et al., Opiate drug use and the pathophysiology of neuroAIDS. Curr HIV Res, 2012. 10(5): p. 435-52.

87. Hauser, K.F. and P.E. Knapp, Interactions of HIV and drugs of abuse: the importance of glia, neural progenitors, and host genetic factors. Int Rev Neurobiol, 2014. 118: p. 231-313.

88. Ouzzine, M., et al., The UDP-glucuronosyltransferases of the blood-brain barrier: their role in drug metabolism and detoxication. Front Cell Neurosci, 2014. 8: p. 349.

89. Smith, H.S., Opioid metabolism. Mayo Clin Proc, 2009. 84(7): p. 613-24.

90. Arvidsson, U., et al., Distribution and targeting of a mu-opioid receptor (MOR1) in brain and spinal cord. J Neurosci, 1995. 15(5 Pt 1): p. 3328-41.

91. Peng, J., S. Sarkar, and S.L. Chang, Opioid receptor expression in human brain and peripheral tissues using absolute quantitative real-time RT-PCR. Drug Alcohol Depend, 2012. 124(3): p. 223-8. 
92. Pasternak, G.W. and Y.X. Pan, Mu opioids and their receptors: evolution of a concept. Pharmacol Rev, 2013. 65(4): p. 1257-317.

93. Yekkirala, A.S., A.E. Kalyuzhny, and P.S. Portoghese, Standard opioid agonists activate heteromeric opioid receptors: evidence for morphine and [d-Ala(2)MePhe(4)-Glyol(5)]enkephalin as selective mu-delta agonists. ACS Chem Neurosci, 2010. 1(2): p. 146-54.

94. Dhawan, B.N., et al., International Union of Pharmacology. XII. Classification of opioid receptors. Pharmacol Rev, 1996. 48(4): p. 567-92.

95. Wise, R.A. and P.P. Rompre, Brain dopamine and reward. Annu Rev Psychol, 1989. 40: p. 191-225.

96. Lutz, P.E. and B.L. Kieffer, Opioid receptors: distinct roles in mood disorders. Trends Neurosci, 2013. 36(3): p. 195-206.

97. Al-Hasani, R. and M.R. Bruchas, Molecular mechanisms of opioid receptordependent signaling and behavior. Anesthesiology, 2011. 115: p. 1363-81.

98. Stein, C., M. Schafer, and H. Machelska, Attacking pain at its source: new perspectives on opioids. Nat Med, 2003. 9(8): p. 1003-8.

99. Anthony, I.C., et al., Predisposition to accelerated Alzheimer-related changes in the brains of human immunodeficiency virus negative opiate abusers. Brain, 2010. 133(Pt 12): p. 3685-98.

100. Bruce-Keller, A.J., et al., Morphine causes rapid increases in glial activation and neuronal injury in the striatum of inducible HIV-1 Tat transgenic mice. Glia, 2008. 56(13): p. 1414-27.

101. Gurwell, J.A., et al., Synergistic neurotoxicity of opioids and human immunodeficiency virus-1 Tat protein in striatal neurons in vitro. Neuroscience, 2001. 102(3): p. 555-63.

102. Zou, S., et al., Morphine potentiates neurodegenerative effects of HIV-1 Tat through actions at mu-opioid receptor-expressing glia. Brain, 2011. 134(Pt 12): p. 3616-31.

103. Steele, A.D., E.E. Henderson, and T.J. Rogers, Mu-opioid modulation of HIV-1 coreceptor expression and HIV-1 replication. Virology, 2003. 309(1): p. 99-107.

104. Sorrell, M.E. and K.F. Hauser, Ligand-gated purinergic receptors regulate HIV-1 Tat and morphine related neurotoxicity in primary mouse striatal neuron-glia cocultures. J Neuroimmune Pharmacol, 2014. 9(2): p. 233-44.

105. El-Hage, N., et al., A novel bivalent HIV-1 entry inhibitor reveals fundamental differences in CCR5- $\mu$-opioid receptor interactions between human astroglia and microglia. AIDS (London, England), 2013. 27: p. 2181-90. 
106. Hauser, K.F., et al., HIV-1 neuropathogenesis: glial mechanisms revealed through substance abuse. J Neurochem, 2007. 100(3): p. 567-86.

107. Turchan-Cholewo, J., et al., Morphine and HIV-Tat increase microglial-free radical production and oxidative stress: possible role in cytokine regulation. J Neurochem, 2009. 108(1): p. 202-15.

108. Feng, Y., et al., The machinery of macroautophagy. Cell Res, 2014. 24(1): p. 2441.

109. Tsukada, M. and Y. Ohsumi, Isolation and characterization of autophagy-defective mutants of Saccharomyces cerevisiae. FEBS Lett, 1993. 333(1-2): p. 169-74.

110. Thumm, M., et al., Isolation of autophagocytosis mutants of Saccharomyces cerevisiae. FEBS Lett, 1994. 349(2): p. 275-80.

111. Kim, J., et al., AMPK and $m T O R$ regulate autophagy through direct phosphorylation of Ulk1. Nat Cell Biol, 2011. 13(2): p. 132-41.

112. Mizushima, N., The role of the Atg1/ULK1 complex in autophagy regulation. Curr Opin Cell Biol, 2010. 22(2): p. 132-9.

113. Kundu, M., ULK1, mammalian target of rapamycin, and mitochondria: linking nutrient availability and autophagy. Antioxid Redox Signal, 2011. 14(10): p. 19538.

114. Kim, J. and K.L. Guan, Regulation of the autophagy initiating kinase ULK1 by nutrients: roles of mTORC1 and AMPK. Cell Cycle, 2011. 10(9): p. 1337-8.

115. Hosokawa, N., et al., Nutrient-dependent mTORC1 association with the ULK1Atg13-FIP200 complex required for autophagy. Mol Biol Cell, 2009. 20(7): p. 198191.

116. Itakura, E. and N. Mizushima, Characterization of autophagosome formation site by a hierarchical analysis of mammalian Atg proteins. Autophagy, 2010. 6(6): p. 764-76.

117. Pattingre, S., et al., BCl-2 antiapoptotic proteins inhibit Beclin 1-dependent autophagy. Cell, 2005. 122(6): p. 927-39.

118. Russell, R.C., et al., ULK1 induces autophagy by phosphorylating Beclin-1 and activating VPS34 lipid kinase. Nat Cell Biol, 2013. 15(7): p. 741-50.

119. Axe, E.L., et al., Autophagosome formation from membrane compartments enriched in phosphatidylinositol 3-phosphate and dynamically connected to the endoplasmic reticulum. J Cell Biol, 2008. 182(4): p. 685-701.

120. Geng, J. and D.J. Klionsky, The Atg8 and Atg12 ubiquitin-like conjugation systems in macroautophagy. 'Protein modifications: beyond the usual suspects' review series. EMBO Rep, 2008. 9(9): p. 859-64. 
121. Mari, M., et al., An Atg9-containing compartment that functions in the early steps of autophagosome biogenesis. J Cell Biol, 2010. 190(6): p. 1005-22.

122. Puri, C., et al., ATG16L1 meets ATG9 in recycling endosomes: additional roles for the plasma membrane and endocytosis in autophagosome biogenesis. Autophagy, 2014. 10(1): p. 182-4.

123. Puri, C., et al., Diverse autophagosome membrane sources coalesce in recycling endosomes. Cell, 2013. 154(6): p. 1285-99.

124. Tanida, I., et al., HsAtg4B/HsApg4B/autophagin-1 cleaves the carboxyl termini of three human Atg8 homologues and delipidates microtubule-associated protein light chain 3- and GABAA receptor-associated protein-phospholipid conjugates. J Biol Chem, 2004. 279(35): p. 36268-76.

125. Fujita, N., et al., An Atg4B mutant hampers the lipidation of LC3 paralogues and causes defects in autophagosome closure. Mol Biol Cell, 2008. 19(11): p. 4651-9.

126. Weidberg, H., et al., LC3 and GATE-16/GABARAP subfamilies are both essential yet act differently in autophagosome biogenesis. EMBO J, 2010. 29(11): p. 1792802.

127. Kabeya, Y., et al., LC3, GABARAP and GATE16 localize to autophagosomal membrane depending on form-Il formation. J Cell Sci, 2004. 117(Pt 13): p. 280512.

128. Weidberg, H., E. Shvets, and Z. Elazar, Biogenesis and cargo selectivity of autophagosomes. Annu Rev Biochem, 2011. 80: p. 125-56.

129. Mizushima, N., Autophagy: process and function. Genes Dev, 2007. 21(22): p. 2861-73.

130. Shaid, S., et al., Ubiquitination and selective autophagy. Cell Death Differ, 2013. 20(1): p. 21-30.

131. Pankiv, S., et al., p62/SQSTM1 binds directly to Atg8/LC3 to facilitate degradation of ubiquitinated protein aggregates by autophagy. J Biol Chem, 2007. 282(33): p. 24131-45.

132. Moscat, J., M.T. Diaz-Meco, and M.W. Wooten, Signal integration and diversification through the p62 scaffold protein. Trends Biochem Sci, 2007. 32(2): p. $95-100$.

133. Mizushima, N., et al., Dissection of autophagosome formation using Apg5-deficient mouse embryonic stem cells. J Cell Biol, 2001. 152(4): p. 657-68.

134. Yoshimori, T. and T. Noda, Toward unraveling membrane biogenesis in mammalian autophagy. Curr Opin Cell Biol, 2008. 20(4): p. 401-7. 
135. Nakatogawa, H., et al., Dynamics and diversity in autophagy mechanisms: lessons from yeast. Nat Rev Mol Cell Biol, 2009. 10(7): p. 458-67.

136. Qu, X., et al., Promotion of tumorigenesis by heterozygous disruption of the beclin 1 autophagy gene. J Clin Invest, 2003. 112(12): p. 1809-20.

137. Yue, Z., et al., Beclin 1, an autophagy gene essential for early embryonic development, is a haploinsufficient tumor suppressor. Proc Natl Acad Sci U S A, 2003. 100(25): p. 15077-82.

138. Wirawan, E., et al., Beclin 1: a role in membrane dynamics and beyond. Autophagy, 2012. 8(1): p. 6-17.

139. Liang, C., et al., Autophagic and tumour suppressor activity of a novel Beclin1binding protein UVRAG. Nat Cell Biol, 2006. 8(7): p. 688-99.

140. Furuya, N., et al., The evolutionarily conserved domain of Beclin 1 is required for Vps34 binding, autophagy and tumor suppressor function. Autophagy, 2005. 1(1): p. 46-52.

141. Maiuri, M.C., et al., Functional and physical interaction between $B c l-X(L)$ and a BH3-like domain in Beclin-1. EMBO J, 2007. 26(10): p. 2527-39.

142. Levine, B., S. Sinha, and G. Kroemer, Bcl-2 family members: dual regulators of apoptosis and autophagy. Autophagy, 2008. 4(5): p. 600-6.

143. Mizushima, N. and M. Komatsu, Autophagy: renovation of cells and tissues. Cell, 2011. 147(4): p. 728-41.

144. Boya, P., F. Reggiori, and P. Codogno, Emerging regulation and functions of autophagy. Nat Cell Biol, 2013. 15(7): p. 713-20.

145. Ezaki, J., et al., Liver autophagy contributes to the maintenance of blood glucose and amino acid levels. Autophagy, 2011. 7(7): p. 727-36.

146. Singh, R., et al., Autophagy regulates lipid metabolism. Nature, 2009. 458(7242): p. 1131-5.

147. White, E., Deconvoluting the context-dependent role for autophagy in cancer. Nat Rev Cancer, 2012. 12(6): p. 401-10.

148. Eng, C.H. and R.T. Abraham, Glutaminolysis yields a metabolic by-product that stimulates autophagy. Autophagy, 2010. 6(7): p. 968-70.

149. Di Malta, C., et al., Autophagy in astrocytes: a novel culprit in lysosomal storage disorders. Autophagy, 2012. 8(12): p. 1871-2.

150. Di Malta, C., et al., Astrocyte dysfunction triggers neurodegeneration in a lysosomal storage disorder. Proc Natl Acad Sci U S A, 2012. 109(35): p. E233442. 
151. Deretic, V., S. Jiang, and N. Dupont, Autophagy intersections with conventional and unconventional secretion in tissue development, remodeling and inflammation. Trends Cell Biol, 2012. 22(8): p. 397-406.

152. Cebollero, E., F. Reggiori, and C. Kraft, Reticulophagy and ribophagy: regulated degradation of protein production factories. Int J Cell Biol, 2012. 2012: p. 182834.

153. Youle, R.J. and D.P. Narendra, Mechanisms of mitophagy. Nat Rev Mol Cell Biol, 2011. 12(1): p. 9-14.

154. Kroemer, G., G. Marino, and B. Levine, Autophagy and the integrated stress response. Mol Cell, 2010. 40(2): p. 280-93.

155. Ryter, S.W., S.M. Cloonan, and A.M. Choi, Autophagy: a critical regulator of cellular metabolism and homeostasis. Mol Cells, 2013. 36(1): p. 7-16.

156. Lamark, T. and T. Johansen, Aggrephagy: selective disposal of protein aggregates by macroautophagy. Int J Cell Biol, 2012. 2012: p. 736905.

157. Komatsu, M., et al., Impairment of starvation-induced and constitutive autophagy in Atg7-deficient mice. J Cell Biol, 2005. 169(3): p. 425-34.

158. Hara, T., et al., Suppression of basal autophagy in neural cells causes neurodegenerative disease in mice. Nature, 2006. 441(7095): p. 885-9.

159. Nakai, A., et al., The role of autophagy in cardiomyocytes in the basal state and in response to hemodynamic stress. Nat Med, 2007. 13(5): p. 619-24.

160. Maiuri, M.C., et al., Self-eating and self-killing: crosstalk between autophagy and apoptosis. Nat Rev Mol Cell Biol, 2007. 8(9): p. 741-52.

161. Levine, B. and J. Yuan, Autophagy in cell death: an innocent convict? J Clin Invest, 2005. 115(10): p. 2679-88.

162. Shimizu, S., et al., Role of Bcl-2 family proteins in a non-apoptotic programmed cell death dependent on autophagy genes. Nat Cell Biol, 2004. 6(12): p. 1221-8.

163. Yonekawa, T. and A. Thorburn, Autophagy and cell death. Essays Biochem, 2013. 55: p. 105-17.

164. Deretic, V. and B. Levine, Autophagy, immunity, and microbial adaptations. Cell Host Microbe, 2009. 5(6): p. 527-49.

165. Levine, B., N. Mizushima, and H.W. Virgin, Autophagy in immunity and inflammation. Nature, 2011. 469(7330): p. 323-35.

166. Levine, B., Eating oneself and uninvited guests: autophagy-related pathways in cellular defense. Cell, 2005. 120(2): p. 159-62. 
167. Shi, C.S., et al., Activation of autophagy by inflammatory signals limits IL-1beta production by targeting ubiquitinated inflammasomes for destruction. Nat Immunol, 2012. 13(3): p. 255-63.

168. Schmid, D., M. Pypaert, and C. Munz, Antigen-loading compartments for major histocompatibility complex class II molecules continuously receive input from autophagosomes. Immunity, 2007. 26(1): p. 79-92.

169. Byrne, B.G., et al., Inflammasome components coordinate autophagy and pyroptosis as macrophage responses to infection. MBio, 2013. 4(1): p. e00620-12.

170. Doitsh, G., et al., Cell death by pyroptosis drives CD4 T-cell depletion in HIV-1 infection. Nature, 2014. 505(7484): p. 509-14.

171. Blanchet, F.P., et al., Human immunodeficiency virus-1 inhibition of immunoamphisomes in dendritic cells impairs early innate and adaptive immune responses. Immunity, 2010. 32(5): p. 654-69.

172. Li, J.C., et al., HIV-1 trans-activator protein dysregulates IFN-gamma signaling and contributes to the suppression of autophagy induction. AIDS, 2011. 25(1): p. 1525.

173. Van Grol, J., et al., HIV-1 inhibits autophagy in bystander macrophage/monocytic cells through Src-Akt and STAT3. PLoS One, 2010. 5(7): p. e11733.

174. Dinkins, C., M. Pilli, and J.H. Kehrl, Roles of autophagy in HIV infection. Immunol Cell Biol, 2015. 93(1): p. 11-7.

175. Espert, L., et al., Autophagy is involved in T cell death after binding of HIV-1 envelope proteins to CXCR4. J Clin Invest, 2006. 116(8): p. 2161-72.

176. Espert, L., B. Beaumelle, and I. Vergne, Autophagy in Mycobacterium tuberculosis and HIV infections. Front Cell Infect Microbiol, 2015. 5: p. 49.

177. Borel, S., et al., HIV-1 viral infectivity factor interacts with microtubule-associated protein light chain 3 and inhibits autophagy. AIDS, 2015. 29(3): p. 275-86.

178. Sagnier, S., et al., Autophagy restricts HIV-1 infection by selectively degrading Tat in CD4+ T lymphocytes. J Virol, 2015. 89(1): p. 615-25.

179. Nardacci, R., et al., Autophagy plays an important role in the containment of HIV1 in nonprogressor-infected patients. Autophagy, 2014. 10(7): p. 1167-78.

180. Wong, E. and A.M. Cuervo, Autophagy gone awry in neurodegenerative diseases. Nat Neurosci, 2010. 13(7): p. 805-11.

181. Mizushima, N., et al., In vivo analysis of autophagy in response to nutrient starvation using transgenic mice expressing a fluorescent autophagosome marker. Mol Biol Cell, 2004. 15(3): p. 1101-11. 
182. Mizushima, N., Physiological functions of autophagy. Curr Top Microbiol Immunol, 2009. 335: p. 71-84.

183. Winslow, A.R. and D.C. Rubinsztein, Autophagy in neurodegeneration and development. Biochim Biophys Acta, 2008. 1782(12): p. 723-9.

184. Komatsu, M., et al., Loss of autophagy in the central nervous system causes neurodegeneration in mice. Nature, 2006. 441(7095): p. 880-4.

185. Zhou, D., E. Masliah, and S.A. Spector, Autophagy is increased in postmortem brains of persons with HIV-1-associated encephalitis. J Infect Dis, 2011. 203(11): p. 1647-57.

186. Fields, J., et al., Age-dependent molecular alterations in the autophagy pathway in HIVE patients and in a gp120 tg mouse model: reversal with beclin-1 gene transfer. J Neurovirol, 2013. 19(1): p. 89-101.

187. Dever, S.M., et al., Differing roles of autophagy in HIV-associated neurocognitive impairment and encephalitis with implications for morphine co-exposure. Front Microbiol, 2015. 6: p. 653.

188. Mehla, R. and A. Chauhan, HIV-1 differentially modulates autophagy in neurons and astrocytes. J Neuroimmunol, 2015. 285: p. 106-18.

189. Alirezaei, M., et al., Disruption of neuronal autophagy by infected microglia results in neurodegeneration. PLoS One, 2008. 3(8): p. e2906.

190. Alirezaei, M., W.B. Kiosses, and H.S. Fox, Decreased neuronal autophagy in HIV dementia: a mechanism of indirect neurotoxicity. Autophagy, 2008. 4(7): p. 963-6.

191. Fields, J., et al., HIV-1 Tat alters neuronal autophagy by modulating autophagosome fusion to the lysosome: implications for HIV-associated neurocognitive disorders. J Neurosci, 2015. 35(5): p. 1921-38.

192. El-Hage, N., et al., HIV-1 and Morphine Regulation of Autophagy in Microglia: Limited Interactions in the Context of HIV-1 Infection and Opioid Abuse. Journal of virology, 2015. 89: p. 1024-35.

193. Bruno, A.P., et al., HIV-1 Tat protein induces glial cell autophagy through enhancement of BAG3 protein levels. Cell Cycle, 2014. 13(23): p. 3640-4.

194. Saribas, A.S., K. Khalili, and I.K. Sariyer, Dysregulation of autophagy by HIV-1 Nef in human astrocytes. Cell Cycle, 2015. 14(18): p. 2899-904.

195. Feng, Y.M., et al., Decreased mitochondrial DNA copy number in the hippocampus and peripheral blood during opiate addiction is mediated by autophagy and can be salvaged by melatonin. Autophagy, 2013. 9(9): p. 1395-406. 
196. Zhao, L., et al., Morphine induces Beclin 1-and ATG5-dependent autophagy in human neuroblastoma SH-SY5Y cells and in the rat hippocampus. Autophagy, 2010. 6: p. 386-394.

197. Wan, J., et al., Morphine potentiates LPS-induced autophagy initiation but inhibits autophagosomal maturation through distinct TLR4-dependent and independent pathways. Acta Physiol (Oxf), 2015. 214(2): p. 189-99.

198. Letendre, S., Central nervous system complications in HIV disease: HIVassociated neurocognitive disorder. Top Antivir Med, 2011. 19(4): p. 137-42.

199. Jones, M., et al., Intraventricular injection of human immunodeficiency virus type 1 (HIV-1) tat protein causes inflammation, gliosis, apoptosis, and ventricular enlargement. J Neuropathol Exp Neurol, 1998. 57(6): p. 563-70.

200. Vogel, J., et al., The HIV tat gene induces dermal lesions resembling Kaposi's sarcoma in transgenic mice. Nature, 1988. 335(6191): p. 606-11.

201. Vellutini, C., et al., Development of lymphoid hyperplasia in transgenic mice expressing the HIV tat gene. AIDS Res Hum Retroviruses, 1995. 11(1): p. 21-9.

202. Garza, H.H., Jr., O. Prakash, and D.J. Carr, Aberrant regulation of cytokines in HIV-1 TAT72-transgenic mice. J Immunol, 1996. 156(10): p. 3631-7.

203. Choi, J., et al., Molecular mechanism of decreased glutathione content in human immunodeficiency virus type 1 Tat-transgenic mice. J Biol Chem, 2000. 275(5): p. 3693-8.

204. Fitting, S., et al., Interactive comorbidity between opioid drug abuse and HIV-1 Tat: chronic exposure augments spine loss and sublethal dendritic pathology in striatal neurons. Am J Pathol, 2010. 177(3): p. 1397-410.

205. Kim, B.O., et al., Neuropathologies in transgenic mice expressing human immunodeficiency virus type 1 Tat protein under the regulation of the astrocytespecific glial fibrillary acidic protein promoter and doxycycline. Am J Pathol, 2003. 162(5): p. 1693-707.

206. Carey, A.N., et al., Expression of HIV-Tat protein is associated with learning and memory deficits in the mouse. Behav Brain Res, 2012. 229(1): p. 48-56.

207. Fitting, S., et al., Synaptic dysfunction in the hippocampus accompanies learning and memory deficits in human immunodeficiency virus type-1 Tat transgenic mice. Biol Psychiatry, 2013. 73(5): p. 443-53.

208. Leibrand, C.R., et al., HIV-1 Tat disrupts blood-brain barrier integrity and increases phagocytic perivascular macrophages and microglia in the dorsal striatum of transgenic mice. Neurosci Lett, 2017. 640: p. 136-143.

209. Fitting, S., et al., Morphine efficacy is altered in conditional HIV-1 Tat transgenic mice. Eur J Pharmacol, 2012. 689(1-3): p. 96-103. 
210. Toggas, S.M., et al., Central nervous system damage produced by expression of the HIV-1 coat protein gp120 in transgenic mice. Nature, 1994. 367(6459): p. 18893.

211. Berrada, F., et al., Neuronal expression of human immunodeficiency virus type 1 env proteins in transgenic mice: distribution in the central nervous system and pathological alterations. J Virol, 1995. 69(11): p. 6770-8.

212. Okamoto, S., et al., HIV/gp120 decreases adult neural progenitor cell proliferation via checkpoint kinase-mediated cell-cycle withdrawal and G1 arrest. Cell Stem Cell, 2007. 1(2): p. 230-6.

213. Lee, M.H., et al., Rescue of adult hippocampal neurogenesis in a mouse model of HIV neurologic disease. Neurobiol Dis, 2011. 41(3): p. 678-87.

214. D'Hooge, R., et al., Age-related behavioural deficits in transgenic mice expressing the HIV-1 coat protein gp120. Eur J Neurosci, 1999. 11(12): p. 4398-402.

215. Maung, R., et al., Genetic knockouts suggest a critical role for HIV co-receptors in models of HIV gp120-induced brain injury. J Neuroimmune Pharmacol, 2012. 7(2): p. 306-18.

216. Toggas, S.M., E. Masliah, and L. Mucke, Prevention of HIV-1 gp120-induced neuronal damage in the central nervous system of transgenic mice by the NMDA receptor antagonist memantine. Brain Res, 1996. 706(2): p. 303-7.

217. Krucker, T., et al., Transgenic mice with cerebral expression of human immunodeficiency virus type-1 coat protein gp120 show divergent changes in short- and long-term potentiation in CA1 hippocampus. Neuroscience, 1998. 83(3): p. 691-700.

218. Vigorito, M., K.P. Connaghan, and S.L. Chang, The HIV-1 transgenic rat model of neuroHIV. Brain Behav Immun, 2015. 48: p. 336-49.

219. Kopp, J.B., et al., Cutaneous disorders and viral gene expression in HIV-1 transgenic mice. AIDS Res Hum Retroviruses, 1993. 9(3): p. 267-75.

220. Reid, W., et al., An HIV-1 transgenic rat that develops HIV-related pathology and immunologic dysfunction. Proc Natl Acad Sci U S A, 2001. 98(16): p. 9271-6.

221. Peng, J., et al., The HIV-1 transgenic rat as a model for HIV-1 infected individuals on HAART. J Neuroimmunol, 2010. 218(1-2): p. 94-101.

222. Moran, L.M., et al., Neurobehavioral alterations in HIV-1 transgenic rats: evidence for dopaminergic dysfunction. Exp Neurol, 2013. 239: p. 139-47.

223. Royal, W., 3rd, et al., Immune activation, viral gene product expression and neurotoxicity in the HIV-1 transgenic rat. J Neuroimmunol, 2012. 247(1-2): p. 1624. 
224. Mahajan, S.D., et al., Morphine regulates gene expression of alpha- and betachemokines and their receptors on astroglial cells via the opioid mu receptor. $\mathrm{J}$ Immunol, 2002. 169(7): p. 3589-99.

225. Borner, C., et al., Transcriptional regulation of the human mu-opioid receptor gene by interleukin-6. Mol Pharmacol, 2004. 66(6): p. 1719-26.

226. Chang, S.L., J.A. Beltran, and S. Swarup, Expression of the mu opioid receptor in the human immunodeficiency virus type 1 transgenic rat model. J Virol, 2007. 81(16): p. 8406-11.

227. Jaeger, L.B. and A. Nath, Modeling HIV-associated neurocognitive disorders in mice: new approaches in the changing face of HIV neuropathogenesis. Dis Model Mech, 2012. 5(3): p. 313-22.

228. Eggert, D., et al., Neuroprotective activities of CEP-1347 in models of neuroAIDS. J Immunol, 2010. 184(2): p. 746-56.

229. Persidsky, Y., et al., Human immunodeficiency virus encephalitis in SCID mice. Am J Pathol, 1996. 149(3): p. 1027-53.

230. Persidsky, Y. and H.E. Gendelman, Development of laboratory and animal model systems for HIV-1 encephalitis and its associated dementia. J Leukoc Biol, 1997. 62(1): p. 100-6.

231. Ito, M., et al., NOD/SCID/gamma(c)(null) mouse: an excellent recipient mouse model for engraftment of human cells. Blood, 2002. 100(9): p. 3175-82.

232. Shultz, L.D., et al., Human lymphoid and myeloid cell development in NOD/LtSzscid IL2R gamma null mice engrafted with mobilized human hemopoietic stem cells. J Immunol, 2005. 174(10): p. 6477-89.

233. Traggiai, E., et al., Development of a human adaptive immune system in cord blood cell-transplanted mice. Science, 2004. 304(5667): p. 104-7.

234. Akkina, R., et al., Improvements and Limitations of Humanized Mouse Models for HIV Research: NIH/NIAID "Meet the Experts" 2015 Workshop Summary. AIDS Res Hum Retroviruses, 2016. 32(2): p. 109-19.

235. van Maanen, M. and R.E. Sutton, Rodent models for HIV-1 infection and disease. Curr HIV Res, 2003. 1(1): p. 121-30.

236. Potash, M.J., et al., A mouse model for study of systemic HIV-1 infection, antiviral immune responses, and neuroinvasiveness. Proc Natl Acad Sci U S A, 2005. 102(10): p. 3760-5.

237. Jones, L.D., J.W. Jackson, and S.B. Maggirwar, Modeling HIV-1 Induced Neuroinflammation in Mice: Role of Platelets in Mediating Blood-Brain Barrier Dysfunction. PLoS One, 2016. 11(3): p. e0151702. 
238. Kelschenbach, J.L., et al., Mice chronically infected with chimeric HIV resist peripheral and brain superinfection: a model of protective immunity to HIV. J Neuroimmune Pharmacol, 2012. 7(2): p. 380-7.

239. Hayman, M., et al., Neurotoxicity of peptide analogues of the transactivating protein tat from Maedi-Visna virus and human immunodeficiency virus. Neuroscience, 1993. 53(1): p. 1-6.

240. Philippon, V., et al., The basic domain of the lentiviral Tat protein is responsible for damages in mouse brain: involvement of cytokines. Virology, 1994. 205(2): p. 51929.

241. Bansal, A.K., et al., Neurotoxicity of HIV-1 proteins gp120 and Tat in the rat striatum. Brain Res, 2000. 879(1-2): p. 42-9.

242. El-Hage, N., et al., HIV-1 Tat and opiate-induced changes in astrocytes promote chemotaxis of microglia through the expression of MCP-1 and alternative chemokines. Glia, 2006. 53(2): p. 132-46.

243. El-Hage, N., et al., CCR2 mediates increases in glial activation caused by exposure to HIV-1 Tat and opiates. J Neuroimmunol, 2006. 178(1-2): p. 9-16.

244. El-Hage, N., et al., CCL5/RANTES gene deletion attenuates opioid-induced increases in glial CCL2/MCP-1 immunoreactivity and activation in HIV-1 Tatexposed mice. J Neuroimmune Pharmacol, 2008. 3(4): p. 275-85.

245. Cann, G.M., et al., Developmental expression of LC3alpha and beta: absence of fibronectin or autophagy phenotype in LC3beta knockout mice. Dev Dyn, 2008. 237(1): p. 187-95.

246. Rodriguez, M., et al., Importance of Autophagy in Mediating Human Immunodeficiency Virus (HIV) and Morphine-Induced Metabolic Dysfunction and Inflammation in Human Astrocytes. Viruses, 2017. 9(8): p. 201.

247. Rodriguez, M., et al., Electro-Magnetic Nano-Particle Bound Beclin1 siRNA Crosses the Blood-Brain Barrier to Attenuate the Inflammatory Effects of HIV-1 Infection in Vitro. J Neuroimmune Pharmacol, 2016.

248. Hui, L., et al., Role of endolysosomes in HIV-1 Tat-induced neurotoxicity. ASN Neuro, 2012. 4(4): p. 243-52.

249. Pulliam, L., et al., Human immunodeficiency virus-infected macrophages produce soluble factors that cause histological and neurochemical alterations in cultured human brains. J Clin Invest, 1991. 87(2): p. 503-12.

250. Pocernich, C.B., et al., HIV-dementia, Tat-induced oxidative stress, and antioxidant therapeutic considerations. Brain Res Brain Res Rev, 2005. 50(1): p. 14-26. 
251. Haughey, N.J., et al., Involvement of inositol 1,4,5-trisphosphate-regulated stores of intracellular calcium in calcium dysregulation and neuron cell death caused by HIV-1 protein tat. J Neurochem, 1999. 73(4): p. 1363-74.

252. New, D.R., et al., Human immunodeficiency virus type 1 Tat protein induces death by apoptosis in primary human neuron cultures. J Neurovirol, 1997. 3(2): p. 16873.

253. Friedman, H., S. Pross, and T.W. Klein, Addictive drugs and their relationship with infectious diseases. FEMS Immunol Med Microbiol, 2006. 47(3): p. 330-42.

254. Klionsky, D.J., et al., A comprehensive glossary of autophagy-related molecules and processes (2nd edition). Autophagy, 2011. 7(11): p. 1273-94.

255. Kiriyama, Y. and H. Nochi, The Function of Autophagy in Neurodegenerative Diseases. Int J Mol Sci, 2015. 16(11): p. 26797-812.

256. Zhou, D. and S.A. Spector, Human immunodeficiency virus type-1 infection inhibits autophagy. AIDS (London, England), 2008. 22: p. 695-9.

257. Rodriguez, M., et al., Electro-Magnetic Nano-Particle Bound Beclin1 siRNA Crosses the Blood-Brain Barrier to Attenuate the Inflammatory Effects of HIV-1 Infection in Vitro. J Neuroimmune Pharmacol, 2017. 12(1): p. 120-132.

258. Rodriguez, M., et al., Intranasal drug delivery of small interfering RNA targeting Beclin1 encapsulated with polyethylenimine (PEI) in mouse brain to achieve HIV attenuation. Sci Rep, 2017. 7(1): p. 1862.

259. Mayne, M., et al., HIV-1 tat molecular diversity and induction of TNF-alpha: implications for HIV-induced neurological disease. Neuroimmunomodulation, 1998. 5(3-4): p. 184-92.

260. Kruman, II, A. Nath, and M.P. Mattson, HIV-1 protein Tat induces apoptosis of hippocampal neurons by a mechanism involving caspase activation, calcium overload, and oxidative stress. Exp Neurol, 1998. 154(2): p. 276-88.

261. El-Hage, N., et al., Synergistic increases in intracellular Ca2+, and the release of MCP-1, RANTES, and IL-6 by astrocytes treated with opiates and HIV-1 Tat. Glia, 2005. 50(2): p. 91-106.

262. McKnight, N.C., et al., Beclin 1 is required for neuron viability and regulates endosome pathways via the UVRAG-VPS34 complex. PLoS Genet, 2014. 10(10): p. e1004626.

263. Kabeya, Y., et al., LC3, a mammalian homologue of yeast Apg8p, is localized in autophagosome membranes after processing. EMBO J, 2000. 19(21): p. 5720-8.

264. El-Hage, N., et al., Morphine exacerbates HIV-1 Tat-induced cytokine production in astrocytes through convergent effects on $[\mathrm{Ca}(2+)](i), N F-k a p p a B$ trafficking and transcription. PLoS One, 2008. 3(12): p. e4093. 
265. Sun, Q., et al., Inflammasome and autophagy regulation - a two-way street. Mol Med, 2017. 23.

266. Deretic, V., T. Saitoh, and S. Akira, Autophagy in infection, inflammation and immunity. Nat Rev Immunol, 2013. 13(10): p. 722-37.

267. Salminen, A., K. Kaarniranta, and A. Kauppinen, Beclin 1 interactome controls the crosstalk between apoptosis, autophagy and inflammasome activation: impact on the aging process. Ageing Res Rev, 2013. 12(2): p. 520-34.

268. Eskelinen, E.L., Roles of LAMP-1 and LAMP-2 in lysosome biogenesis and autophagy. Mol Aspects Med, 2006. 27(5-6): p. 495-502.

269. Cornell-Bell, A.H., et al., Glutamate induces calcium waves in cultured astrocytes: long-range glial signaling. Science, 1990. 247(4941): p. 470-3.

270. Wang, X., T. Takano, and M. Nedergaard, Astrocytic calcium signaling: mechanism and implications for functional brain imaging. Methods Mol Biol, 2009. 489: p. 93-109.

271. Barger, S.W., et al., Glutamate release from activated microglia requires the oxidative burst and lipid peroxidation. J Neurochem, 2007. 101(5): p. 1205-13.

272. Norman, J.P., et al., HIV-1 Tat activates neuronal ryanodine receptors with rapid induction of the unfolded protein response and mitochondrial hyperpolarization. PLoS One, 2008. 3(11): p. e3731.

273. Haughey, N.J., et al., HIV-1 Tat through phosphorylation of NMDA receptors potentiates glutamate excitotoxicity. J Neurochem, 2001. 78(3): p. 457-67.

274. Kruman, II, et al., Evidence that Par-4 participates in the pathogenesis of HIV encephalitis. Am J Pathol, 1999. 155(1): p. 39-46.

275. D'Aversa, T.G., E.A. Eugenin, and J.W. Berman, NeuroAIDS: contributions of the human immunodeficiency virus-1 proteins Tat and gp120 as well as CD40 to microglial activation. J Neurosci Res, 2005. 81(3): p. 436-46.

276. Shi, B., et al., Neuronal apoptosis induced by HIV-1 Tat protein and TNF-alpha: potentiation of neurotoxicity mediated by oxidative stress and implications for HIV1 dementia. J Neurovirol, 1998. 4(3): p. 281-90.

277. Steiner, J., et al., Oxidative stress and therapeutic approaches in HIV dementia. Antioxid Redox Signal, 2006. 8(11-12): p. 2089-100.

278. Streit, W.J., S.A. Walter, and N.A. Pennell, Reactive microgliosis. Prog Neurobiol, 1999. 57(6): p. 563-81.

279. Droge, W., Free radicals in the physiological control of cell function. Physiol Rev, 2002. 82(1): p. 47-95. 
280. Kaul, N. and H.J. Forman, Activation of NF kappa B by the respiratory burst of macrophages. Free Radic Biol Med, 1996. 21(3): p. 401-5.

281. Olsen, B.B., T.H. Svenstrup, and B. Guerra, Downregulation of protein kinase CK2 induces autophagic cell death through modulation of the mTOR and MAPK signaling pathways in human glioblastoma cells. Int J Oncol, 2012. 41(6): p. 196776.

282. Fassina, L., et al., AUTOCOUNTER, an ImageJ JavaScript to analyze LC3B-GFP expression dynamics in autophagy-induced astrocytoma cells. Eur J Histochem, 2012. 56(4): p. e44.

283. Dolman, N.J., J.A. Kilgore, and M.W. Davidson, A review of reagents for fluorescence microscopy of cellular compartments and structures, part I: BacMam labeling and reagents for vesicular structures. Curr Protoc Cytom, 2013. Chapter 12: p. Unit 1230.

284. Bokhari, S.M., et al., Morphine enhances Tat-induced activation in murine microglia. J Neurovirol, 2009. 15(3): p. 219-28.

285. Campbell, G.R., et al., Human Immunodeficiency Virus Type 1 Nef Inhibits Autophagy through Transcription Factor EB Sequestration. PLoS Pathog, 2015. 11(6): p. e1005018.

286. Liu, Z., et al., ASPP2 Plays a Dual Role in gp120-Induced Autophagy and Apoptosis of Neuroblastoma Cells. Front Neurosci, 2017. 11: p. 150.

287. Mabrouk, K., et al., Lethal neurotoxicity in mice of the basic domains of HIV and SIV Rev proteins. Study of these regions by circular dichroism. FEBS Lett, 1991. 289(1): p. 13-7.

288. Ronaldson, P.T. and R. Bendayan, HIV-1 viral envelope glycoprotein gp120 triggers an inflammatory response in cultured rat astrocytes and regulates the functional expression of P-glycoprotein. Mol Pharmacol, 2006. 70(3): p. 1087-98.

289. Silverstein, P.S., et al., HIV-1 gp120 and drugs of abuse: interactions in the central nervous system. Curr HIV Res, 2012. 10(5): p. 369-83.

290. Trillo-Pazos, G., et al., Recombinant nef HIV-IIIB protein is toxic to human neurons in culture. Brain Res, 2000. 864(2): p. 315-26.

291. Conant, K., et al., Extracellular human immunodeficiency virus type 1 Tat protein is associated with an increase in both NF-kappa B binding and protein kinase $C$ activity in primary human astrocytes. J Virol, 1996. 70(3): p. 1384-9.

292. Liu, X. and A. Kumar, Differential signaling mechanism for HIV-1 Nef-mediated production of IL-6 and IL-8 in human astrocytes. Sci Rep, 2015. 5: p. 9867.

293. Si, Q., et al., Vpr- and Nef-dependent induction of RANTES/CCL5 in microglial cells. Virology, 2002. 301(2): p. 342-53. 
294. Robichaud, G.A. and L. Poulin, HIV type 1 nef gene inhibits tumor necrosis factor alpha-induced apoptosis and promotes cell proliferation through the action of MAPK and JNK in human glial cells. AIDS Res Hum Retroviruses, 2000. 16(18): p. $1959-65$.

295. Fiala, M., et al., Regulation of HIV-1 infection in astrocytes: expression of Nef, TNF-alpha and IL-6 is enhanced in coculture of astrocytes with macrophages. $\mathrm{J}$ Neurovirol, 1996. 2(3): p. 158-66.

296. Sami Saribas, A., et al., HIV-1 Nef is released in extracellular vesicles derived from astrocytes: evidence for Nef-mediated neurotoxicity. Cell Death Dis, 2017. 8(1): p. e2542.

297. Madrid, R., et al., Nef-induced alteration of the early/recycling endosomal compartment correlates with enhancement of HIV-1 infectivity. J Biol Chem, 2005. 280(6): p. 5032-44.

298. Leymarie, O., L. Lepont, and C. Berlioz-Torrent, Canonical and Non-Canonical Autophagy in HIV-1 Replication Cycle. Viruses, 2017. 9(10).

299. Turchan-Cholewo, J., et al., Cell-specific actions of HIV-Tat and morphine on opioid receptor expression in glia. J Neurosci Res, 2008. 86(9): p. 2100-10.

300. Patel, C.N., et al., Chronic opioid antagonist treatment selectively regulates trafficking and signaling proteins in mouse spinal cord. Synapse, 2003. 50(1): p. 67-76.

301. Rogers, T.J. and P.K. Peterson, Opioid G protein-coupled receptors: signals at the crossroads of inflammation. Trends Immunol, 2003. 24(3): p. 116-21.

302. Keith, D.E., et al., mu-Opioid receptor internalization: opiate drugs have differential effects on a conserved endocytic mechanism in vitro and in the mammalian brain. Mol Pharmacol, 1998. 53(3): p. 377-84.

303. Thoresen, S.B., et al., A phosphatidylinositol 3-kinase class III sub-complex containing VPS15, VPS34, Beclin 1, UVRAG and BIF-1 regulates cytokinesis and degradative endocytic traffic. Exp Cell Res, 2010. 316(20): p. 3368-78.

304. Itakura, E., et al., Beclin 1 forms two distinct phosphatidylinositol 3-kinase complexes with mammalian Atg14 and UVRAG. Mol Biol Cell, 2008. 19(12): p. 5360-72.

305. Engedal, N. and P.O. Seglen, Autophagy of cytoplasmic bulk cargo does not require LC3. Autophagy, 2016. 12(2): p. 439-41.

306. Yoshii, S.R. and N. Mizushima, Monitoring and Measuring Autophagy. Int J Mol Sci, 2017. 18(9).

307. Mizushima, N. and T. Yoshimori, How to interpret LC3 immunoblotting. Autophagy, 2007. 3(6): p. 542-5. 
308. Klionsky, D.J., et al., Guidelines for the use and interpretation of assays for monitoring autophagy (3rd edition). Autophagy, 2016. 12(1): p. 1-222.

309. Meulendyke, K.A., J.D. Croteau, and M.C. Zink, HIV life cycle, innate immunity and autophagy in the central nervous system. Curr Opin HIV AIDS, 2014. 9(6): p. 56571.

310. Cao, L., et al., Methamphetamine potentiates HIV-1 gp120-mediated autophagy via Beclin-1 and Atg5/7 as a pro-survival response in astrocytes. Cell Death Dis, 2016. 7(10): p. e2425.

311. Bagasra, O., et al., Cellular reservoirs of HIV-1 in the central nervous system of infected individuals: identification by the combination of in situ polymerase chain reaction and immunohistochemistry. AIDS, 1996. 10(6): p. 573-85.

312. Nath, A., Eradication of human immunodeficiency virus from brain reservoirs. J Neurovirol, 2015. 21(3): p. 227-34.

313. Sabatier, J.M., et al., Evidence for neurotoxic activity of tat from human immunodeficiency virus type 1. J Virol, 1991. 65(2): p. 961-7.

314. Nath, A., et al., Synergistic neurotoxicity by human immunodeficiency virus proteins Tat and gp120: protection by memantine. Ann Neurol, 2000 . 47(2): p. 18694.

315. Longordo, F., et al., The human immunodeficiency virus-1 protein transactivator of transcription up-regulates $N$-methyl-D-aspartate receptor function by acting at metabotropic glutamate receptor 1 receptors coexisting on human and rat brain noradrenergic neurones. J Pharmacol Exp Ther, 2006. 317(3): p. 1097-105.

316. Fitting, S., et al., Interactive HIV-1 Tat and morphine-induced synaptodendritic injury is triggered through focal disruptions in $\mathrm{Na}(+)$ influx, mitochondrial instability, and $\mathrm{Ca}(2)(+)$ overload. J Neurosci, 2014. 34(38): p. 12850-64.

317. Hauser, K.F., et al., mu-Opioid receptor-induced Ca2+ mobilization and astroglial development: morphine inhibits DNA synthesis and stimulates cellular hypertrophy through a Ca(2+)-dependent mechanism. Brain Res, 1996. 720(1-2): p. 191-203.

318. Hasbani, M.J., et al., Distinct Roles for Sodium, Chloride, and Calcium in Excitotoxic Dendritic Injury and Recovery. Experimental Neurology, 1998. 154: p. 241-258.

319. Lipton, S.A., AIDS-related dementia and calcium homeostasis. Ann N Y Acad Sci, 1994. 747: p. 205-24.

320. Greenwood, S.M., et al., Mitochondrial Dysfunction and Dendritic Beading during Neuronal Toxicity. Journal of Biological Chemistry, 2007. 282: p. 26235-26244.

321. Ellis, R., D. Langford, and E. Masliah, HIV and antiretroviral therapy in the brain: neuronal injury and repair. Nat Rev Neurosci, 2007. 8(1): p. 33-44. 
322. Frake, R.A., et al., Autophagy and neurodegeneration. J Clin Invest, 2015. 125(1): p. 65-74.

323. Menzies, F.M., A. Fleming, and D.C. Rubinsztein, Compromised autophagy and neurodegenerative diseases. Nat Rev Neurosci, 2015. 16(6): p. 345-57.

324. Fitting, S., et al., Opiate addiction therapies and HIV-1 Tat: interactive effects on glial $[\mathrm{Ca}(2)(+)]$, oxyradical and neuroinflammatory chemokine production and correlative neurotoxicity. Curr HIV Res, 2014. 12(6): p. 424-34.

325. Zhao, T., et al., Silencing the PTEN gene is protective against neuronal death induced by human immunodeficiency virus type 1 Tat. J Neurovirol, 2007. 13(2): p. 97-106.

326. Liao, D., et al., Distinct effects of individual opioids on the morphology of spines depend upon the internalization of mu opioid receptors. Mol Cell Neurosci, 2007. 35(3): p. 456-69.

327. Nath, A., Human immunodeficiency virus-associated neurocognitive disorder: pathophysiology in relation to drug addiction. Ann N Y Acad Sci, 2010. 1187: p. 122-8.

328. Kaul, M., et al., HIV-1 coreceptors CCR5 and CXCR4 both mediate neuronal cell death but CCR5 paradoxically can also contribute to protection. Cell Death Differ, 2007. 14(2): p. 296-305.

329. Johnson, E.M. and M.H. Tuszynski, 4 - NEUROTROPHIC FACTORS, in CNS Regeneration (Second Edition), J.H. Kordower and M.H. Tuszynski, Editors. 2008, Academic Press: San Diego. p. 95-144.

330. Hefti, F., B. Knusel, and P.A. Lapchak, Protective effects of nerve growth factor and brain-derived neurotrophic factor on basal forebrain cholinergic neurons in adult rats with partial fimbrial transections. Prog Brain Res, 1993. 98: p. 257-63.

331. Szebenyi, G., et al., Fibroblast growth factor-2 promotes axon branching of cortical neurons by influencing morphology and behavior of the primary growth cone. J Neurosci, 2001. 21(11): p. 3932-41.

332. Katsuki, H., Y. Itsukaichi, and N. Matsuki, Distinct signaling pathways involved in multiple effects of basic fibroblast growth factor on cultured rat hippocampal neurons. Brain Res, 2000. 885(2): p. 240-50.

333. Hu, P., et al., Loss of survival factors and activation of inflammatory cascades in brain sympathetic centers in type 1 diabetic mice. Am J Physiol Endocrinol Metab, 2015. 308(8): p. E688-98.

334. Ozdinler, P.H. and J.D. Macklis, IGF-I specifically enhances axon outgrowth of corticospinal motor neurons. Nat Neurosci, 2006. 9(11): p. 1371-81. 
335. Corvin, A.P., et al., Insulin-like growth factor 1 (IGF1) and its active peptide (13)IGF1 enhance the expression of synaptic markers in neuronal circuits through different cellular mechanisms. Neurosci Lett, 2012. 520(1): p. 51-6.

336. Imai, Y. and S. Kohsaka, Intracellular signaling in M-CSF-induced microglia activation: role of Iba1. Glia, 2002. 40(2): p. 164-74.

337. Hamilton, J.A., Colony-stimulating factors in inflammation and autoimmunity. Nat Rev Immunol, 2008. 8(7): p. 533-44.

338. Luo, J., et al., Colony-stimulating factor 1 receptor (CSF1R) signaling in injured neurons facilitates protection and survival. J Exp Med, 2013. 210(1): p. 157-72.

339. Malik, S., et al., A growth factor attenuates HIV-1 Tat and morphine induced damage to human neurons: implication in HIV/AIDS-drug abuse cases. PLoS One, 2011. 6(3): p. e18116.

340. Nixon, R.A. and A.M. Cataldo, The endosomal-lysosomal system of neurons: new roles. Trends Neurosci, 1995. 18(11): p. 489-96.

341. Liu, Y., et al., Uptake of HIV-1 tat protein mediated by low-density lipoprotein receptor-related protein disrupts the neuronal metabolic balance of the receptor ligands. Nat Med, 2000. 6(12): p. 1380-7.

342. Xiao, H., et al., Selective CXCR4 antagonism by Tat: implications for in vivo expansion of coreceptor use by HIV-1. Proc Natl Acad Sci U S A, 2000. 97(21): p. 11466-71.

343. Tyagi, M., et al., Internalization of HIV-1 tat requires cell surface heparan sulfate proteoglycans. J Biol Chem, 2001. 276(5): p. 3254-61.

344. Marks, A.R., Intracellular calcium-release channels: regulators of cell life and death. Am J Physiol, 1997. 272(2 Pt 2): p. H597-605.

345. Magnuson, D.S., et al., Human immunodeficiency virus type 1 tat activates non- $N$ methyl-D-aspartate excitatory amino acid receptors and causes neurotoxicity. Ann Neurol, 1995. 37(3): p. 373-80.

346. Bigford, G.E., et al., A novel protein complex in membrane rafts linking the NR2B glutamate receptor and autophagy is disrupted following traumatic brain injury. $\mathrm{J}$ Neurotrauma, 2009. 26(5): p. 703-20.

347. Perez-Carrion, M.D., et al., Dendrimer-mediated siRNA delivery knocks down Beclin 1 and potentiates NMDA-mediated toxicity in rat cortical neurons. J Neurochem, 2012. 120(2): p. 259-68.

348. Fields, J.A., et al., Mechanisms of HIV-1 Tat neurotoxicity via CDK5 translocation and hyper-activation: role in HIV-associated neurocognitive disorders. Curr HIV Res, 2015. 13(1): p. 43-54. 
349. Song, D.D., et al., Sphingosine kinase 2 activates autophagy and protects neurons against ischemic injury through interaction with Bcl-2 via its putative BH3 domain. Cell Death Dis, 2017. 8(7): p. e2912.

350. Yang, Y., et al., RNF146 Inhibits Excessive Autophagy by Modulating the Wntbeta-Catenin Pathway in Glutamate Excitotoxicity Injury. Front Cell Neurosci, 2017. 11: p. 59.

351. Wang, Y., et al., p53 induction contributes to excitotoxic neuronal death in rat striatum through apoptotic and autophagic mechanisms. Eur J Neurosci, 2009. 30(12): p. 2258-70.

352. Bossy-Wetzel, E. and D.R. Green, Assays for cytochrome c release from mitochondria during apoptosis. Methods Enzymol, 2000. 322: p. 235-42.

353. Mauvezin, C., et al., Autophagosome-lysosome fusion is independent of VATPase-mediated acidification. Nat Commun, 2015. 6: p. 7007.

354. Yu, X.M. and M.W. Salter, Gain control of NMDA-receptor currents by intracellular sodium. Nature, 1998. 396(6710): p. 469-74.

355. Yu, X.M., The Role of Intracellular Sodium in the Regulation of NMDA-ReceptorMediated Channel Activity and Toxicity. Mol Neurobiol, 2006. 33(1): p. 63-80.

356. Vander Jagt, T.A., J.A. Connor, and C.W. Shuttleworth, Localized loss of Ca2+ homeostasis in neuronal dendrites is a downstream consequence of metabolic compromise during extended NMDA exposures. J Neurosci, 2008. 28(19): p. 5029-39.

357. Xin, W.K., et al., A functional interaction of sodium and calcium in the regulation of NMDA receptor activity by remote NMDA receptors. J Neurosci, 2005. 25(1): p. $139-48$.

358. Greenwood, S.M. and C.N. Connolly, Dendritic and mitochondrial changes during glutamate excitotoxicity. Neuropharmacology, 2007. 53(8): p. 891-8.

359. Liu, Y., et al., Autosis is a Na+,K+-ATPase-regulated form of cell death triggered by autophagy-inducing peptides, starvation, and hypoxia-ischemia. Proc Natl Acad Sci U S A, 2013. 110(51): p. 20364-71.

360. Zhang, G., et al., Induction of a Na(+)/K(+)-ATPase-dependent form of autophagy triggers preferential cell death of human immunodeficiency virus type-1-infected macrophages. Autophagy, 2018. 14(8): p. 1359-1375.

361. Ikegaya, Y., et al., Rapid and reversible changes in dendrite morphology and synaptic efficacy following NMDA receptor activation: implication for a cellular defense against excitotoxicity. J Cell Sci, 2001. 114(Pt 22): p. 4083-93.

362. Tsujimoto, Y. and S. Shimizu, Another way to die: autophagic programmed cell death. Cell Death Differ, 2005. 12 Suppl 2: p. 1528-34. 
363. Liu, Y. and B. Levine, Autosis and autophagic cell death: the dark side of autophagy. Cell Death Differ, 2015. 22(3): p. 367-76.

364. Pickford, F., et al., The autophagy-related protein beclin 1 shows reduced expression in early Alzheimer disease and regulates amyloid beta accumulation in mice. J Clin Invest, 2008. 118(6): p. 2190-9.

365. Spencer, B., et al., Beclin 1 gene transfer activates autophagy and ameliorates the neurodegenerative pathology in alpha-synuclein models of Parkinson's and Lewy body diseases. J Neurosci, 2009. 29(43): p. 13578-88.

366. Ellis, R., D. Langford, and E. Masliah, HIV and antiretroviral therapy in the brain: neuronal injury and repair. Nature Reviews Neuroscience, 2007. 8: p. 33-44.

367. Boven, L.A., et al., Overexpression of nerve growth factor and basic fibroblast growth factor in AIDS dementia complex. J Neuroimmunol, 1999. 97(1-2): p. 15462.

368. Isackson, P.J., Trophic factor response to neuronal stimuli or injury. Curr Opin Neurobiol, 1995. 5(3): p. 350-7.

369. Mark, R.J., et al., Basic FGF attenuates amyloid beta-peptide-induced oxidative stress, mitochondrial dysfunction, and impairment of $\mathrm{Na+} / K_{+}-A T P a s e$ activity in hippocampal neurons. Brain Res, 1997. 756(1-2): p. 205-14.

370. Pechan, P.A., et al., Glutamate induces the growth factors NGF, bFGF, the receptor FGF-R1 and c-fos $m R N A$ expression in rat astrocyte culture. Neurosci Lett, 1993. 153(1): p. 111-4.

371. Hefti, F., Neurotrophic factor therapy for nervous system degenerative diseases. J Neurobiol, 1994. 25(11): p. 1418-35.

372. Nomenclature and research case definitions for neurologic manifestations of human immunodeficiency virus-type 1 (HIV-1) infection. Neurology, 1991. 41(6): p. $778-778$.

373. Antinori, A., et al., Updated research nosology for HIV-associated neurocognitive disorders. Neurology, 2007. 69(18): p. 1789-99.

374. Clifford, D.B. and B.M. Ances, HIV-associated neurocognitive disorder. Lancet Infect Dis, 2013. 13(11): p. 976-86.

375. Heaton, R.K., et al., HIV-associated neurocognitive disorders before and during the era of combination antiretroviral therapy: differences in rates, nature, and predictors. J Neurovirol, 2011. 17(1): p. 3-16.

376. Woods, S.P., et al., Cognitive neuropsychology of HIV-associated neurocognitive disorders. Neuropsychol Rev, 2009. 19(2): p. 152-68. 
377. Heaton, R.K., et al., HIV-associated neurocognitive disorders persist in the era of potent antiretroviral therapy: CHARTER Study. Neurology, 2010. 75(23): p. 208796.

378. Wiley, C.A., et al., Distribution of brain HIV load in AIDS. Brain Pathol, 1998. 8(2): p. 277-84.

379. Moore, D.J., et al., Cortical and subcortical neurodegeneration is associated with HIV neurocognitive impairment. AIDS, 2006. 20(6): p. 879-87.

380. Berger, J.R. and A. Nath, HIV dementia and the basal ganglia. Intervirology, 1997. 40(2-3): p. 122-31.

381. Aylward, E.H., et al., Reduced basal ganglia volume in HIV-1-associated dementia: results from quantitative neuroimaging. Neurology, 1993. 43(10): p. 2099-104.

382. Albright, A.V., S.S. Soldan, and F. Gonzalez-Scarano, Pathogenesis of human immunodeficiency virus-induced neurological disease. J Neurovirol, 2003. 9(2): p. 222-7.

383. Wang, H., K.N. Gracy, and V.M. Pickel, Mu-opioid and NMDA-type glutamate receptors are often colocalized in spiny neurons within patches of the caudateputamen nucleus. J Comp Neurol, 1999. 412(1): p. 132-46.

384. Mirsattari, S.M., C. Power, and A. Nath, Parkinsonism with HIV infection. Mov Disord, 1998. 13(4): p. 684-9.

385. Berger, J.R. and G. Arendt, HIV dementia: the role of the basal ganglia and dopaminergic systems. J Psychopharmacol, 2000. 14(3): p. 214-21.

386. Tse, W., et al., Movement disorders and AIDS: a review. Parkinsonism Relat Disord, 2004. 10(6): p. 323-34.

387. Karlsen, N.R., I. Reinvang, and S.S. Froland, Slowed reaction time in asymptomatic HIV-positive patients. Acta Neurol Scand, 1992. 86(3): p. 242-6.

388. Ogunrin, O. and F. Odiase, Motor speed and reaction time in HIV/AIDS patients: a case-control study. Afr J AIDS Res, 2006. 5(3): p. 217-20.

389. Sweeney, J.A., et al., Pursuit eye movement dysfunction in HIV-1 seropositive individuals. J Psychiatry Neurosci, 1991. 16(5): p. 247-52.

390. Valcour, V., et al., Aging exacerbates extrapyramidal motor signs in the era of highly active antiretroviral therapy. J Neurovirol, 2008. 14(5): p. 362-7.

391. Smith, J.P., et al., Quantitative measurement of muscle strength in the mouse. J Neurosci Methods, 1995. 62(1-2): p. 15-9.

392. Paxinos, G., Paxinos and Franklin's the mouse brain in stereotaxic coordinates, K.B.J. Franklin, Editor. 2013, Boston :: Amsterdam :. 
393. Garcia-Cabezas, M.A., et al., Distinction of Neurons, Glia and Endothelial Cells in the Cerebral Cortex: An Algorithm Based on Cytological Features. Front Neuroanat, 2016. 10: p. 107.

394. Sherwood, C.C., et al., Evolution of increased glia-neuron ratios in the human frontal cortex. Proc Natl Acad Sci U S A, 2006. 103(37): p. 13606-11.

395. Pelvig, D.P., et al., Neocortical glial cell numbers in human brains. Neurobiol Aging, 2008. 29(11): p. 1754-62.

396. Hahn, Y.K., et al., Central HIV-1 Tat exposure elevates anxiety and fear conditioned responses of male mice concurrent with altered mu-opioid receptormediated G-protein activation and beta-arrestin 2 activity in the forebrain. Neurobiol Dis, 2016. 92(Pt B): p. 124-36.

397. Goodkin, K., et al., Subtle neuropsychological impairment and minor cognitivemotor disorder in HIV-1 infection. Neuroradiological, neurophysiological, neuroimmunological, and virological correlates. Neuroimaging Clin N Am, 1997. 7(3): p. 561-79.

398. Simioni, S., et al., Cognitive dysfunction in HIV patients despite long-standing suppression of viremia. AIDS, 2010. 24(9): p. 1243-50.

399. Gonek, M., et al., CCR5 mediates HIV-1 Tat-induced neuroinflammation and influences morphine tolerance, dependence, and reward. Brain Behav Immun, 2018. 69: p. 124-138.

400. Gold, L.H., et al., Prolonged tolerance, dependence and abstinence following subcutaneous morphine pellet implantation in the rat. Eur J Pharmacol, 1994. 253(1-2): p. 45-51.

401. Belknap, J.K., Components of the opioid withdrawal syndrome in mice are thermoregulatory responses. Pharmacol Biochem Behav, 1989. 34(2): p. 241-5.

402. McLane, V.D., et al., Long-term morphine delivery via slow release morphine pellets or osmotic pumps: Plasma concentration, analgesia, and naloxoneprecipitated withdrawal. Life Sci, 2017. 185: p. 1-7.

403. Weiss, J., M.L. Thompson, and L. Shuster, Effects of naloxone and naltrexone on drug-induced hypothermia in mice. Neuropharmacology, 1984. 23(5): p. 483-9.

404. Brooks, S.P. and S.B. Dunnett, Tests to assess motor phenotype in mice: a user's guide. Nat Rev Neurosci, 2009. 10(7): p. 519-29.

405. Jones, B.J. and D.J. Roberts, The quantiative measurement of motor incoordination in naive mice using an acelerating rotarod. J Pharm Pharmacol, 1968. 20(4): p. 302-4. 
406. Nevins, M.E., S.A. Nash, and P.M. Beardsley, Quantitative grip strength assessment as a means of evaluating muscle relaxation in mice. Psychopharmacology (Berl), 1993. 110(1-2): p. 92-6.

407. Fitting, S., et al., Dose-dependent long-term effects of Tat in the rat hippocampal formation: a design-based stereological study. Hippocampus, 2010. 20(4): p. 46980.

408. Maragos, W.F., et al., Neuronal injury in hippocampus with human immunodeficiency virus transactivating protein, Tat. Neuroscience, 2003. 117(1): p. $43-53$.

409. Bruce-Keller, A.J., et al., Synaptic transport of human immunodeficiency virus-Tat protein causes neurotoxicity and gliosis in rat brain. J Neurosci, 2003. 23(23): p. 8417-22.

410. Berrios, I., C. Castro, and D.P. Kuffler, Morphine: axon regeneration, neuroprotection, neurotoxicity, tolerance, and neuropathic pain. P R Health Sci J, 2008. 27(2): p. 119-28.

411. Wang, B., et al., The Neuroprotection of Low-Dose Morphine in Cellular and Animal Models of Parkinson's Disease Through Ameliorating Endoplasmic Reticulum (ER) Stress and Activating Autophagy. Front Mol Neurosci, 2018. 11: p. 120.

412. Lanciego, J.L., N. Luquin, and J.A. Obeso, Functional neuroanatomy of the basal ganglia. Cold Spring Harb Perspect Med, 2012. 2(12): p. a009621.

413. Saylor, D., et al., HIV-associated neurocognitive disorder--pathogenesis and prospects for treatment. Nat Rev Neurol, 2016. 12(4): p. 234-48.

414. Reid, W.C., et al., Neurobehavioral Abnormalities in the HIV-1 Transgenic Rat Do Not Correspond to Neuronal Hypometabolism on 18F-FDG-PET. PLoS One, 2016. 11(3): p. e0152265.

415. June, H.L., et al., Vitamin A deficiency and behavioral and motor deficits in the human immunodeficiency virus type 1 transgenic rat. J Neurovirol, 2009. 15(5-6): p. 380-9.

416. Liu, Z., et al., HIV transactivator of transcription enhances methamphetamineinduced Parkinson's-like behavior in the rats. Neuroreport, 2014. 25(11): p. 860864.

417. Hahn, Y.K., et al., Effects of chronic HIV-1 Tat exposure in the CNS: heightened vulnerability of males versus females to changes in cell numbers, synaptic integrity, and behavior. Brain Struct Funct, 2015. 220(2): p. 605-23.

418. Royal, W., 3rd, et al., Cigarette smoke and nicotine effects on brain proinflammatory responses and behavioral and motor function in HIV-1 transgenic rats. J Neurovirol, 2018. 24(2): p. 246-253. 
419. Schier, C.J., et al., Selective Vulnerability of Striatal D2 versus D1 Dopamine Receptor-Expressing Medium Spiny Neurons in HIV-1 Tat Transgenic Male Mice. J Neurosci, 2017. 37(23): p. 5758-5769.

420. Paris, J.J., et al., 5alpha-reduced progestogens ameliorate mood-related behavioral pathology, neurotoxicity, and microgliosis associated with exposure to HIV-1 Tat. Brain Behav Immun, 2016. 55: p. 202-214.

421. Desfosses, Y., et al., Regulation of human immunodeficiency virus type 1 gene expression by clade-specific Tat proteins. J Virol, 2005. 79(14): p. 9180-91.

422. Wang, T., et al., HIV Tat protein affects circadian rhythmicity by interfering with the circadian system. HIV Med, 2014. 15(9): p. 565-70.

423. Johnson, T.P., et al., Induction of IL-17 and nonclassical T-cell activation by HIVTat protein. Proc Natl Acad Sci U S A, 2013. 110(33): p. 13588-93.

424. Gonzalez-Cano, R., et al., Effects of Tetrodotoxin in Mouse Models of Visceral Pain. Mar Drugs, 2017. 15(6).

425. Luccarini, P., et al., Synergistic antinociceptive effect of amitriptyline and morphine in the rat orofacial formalin test. Anesthesiology, 2004. 100(3): p. 690-6.

426. Stone, L.S., et al., Morphine and clonidine combination therapy improves therapeutic window in mice: synergy in antinociceptive but not in sedative or cardiovascular effects. PLoS One, 2014. 9(10): p. e109903.

427. Lilius, T., et al., Ketamine and norketamine attenuate oxycodone tolerance markedly less than that of morphine: from behaviour to drug availability. $\mathrm{Br} \mathrm{J}$ Anaesth, 2018. 120(4): p. 818-826.

428. Kazantzis, N.P., et al., Opioid and cannabinoid synergy in a mouse neuropathic pain model. Br J Pharmacol, 2016. 173(16): p. 2521-31.

429. Cartmell, S.M., L. Gelgor, and D. Mitchell, A revised rotarod procedure for measuring the effect of antinociceptive drugs on motor function in the rat. $\mathrm{J}$ Pharmacol Methods, 1991. 26(2): p. 149-59.

430. Prodoehl, J., D.M. Corcos, and D.E. Vaillancourt, Basal ganglia mechanisms underlying precision grip force control. Neurosci Biobehav Rev, 2009. 33(6): p. 900-8.

431. Maurissen, J.P., et al., Factors affecting grip strength testing. Neurotoxicol Teratol, 2003. 25(5): p. 543-53.

432. Marcario, J.K., et al., Effects of Morphine on Behavioral Task Performance in SIVInfected Rhesus Macaques. J Neuroimmune Pharmacol, 2016. 11(2): p. 348-57.

433. Deacon, R.M., Measuring motor coordination in mice. J Vis Exp, 2013(75): p. e2609. 
434. Lalonde, R. and C. Strazielle, Brain regions and genes affecting postural control. Prog Neurobiol, 2007. 81(1): p. 45-60.

435. Kaul, M., et al., HIV-1 infection and AIDS: consequences for the central nervous system. Cell Death Differ, 2005. 12 Suppl 1: p. 878-92.

436. Hahn, Y.K., et al., Chronic HIV-1 Tat and HIV reduce Rbfox3/NeuN: evidence for sex-related effects. Curr HIV Res, 2015. 13(1): p. 10-20.

437. Zauli, G., et al., HIV-1 Tat-mediated inhibition of the tyrosine hydroxylase gene expression in dopaminergic neuronal cells. J Biol Chem, 2000. 275(6): p. 4159-65.

438. King, J.E., et al., HIV tat and neurotoxicity. Microbes Infect, 2006. 8(5): p. 134757.

439. Khurdayan, V.K., et al., Preferential vulnerability of astroglia and glial precursors to combined opioid and HIV-1 Tat exposure in vitro. Eur J Neurosci, 2004. 19(12): p. 3171-82.

440. Davis, R.J., Signal transduction by the JNK group of MAP kinases. Cell, 2000. 103(2): p. 239-52.

441. Barber, S.A., et al., Dysregulation of mitogen-activated protein kinase signaling pathways in simian immunodeficiency virus encephalitis. Am J Pathol, 2004. 164(2): p. 355-62.

442. Wei, Y., S. Sinha, and B. Levine, Dual role of JNK1-mediated phosphorylation of $\mathrm{BCl}-2$ in autophagy and apoptosis regulation. Autophagy, 2008. 4(7): p. 949-51.

443. Kaplan, R., et al., A Titrated Morphine Analgesic Regimen Comparing Substance Users and Non-Users with AIDS-Related Pain. Journal of Pain and Symptom Management, 2000. 19: p. 265-273. 
VITA

JESSICA AUDREY LAPIERRE

Born, Hollywood, Florida

2008-2012

B.S., Chemistry

B.A., Classical Studies

University of Florida

Gainesville, Florida

$2012-2014$

M.S., Molecular Pharmacology and Toxicology

University of Southern California

Los Angeles, California

$2014-2018$

Doctoral Candidate

Florida International University

Miami, Florida

\section{PUBLICATIONS AND PRESENTATIONS}

Rodriguez M, Lapierre J, Ojha CR, Pawitwar SS, Muthu Karupan MK, Kashanchi F, ElHage N. (2018) Morphine Counteracts the Antiviral Effect of Antiretroviral Drugs and Causes Upregulation of P62/SQSTM1 and Histone Modifying Enzymes in HIV-Infected Astrocytes. NeuroVirology, 2018, Under revision.

Ojha CR, Rodriguez M, Lapierre J, Muthu Karupan MK, Kashanchi F, El-Hage N. (2018) Toll-like Receptor 3 Regulates Zika Virus Infection and Associated Host Inflammatory Response in Primary Human Astrocytes. PLoS ONE, 2018, Accepted. PONE-D-18-23819

Ojha CR, Rodriguez M, Lapierre J, Muthu Karupan MK, Branscome H, Kashanchi F, ElHage N. (2018) Complementary Mechanisms Potentially Involved in the Pathology of Zika Virus. Front. Immunol., October 2018 https://doi.org/10.3389/fimmu.2018.02340 PubMed PMID: 30374352

Tiwari S, Lapierre J, Ojha CR, Martins K, Parira T, Dutta RK, Caobi A, Garbinsky L, Ceyhan Y, Esteban-Lopez M, El-Hage N (2018) Signaling Pathways and Therapeutic Perspectives Related to Environmental Factors Associated with Multiple Sclerosis. J Neuro Res. 2018; 00:1-16. doi:10.1002/jnr.24322. PubMed PMID: 30204260

Lapierre J, Rodriguez M, Ojha CR, El-Hage N. (2018) Critical Role of Beclin1 in HIV Tat and Morphine-Induced Inflammation and Calcium Release in Glial Cells from Autophagy Deficient Mouse. J Neuroimmune Pharmacol. 2018. doi: 10.1007/s11481-018-9788-3. PubMed PMID: 29752681

Rodriguez M, Lapierre J, Ojha CR, Estrada-Bueno H, Dever SM, Gewirtz DA, Kashanchi F, El-Hage N. (2017) Importance of Autophagy in Mediating Human Immunodeficiency Virus (HIV) and Morphine-Induced Metabolic Dysfunction and Inflammation in Human Astrocytes. Viruses. 2017;9(8):201. doi: 10.3390/v9080201. PubMed PMID: 28788100 
Ojha CR, Lapierre J, Rodriguez M, Dever SM, Zadeh MA, DeMarino C, Pleet ML, Kashanchi F, El-Hage N. (2017) Interplay between Autophagy, Exosomes and HIV-1 Associated Neurological Disorders: New Insights for Diagnosis and Therapeutic Applications. Viruses. 2017 Jul 6;9(7). pii: E176. doi: 10.3390/v9070176. PubMed PMID: 28684681

Rodriguez M, Lapierre J, Ojha CR, Kaushik A, Batrakova E, Kashanchi F, Dever SM, Nair M, El-Hage N (2017). Intranasal drug delivery of small interfering RNA targeting Beclin1 encapsulated with polyethylenimine (PEI) in mouse brain to achieve HIV attenuation. Sci Rep. 2017 May 12;7(1):1862. doi: 10.1038/s41598-017-01819-9. PubMed PMID: 28500326

Pawitwar SS, Dhar S, Tiwari S, Ojha CR, Lapierre J, Martins K, Rodzinski A, Parira T, Paudel I, Li J, Dutta RK, Silva MR, Kaushik A, El-Hage N. (2017) Overview on the Current Status of Zika Virus Pathogenesis and Animal Related Research. J Neuroimmune Pharmacol. 2017 Sep;12(3):371-388. doi: 10.1007/s11481-017-9743-8. PubMed PMID: 28444557

Rodriguez M, Kaushik A, Lapierre J, Dever SM, El-Hage N, Nair M (2016). Electromagnetic nano-particle bound Beclin1 siRNA crosses the blood-brain barrier to attenuate the inflammatory effects of HIV-1 infection in vitro. J Neuroimmune Pharmacol. 2017 Mar;12(1):120-132. doi: 10.1007/s11481-016-9688-3. PubMed PMID: 27287620

Dever SM, Rodriguez M, Lapierre J, Costin B, El-Hage, N (2015). Differing roles of autophagy in HIV-associated neurocognitive impairment and encephalitis with implications for morphine co-exposure. Front. Microbiol. 2015, Jul 6; 6:653. doi: 10.3389/fmicb.2015.00653. PubMed PMID: 26217309

Lapierre J, Rodriguez M, Ojha CR, El-Hage N “Decreased autophagy modulates Tat and morphine-induced inflammatory molecules, growth factors and calcium release in glial cells from hemizygous Becn $1^{+/}$mouse." 2018 Keystone Symposia: Selective Autophagy, Kyoto, Japan, April $22^{\text {nd }}-26^{\text {th }} 2018$

Lapierre J, Rodriquez M, Ojha CR, Estrada-Bueno H, El-Hage N. "Molecular characterization of Beclin1 association with HIV-Tat and opiate-induced inflammation using glial cells from autophagy-deficient mice." 2017 NIDA Diversity Supplement Trainee Workshop, Bethesda MD, May $3^{\text {rd }}-5^{\text {th }} 2017$

Lapierre J, Rodriguez M, El-Hage N "Autophagy is cytoprotective in neurons and necessary against tat and morphine-induced toxicity in autophagy-deficient mice." 23rd Scientific Conference of the Society on Neuroimmune Pharmacology, Philadelphia, PA, March $29^{\text {th }}-$ April $1^{\text {st }} 2017$

Lapierre J, Rodriquez M, El-Hage N. "Role of autophagy in HIV-1 Tat induced striatal motor neurodegeneration in combination with morphine co-exposure using heterozygous Beclin-1 mouse model." 22nd Scientific Conference of the Society on Neuroimmune Pharmacology, Krakow, Poland, April $5^{\text {th }}-9^{\text {th }} 2016$ 